



\section{Equipment for printing of \\ high viscosity liquids and molten metals}

René Houben 


\section{Samenstelling promotiecommissie:}

Prof. dr. G. (Gerard) van der Steenhoven (voorzitter) Universiteit Twente Prof. dr. ir. J.F. (Frits) Dijksman (promotor) Universiteit Twente

Prof. dr. rer. nat. D. (Detlef) Lohse (promotor) Universiteit Twente Prof. dr. ir. A.J. (Bert) Huis in 't Veld Universiteit Twente

Prof. dr. ir. J. (Han) Huétink Universiteit Twente

Dr. ir. Y.H. (Ysbrand) Wijnant Universiteit Twente Prof. dr. ir. A.A. (Anton) van Steenhoven Universiteit Eindhoven Prof. dr. A. (Andreas) Schmidt-Ott Universiteit Delft

The work in this thesis was carried out at the Equipment for Additive Manufacturing department of TNO in cooperation with the Physics of Fluids group of the Faculty of Science and Technology and of Mesa+ of the University of Twente.

Nederlandse titel:

Apparatuur voor het printen van visceuze vloeistoffen en vloeibare metalen

Publisher:

René Houben, Equipment for Additive Manufacturing, TNO, P.O. Box 6235, 5600 HE Eindhoven, The Netherlands www.tno.nl/rm

(c) René Houben, Eindhoven, The Netherlands, 2012

No part of this work may be reproduced by print photocopy or any other means without the permission in writing from the publisher ISBN: 978-90-365-3404-8 


\section{EQUIPMENT FOR PRINTING OF HIGH VISCOSITY LIQUIDS AND MOLTEN METALS}

\section{PROEFSCHRIFT}

ter verkrijging van

de graad van doctor aan de Universiteit Twente,

op gezag van de rector magnificus,

Prof. dr. H. Brinksma,

volgens besluit van het College voor Promoties

in het openbaar te verdedigen

op donderdag 27 september 2012 om 14.45 uur

door

René Jos Houben

geboren op 26 mei 1978

te Geldrop 
Dit proefschrift is goedgekeurd door de promotoren:

Prof. dr. ir. J.F. (Frits) Dijksman

Prof. dr. rer. nat. D. (Detlef) Lohse 


\section{Contents}

1 Introduction 1

1.1 Additive manufacturing . . . . . . . . . . . . . 2

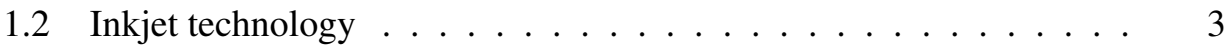

1.3 Outline of this thesis $\ldots \ldots \ldots \ldots \ldots$

2 Advances in high viscosity jetting 9

2.1 Printhead development . . . . . . . . . . . . . . . 10

2.1.1 General system selection and concept design . . . . . . . 10

2.1.2 Theoretical achievability of high viscosity jetting . . . . . 23

2.1 .3 Final design . . . . . . . . . . . . . . . 33

2.2 Material supply system . . . . . . . . . . . . . . . . . . 34

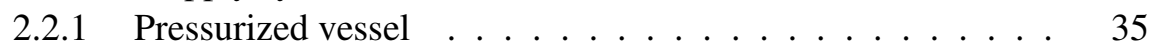

2.2.2 Constant flow dampened system . . . . . . . . . . . . 36

2.3 Droplet visualisation . . . . . . . . . . . . . . 38

2.3.1 Stroboscopic illumination combined with a free running or triggered camera . . . . . . . . . . . . 38

2.3.2 Static illumination combined with a high speed camera . . . 39

2.4 Experimental validation . . . . . . . . . . . . . . . . 40

2.5 Discussion . . . . . . . . . . . . . . . . . 42

2.6 Alternative designs . . . . . . . . . . . . . . . . 43

2.6 .1 Vibrating nozzle . . . . . . . . . . . . . . . 44

2.6.2 Mechanical droplet break-up . . . . . . . . . . . . . . . . 4 44

2.6.3 Pressure independent droplet generation . . . . . . . . . . . 46

2.7 Concluding remarks . . . . . . . . . . . . . . . . . 47

3 Droplet selection $\quad 49$

3.1 From electrostatic deflection towards alternatives . . . . . . . . 50

3.1.1 Selection of alternative droplet selection methods . . . . . . 52

3.2 Ballistic deflection, droplet selection by collision . . . . . . . . 54

3.2 .1 Experimental setup . . . . . . . . . . . . . . . 60 
3.2.2 Experimental results . . . . . . . . . . . . . 61

3.2 .3 Discussion . . . . . . . . . . . . . . . . 63

3.3 Droplet selection by air-jet . . . . . . . . . . . . 65

3.3.1 Performance of small stationary air-jets . . . . . . . . 67

3.3 .2 Moving the air-jet . . . . . . . . . . . . 72

3.3.3 Combination of the air-jet deflection mechanism with HVJ . $\quad 74$

3.3 .4 Discussion . . . . . . . . . . . . . . 75

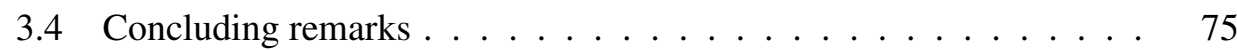

4 Other applications of high viscosity jetting; spray drying \& droplet encapsulation $\quad \mathbf{7 7}$

4.1 From droplet generation to monodisperse powders . . . . . . . . . 78

4.1 .1 Energy efficiency . . . . . . . . . . . . . . . . 81

4.1 .2 Powder properties . . . . . . . . . . . . . 81

4.1 .3 Discussion ................... . 84

4.2 Inkjet as a means for the making of encapsulates . . . . . . . . 86

$4.2 .1 \quad$ Experimental setup . . . . . . . . . . . . . . . . . 88

4.2 .2 Experimental results $\ldots \ldots \ldots \ldots 8 . \ldots \ldots$

4.2 .3 Discussion . . . . . . . . . . . . . . . . 91

4.2.4 Alternative designs . . . . . . . . . . . . . . . . 92

4.3 Concluding remarks . . . . . . . . . . . . . . . . 95

5 Metal printing $\quad 97$

5.1 Conductive metallic tracks by pyrolytic printing . . . . . . . . 98

5.1.1 Overview of existing metal printing techniques . . . . . . 98

5.1.2 General process description . . . . . . . . . . . . 100

5.1 .3 Experimental setup . . . . . . . . . . . . . . . . . 101

5.1 .4 Experimental results . . . . . . . . . . . . . . . 101

5.1.5 Concluding remarks . . . . . . . . . . . . . 103

5.2 Direct Metal Jetting . . . . . . . . . . . . . . . . . . . . . 103

5.2.1 Overview of metallic rapid manufacturing processes . . . 104

5.2.2 Process description / experimental setup . . . . . . . . . . 107

5.2 .3 Experimental results . . . . . . . . . . . . . . . . . . 108

5.2 .4 Concluding remarks $\ldots \ldots \ldots$. . . . . . . . . . 109

6 3D printing; from layer formation to three dimensional objects $\quad 111$

6.1 Printing functional material . . . . . . . . . . . . . . . 112

6.2 General process description . . . . . . . . . . . . . . . . . . . 112

6.2.1 Droplet generation . . . . . . . . . . . . . . . 114 
6.2.2 Droplet selection . . . . . . . . . . . . . . . . . . 114

6.2 .3 Product build-up . . . . . . . . . . . . . . . . . . 115

6.3 Applications . . . . . . . . . . . . . . . . . 116

6.4 Concluding remarks . . . . . . . . . . . . . . 116

$\begin{array}{lll}7 & \text { Epilogue } & 117\end{array}$

7.1 Additive manufacturing . . . . . . . . . . . . . . . . 118

7.1.1 Which market drivers exist? . . . . . . . . . . . 118

7.1.2 What should be developed to support these drivers? . . . . . 120

7.2 Inkjet development . . . . . . . . . . . . . . . . 121

7.2.1 Disposable nozzle array as a route to industrial reliability . . 122

7.2.2 Integration of chemical processing within the printhead . . . 123

7.3 Concluding remarks . . . . . . . . . . . . . . . . 124

$\begin{array}{ll}\text { A Rapid manufacturing technology overview } & 137\end{array}$

B Overview of available droplet formation methods 147

B.1 Continuous inkjet principles . . . . . . . . . . . . . . . . . 147

B.1.1 Acoustically disrupted fluid jet . . . . . . . . . . . . 147

B.1.2 Hertz mist . . . . . . . . . . . . . . . . . . . . . . . 149

B.1.3 MicroDot . . . . . . . . . . . . . . . . . . . . . 149

B.1.4 Electrohydrodynamic inkjet/ Electrostatic pull inkjet . . . . 150

B.1.5 Jet cutter technology . . . . . . . . . . . . . . . . 151

B.2 Drop-on-demand principles . . . . . . . . . . . . . . . 151

B.2.1 Thermal inkjet . . . . . . . . . . . . . . 152

B.2.2 Thermal spark . . . . . . . . . . . . . . 152

B.2.3 Piezoelectric direct pressure pulse . . . . . . . . . . 153

B.2.4 Focused acoustic beam ejection . . . . . . . . . . . 154

B.2.5 Flex tensional aperture plate inkjet . . . . . . . . . . . 154

B.2.6 Thermal electrostatic inkjet . . . . . . . . . . . . 156

B.2.7 Liquid fault tolerant process . . . . . . . . . . . . 156

B.2.8 Electro-rheological fluid inkjet . . . . . . . . . . . . 157

B.2.9 Thermal-rheological fluid inkjet . . . . . . . . . . 158

B.2.10 Topspot microdrop ejector . . . . . . . . . . . . 158

$\begin{array}{ll}C \text { Boundary curves impact regimes } & 161\end{array}$

$\begin{array}{ll}\text { Summary } & 165\end{array}$

$\begin{array}{ll}\text { Samenvatting } & 171\end{array}$ 
Acknowledgements

About the author 


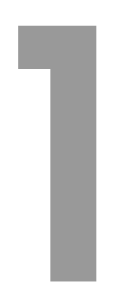

\section{Introduction}

Additive manufacturing is an upcoming manufacturing technology. For years injection moulding has dominated mass manufacturing and layer-wise production technology was only used for prototyping purposes, also known as rapid prototyping. The technology was initiated as a design tool to achieve a quick impression of the final product. Creating $3 D$ models directly from $C A D$ data provided direct feedback to the designers. Most products created with currently available additive manufacturing processes unfortunately can only be used to get an impression from the final product, since the generated product lacks practical applicability. However, if functional end products could be realised with such a process a production method would exist capable of producing customer specific products on demand. If this could be realised for reasonable prices this might lead to a paradigm shift. Keeping stocks would become outdated; shipping products around the world would become unnecessary; on-site production might become profitable, preventing a total production shift to low wage countries. In order to approach such a situation a lot of development is necessary.

The first step can be found in the ability to process the materials necessary to create a final product with the required functional properties. The realised product should at least be as functional as its conventionally manufactured counterpart. The usage of the technology in labour intensive applications leaves room for development, especially where personalized products are required. To evolve to a manufacturing technology the technology needs to break away from these niche markets and bridge the gap from batch towards continuous production layouts, realising competitive produc- 
tion speeds. However the main challenges for rapid prototyping, in order to evolve to a real manufacturing method, remains the availability of deposition systems capable of processing the required materials.

Inkjet printing is very promising in this area. It is one of the few processes that is able to selectively deposit small amounts of material. One can even envision multiple printheads processing different types of material generating a multi material product, or even beyond, namely generating a graded structure; rigid on one side and gradually changing to flexible on the other side; impossible with conventional manufacturing methods. Conventional inkjet systems are very limited with respect to the range of materials they can process, making them virtually unusable for additive manufacturing applications. This thesis describes how the principles of the first inkjet printheads can be reused to achieve printability of high viscous materials, enabling the use of inkjet technology for additive manufacturing purposes.

\subsection{Additive manufacturing}

From the beginning of the 20th century the first ideas were patented about layer wise manufacturing [1,2]. It took until the late 1980's for the technology to become of interest on a larger scale. The first basic principles were developed, from systems based on lasers for curing material, stereolithography (SLA) [3], lasers for selective cutting of material, laminated object manufacturing (LOM) [4], and lasers for sintering of materials, selective laser sintering (SLS) [5]. Or less expensive techniques using extrusion nozzles like fused deposition modelling (FDM) [6].

In this thesis, however, we confine ourselves to principles based on inkjet technology. Two different basic principles can be distinguished. The first, printing objects directly. With each printed layer a cross section of the product is deposited, meaning the final product is build up out of the ink processed by the printhead directly. This technology started in 1987 with the development of ballistic particle manufacturing (BPM) [7], using jetting devices to form a 3D structure. Later Brother Industries described the use of photopolymers for 3D inkjet based systems [8]. These polymers were cured by means of a UV light source resulting in a significant increase of the mechanical properties of the formed products. Followed by patents describing the use of a combination of inkjets using different materials [9], towards the use of multi nozzle printheads for 3D applications [10]. All of these systems share the same drawback; the properties of the end product are solely dependent on the ink jetted.

The second principle uses the ink as a binder, printing on a powder bed, using the ink to glue the powder particles together in an effort to overcome the limitations of the previous system. It took until 1993 before the first powder based inkjet systems 
were developed [11].

Now, approximately 20 years later, these inkjet based systems still struggle with the same issue, namely the limited range of materials processable by means of inkjet printheads. But the unique capability of inkjet to create a multi material or even graded structure has pushed us to develop this technology further. Although there are over 400 other layer-wise production methods developed by now (see appendix A) inkjet is still one of the most promising routes provided the material processing limitations can be overcome.

\subsection{Inkjet technology}

Inkjet is a so called non-contact print technology, meaning ink can be deposited on a substrate without contact between printhead and substrate. By mounting the printhead on a carriage, full 2D or even $3 \mathrm{D}$ control about the landing position of the droplet can be achieved. For graphics and document printing the image is build up out of a matrix of small dots. As mentioned before, one of the main challenges of using inkjet technology for additive manufacturing purposes can be found in whether the technology is able to process a broad range of materials.

A general overview of inkjet technology has been described by Le [12] and more recently by Wijshof [13]. Figure 1.1 shows that inkjet technology can be split up in two main areas; drop-on-demand (dod) inkjet and continuous inkjet (cij). A drop-ondemand print system only generates a droplet when it is needed, where on the other hand a continuous inkjet system is continuously generating droplets. The continuous jet is ejected from the nozzle which breaks-up due to an induced vibration. To allow selective printing a droplet selection mechanism is added to selectively sent the droplets towards the substrate. Commonly an electrostatic deflection mechanism is used, selective charging of the droplets and consequently sending them through an electric field, to control whether the droplets reach the substrate or are recycled into the catcher (figure 1.2).

Both types of systems introduce a pressure perturbation in the ink contained in the printhead. With a drop-on-demand system the perturbation is large enough to eject a droplet out of the nozzle; with a continuous inkjet system the perturbation creates a pressure fluctuation behind the nozzle resulting in controlled jet break-up.

In literature several different droplet formation methods are described $[15,16]$. In appendix B an overview is given of available droplet formation methods. 


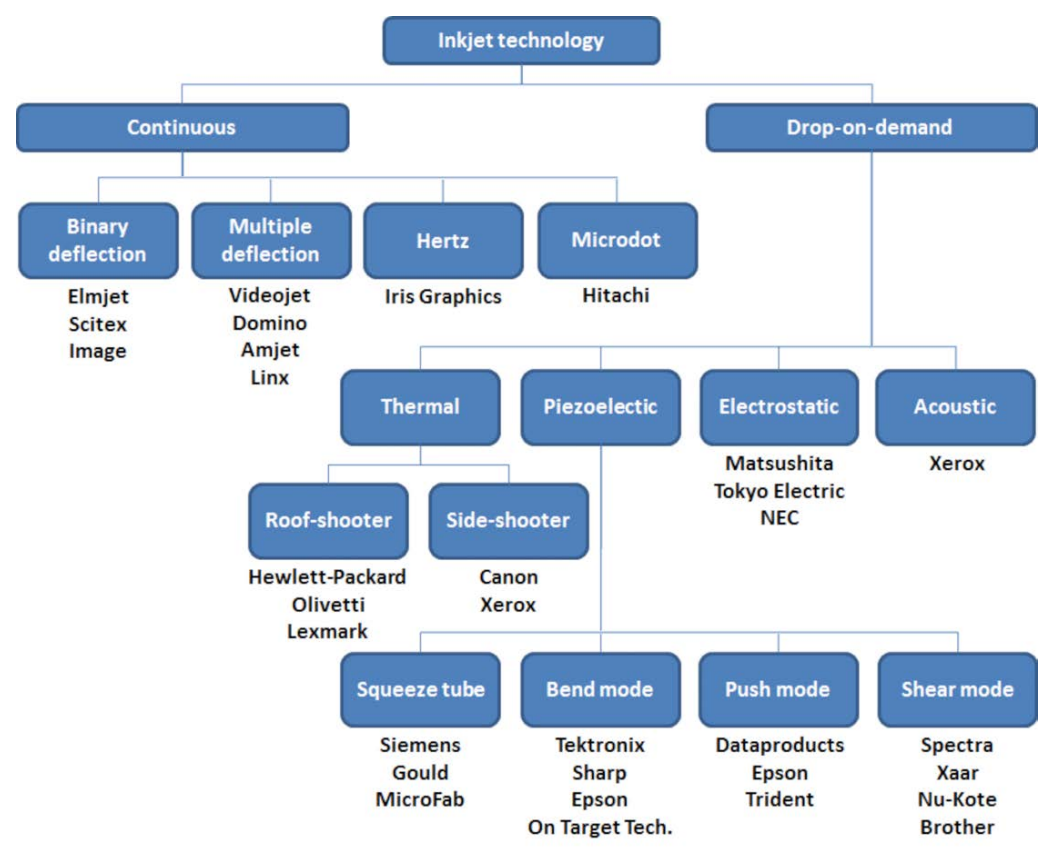

Figure 1.1: Overview inkjet technology [12].

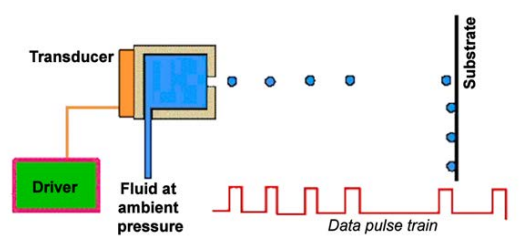

(a) Drop-on-demand.

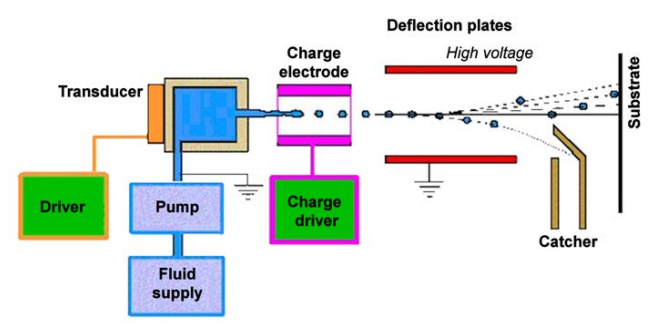

(b) Continuous.

Figure 1.2: Drop-on-demand- and continuous inkjet [14]. 


\subsection{Outline of this thesis}

This thesis reports about the design, realisation and applications of an inkjet technology enabling processing of highly viscous materials (up to 20 times more viscous than with conventional technology) based on published patent applications filed during the development process.

Chapter 2 describes the development of the droplet generation process, starting in 2001 with WO2004018212, "Apparatus and method for printing a fluid material by means of a continuous jet printing technique" [17]. This document describes the basic principle of an inkjet device able to jet high viscosity fluids. The principle used is similar to the concept of the first inkjet printheads. Basically a vibrating element is used to induce stable jet breakup. The patent describes that if the vibration is applied sufficiently close to the nozzle by means of a focusing member, the high intensity pressure fluctuations created cause jet break-up even with high viscous fluids.

The pressures used in the high viscosity inkjet system raise well above several hundreds of bars. Therefore in WO2008060149 "Constant flow high pressure printing system" [18] a material supply system is described delivering a material flow without any perturbations in the required high pressure regimes. The inkjet principle uses pressure fluctuations to create stable droplets break-up of the fluid jet. It is therefore preferred to use a fluid supply system delivering the material without any fluctuations to prevent interference of fluctuations from the fluid supply system with the jet breakup. Contrary to normally used pressure regulated inkjet systems the described system uses a controlled flow to achieve a material supply delivering exact drop size and jet speed independent of viscosity fluctuations, broadening the spectrum of allowable materials even further.

Where WO2004018212 [17] describes the basic principle, in WO2009028947 [19], WO2009061202 [20] and WO2010068108 [21] all titled "Droplet breakup device" alternative actuation principles are described. In WO2009028947 and WO2010068108 an alternative for piezo based actuation is sketched by using a rotating vibration device to deliver the pressure fluctuations near the nozzles, and in WO2009061202 a vibrating mechanism is described equivalent to WO200418212, only in this case the nozzle vibrates and the focusing member close to the nozzle is stationary.

Development of multi nozzle systems brings new design issues, because the high pressures in the system result in high mechanical loads on the piezo element, which limits the performance. Therefore in WO2009151332 "Pressure independent droplet generation" [22] a system is described creating a pressure independent actuation mechanism. The vibrating member is designed pressure neutral, so that raising the 
pressure in the material supply does not result in an extra load on the actuation device, resulting in increased performance with multi nozzle configurations.

Creating droplets of high viscous materials is the first step; selectively depositing the droplets on a specific location on the substrate is the second step, which is described in detail in chapter 3 of this thesis. In conventional continuous inkjet systems electrostatic deflection is used. The droplets are selectively charged and guided by means of an electrostatic field either towards the substrate or in the return gutter. This selection principle is based on the fact that the droplets are chargeable, requiring the material to be slightly conductive. Increasing the viscosity usually results in a lowered conductivity leading to a too low charge on the droplets and consequently a limited possibility for electrostatic deflection. Therefore in EP1869133 "Curable compositions for continuous inkjet printing and methods for using these compositions" [23] a principle is described to achieve higher degrees of conductivity without extensive material adjustments. If no material adjustments are allowable two alternative deflection mechanisms are described in WO2009061201 [24] and WO2009061195 [25] both entitled "Droplet selection mechanism". One document describes the use of a second inkjet to ballistically shoot the undesired droplets out of their path into a recycle gutter, where the other describes a controllable air-jet to alter the droplets path enabling droplet selection.

Although the main focus has been on the development of an inkjet system for application in the field of additive manufacturing, chapter 4 describes how the system has proven to be functional in other application areas as well. The ability to generate monodisperse droplets from high viscous fluids appears to be of great use in powder production applications. WO2008069639 "Method and apparatus for spray drying and powder produced using said method" [26] describes the use of the high viscosity printhead in spray drying applications for the production of powders. The increased control over the droplet generation process leads towards generation of powders with a higher degree of monodispersity. The increased control does not only result in better powder characteristics but also in a higher efficiency of the drying process. Compared to conventional spray nozzles the printhead is capable of processing a higher initial viscosity resulting in a higher energy efficiency of the total process chain.

The control over droplet size is of interest for powder production, the control over the droplet's direction and velocity even enables a droplet encapsulation principle as described in WO2009014432 "Multi component particle generation system" [27]. The droplets generated by the printhead are propelled through a liquid screen resulting in encapsulated droplets exiting the screen. Drying of these encapsulates results 
in multi component particles allowing sensitive materials to be packed inside a protective skin to prevent for instance oxidation. To create very small encapsulates, thin liquid screens are required, WO2010090518 also entitled "Multi component particle generation system" describes a method therefore, by modulating the liquid screen [28]. Analogous to the inkjet based encapsulation principle, WO2010005302 "Multi component particle generating system" [29] describes a system for propelling pregenerated powders through a liquid screen resulting in a method capable of encapsulating powder particles.

Within the field of additive manufacturing, creating functional end products is the first step. Inkjet enables the creation of multi material products, hereby opening the path towards structures with graded material properties. The high viscosity printhead allows a much broader spectrum of materials to be printed making a great leap towards usage of inkjet in additive manufacturing applications. The next step within the field of additive manufacturing is creating 3D products with electronic functionality requiring the deposition of conductive materials. A lot of inkjet research is based on deposition of silver nano materials by means of conventional inkjet systems [30]. Unfortunately, the usage of these types of inks requires post-treatment of the deposited layers to achieve a sufficiently high conductivity. For additive manufacturing processes post treatment steps are not preferred. In chapter 5 alternative paths have been investigated to achieve a high conductivity directly after deposition. WO2009011583 "Method and apparatus for applying a material on a substrate" [31] describes a jetting system creating droplets from a metal salt solution at low temperature which are pyrolised and melted during their flight towards the substrate enabling direct liquid metal deposition using conventional jetting devices. The application describes how the droplets can be focused aerodynamically. Alternatively, one can also envision metals to be printed directly from the melt. This, however, requires extremely high temperatures (up to $1140^{\circ} \mathrm{C}$ ) which are difficult to maintain. Therefore WO2007075084 "Material jet system" [32] describes a system to feed material towards such a printhead allowing the amount of hot molten material to be minimized resulting in a smaller and more energy efficient printhead configuration.

Creation of a printhead which generates droplets is the beginning. Further development leads towards a print system capable of depositioning droplets on a specific position onto a substrate. Chapter 6 describes some of the hurdles which may occur in the process of integrating such a print system in an additive manufacturing machine. Printing enables new possibilities and more freedom for forms and materials where current ways of designing are not sufficient. 
Chapter 7 finalizes the thesis with an epilogue, containing considerations for the future of printing high viscosity materials.

During this thesis the words: inkjet, printing and printhead are frequently used although no conventional "ink" is being processed nor any "documents are printed" with the developed technique, as originally intended with these terms. These words, however, supply the reader with the desired association and are therefore commonly used within the broadening application fields of this technology. 


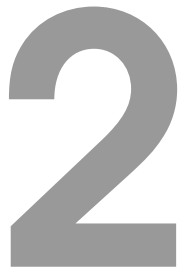 \\ Advances in high viscosity jetting ${ }^{1}$}

The viscosity is a limiting factor in the applicability of conventional inkjet printheads. The higher the viscosity the more difficult it becomes to jet droplets. In this chapter the considerations of the development of a printhead for high viscous fluids are described. From the general system selection towards a more detailed description of the actuation mechanism, the sealing means and the nozzle layout. The words "printhead" refer to the part of a print system meant for the creation of droplets; the most crucial part in the development of a print system. A theoretical process window is sketched together with an analytical model to give an impression of the pressures which can be expected, as an input for the final design. Not only the development of the droplet generating part is described; also different types of material supply

\footnotetext{
${ }^{1}$ The patents on which this chapter is based are filed as:

$\diamond$ R.J. Houben, "Apparatus and method for printing a fluid by means of a continuous jet printing technique", (2004), WO2004018212.

$\diamond$ R.J. Houben, "Constant flow high pressure printing system", (2008), WO2008060149.

$\diamond$ R.J. Houben, L.A.M. Brouwers, A. Rijfers, "Droplet break-up device", (2009), WO2009028947.

$\diamond$ A. Rijfers, R.J. Houben, L.A.M. Brouwers, "Droplet break-up device", (2009), WO2009061202.

$\diamond$ F. de Vreede, A.P. Aulbers, R.J. Houben, "Pressure independent droplet generation", (2009), WO2009151332.

$\diamond$ G.P.H. Gubbels, R.J. Houben, “Droplet based fluid jet polishing”, (2010), WO2010068108.
} 
systems and means for droplet visualisation are discussed. Based on experimental results the performance of the system is assessed and finally alternative embodiments are discussed. The developed system proved to generate droplets effectively, capable of using an initial vibration of only $1.25 \%$ compared to conventional droplet generators to achieve equal drop formation. This more efficient layout made printing of viscous materials a reality.

\subsection{Printhead development}

In the following sub-paragraphs a general system selection procedure will be outlined. Based on a general overview of known droplet generation methods the most appropriate mechanism for generating high viscous droplets for printing applications will be selected. Thereafter several design aspects of the printhead will be discussed followed by a theoretical approximation of the expected performance depending on final dimensioning of the system.

\subsubsection{General system selection and concept design}

The main goal is to develop an inkjet system that can process a broader spectrum of materials compared to current state of art systems. Depending on fluid behaviour several groups can be distinguished, from Newtonian and non-Newtonian fluids towards pseudo plastic fluids, such as suspensions or viscoelastic fluids with a high elongational strength. In this thesis fluids are considered which have Newtonian behaviour, in that case one of the most limiting parameters is the maximum allowable viscosity.

The following requirements are taken as a starting point for the design of the system:

$\diamond$ Ability to process high viscous Newtonian fluids. To mention an example: commonly available UV curable polymers have a viscosity of up to $500 \mathrm{mPa} \cdot \mathrm{s}$. With such materials it will be possible to make 3D structures layer by layer by successive inkjet printing and UV curing, achieving improved mechanical properties of the finished product.

$\diamond$ The actuation mechanism should work independent of the type of fluid (chemical composition independent).

$\diamond$ The actuation mechanism should be able to work at different (high) frequencies to allow frequency tuning and to enable drop size selection using a single nozzle size.

$\diamond$ Simple nozzle design, easily fabricated and replaceable, so multiple sizes can be tested. 
$\diamond$ The amplitude of actuation mechanism should be adjustable, enabling control over droplet formation. Causing jet break-up in a reasonable distance from the nozzle.

$\diamond$ The design should be flexible to allow creation of small droplet sizes to achieve high product accuracies. Low resolution additive manufacturing processes need $1 \mathrm{~mm}$ resolution, very high resolution processes demand for $5 \mu \mathrm{m}$. To maintain production speed drop sizes in the order of $100 \mu \mathrm{m}$ are preferred at this stage.

$\diamond$ Up-scalable to multi nozzle systems to allow high production speeds to be reached.

$\diamond$ The smaller the distance between nozzle and substrate the higher the droplet placement accuracy. Therefore the actuation mechanism should lead to droplet break-up within a reasonable distance from the nozzle, at least within the distance between nozzle plate and substrate.

$\diamond$ Nozzle easily removable/changeable.

$\diamond$ Droplet generator should be flushable.

$\diamond$ Long lifetime.

$\diamond$ Temperature resistant up to $100^{\circ} \mathrm{C}$ to allow viscosity adjustment.

An overview of several actuation methods which have been developed in course of time for the use in inkjet systems is given in appendix B. A summary thereof is given in table 2.1 together with a first shifting keeping in mind whether the principle is suitable for the application described in this thesis.

Table 2.1: First system selection.

\begin{tabular}{|l|l|l|}
\hline $\begin{array}{l}\text { Droplet genera- } \\
\text { tion principle }\end{array}$ & Short description & Applicable \\
\hline $\begin{array}{l}\text { Disrupted con- } \\
\text { tinuous fluid } \\
\text { jet }\end{array}$ & $\begin{array}{l}\text { Continuously generated fluid } \\
\text { jet stimulated to break-up } \\
\text { into droplets in a controlled } \\
\text { manner. }\end{array}$ & Possible. \\
\hline $\begin{array}{l}\text { Herz continuous } \\
\text { mist inkjet }\end{array}$ & $\begin{array}{l}\text { Continuously generated fluid } \\
\text { jet using natural Rayleigh } \\
\text { break-up with extremely } \\
\text { small nozzles. }\end{array}$ & $\begin{array}{l}\text { Using extremely small noz- } \\
\text { zles causes extra challenges } \\
\text { when using higher viscous } \\
\text { fluids. }\end{array}$ \\
\hline \multicolumn{2}{|c|}{ Continued on next page } \\
\hline
\end{tabular}


Table 2.1 - continued from previous page

\begin{tabular}{|c|c|c|}
\hline $\begin{array}{l}\text { Droplet genera- } \\
\text { tion principle }\end{array}$ & Short description & Applicable \\
\hline Microdot & $\begin{array}{l}\text { Similar to disrupted contin- } \\
\text { uous fluid jet, only satel- } \\
\text { lite droplets are used, main } \\
\text { droplets are recycled. }\end{array}$ & Possible. \\
\hline $\begin{array}{l}\text { Electro hydrody- } \\
\text { namic inkjet }\end{array}$ & $\begin{array}{l}\text { Ejecting droplets by means } \\
\text { of pulling them from the noz- } \\
\text { zle by means of a high elec- } \\
\text { trostatic field. }\end{array}$ & $\begin{array}{l}\text { The ink should be conductive } \\
\left(10^{-13} \text { to preferably } 10^{-4}\right. \\
\mathrm{S} / \mathrm{cm}) \text {, and should have a rel- } \\
\text { atively low surface tension, } \\
\text { limiting the scope of print- } \\
\text { able inks. }\end{array}$ \\
\hline $\begin{array}{l}\text { Jet cutter tech- } \\
\text { nology }\end{array}$ & $\begin{array}{l}\text { Continuous fluid jet be- } \\
\text { ing mechanically cut into } \\
\text { droplets. }\end{array}$ & $\begin{array}{l}\text { Direction of droplets is not } \\
\text { well defined, making the } \\
\text { droplet creation principle not } \\
\text { suitable for high precision } \\
\text { printing applications. }\end{array}$ \\
\hline $\begin{array}{l}\text { Thermal inkjet } \\
\text { (bubble jet) }\end{array}$ & $\begin{array}{l}\text { Droplets are propelled out of } \\
\text { a nozzle by means of a shock- } \\
\text { wave caused by fast heating } \\
\text { and consequently boiling of } \\
\text { the ink. }\end{array}$ & $\begin{array}{l}\text { Bubble creation is mostly de- } \\
\text { signed for water based inks. } \\
\text { It can however be redesigned } \\
\text { to work with other solvents. } \\
\text { However, the ink should be } \\
\text { designed to withstand these } \\
\text { temperatures. To maintain a } \\
\text { broad material spectrum, se- } \\
\text { lective heating is not desired. }\end{array}$ \\
\hline Thermal-Spark & $\begin{array}{l}\text { Appling a high current } \\
\text { through the ink causes the } \\
\text { ink to vaporize, resulting in } \\
\text { ink ejection. }\end{array}$ & $\begin{array}{l}\text { The high current vaporizes } \\
\text { the ink in the nozzle, creat- } \\
\text { ing a pressure pulse. Simi- } \\
\text { lar to bubble jet, vaporizing } \\
\text { the ink requires an ink that is } \\
\text { stable under these conditions. } \\
\text { To maintain a broad material } \\
\text { spectrum, selective vaporiza- } \\
\text { tion is not desired. }\end{array}$ \\
\hline & & Continued on next page \\
\hline
\end{tabular}


Table 2.1 - continued from previous page

\begin{tabular}{|c|c|c|}
\hline $\begin{array}{l}\text { Droplet genera- } \\
\text { tion principle }\end{array}$ & Short description & Applicable \\
\hline $\begin{array}{l}\text { Piezoelectric } \\
\text { direct pressure } \\
\text { pulse }\end{array}$ & $\begin{array}{l}\text { Droplets are propelled out of } \\
\text { a nozzle by means of a shock- } \\
\text { wave generated by a piezo el- } \\
\text { ement. }\end{array}$ & Possible. \\
\hline $\begin{array}{l}\text { Focused acoustic } \\
\text { beam ejection } \\
\text { (Acoustic ink } \\
\text { printing) }\end{array}$ & $\begin{array}{l}\text { Similar to piezoelectric direct } \\
\text { pressure pulse, however a } \\
\text { special focussing lens design } \\
\text { is used to increase shock- } \\
\text { wave efficiency. }\end{array}$ & Possible. \\
\hline $\begin{array}{lr}\text { Flex } r & \text { tensional } \\
\text { aperture } & \text { plate } \\
\text { inkjet } & \end{array}$ & $\begin{array}{l}\text { Similar to piezoelectric di- } \\
\text { rect pressure pulse, however } \\
\text { the nozzle itself is moved } \\
\text { to cause the droplets to be } \\
\text { ejected. }\end{array}$ & Possible. \\
\hline $\begin{array}{l}\text { Thermal electro- } \\
\text { static inkjet }\end{array}$ & $\begin{array}{l}\text { Similar to electro hydrody- } \\
\text { namic inkjet, however each } \\
\text { nozzle can be selectively } \\
\text { heated to change fluid viscos- } \\
\text { ity enabling jet creation on } \\
\text { demand. }\end{array}$ & $\begin{array}{l}\text { This principle is based on } \\
\text { special rheological behaviour } \\
\text { of the ink, so not ink indepen- } \\
\text { dent. }\end{array}$ \\
\hline $\begin{array}{l}\text { Liquid ink fault } \\
\text { tolerant (LIFT) } \\
\text { process }\end{array}$ & $\begin{array}{l}\text { Similar to thermal electro- } \\
\text { static inkjet, however based } \\
\text { mainly on change of surface } \\
\text { tension to enable jet creation } \\
\text { on demand. }\end{array}$ & $\begin{array}{l}\text { This principle is based on } \\
\text { special rheological behaviour } \\
\text { of the ink, so not ink indepen- } \\
\text { dent. }\end{array}$ \\
\hline $\begin{array}{l}\text { Electro- } \\
\text { rheological } \\
\text { fluid inkjet }\end{array}$ & $\begin{array}{l}\text { Similar to thermal electro- } \\
\text { static inkjet, however using } \\
\text { an electric field instead of } \\
\text { heating together with a spe- } \\
\text { cial electro-rheological fluid } \\
\text { which changes fluid proper- } \\
\text { ties depending on the pres- } \\
\text { ence of an electric field. }\end{array}$ & $\begin{array}{l}\text { This principle is based on } \\
\text { special rheological behaviour } \\
\text { of the ink, so not ink indepen- } \\
\text { dent. }\end{array}$ \\
\hline
\end{tabular}


Table 2.1 - continued from previous page

\begin{tabular}{|l|l|l|}
\hline $\begin{array}{l}\text { Droplet genera- } \\
\text { tion principle }\end{array}$ & Short description & Applicable \\
\hline $\begin{array}{l}\text { Thermal- } \\
\text { rheological } \\
\text { fluid inkjet }\end{array}$ & $\begin{array}{l}\text { Similar to thermal electro- } \\
\text { static inkjet, however here a } \\
\text { constant supply pressure is } \\
\text { used to propel the ink. Selec- } \\
\text { tive heating is used to allow } \\
\text { jet formation. }\end{array}$ & $\begin{array}{l}\text { This principle is based on } \\
\text { special rheological behaviour } \\
\text { of the ink, so not ink indepen- } \\
\text { dent. }\end{array}$ \\
\hline $\begin{array}{l}\text { Topspot micro- } \\
\text { drop ejector }\end{array}$ & $\begin{array}{l}\text { The nozzle assembly is } \\
\text { moved and stopped suddenly } \\
\text { causing the ink to be ejected } \\
\text { from the nozzles. }\end{array}$ & $\begin{array}{l}\text { Only low frequency opera- } \\
\text { tion is possible due to total } \\
\text { volume displacement. }\end{array}$ \\
\hline
\end{tabular}

Several droplet generation principles appear to be promising, labelled "possible" in table 2.1. For further comparison, the remaining principles can be split up in two main categories:

$\diamond$ Continuous inkjet based systems.

$\diamond$ Drop-on-demand inkjet based systems.

Other specific details can mainly be found in the way the break-up and/or ejection energy is delivered to the fluid.

It's straight forward that ejecting a highly viscous material out of a small nozzle requires a high pressure difference. With a drop-on-demand system the total energy required to eject the material and create a droplet has to be delivered by the actuation principle. If more energy is required in general a larger actuation mechanism is necessary. With a single nozzle drop-on-demand system this can still be realized; however in the scale up to a multi nozzle system difficulties can be foreseen.

A continuous system on the other hand splits up the effort. The energy necessary to eject the fluid jet is provided by the pressure delivered by the fluid supply. The actuation element only needs to generate a perturbation on the fluid jet leading to jet break-up, resulting in drop formation. Thus the choice for a continuous system seems more logical. A second advantage can be derived if the used ink behaves shear thinning, resulting in a difference between the pressure required to start up a fluid jet (low shear situation) compared to the pressure necessary to maintain fluid flow through the nozzle (high shear situation). Thus a continuous system needs a lower operating pressure since the start-up effect only needs to be overcome once. If the fluid jet is created the high shear situation remains constant, in contrary to 
a drop-on-demand system which has to start up the fluid motion from low to high shear with every drop ejected. To achieve a certain printing width a single nozzle continuous system can be fitted with a multiple deflection system. Another option to print a certain width in one single pass is to use a multi nozzle system. For a high viscosity drop-on-demand system this seems very challenging, since all nozzles must be actuated separately. With a continuous inkjet system on the other hand all nozzles are continuously operated on the same frequency; one single (large) pressure perturbation system can be designed to operate all nozzles simultaneously.

In table 2.2, an overview of advantages and disadvantages of the several systems is given.

Table 2.2: Advantages an disadvantages of different systems.

\begin{tabular}{|c|c|c|}
\hline Type of system: & Advantages: & Disadvantages: \\
\hline $\begin{array}{l}\text { Single nozzle } \\
\text { drop-on-demand } \\
\text { inkjet }\end{array}$ & $\begin{array}{l}\text { - Simple control system pos- } \\
\text { sible } \\
\text { - One nozzle (only one noz- } \\
\text { zle can clog, which is directly } \\
\text { noted, and measures can be } \\
\text { taken) }\end{array}$ & $\begin{array}{l}\text { - High start-up pressure with } \\
\text { every ejected droplet } \\
\text { - Difference in drop size and } \\
\text { drop speed depending on op- } \\
\text { erating frequency (thus de- } \\
\text { pending on image printed) } \\
\text { - Drying of the ink inside } \\
\text { the nozzle if stationary (clog- } \\
\text { ging) }\end{array}$ \\
\hline $\begin{array}{l}\text { Multi nozzle } \\
\text { drop-on-demand } \\
\text { inkjet }\end{array}$ & $\begin{array}{l}\text { - Simple control system pos- } \\
\text { sible } \\
\text { - Multi nozzle, enabling high } \\
\text { speed printing }\end{array}$ & $\begin{array}{l}\text { - High start-up pressure with } \\
\text { every ejected droplet } \\
\text { - Difference in drop size and } \\
\text { drop speed depending on op- } \\
\text { erating frequency (thus de- } \\
\text { pending on image printed) } \\
\text { - Drying of ink inside the } \\
\text { nozzle if stationary (clog- } \\
\text { ging) } \\
\text { - Clogging of one single noz- } \\
\text { zle is not immediately no- } \\
\text { ticed, which can lead to re- } \\
\text { jection of the final product } \\
\text { - Clogged nozzle difficult to } \\
\text { reopen }\end{array}$ \\
\hline & & Continued on next page \\
\hline
\end{tabular}


Table 2.2 - continued from previous page

\begin{tabular}{|c|c|c|}
\hline Type of system: & Advantages: & Disadvantages: \\
\hline $\begin{array}{l}\text { Single nozzle } \\
\text { multiple deflec- } \\
\text { tion continuous } \\
\text { inkjet }\end{array}$ & $\begin{array}{l}\text { - Lower operating pressure } \\
\text { possible (only peak at start- } \\
\text { up) } \\
\text { - One nozzle (only one noz- } \\
\text { zle can clog, which is directly } \\
\text { noted, and measures can be } \\
\text { taken) } \\
\text { - Stable drop size } \\
\text { - Stable drop speed } \\
\text { - Due to continuous opera- } \\
\text { tion, no drying of nozzle }\end{array}$ & $\begin{array}{l}\text { - Complex system } \\
\text { - Lower accuracy due to long } \\
\text { throw distance }\end{array}$ \\
\hline $\begin{array}{l}\text { Multi nozzle } \\
\text { binary deflection } \\
\text { continuous inkjet }\end{array}$ & $\begin{array}{l}\text { - Low operating pressure } \\
\text { possible (only peak at start- } \\
\text { up) } \\
\text { - Stable drop size } \\
\text { - Stable drop speed } \\
\text { - Due to continuous opera- } \\
\text { tion, no drying of nozzle }\end{array}$ & $\begin{array}{l}\text { - Complex system } \\
\text { - Low accuracy due to long } \\
\text { throw distance } \\
\text { - Clogging of one single noz- } \\
\text { zle is not immediately no- } \\
\text { ticed, which can lead to re- } \\
\text { jection of the final product }\end{array}$ \\
\hline
\end{tabular}

According to the advantages and disadvantages, the design of a single nozzle continuous system seems a logical choice to start with. If this reveals feasible, the step towards a multi nozzle system can be made in the future.

When using a continuous inkjet principle it should be possible to create uniform droplets of a high viscosity fluid as long as the perturbation which is applied is sufficiently dominant. From the requirements four main aspects relate to the perturbation mechanism:

$\diamond$ The actuation mechanism should work independent of the type of fluid (chemical composition independent).

$\diamond$ The actuation mechanism should be able to work at several (high) frequencies, allowing frequency optimization and enabling drop size adjustment.

$\diamond$ The amplitude of the actuation mechanism should be adjustable, enabling control over droplet formation.

$\diamond$ The actuation mechanism should lead to droplet break-up within a reasonable distance from the nozzle, to keep the distance between printhead and substrate as small as possible to maintain droplet positioning accuracy. 
In the following sections first the creation of the perturbation will be discussed in more detail, followed by the key elements of the printhead:

$\diamond$ Principle of actuation.

$\diamond$ The actuation mechanism itself.

$\diamond$ The sealing between actuation mechanism and the ink.

$\diamond$ The nozzle construction.

\section{Principle of actuation:}

An effective vibration has to be initiated on the surface of the fluid jet. Different approaches can be chosen to achieve this. These approaches can be subdivided depending on the location where the vibration is initiated. In table 2.3 an overview is given of these locations, together with advantages and disadvantages:

Table 2.3: Advantages and disadvantages depending on the location of the initiation of vibration.

\begin{tabular}{|l|l|l|l|}
\hline "Where" & "How" & Advantages & Disadvantages \\
\hline $\begin{array}{l}\text { After the ma- } \\
\text { terial has left } \\
\text { the nozzle }\end{array}$ & $\begin{array}{l}\text { The initiation of } \\
\text { the vibration after } \\
\text { the material has left } \\
\text { the nozzle can be } \\
\text { done for instance } \\
\text { by selectively } \\
\text { heating the jet by } \\
\text { means of a pul- } \\
\text { sating laser. The } \\
\text { selective thermal } \\
\text { expansion and pos- } \\
\text { sible adjustment of } \\
\text { surface tension and } \\
\text { viscosity results in } \\
\text { jet break-up }\end{array}$ & $\begin{array}{l}\text { - Expensive laser } \\
\text { equipment neces- } \\
\text { sary } \\
\text { - Possible material } \\
\text { interaction due to } \\
\text { laser irradiation }\end{array}$ \\
$\mid$
\end{tabular}


Table 2.3 - continued from previous page

\begin{tabular}{|c|c|c|c|}
\hline "Where" & "How" & Advantages & Disadvantages \\
\hline $\begin{array}{l}\text { At the nozzle } \\
\text { itself }\end{array}$ & $\begin{array}{l}\text { The nozzle plate } \\
\text { can be subjected } \\
\text { to a vibration it- } \\
\text { self, either in line } \\
\text { with the fluid out- } \\
\text { flow or perpendicu- } \\
\text { lar. Both types in- } \\
\text { troduce a vibration } \\
\text { in the fluid }\end{array}$ & $\begin{array}{l}\text { - Simple fluid lay- } \\
\text { out, the total print- } \\
\text { head might be actu- } \\
\text { ated }\end{array}$ & $\begin{array}{l}\text { - Actuating the } \\
\text { complete printhead } \\
\text { possibly limits } \\
\text { the actuation fre- } \\
\text { quency due to } \\
\text { relatively high } \\
\text { moving mass } \\
\text { - Nozzle location } \\
\text { not completely } \\
\text { fixed/accurate }\end{array}$ \\
\hline $\begin{array}{l}\text { Before the } \\
\text { material } \\
\text { enters } \\
\text { nozzle }\end{array}$ & $\begin{array}{l}\text { An actuating mem- } \\
\text { ber can be inserted } \\
\text { in the fluid chan- } \\
\text { nel right before the } \\
\text { nozzle }\end{array}$ & $\begin{array}{l}\text { - Actuating mem- } \\
\text { ber can be larger } \\
\text { than jet size }\end{array}$ & $\begin{array}{l}\text { - Vibration has to } \\
\text { travel through the } \\
\text { material, through } \\
\text { the pressure drop } \\
\text { of the nozzle }\end{array}$ \\
\hline
\end{tabular}

Each perturbation location has specific options how the jet can be influenced. After the nozzle, surface and viscosity modifications can be achieved by for instance laser agitation.

If the perturbation is initiated by the nozzle itself, for instance by an inline motion of the nozzle, the resulting perturbation is basically the jet speed fluctuation as a result of the nozzle movement, based on the assumption that the outflow speed out of the nozzle remains constant.

If the perturbation is initiated before the material enters the nozzle, both regimes can be studied. If the vibrating member is relatively far away from the nozzle opening the vibration will only cause a pressure wave delivering pressure variations (sound wave). If the distance between the nozzle and the vibrating member decreases, the effect on the material flow becomes more significant (squeezing). Due to the possibility to vary between both regimes it is chosen to initiate the vibration before the material enters the nozzle. A schematic representation of the vibrating mechanism is represented in figure 2.1 .

\section{Actuation mechanism:}

The vibrating member can be actuated with several mechanisms. Basically a system can be chosen where a rotating movement is transformed into a translating movement (illustrated in figure 2.2) or a system can be chosen where a translating move- 


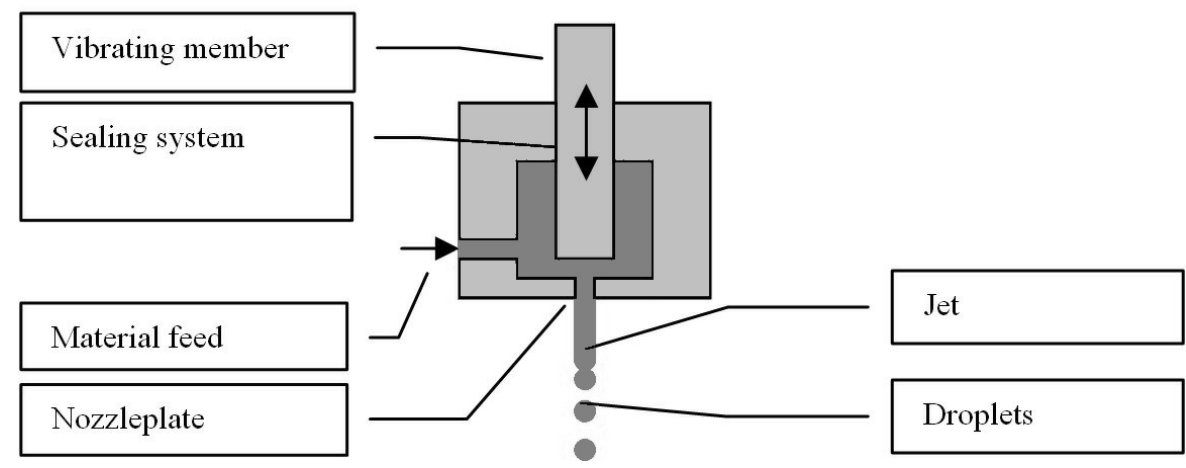

Figure 2.1: Schematic representation of the actuating mechanism.

ment is created instantaneously by means of a voice coil or piezo element (figure 2.3). In table 2.4 an overview of advantages and disadvantages of the different systems is given. In an experimental situation the flexibility in waveform, which the piezo and voice coil actuator principles supply, is preferred. The piezo electric actuation seems to be the most flexible solution, whilst still being able to realise the forces necessary. The only drawback is its disability to operate at high temperatures if commonly used piezo elements are used. These may not exceed $140^{\circ} \mathrm{C}$. For most printing applications this will not be a limiting factor, however, if this is the case, special high temperature piezo elements are available nowadays with Curie temperatures up to $820^{\circ} \mathrm{C}$, sustaining operating temperatures of $590^{\circ} \mathrm{C}$ [33].

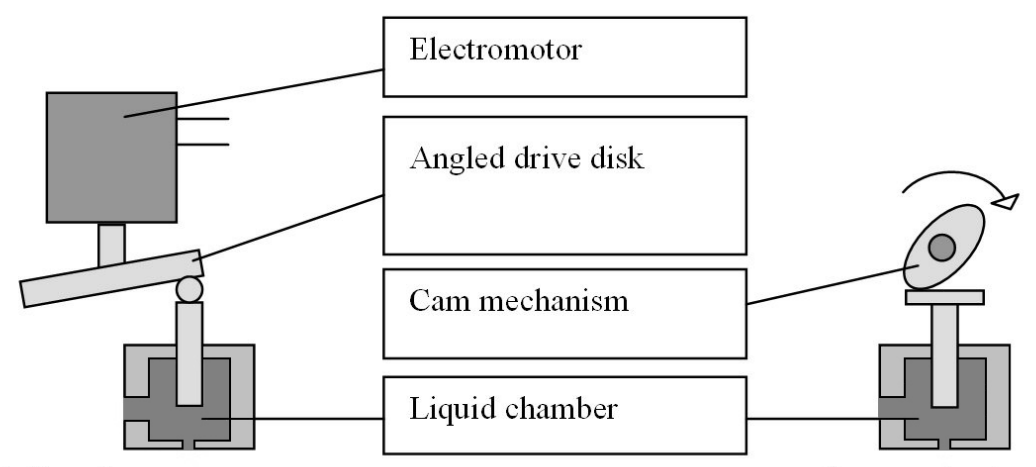

Rotating disc

Cam mechanism

Figure 2.2: Schematic representation of mechanical actuating mechanisms. 


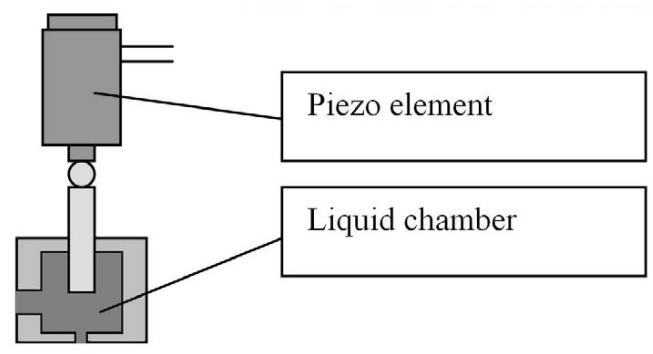

Figure 2.3: Schematic representation of a piezo based actuating mechanism.

Table 2.4: Advantages and disadvantages of different types of actuating principles.

\begin{tabular}{|c|c|c|}
\hline Type of system: & Advantages & Disadvantages \\
\hline $\begin{array}{ll}\text { Rotating } & \text { disc } \\
\text { (figure 2.2) } & \end{array}$ & $\begin{array}{l}\text { - High temperature operation } \\
\text { possible } \\
\text { - Possibility to actuate down- } \\
\text { wards as well as upwards } \\
\text { movement }\end{array}$ & $\begin{array}{l}\text { - Fixed pulse waveform, no } \\
\text { variation in pulse shape pos- } \\
\text { sible without machining a } \\
\text { different disc } \\
\text { - Limitation in maximum op- } \\
\text { erating frequency } \\
\text { - Wear sensitive especially at } \\
\text { higher rotational speeds }\end{array}$ \\
\hline $\begin{array}{l}\text { Cam mechanism } \\
\text { (figure 2.2) }\end{array}$ & $\begin{array}{l}\text { - High temperature operation } \\
\text { possible }\end{array}$ & $\begin{array}{l}\text { - Fixed pulse, no variation in } \\
\text { pulse shape possible without } \\
\text { machining a different disc } \\
\text { - Limitation in maximum op- } \\
\text { erating frequency } \\
\text { - Wear sensitive especially at } \\
\text { higher rotational speeds }\end{array}$ \\
\hline $\begin{array}{l}\text { Voice coil actua- } \\
\text { tion }\end{array}$ & $\begin{array}{l}\text { - Variable pulse shape control } \\
\text { - High frequencies achiev- } \\
\text { able } \\
\text { - Wear insensitive }\end{array}$ & - Limited actuation force \\
\hline $\begin{array}{l}\text { Piezo actuation } \\
\text { (figure 2.3) }\end{array}$ & $\begin{array}{l}\text { - Variable pulse shape control } \\
\text { - High frequencies achiev- } \\
\text { able } \\
\text { - Wear insensitive }\end{array}$ & $\begin{array}{l}\text { - Limited operating tempera- } \\
\text { ture }\end{array}$ \\
\hline
\end{tabular}




\section{Sealing system:}

The vibrating member will be translated by the piezo element over a short distance. A sealing system has to be applied to prevent fluid from escaping. The sealing mechanism has to be able to withstand the high pressures expected in the printhead when processing higher viscous materials. The following requirements can be summarized:

$\diamond$ Resistant to high pressure ( $\sim 200$ bar).

$\diamond$ Free of hysteresis.

$\diamond$ Serve as guiding means for the vibrating member.

$\diamond$ Relatively large displacement possible, to enable setting of distance between vibrating member and outflow opening.

$\diamond$ Compact.

Basically two options arise:

$\diamond$ Using a membrane construction.

$\diamond$ Using a dynamic seal (o-ring or alike).

Both systems have their unique advantages and disadvantages as displayed in table 2.5.

Table 2.5: Advantages and disadvantages of the sealing system.

\begin{tabular}{|l|l|l|}
\hline Type of system: & Advantages & Disadvantages \\
\hline Membrane & $\begin{array}{l}- \text { Hysteresis free movement } \\
\text { possible } \\
\text { - Functional at high tempera- } \\
\text { tures }\end{array}$ & - Limited in movement \\
\hline O-ring & $\begin{array}{l}\text { Simple construction of } \\
\text { small size }\end{array}$ & $\begin{array}{l}\text { - Limited in maximum oper- } \\
\text { ating temperature } \\
\text { - Possible hysteresis effect } \\
\text { - Possible wear in sliding mo- } \\
\text { tion }\end{array}$ \\
\hline
\end{tabular}

Although from this schematic overview the membrane seems to have quite some advantages, the limited movement is undesired in an experimental setup. The flexibility of possible settings leads to favouring the o-ring solution at this point. If eventually the setting can be fixed and the system can be miniaturised the membrane solution becomes an interesting option again. Figure 2.4 gives a schematic representation of the sealing mechanism. The vibrating member is enclosed by two seals 
forming a guiding mechanism. The seals selected have a large cross-section diameter. This way the small movement the vibrating member performs will remain in the elastic deformation range of the seal, preventing possible hysteresis effects.

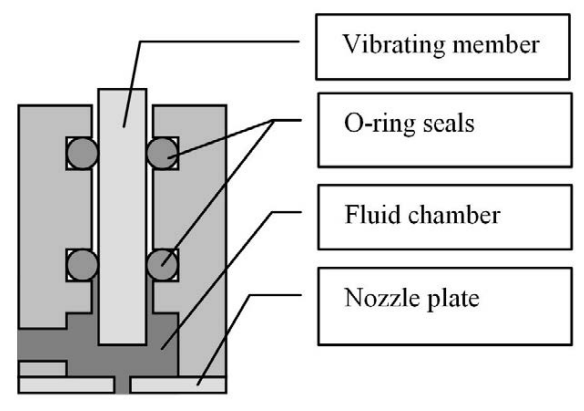

Figure 2.4: Overview of sealing solution.

\section{Nozzle:}

Not only the seals have to be capable to withstand the resulting pressures, also the nozzle itself should be constructed not to break under pressure. Since it is desirable to test several different nozzle sizes and geometries, the nozzle should be easily manufacturable. Two main dimensions determine the manufacturability, namely the nozzle diameter, and the length of the nozzle. Two basic constructions can be considered, either a single element system (left figure 2.5) where the nozzle is created in a thinned section or a two element combination (right figure 2.5), where a support plate withstands the pressure inside the printhead and the nozzle can be designed as a thin foil. One can imagine the size of the hole in the nozzle support plate to be of influence on the systems performance, the unsupported part of the nozzle foil might flex and vibrate under the applied pressure fluctuations. However this last layout remains preferred due to its reduced cost per nozzle.

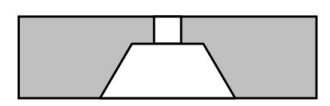

Single element

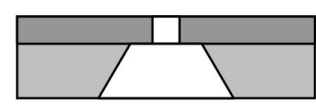

Two elements

Figure 2.5: Schematic nozzle layout. 


\subsubsection{Theoretical achievability of high viscosity jetting}

In the previous paragraph the printhead design was described in general. To define the design in more detail it is necessary to gain more insight in the processes and the effect of dimensioning on the efficiency of the system. One has to consider whether it is achievable to realize stable drop formation using a higher viscous material. The phenomena that occur with the break-up process of a fluid jet have to be studied in more detail. If the printhead geometry and nozzle size are chosen, two main parameters determine the resulting droplet volume, namely the amount of material processed through the nozzle and the frequency in which the resulting jet breaks up into separate droplets. Since creation of uniform drops is required for a well performing inkjet system, it is important to investigate whether stable droplet break-up can be achieved in the parameter space available.

Therefore first the natural jet break-up will be described, followed by the operating window which can be addressed using forced jet break-up, concluding with a prediction of the expected vibration depending on system dimensions to provide a guideline for the final design.

\section{Natural jet break-up:}

In a series of papers Lord Rayleigh [34-36] revisited the work of Plateau [37] describing the break-up process of liquid jets, showing that vibrations with a wavelength $\lambda$ larger then the circumference of the jet with radius $r_{j}$ cause the jet to break-up into droplets, $\lambda>2 \cdot \pi \cdot r_{j}$. This equation can also be expressed on the basis of the wave number $k$ as $k \cdot r_{j}<1$, where $k=(2 \cdot \pi) / \lambda$. The disturbance with the largest growth speed dominates the break-up process and is also known as the natural break-up frequency. Equation 2.1 gives an approximation of the growth speed as a function of the wave number.

$$
\omega=\sqrt{\frac{\sigma_{0}}{2 \rho \cdot r_{j}^{3}} k^{2} \cdot r_{j}^{2}\left(1-k^{2} \cdot r_{j}^{2}\right)}
$$

here $\omega$ represents the growth speed of the disturbance, $\sigma_{0}$ the surface tension and $\rho$ the specific density of the fluid. From this growth speed the length of the jet before break-up can be calculated based on the assumption that the fluid jet breaks up when the amplitude $\alpha$ of the dominant disturbance equals the jet radius $r_{j}$ according to $L=u / \omega \cdot \ln \left(r_{j} / \alpha_{0}\right)$ where $L$ represents the break-up length, $u$ the jet speed and $\alpha_{0}$ the initial disturbance [38].

Based on experimental evidence [38] the factor $\ln \left(r_{j} / \alpha_{0}\right)$ for an undisturbed jet can be approximated by $\ln \left(r_{j} / \alpha_{0}\right)=12$ for $O h>0.015$ and $\ln \left(r_{j} / \alpha_{0}\right)=6.5 \cdot(O h)^{-0.25}$ 
for $O h<0.015$. Here $O h=\sqrt{W e} / R e=\mu / \sqrt{\left(\sigma_{0} \cdot \rho \cdot d_{n}\right)}$ is the Ohnesorge number where $\mu$ represents the fluid viscosity and $d_{n}$ the nozzle diameter.

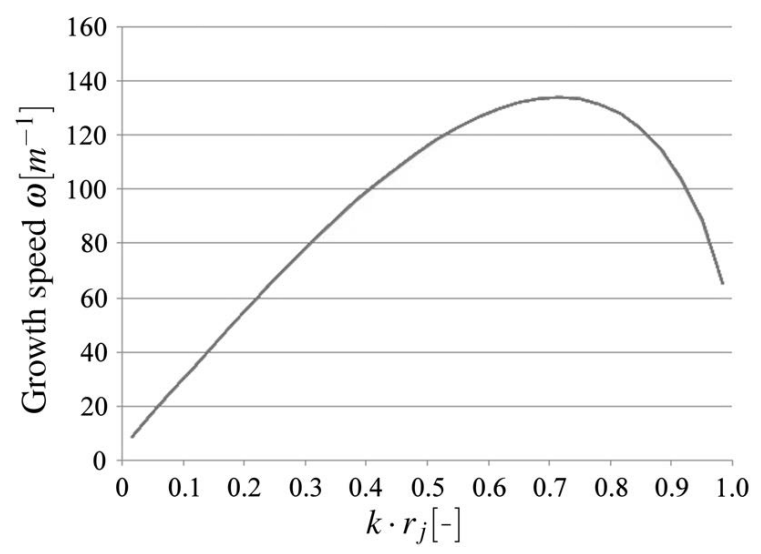

Figure 2.6: Growth speed of a water jet exiting a $50 \mu \mathrm{m}$ nozzle as a function of $k \cdot r_{j}$.

Figure 2.6 shows the growth speed as a function of $k \cdot r_{j}$, showing a maximum at $k \cdot r_{j}=0.696$, resulting in a maximum instability frequency $f_{i}$ according to $f_{i}=$ $u /\left(9.016 \cdot r_{j}\right)$ also known as natural break-up frequency.

\section{Forced jet break-up:}

The top of the graph in figure 2.6 is rather flat, resulting in the ability to induce break-up at frequencies near the maximum by giving them a head-start by actuation at this required frequency. In literature several operational boundaries are given with respect to allowable jet speed and the flexibility in jet break-up frequency, describing an operating window for stable droplet creation as represented in figure 2.7. These boundaries are derived from experiments and system dependent, therefore these curves have to be considered as a guideline of what one can expect in terms of performance, not as a fixed boundary.

For the lower speed limit different approximations can be found in literature. Clanet et al. related the lower speed limit to a critical Weber number $\left(W e_{c}\right)$ [39]. Predicting stable jetting when $W e=\left(\rho \cdot u^{2} \cdot d_{n}\right) / \sigma_{0}>W e_{c}$ where $W e_{c}$ is dependent on specific density and surface tension, but independent on fluid viscosity according to

$$
W e_{c}=4 \frac{B o_{o}}{B o}\left[1+K B o_{o} B o-\sqrt{\left(1+K B o_{o} B o\right)^{2}-1}\right]^{2}
$$

where $B o=\sqrt{\rho \cdot g \cdot d_{n}^{2} / 2 \sigma}$ is the Bond number with respect to the inside diameter 


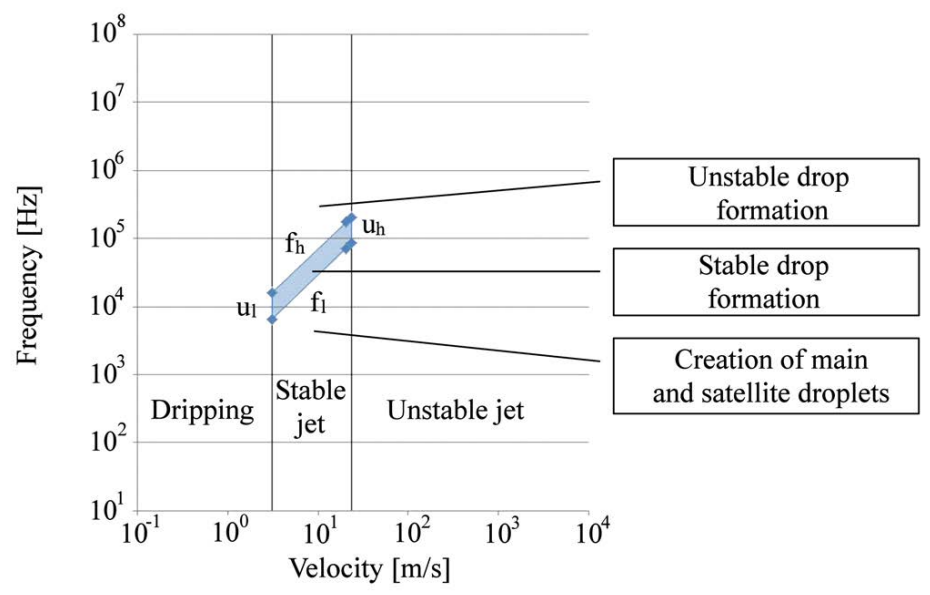

Figure 2.7: Region of uniform drop formation of water.

of the nozzle $d_{n}$ and $B o_{o}=\sqrt{\rho \cdot g \cdot d_{o}^{2} / 2 \sigma}$ with respect to the outside diameter of the nozzle $d_{o}$ and $K$ is a constant, in this case 0.37 .

Matsushits et al. [40] heuristically derived that the lower speed limit for low viscous $\left(0-50 \cdot 10^{-3} \mathrm{~Pa} \cdot \mathrm{s}\right)$ Newtonian fluids can be represented by

$$
u_{l}=3 \cdot(O h)^{-0.96} \cdot\left(\frac{\mu}{d_{n} \cdot \rho}\right)
$$

and for viscous Newtonian fluids $\left(30-600 \cdot 10^{-3} \mathrm{~Pa} \cdot \mathrm{s}\right)$ the lower speed limit can be described by

$$
u_{l}=1.4 \cdot(O h)^{-1.33} \cdot\left(\frac{\mu}{d_{n} \cdot \rho}\right)
$$

Contrary to Clanet et al. the speed limit described by Matsushits et al. is related to fluid viscosity of the jet.

If the speed is increased above the upper speed limit, the flow inside the nozzle will no longer behave in a laminar way. After leaving the nozzle the entrained air becomes turbulent causing even more instability. The upper speed limit ( $u_{h}$ in figure 2.7) can be approximated by [41]

$$
u_{h}=325 \cdot(O h)^{-0.28} \cdot\left(\frac{\mu}{d_{n} \cdot \rho}\right)
$$


and [42]

$$
u_{h}=300 \cdot(O h)^{-0.33} \cdot\left(\frac{\mu}{d_{n} \cdot \rho}\right)
$$

Sakai et al. [42] described the lower and upper frequency boundaries $\left(f_{l}\right.$ and $f_{h}$ in figure 2.7) based on experimental results using a longitudinal moving nozzle. For the creation of uniform drops of Newtonian liquids with a viscosity between $1 \cdot 10^{-3}$ to $50 \cdot 10^{-3} \mathrm{~Pa} \cdot \mathrm{s}$ these limits can be represented by $[43,44]$

$$
\begin{gathered}
f_{l}=0.11 \cdot(R e)^{-0.066} \cdot(W e)^{0.17} \cdot\left(\frac{u}{d_{n}}\right) \\
f_{h}=0.18 \cdot(R e)^{0.031} \cdot(W e)^{0.12} \cdot\left(\frac{u}{d_{n}}\right)
\end{gathered}
$$

For higher viscous Newtonian fluids (between 30 and $600 \cdot 10^{-3} \mathrm{~Pa} \cdot \mathrm{s}$ ) Sakai et al. described a different limit also based on the amplitude of the longitudinal moving nozzle $\delta$ [44].

$$
\begin{aligned}
& f_{l}=0.059 \cdot(\operatorname{Re})^{0.325} \cdot(W e)^{0.132} \cdot\left(\frac{\delta}{d_{n}}\right)^{0.859} \cdot\left(\frac{u}{d_{n}}\right) \\
& f_{h}=0.106 \cdot(\operatorname{Re})^{0.378} \cdot(W e)^{0.077} \cdot\left(\frac{\delta}{d_{n}}\right)^{0.863} \cdot\left(\frac{u}{d_{n}}\right)
\end{aligned}
$$

Compared to conventional continuous inkjet systems, the system described in this thesis is aimed for processing higher viscous materials. Therefore two areas are visualized in figure 2.8 . The operating window using:

$\diamond 2$-Propanol, also having a low viscosity, however combined with a relatively low surface tension.

$\diamond$ Test oil with a viscosity of $200 \mathrm{mPa} \cdot \mathrm{s}$.

It can be seen that the area of uniform drop formation for more viscous fluids alters; the flexibility with respect to allowable operating frequencies becomes smaller. However the range where a stable jet can be formed increases. It can be doubted whether stable drop formation is realistic at high frequencies since maintaining the needed amplitude at these extreme frequencies becomes challenging.

Altogether, it is assumed that if the high viscous fluid can be ejected from the nozzle and the system can be designed in such a way that a perturbation can be created on the surface of the fluid jet, there is a chance that stable droplets can be generated albeit in an altered process window compared to low viscous materials. 


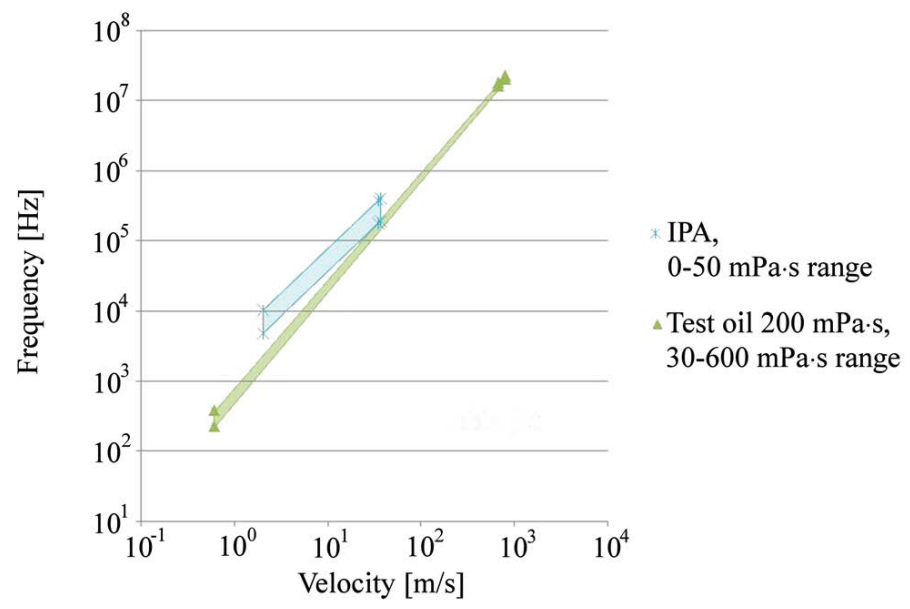

Figure 2.8: Region of uniform drop formation for 2-propanol and a $200 \mathrm{mP} \cdot \mathrm{s}$ test oil.

\section{Prediction of the expected vibration depending on system dimensions:}

To achieve the maximum chance for an effective perturbation the choice of system dimensions is critical. In the general design in paragraph 2.1.1 no dimension was selected. In the following section an analytical model is described to achieve insight into which dimensions are required to develop a significant fluctuation. A lot of literature is available on the preferred frequency and jet speed domain as described in the previous section. However, only experimental approximations are available on the amount of perturbation necessary. Typical commercially available continuous inkjet printers use low viscous materials requiring an operating pressure of 0.34 to 3.4 bars depending on the nozzle size. The modulation pressure at the nozzle is in the order of less than 0.14 bars [15]. When aiming at processing higher viscous materials, the operating pressure will raise significantly. It is expected that the modulation pressure needs to rise with the same order of magnitude.

The fluid flow through the printhead is mainly defined by the geometry of the channel between the focussing member and the nozzle plate. In figure 2.9 a close-up thereof is given with respect to an axi-symmetrical coordinate system $r-z$.

When the ratio $h_{\text {gap }} / r_{\text {piezo }}<<1$ (figure 2.9), the lubrication theory can be applied for the fluid flow [45]. As a starting point a Newtonian fluid is assumed. The system is considered isothermal and the mechanical transmission is considered beyond the scope of this thesis. From the axi-symmetrical Navier-Stokes equations the following 


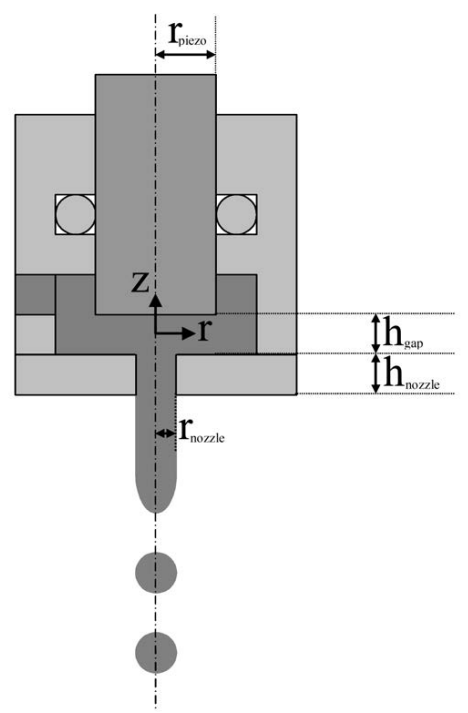

Figure 2.9: The flow channel between piezo-actuator and nozzle plate.

equation of the pressure gradient over the channel can be derived :

$$
\frac{d p}{d r}=\mu \frac{\partial^{2} v_{r}}{\partial z^{2}}
$$

where the pressure gradient is dependent on the fluid viscosity $\mu$ and on the second derivative of the radial velocity $v_{r}$ and thus a function of $r$. Whether the velocity profile can be considered parabolic is dependent on the penetration depth of the occurring shear wave. This penetration depth $x$ is dependent on the viscosity, density and actuation frequency $f$ :

$$
x=\frac{\pi}{\kappa} \quad \text { with } \kappa=\sqrt{\frac{\pi \cdot \rho \cdot f}{\mu}}
$$

Considering a fluid with a viscosity of $500 \mathrm{mPa} \cdot \mathrm{s}$, with a density of $1000 \mathrm{~kg} / \mathrm{m}^{3}$ being processed with an actuation frequency of $20 \mathrm{kHz}$ this leads to a penetration depth of $280 \mu \mathrm{m}$. For approximation of the effects occurring with viscous fluids using small $h_{\text {gap }}$ dimensions a parabolic velocity profile can be used.

To simplify the following equations $h_{\text {gap }}$ (figure 2.9 ) is replaced by $2 h$ since the underlying calculations are based on a coordinate system where the origin of the $z$ coordinate is in the middle of the flow. Assuming a parabolic speed profile between 
piezo-actuator and the nozzle plate, the radial speed $v_{r}$ can be formulated as a function of the average radial speed $\bar{v}_{r}$ :

$$
v_{r}=\frac{3}{2} \bar{v}_{r}\left(1-\left(\frac{z}{h}\right)^{2}\right)
$$

This equation can be entered in the equation for the pressure gradient in the channel. The average radial speed can be calculated by applying conservation of matter on the fluid flow through the channel on position $r$ :

$$
\bar{v}_{r}=-\frac{r \dot{h}}{2 h}-\frac{q_{n o z z l e}}{4 \pi r h}
$$

Here $q_{\text {nozzle }}$ is the mean fluid flow through the nozzle and $v_{\text {piezo }}=-2 \dot{h}$ is the speed of the piezo-actuator. Substitution of 2.14 in 2.13 results in the following equation for the radial speed:

$$
v_{r}=-\frac{3}{2}\left[\frac{r \dot{h}}{2 h}+\frac{q_{\text {nozzle }}}{4 \pi r h}\right]\left(1-\left(\frac{z}{h}\right)^{2}\right)
$$

The explicit expression for the pressure in the channel can be derived by substituting the equation above in the equation for the pressure gradient, integrating the resulting expression over the radius of the piezo-actuator, and finally completing the equations for $h_{\text {gap }}$ and $v_{\text {piezo }}$, resulting in:

$$
p(r)= \begin{cases}\frac{3 \mu v_{\text {piezo }}}{h_{\text {gap }}^{3}}\left(r_{\text {piezo }}^{2}-r^{2}\right)+\frac{6 \mu}{\pi h_{\text {gap }}^{3}} q_{\text {nozzle }} \ln \left(\frac{r}{r_{\text {piezo }}}\right)+p_{\text {pump }} & \text { if } r_{\text {nozzle }}<r \leq r_{\text {piezo }} \\ p\left(r_{\text {nozzle }}\right) & \text { if } r \leq r_{\text {nozzle }}\end{cases}
$$

where $r_{\text {nozzle }}$ represents the nozzle radius, $r_{\text {piezo }}$ the radius of the vibrating piezo rod and $p_{\text {pump }}$ represents the pressure of the print fluid at the beginning of the channel between piezo-actuator and the nozzle plate.

If as a first order approximation a Hagen-Poiseuille flow can be assumed, the flow through the nozzle can be described by the following equation:

$$
p\left(r_{\text {nozzle }}\right)-p_{0} \cong \frac{8 \mu h_{\text {nozzle }}}{\pi r_{\text {nozzle }}^{4}} q_{\text {nozzle }}
$$

where $p_{0}$ represents the surrounding pressure and $h_{\text {nozzle }}$ the length of the nozzle hole. 
This way the variation in fluid flow through the nozzle can be described as follows:

$$
q_{\text {nozzle }} \cong \frac{\frac{3 \mu v_{\text {piezo }}}{h_{g \text { app }}^{3}}\left(r_{\text {piezo }}^{2}-r_{\text {nozzle }}^{2}\right)+\left(p_{\text {pump }}-p_{0}\right)}{\frac{8 \mu h_{\text {nozzle }}}{\pi r_{\text {nozzle }}^{4}}-\frac{6 \mu}{\pi h_{\text {gap }}^{3}} \ln \left(\frac{r_{\text {nozzle }}}{r_{\text {piezo }}}\right)}
$$

The force necessary to realise the movement can be calculated by integrating the pressure over the surface of the piezo-actuator, resulting in the following equation:

$$
\begin{aligned}
F_{\text {piezo }} \cong & \pi r_{\text {nozzle }}^{2}\left(p\left(r_{\text {nozzle }}\right)-p_{\text {pump }}\right) \\
& +\int_{r_{\text {nozzle }}}^{r_{\text {piezo }}} 2 \pi r\left[\frac{3 \mu v_{\text {piezo }}}{h_{\text {gap }}^{3}}\left(r_{\text {piezo }}^{2}-r^{2}\right)+\frac{6 \mu}{\pi h_{\text {gap }}^{3}} q_{\text {nozzle }} \ln \left(\frac{r}{r_{\text {piezo }}}\right)\right] \mathrm{d} r
\end{aligned}
$$

Within the model general dimensions and fluid parameters can be varied, a constant pressure at the inlet of the printhead is assumed. Depending on the chosen gap between focussing member and nozzle plate a certain mean flow through the nozzle results dependent on the pressure chosen. To achieve comparable data the pressure is set to result in a mean fluid flow of $2.5 \mathrm{ml} / \mathrm{min}$, leading to equal sized droplets being produced if the frequency and nozzle size are held constant. It can already be noted that the high viscosity requires a significantly different printhead. A mean supply pressure in the order of 70 bars is necessary, compared to only a few bars for conventional printheads generating equal sized droplets. When choosing the dimension of the vibrating member one has to keep in mind a large vibrating member will have a direct influence on the power requirements for the actuating mechanism with these high pressures. The larger the vibrating member the less displacement of the vibrating member can be expected when using the same driving energy. Therefore it is chosen to design the vibrating mechanism as small as possible still using conventional manufacturing methods. A good starting point is the availability of seals. To achieve a system which can withstand aggressive materials Kalrez ${ }^{\circledR}$ seals are selected for the design. These seals can be manufactured to customer specification; however a limited number of standard sizes are available. Therefore a $3.3 \mathrm{~mm}$ seal with a cross-section diameter of $2.4 \mathrm{~mm}$ from the standard product line is taken, defining the basic dimension of the system.

It is assumed that with these small dimensions the piezo element will be able to follow the driving waveform. The piezo element is assumed to perform a sine wave motion according to:

$$
h_{\text {gap }}(t)=h_{\text {gap }, 0}+a_{\text {piezo }} \cdot \sin (r \pi f t)
$$

where $h_{\text {gap }, 0}$ represents the initial distance between actuator and the nozzle, $a_{\text {piezo }}$ the piezo amplitude and $f$ the actuation frequency. 
Table 2.6 gives an overview of four different simulations with their resulting pressure fluctuations. The graphs in figure 2.10 represent the pressure distribution between the vibrating member and the nozzle plate as a function of time and distance from the nozzle. The simulations will be discussed one by one.

Simulation 1: A typical parameter selection using a piezo amplitude of $15 \mu \mathrm{m}$ and a relative large distance between the vibrating member and the nozzle plate of 500 $\mu \mathrm{m}$ as a starting point. The resulting pressure fluctuation under the vibrating member is $1.99 \%$ of the supply pressure (between 61.55 and 62.79 bar). The flow fluctuates between 2.48 and $2.52 \mathrm{ml} / \mathrm{min} ; 1.99 \%$ of the mean flow.

Simulation 2: The parameter most debatable in the first simulation is the distance the vibrating member will actually travel during actuation. A typical piezo element can be chosen to have a maximum travel of $30 \mu \mathrm{m}$, however when operating the piezo element dynamically the actual achievable travel might be significantly lower. This can however be compensated by decreasing the distance between the vibrating member and nozzle plate. Therefore in simulation 2 the amplitude is reduced to $15 \mathrm{~nm}(1000 \mathrm{x}$ less than in simulation 1). By reducing the distance between vibrating member and nozzle plate to $50 \mu \mathrm{m}$ (10x less) a pressure fluctuation of nearly the same magnitude is observed, namely $1.66 \%$ pressure fluctuation (between 61.65 and 62.69 bar). Here the pump pressure is increased to 74.00 bar to maintain an equal mean fluid flow of $2.50 \mathrm{ml} / \mathrm{min}$. The flow fluctuates between 2.48 and $2.52 \mathrm{ml} / \mathrm{min}$; a fluctuation of $1.66 \%$ as well.

Simulation 3: When the piezo element is capable of a higher performance and a vibration amplitude of $0.1 \mu \mathrm{m}$ is achievable the resulting pressure fluctuation will be in the same order of magnitude with respect to the feed pressure as compared to conventional low pressure systems. Simulation 3 shows $11.09 \%$ pressure fluctuation (between 58.72 and 65.62 bar). The flow fluctuates between 2.36 and $2.64 \mathrm{ml} / \mathrm{min}$. Although it can be argued whether the pressure at the edge of the vibrating member is constant as the model takes as a stating point, the model shows that if the distance between vibrating member and the nozzle plate can be varied the system has a large amount of flexibility in order to cope with the performance of the vibration mechanism.

In the previous calculations the construction was considered rigid, resulting in no bending as a result of the applied pressures. When using a thin nozzle plate with a nozzle support it can be expected that the thin nozzle foil will flex under the applied 
pressures. To achieve insight in this effect, the previous model is extended to take the bending of the unsupported nozzle into account using Roark's formulations for stress and strain [46]. The bending of the nozzle is calculated based on the approximation that an equal load is exerted on the nozzle surface. Therefore first the mean pressure underneath the vibrating member as a function of time is calculated. The resulting pressure is, however, dependent on the nozzle deformation. So an iterative process is started, recalculating the mean pressure taking the nozzle deformation previously calculated into account. The loop is stopped when the difference between the nozzle deflection profiles drops underneath a set threshold level.

Simulation 4: Using the same parameters as simulation 3 and introducing stainless steel as a nozzle material with a support plate with a central hole of $2 \mathrm{~mm}$ results in a pressure fluctuation of $10.01 \%$ (a decrease of $1.08 \%$ ) between 59.09 and 65.31 bar. The flow fluctuates between 2.38 and $2.63 \mathrm{ml} / \mathrm{min}$. A nice detail is the reduced pump pressure as a result of reduced resistance due to the bending of the nozzle plate. Although a decrease in the performance of the system can be expected, the ease of manufacture of the nozzle plates is such an advantage that this decrease is acceptable.

Table 2.6: Overview of simulation input and results.

\begin{tabular}{|l|l|l|l|l|}
\hline Variable & $\begin{array}{l}\text { Simulation 1 } \\
\text { (figure 2.10a) }\end{array}$ & $\begin{array}{l}\text { Simulation 2 } \\
\text { (figure 2.10b) }\end{array}$ & $\begin{array}{l}\text { Simulation 3 } \\
\text { (figure 2.10c) }\end{array}$ & $\begin{array}{l}\text { Simulation 4 } \\
\text { (figure 2.10d) }\end{array}$ \\
\hline$r_{\text {nozzle }}[\mu \mathrm{m}]$ & 40 & 40 & 40 & 40 \\
\hline$l_{\text {nozzle }}[\mu \mathrm{m}]$ & 300 & 300 & 300 & 300 \\
\hline$h_{\text {gap }, 0}[\mu \mathrm{m}]$ & 500 & $\mathbf{5 0}$ & 50 & 50 \\
\hline$a_{\text {piezo }}[\mu \mathrm{m}]$ & 15 & $15 \cdot 10^{-3}$ & $\mathbf{0 . 1}$ & 0.1 \\
\hline$r_{\text {piezo }}[\mathrm{mm}]$ & 1.65 & 1.65 & 1.65 & 1.65 \\
\hline$Q_{\text {pump }}[\mathrm{ml} / \mathrm{min}]$ & 2.5 & 2.5 & 2.5 & 2.5 \\
\hline$p_{\text {pump }}[\mathrm{bar}]$ & 62.2 & $\mathbf{7 4 . 0}$ & 74.0 & $\mathbf{7 3 . 6}$ \\
\hline$\mu[\mathrm{mPa} \cdot \mathrm{s}]$ & 500 & 500 & 500 & 500 \\
\hline$f_{\text {piezo }}[\mathrm{kHz}]$ & 20 & 20 & 20 & 20 \\
\hline Deflection [] & No & No & No & Yes \\
\hline$r_{\text {support }}[\mathrm{mm}]$ & N.A. & N.A. & N.A. & 1 \\
\hline$e_{\text {modul }}[\mathrm{Pa}]$ & N.A. & N.A. & N.A. & $195 \cdot 10^{9}$ \\
\hline$\Delta P[\%]$ & 1.99 & $\mathbf{1 . 6 6}$ & $\mathbf{1 1 . 0 9}$ & $\mathbf{1 0 . 0 1}$ \\
\hline & & & & \\
\hline
\end{tabular}




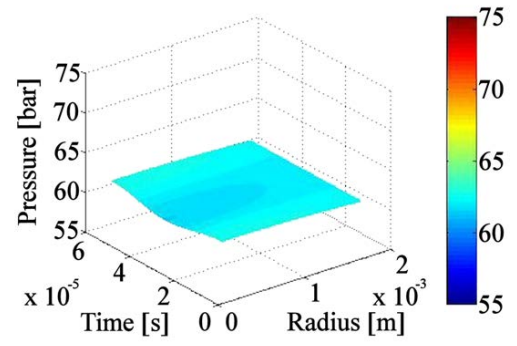

(a) Simulation 1

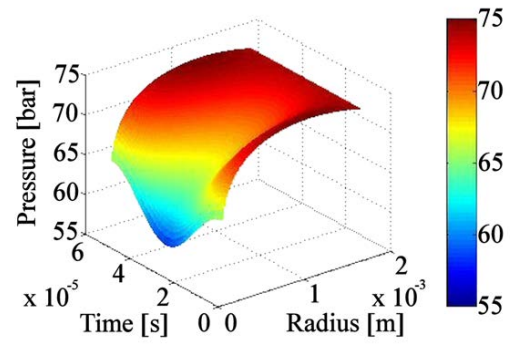

(c) Simulation 3

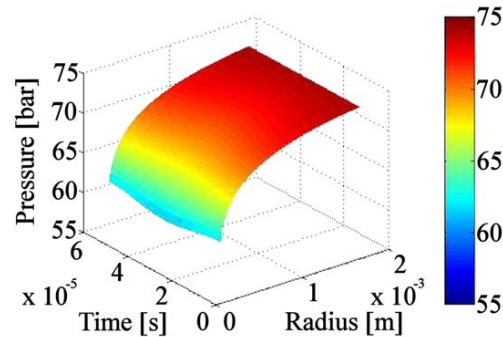

(b) Simulation 2

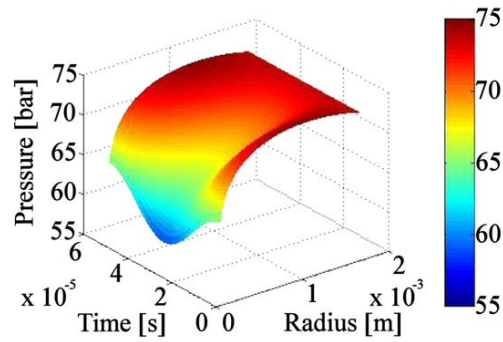

(d) Simulation 4

Figure 2.10: Graphical overview of simulation results.

Finally looking back at the initial starting point from the model it was stated that the lubrication theory can be applied for the fluid flow when the ratio between $h_{\text {gap }} / r_{\text {piezo }}<<1$ [45]. In the chosen examples the system is set between the ratio of $0.03-0.3$. Although 0.3 is not much smaller than 1 , it is assumed that the lubrication theory can still be used as an approximation.

\subsubsection{Final design}

The combination of selections from the previous paragraphs leads to a design as shown in figure 2.11. Two generations of the printhead are shown, the first generation used a two thread design to control the distance between the bottom of the vibrating element and the nozzle plate, the second generation changed to a fine thread design to generate a more compact system (figure 2.12). 

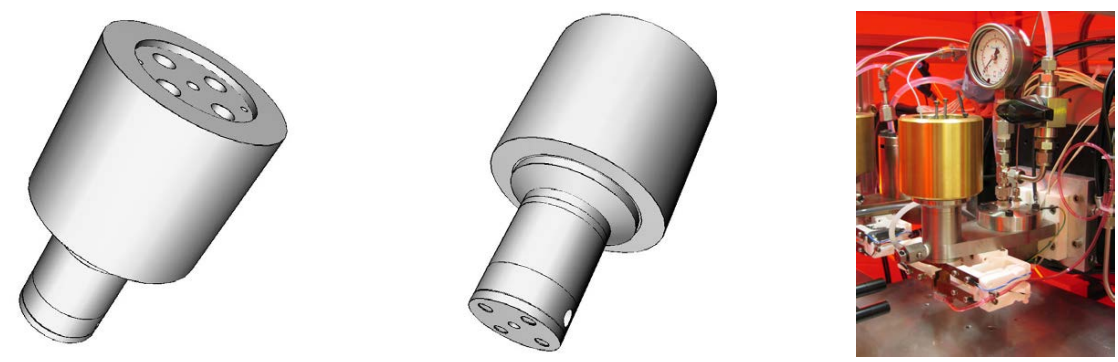

Figure 2.11: First generation High Viscosity Inkjet.
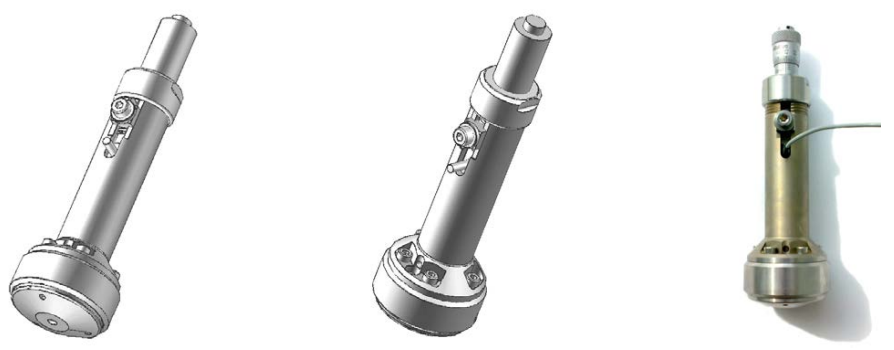

Figure 2.12: Second generation High Viscosity Inkjet.

\subsection{Material supply system}

To study the effect of droplet break-up induced by the printhead, it is essential to have an undisturbed fluid supply. Pressure and flow fluctuations from the fluid supply might give unexpected effects. The required low flow and high pressure do not result in a standard pumping solution. The requirements for the fluid supply are:

$\diamond$ Broad spectrum of materials processable. Ability to process higher viscous materials $(500 \mathrm{mPa} / \mathrm{s})$.

$\diamond$ Low material flow (1 to $5 \mathrm{ml} / \mathrm{min}$ ).

$\diamond$ High pressure (up to $200 \mathrm{bar}$ ).

$\diamond$ Low pressure pulse.

$\diamond$ Chemical resistant.

$\diamond$ Operating time long enough to do experiments.

$\diamond$ Temperature resistant.

$\diamond$ Long lifetime.

$\diamond$ Continuous operation.

$\diamond$ Easy start-up.

$\diamond$ Safe operation. 
In the following sections two types of fluid supplies will be described. First a pressure controlled system, second a version using flow control.

\subsubsection{Pressurized vessel}

The required pressure range combined with the required low fluid flow is relatively rare. Most pumping solutions that can reach the required pressure have a much higher flow rate. But most pumps which do reach the desired flow rate are very limited in achievable pressure. Since most pumping solutions introduce a pressure fluctuation the easiest solution to achieve the requirements is using an ink reservoir that is externally pressurized by means of a gas cylinder. Using a stainless steel high pressure vessel as a reservoir a chemical resistant system capable of withstanding high pressures can be achieved. This however results in a batch wise material feed. If the vessel is empty, the system will stop. To achieve a system that can print for a reasonable amount of time one has to select either a large vessel, or a filling alternative has to be taken into account.

Using a large vessel is not preferred, since as the reservoir empties with ink the amount of pressurizes gas in the vessel increases. If unfortunately a leak might occur the large amount of pressurized gas causes a dangerous situation. It is therefore preferred to keep the reservoir which might be filled with gas as small as possible. In figure 2.13 an overview of a gas pressurized single reservoir setup is shown.

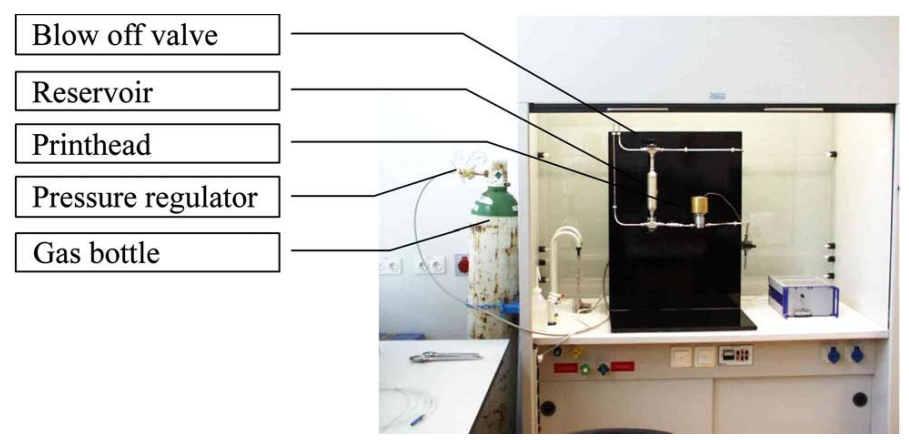

Figure 2.13: Overview of pressurized single reservoir setup.

As mentioned before a small reservoir will empty relatively fast limiting the time to perform experiments. Therefore two parallel vessels might be used which can be alternatively emptied and refilled enabling continuous printing with minimal pressure fluctuations as described in WO2004018212 [17] (figure 2.14).

Although pressurizing the liquid using gas pressure seems a feasible solution, the start-up of such a system is not straight forward. The viscous fluids commonly used 


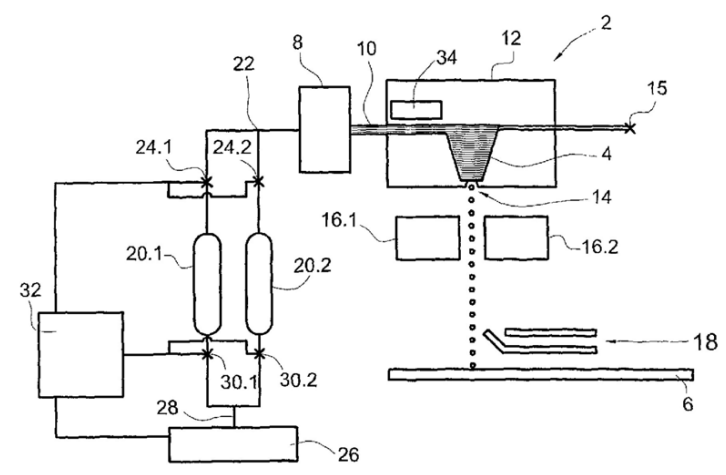

Figure 2.14: Two parallel reservoirs enabling continuous printing taken from WO2004018212 [17]. The material is supplied to the printhead (12) from a reservoir (8) which is pressurized by high pressure cylinder 20.1 or 20.2. If one of both cylinders is empty a control system (32) is available to switch between the cylinders by opening or closing cocks 24.1 and 24.2. The cylinder can consequently be refilled disconnected from the printing system by means of opening cock 30.1 or 30.2 and pressurizing using pump 26. The figure also displays an electrostatic deflection unit (16.1 and 16.2). Also a recycle gutter for unused droplets is visualized (18) and the printing substrate (6).

demonstrate shear thinning behaviour. This results in a normal operating pressure which is much lower than the necessary start-up pressure. In practice the pressure is increased to the pressure needed to start the jet. When the jet is started it is necessary to quickly depressurize the feed vessel to the normal operating pressure. During this depressurization phase the fluid jet exits the nozzle much faster than is intended, emptying the reservoir very rapidly, or even worse, resulting in atomizing of the jet. To minimize this effect direct user feedback is necessary to immediately depressurize the system when the jet has started. Since the jet start is difficult to predict this procedure can be best performed by hand, and is relatively difficult to automate.

\subsubsection{Constant flow dampened system}

If a more automated start-up is desired, a system which delivers a constant fluid flow is preferred over a system regulating the supply pressure. If a constant fluid flow is supplied to the printhead the pressure will regulate itself, resulting in a pressure peak at start-up which decreases directly thereafter without excess material usage.

Although the number of constant flow pumps is limited, pumps used for HPLC 
purposes are flow controlled and have an operating regime which is ideal for single nozzle printing applications. Although these pumps are designed to have as less pressure fluctuation as possible, the pressure pulse during operation is too large to enable stable jetting.

To create a constant material flow and remove the fluctuation a damper can be used. However fluid dampers for these low flow rates are only efficient in a very narrow pressure range. Dependent on the material and specific nozzle used in the printhead, the resulting operating pressure will change significantly. Since changing of the damper with every change in material or nozzle size is not desirable the system needs to be adjusted to keep the pressure at the damper in its most optimal range during operation. By placing the damper directly after the pump and adding an overpressure valve, the pressure of the damper can be set enabling a pulsation free fluid flow [18] ( figure 2.15). As long as the system is designed that the set pressure of the overpressure valve is higher than the resulting pressure in the printhead, a pulse free fluid supply is created which enables automated start-up and continuous operation.

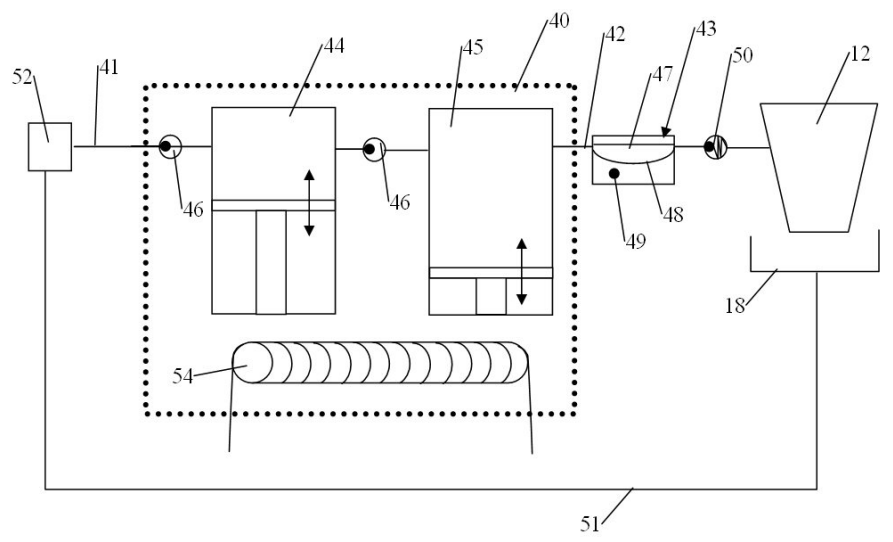

Figure 2.15: Constant flow fluid supply taken from WO2008060149 [18]. Where 40 represents a dual piston pump. The first piston (44) performs the pumping action by means of one way valves (46), and the second piston (45) equalizes the fluid flow to maintain an almost uniform flow. The addition of pulse damper 47 results in a pulse free material supply as long as it is within its working pressure range. Therefore an overpressure valve (50) is added to assure the pulse damper to remain in its effective range at all times. Resulting in a pulse free constant fluid flow supplied to the printhead (12). Unused printed droplets can be collected in gutter 18 and returned by means of the return tube (51) back towards the main fluid reservoir (52). 


\subsection{Droplet visualisation}

To investigate the performance of the developed printhead it is necessary to visualise the droplets generated. In this section an overview is given of available droplet visualisation techniques.

\subsubsection{Stroboscopic illumination combined with a free running or trig- gered camera}

With this principle a stroboscopic light is triggered in exactly the same frequency as the droplet generation frequency (figure 2.16). The camera visualises an image of hundreds of droplets on top of each other since the shutter time of the camera is an order of magnitude lower than the droplet generation rate. If the droplets are generated stably, this results in a very sharp image, but if the droplets show more instability, this type of imaging only shows a blurry image and a different imaging technique is necessary.

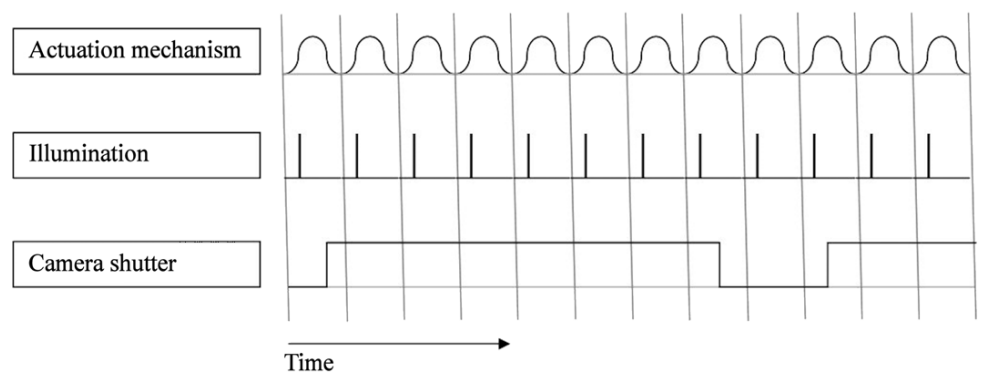

Figure 2.16: Synchronisation of strobe with actuation mechanism, free running camera.

Using only one single strobe flash per camera image enables the visualisation of single droplets (figure 2.17). To be able to compare the different images the strobe is still synchronized with the actuation mechanism. The strobe light should be able to deliver enough light during this single flash to project the image resulting in higher demands for the strobe. With this specific imaging technique it is possible to visualise single droplets in flight enabling the visualisation of less stable drop formation processed. However due to the relatively low repetition time of the camera only several images can be captured per second. Thus only a selection of the generated droplets can be seen.

For the single shot strobe as well as for the continuous strobe system it is necessary to use a strobe flash which is short enough. If for instance the droplets travel at a 


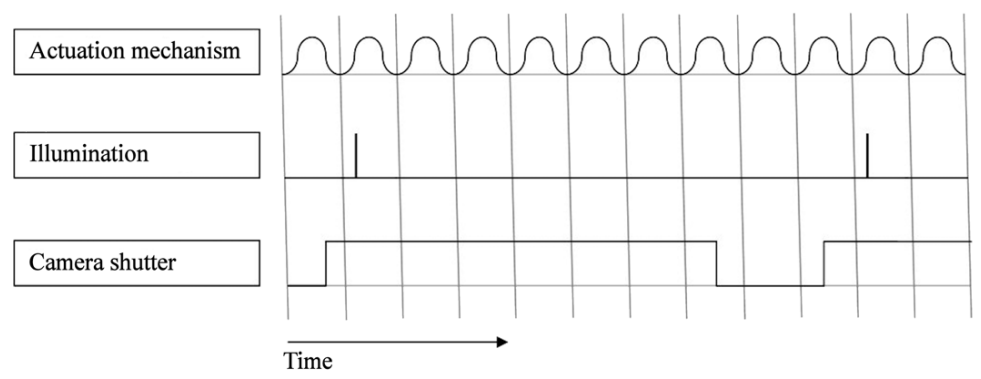

Figure 2.17: Synchronisation of strobe with actuation mechanism, triggered camera.

speed of $20 \mathrm{~m} / \mathrm{s}$ and a flash duration of $250 \mathrm{~ns}$ is used, the droplet will travel $5 \mu \mathrm{m}$ during the flash. Depending on the expected droplet speed and required resolution, a maximum allowable flash duration should be chosen. There are several ways of generating a flash short enough for creating images of high speed droplets:

$\diamond$ LED strobe: typical $300 \mathrm{~ns}$, minimal $100 \mathrm{~ns}$ [47].

$\diamond$ Spark strobe: typical $11 \mathrm{~ns}$, minimal $8 \mathrm{~ns}$ [48].

$\diamond$ Laser source with fluorescent: typical $6 \mathrm{~ns}$, minimal $70 \mathrm{ps}$ [49].

\subsubsection{Static illumination combined with a high speed camera}

To visualise dynamic effects of one specific droplet, it is necessary to shift towards high speed imaging. Although one single droplet can be followed exactly, the duration is relatively limited due to the limitation in maximum captureable frames (figure 2.18).

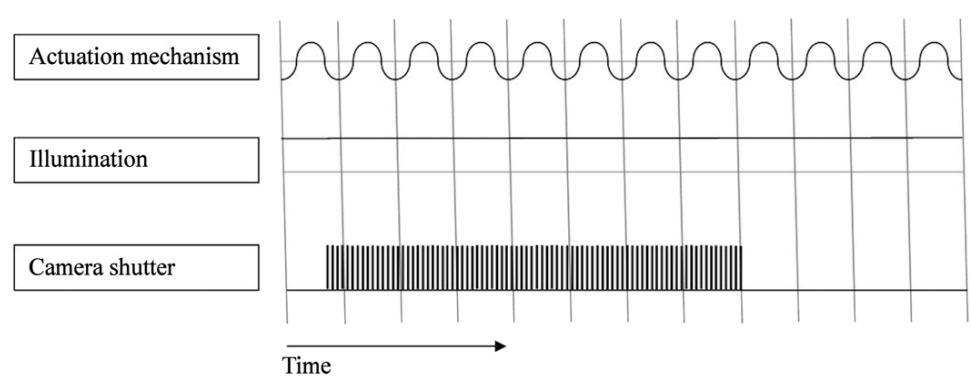

Figure 2.18: High speed camera, no synchronization required, however limited in duration. 
The main goal of this chapter lies in developing an inkjet printhead capable of processing higher viscous materials compared to conventional printheads. So primary only visualizing the droplet creation is sufficient to prove the functionality of the printhead. The most basic principle of using a free running camera together with stroboscopic LED imaging will be sufficient. In the next chapter also a triggered camera is used to achieve single drop visualisation.

\subsection{Experimental validation}

Over the past ten years a lot of experimental work has been performed using the printheads described. In this thesis two examples are given. As a reference the experiments are first performed using a low viscous material (2-propanol) to achieve insight in the operating window. Secondly a high viscosity test oil of $200 \mathrm{mPa} \cdot \mathrm{s}$ is used and the process window is attained.

Figure 2.19 shows the experimental results obtained processing 2-propanol related to the predicted operating window as described in paragraph 2.1.2. A spark eroded stainless steel nozzle with a diameter of $50 \mu \mathrm{m}$ is used; $300 \mu \mathrm{m}$ in length. The printhead is equipped with a vibrating pen with a diameter of $3.3 \mathrm{~mm}$, positioned at a distance of $50 \mu \mathrm{m}$ from the nozzle. The measurement points in the graph represent the lower and upper frequency ranges at fixed velocities, measured with different piezo amplitudes to show the maximum operating window. With respect to jet velocity the measurements were stopped when the break-up point moved beyond $12 \mathrm{~mm}$ to keep within a common range for electrostatic charging and deflection of droplets. Alternative deflection methods can be envisioned as described in more detail in chapter 3.

Taking a closer look at the experimental results one can see the lower speed limit to be higher than predicted. The nozzle used during the experiments was a stainless steel nozzle plate compared to the prediction curves which are based on small glass capillaries. A nozzle plate construction gives more opportunity for the fluid to spread requiring a higher speed to form a jet. The upper velocity limit is not reached during the experiments since the break-up point shifted beyond the set limit of $12 \mathrm{~mm}$.

With respect to the frequency boundaries, the lower frequency boundary looks similar as predicted. The upper frequency boundary seems to be limited in maximum working range. This can be expected due to the relatively large piezo element used, designed for high viscous fluids capable of high forces, although limited in operating frequency due to its relatively high capacity.

Figure 2.20 shows the experimental results obtained processing a $200 \mathrm{mPa} \cdot \mathrm{s}$ test oil related to the predicted operating window. 


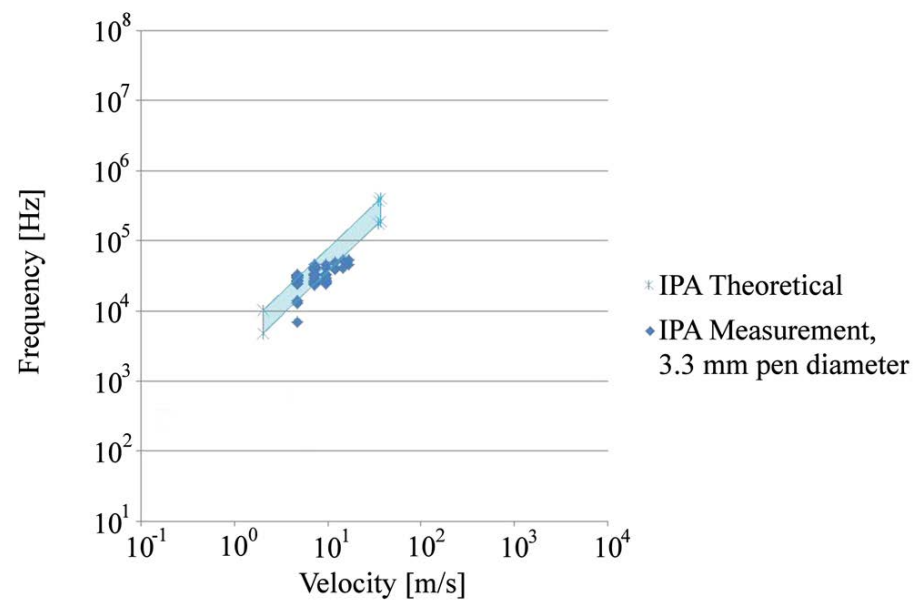

Figure 2.19: Measurements of 2-propanol related to theoretical boundaries.

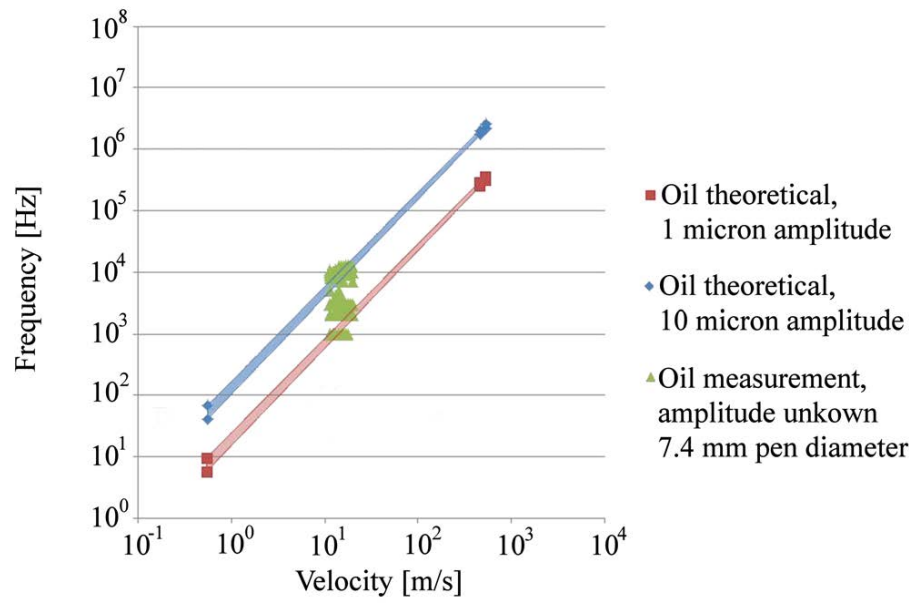

Figure 2.20: Measurements of $200 \mathrm{mPa} \cdot \mathrm{s}$ test oil related to theoretical boundaries. Two theoretical operating windows are shown, one using a $1 \mu \mathrm{m}$ vibration amplitude, one using a $10 \mu \mathrm{m}$ vibration amplitude.

A spark eroded stainless steel nozzle with a diameter of $80 \mu \mathrm{m}$ is used; $300 \mu \mathrm{m}$ in length. During these measurements the printhead is equipped with a vibrating pen with a diameter of $7.4 \mathrm{~mm}$, positioned at a distance of $50 \mu \mathrm{m}$ from the nozzle to obtain jet break-up near the nozzle. Here the measurement points in the graph also represent the lower and upper frequency ranges at fixed velocities, measured with 
different piezo amplitudes to show the maximum operating window. The diameter of the vibrating pen was increased to $7.4 \mathrm{~mm}$ diameter to achieve break-up within the $12 \mathrm{~mm}$ range as described above. The measurements seem to differ significantly from the predicted range, but one has to keep in mind the predicted range only represents one single amplitude setting. Therefore two prediction curves are displayed; one using an amplitude of $1 \mu \mathrm{m}$, the other an amplitude of $10 \mu \mathrm{m}$. An increase in amplitude leads to a shift into a higher frequency domain (blue in figure 2.20), analogue a decrease in amplitude results in lower achievable frequencies (red in figure 2.20). The experimental results show this effect; depending on the actuating voltage the frequency domain can be adjusted. Also here the difference in lower and upper speed limit can be explained due to the different nozzle geometry used and the maximum measured break-up length of $12 \mathrm{~mm}$.

\subsection{Discussion}

The experiments show the capability of the printhead to process higher viscous fluids as well as the ability to create droplets from low viscous materials. The operating window of the system with low viscous materials is in line with the theoretical curves based on correlations found in literature. With high viscous fluids the uncertainty of which amplitude one can expect results in a less straight forward correspondence with the prediction curves. One can however derive that if a longitudinal vibration of the nozzle had been used, similar as used in the experiments of Sakai et al. for the determination of the prediction curves described in paragraph 2.1.2, the effect of the vibrating member close to the nozzle correlates with an amplitude of $10 \mu \mathrm{m}$. A longitudinal displacement of the nozzle (figure 2.21) is linked directly to a jet speed variation from which the resulting flow fluctuation can be calculated.

The model described in paragraph 2.1.2 gives an approximation of the flow fluctuation of the system developed depending on the amplitude of the vibrating member. This way an approximation can be made between the differences in amplitudes necessary in both systems to achieve equal drop formation. If a constant outflow from the nozzle is assumed during the longitudinal agitation, a $10 \mu \mathrm{m}$ amplitude with an actuation frequency of $10 \mathrm{kHz}$ would lead to a flow fluctuation directly past the nozzle of $15.1 \%$. To achieve a similar flow fluctuation using an actuated pen close to the outflow opening as used in the developed printhead, one can calculate with equation 2.18 that an amplitude of only $120 \mathrm{~nm}$ is necessary, a reduction with a factor 80. Since achieving high amplitudes at high operating frequencies is not straight forward, the significant reduction in necessary amplitude gives a good impression of the higher performance of the printhead compared to commercial available systems. 
Longitudinal vibrating nozzle: Experiments Sakai et al.
Vibrating pen close to nozzle: Experiments described in this thesis
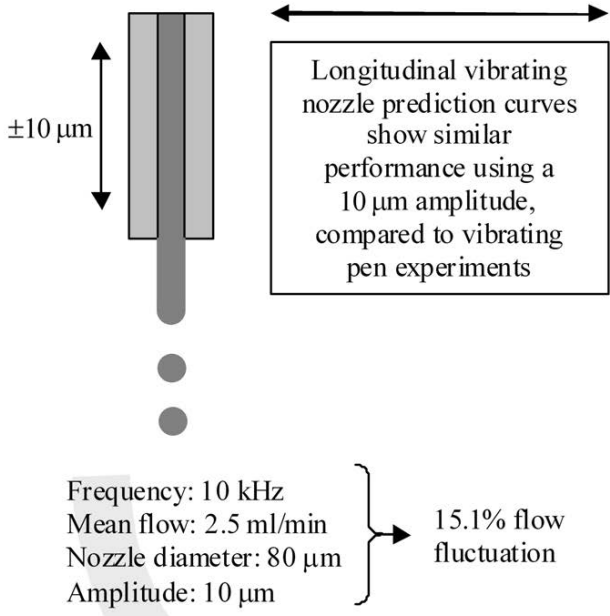

Based on equation 2.18:

Frequency: $10 \mathrm{kHz}$

Mean flow: $2.5 \mathrm{ml} / \mathrm{min}$

Vibrating pen diameter: $7.4 \mathrm{~mm}$

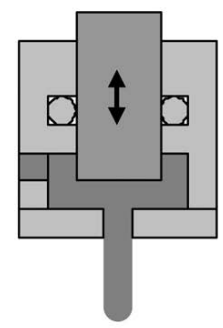

$120 \mathrm{~nm}$ pen amplitude

Figure 2.21: From theoretical prediction curves to an estimation for the amplitude used.

\subsection{Alternative designs}

In the previous paragraphs the development and validation of a printhead is described to process high viscous fluids. Depending on the specific application of the printhead several alternative designs might be of interest. In the following subsections some alternative designs or upgrades will be described. 


\subsubsection{Vibrating nozzle}

One of the main elements in the design of the printhead is the small distance between the vibrating element and the nozzle plate. For the first design it was logical to vibrate the focusing member above the outflow opening. However the same effect can be achieved by correct dimensioning of the inlet channel and vibrating the nozzle plate. With this design not only a pressure fluctuation is generated inside the printhead, but also the generated jet is subjected to an extra exit speed difference, adding extra efficiency to the system [20] (figure 2.22).
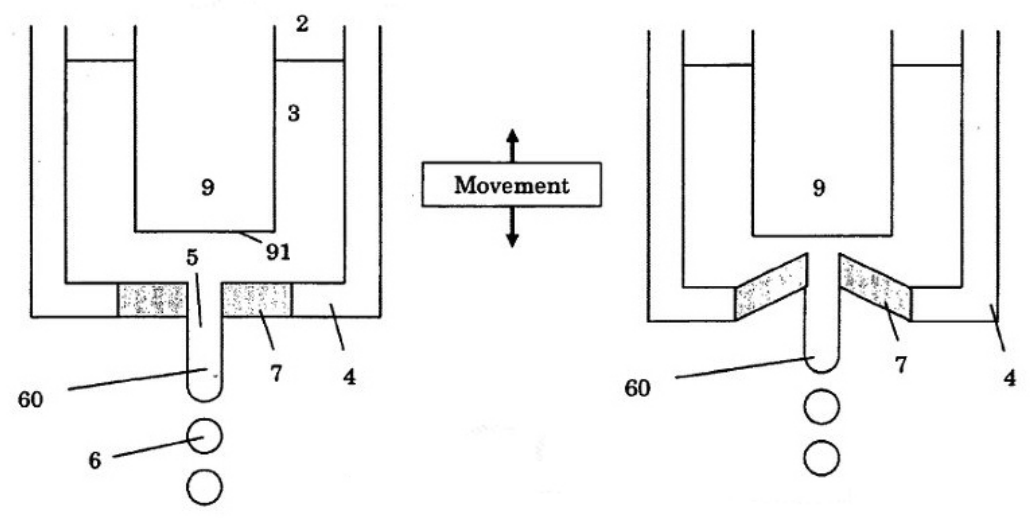

Figure 2.22: Vibrating nozzle arrangement taken from WO2009061202 [20]. Material 3 is processed through nozzle hole 5 to generate a jet (60) breaking up into droplets (6). The jet is disturbed by a piezo element integrated in the nozzle (7). To achieve an effective perturbation a focussing member (9) is positioned close to the nozzle.

\subsubsection{Mechanical droplet break-up}

Usage of a piezo element is ideal due to its flexibility in frequency range and amplitude for experimental purposes. For industrialisation however, usage of a piezo element as actuator has quite some drawbacks. To operate it, one needs a lot of electronics, from a frequency generator to a piezo amplifier. Also the element itself is quite fragile. For industrialisation in rough environments it would be beneficial if another actuation mechanism was available to create droplet break-up with the desired materials. The main challenge is the required actuation frequency in the order 
of kHz. Almost no mechanical actuators are available operating at these frequency ranges. Therefore it is desirable to incorporate some sort of multiplier, which can convert a low frequency mechanical vibration into the desired vibration in the $\mathrm{kHz}$ range.

The piezo element causes a vibrating member to move perpendicular to the nozzle opening. This results in speed differences in the ejected fluid jet leading to jet breakup. In WO2009028947 [19] a different approach is taken. Not moving the element perpendicular but moving a notch parallel to the outflow opening. This results in a different flow resistance towards the nozzle leading to speed differences as well. This movement might as well be in a circular direction enabling the placement of multiple notches on a disk. The disc with multiple notches acts as a multiplier to convert one single rotation in a multitude of agitations enabling $\mathrm{kHz}$ vibrations to be introduced (figure 2.23).
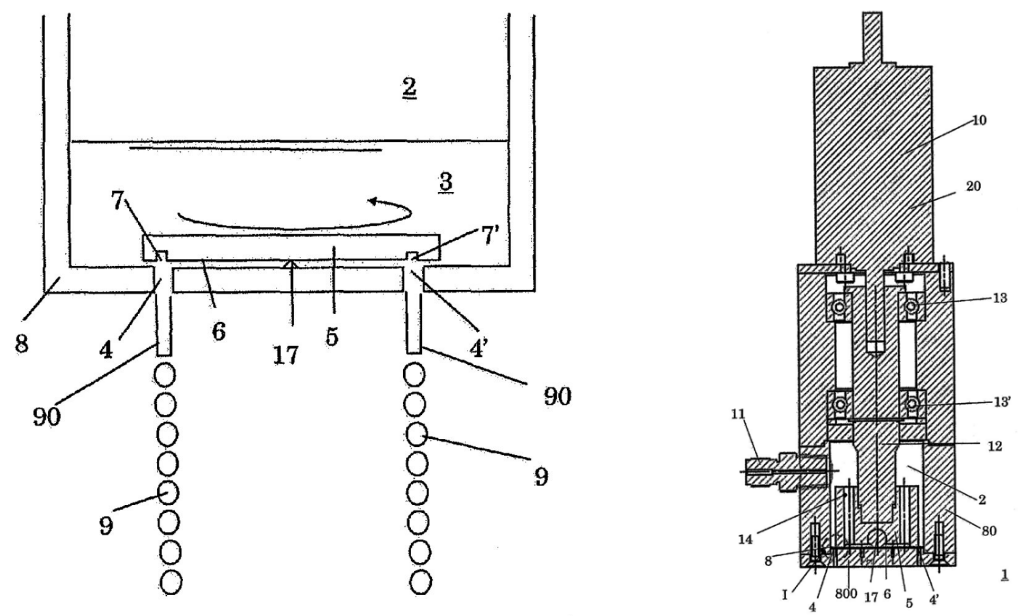

Figure 2.23: Mechanical droplet break-up taken from WO2009028947 [19]. Left a general representation is shown where material 3 is pressurized out of outflow openings 4 and 4' by means of a pressurized gas (2). The resulting jets (90) break-up into droplets (9) as a result of a vibration introduced by indents 7 and 7' in disc 5 rotating closely above the nozzle holes. Right a cross-section is shown of this concept. Here also the rotating disk 5 is shown and nozzle holes 4 and 4'. The disc is rotated by means of an electric motor (10), a driving axle (12) which rotates freely in bearings (13). The material is fed into the printhead through connection 11. A further in-depth description of all noted numbers can be found in the patent application. 
Although it can be argued that perpendicular agitation might be more efficient, the robust layout of a rotating angular disk might be preferable in rough industrial environments. Especially since circular multi nozzle droplet generators become interesting in specific non printing applications (chapter 4) in the field of uniform particle generation. In these applications also the opposite layout can be envisioned where the nozzle plate rotates underneath a stationary notched surface [21] (figure 2.24).
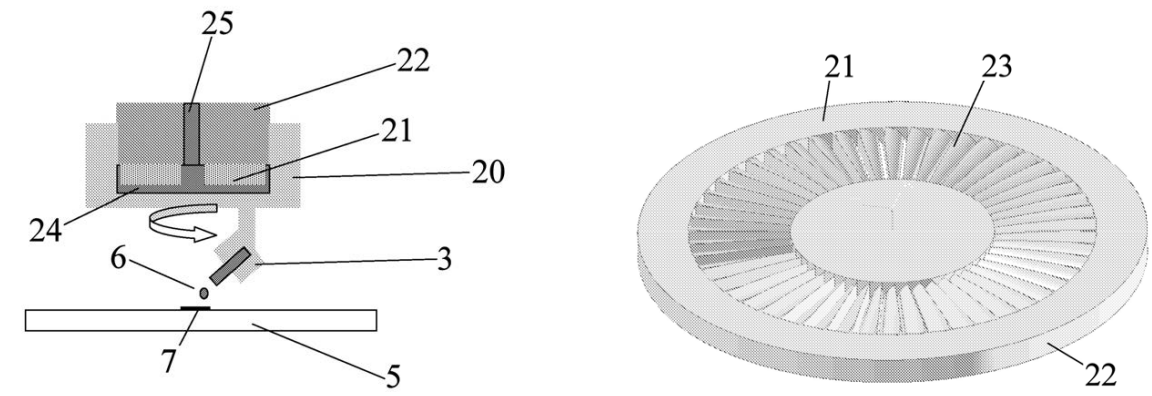

Figure 2.24: Rotating nozzle plate in mechanical droplet break-up taken from WO2010068108 [21]. Left a general overview is given of the system where the material is fed through supply channel 25 . Here the indents (21) are stationary fixed on mounting base 22 . The nozzle (3) is attached on a rotating element (20). The principle is similar to figure 2.23 however now the nozzles are rotating. The right figure displays a possible layout of the indents (23) causing the pressure fluctuation. A further in-depth description of all noted numbers can be found in the patent application.

\subsubsection{Pressure independent droplet generation}

When processing highly viscous material the necessary static pressure to eject the material increases significantly. When using a piezo actuated system as described in paragraph 2.1.1. part of the energy of the piezo element is used to push against this static pressure. When operating a single nozzle, the vibrating element has a relatively small surface area subjected to this static force. In the design of a multi nozzle system the required actuation surface increases. If basic scaling is used the static force on the piezo element increases drastically resulting in poor piezo efficiency. Only increasing the area inside the fluid is a more efficient solution. In WO2009151332 [22] a pressure independent layout is sketched optimizing piezo performance (figure 2.25). Several different layouts can be generated even enabling central mounting of the nozzle plate (figure 2.26). 


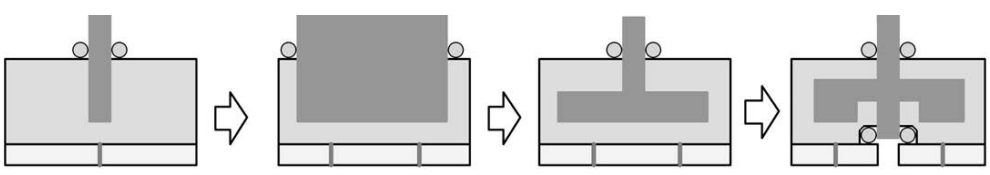

Figure 2.25: From single nozzle towards multi nozzle pressure independent.
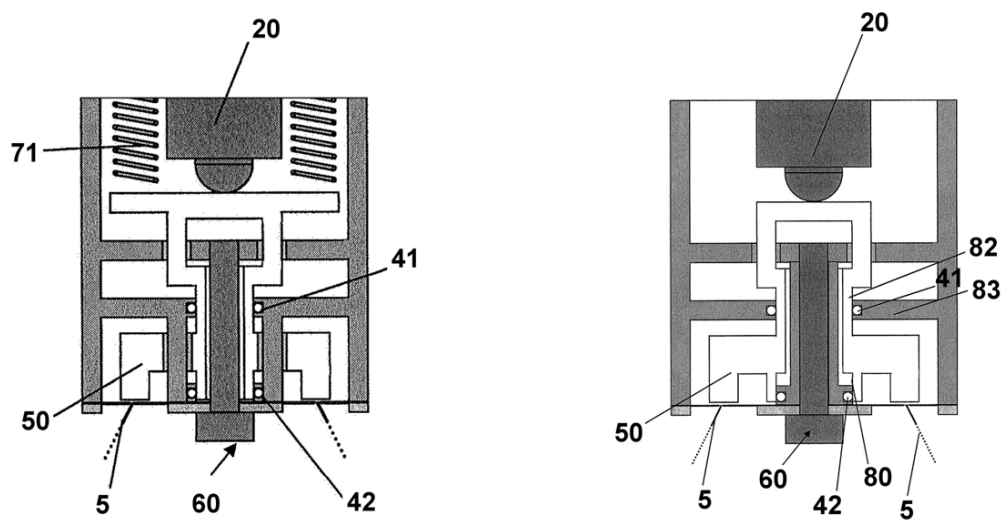

Figure 2.26: Different pressure independent designs using central nozzle plate attachment taken from WO2009151332 [22]. Left the piezo element (20) is held under pretension by means of springs (71). The piezo presses against vibrating member 50 designed so the cross-sections of seals 41 and 42 result in a pressure neutral mounting of the vibrating member. The assembly is mounted by a central mounting bolt (60) and the fluid is ejected out of the nozzle plate as jets (5). The jets leave the nozzle surface under an angle since the nozzle holes are laser-drilled especially under this angle to achieve a conical spray pattern. The right figure displays a simplified construction where the vibrating member (50) can be manufactured more easily and fewer parts are necessary compared to the left figure. A further in-depth description of all noted numbers can be found in the patent application.

\subsection{Concluding remarks}

A printhead is developed capable of printing a much broader range of materials compared to commercial available systems, shifting the maximum viscosity boundary from $20 \mathrm{mPa} \cdot \mathrm{s}$ towards $500 \mathrm{mPa} \cdot \mathrm{s}$ at jetting temperature. Based on continuous inkjet technology a piezo driven single nozzle printhead is developed using a vibrating pin 
close to the nozzle to supply the pressure fluctuation as close to the nozzle as possible in order to enable droplet break-up in the generated high viscous jets. The vibrating system is sealed using o-ring seals to allow high printing pressures. The general printhead layout is described in an analytical model to predict the systems behaviour depending on specific design dimensions resulting in the final design. To fully test the printhead a material feeding mechanism is developed capable of delivering a continuous flow at the required high pressures. A setup is realised utilizing stroboscopic illumination to study droplet break-up.

From experiments it can be concluded that the design utilizing a piezo driven actuator pin close the nozzle has proven to be a good starting point for most applications. The systems performance can be compared to a longitudinal vibrating nozzle but only $1.2 \%$ of the amplitude is needed with the final design, giving an indication of the extended performance of the system. The layout is easily adaptable if required, and it has shown to be robust in practice. A key aspect of the current design is its ability to process a wide range of fluids within a wide range of operating frequencies. One should however keep in mind that this flexibility comes at a price. The chosen piezo element is large in order to be able to cope with this wide variety of loads and frequencies. If the system would be industrialized, it can be optimized to run with one single material at a fixed frequency. Using resonance frequencies in stead of avoiding them could lead to a system with an even higher achievable viscosity, unfortunately with a very limited operating window. When developing a dedicated printhead the distance between the vibrating member and the nozzle can be fixed, so the membrane solution becomes an interesting option again. Depending on the specific application of the printhead alternative actuation designs might be of interest as described in the previous paragraph.

But this flexibility in the system is a characteristic that is key in the step from graphics towards industrial printing. It allows the ink/material to be optimized for the final purpose of the product instead of optimization of the formulation to be able to process it by means of inkjet. Instead of developing a material which can be printed and luckily still has some of the function it should have when deposited, a material with an optimal function is developed and the deposition system follows. Eventually when the process is proven and the material is finished, the printhead can be redesigned to work most efficient with this specific material at the required operating point as described above. 


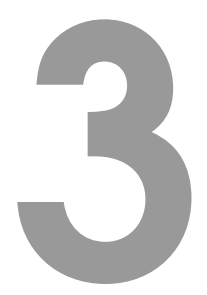

\section{Droplet selection ${ }^{1}$}

In the previous chapter a continuous stream of small high viscous droplets is created, in this chapter droplet selection will be discussed. Three selection mechanisms are described, electrostatic deflection, ballistic deflection and airjet manipulation.

Electrostatic deflection requires the processed materials to be conductive, to allow droplet charging. A solution is described for minimizing the amount of material adaptation to enable the use of electrostatic deflection. If no material adjustment is allowed two alternatives are discussed. Ballistic droplet deflection utilizes a second inkjet system as a selection mechanism. The droplets of the second inkjet are set to collide with the droplets generated by the continuous printhead to divert them from their path. Alternatively an air-jet selection mechanism is described. This mechanism uses a continuous air-jet which is selectively directed towards the droplet steam. By selecting a small jet in combination with the development of a fast air-jet movement mechanism a system is fabricated capable of single droplet selection.

\footnotetext{
${ }^{1}$ The patents on which this chapter is based are filed as:

$\diamond$ J.J. Michels, R.J. Houben, "Curable compositions for continuous inkjet printing and methods for using these compositions", (2007), EP1869133.

$\diamond$ R.J.J. Boot, R.J. Houben, G. Oosterhuis, A.P. Aulbers, “Droplet selection mechanism”, (2009), WO2009061201.

$\diamond$ R.J.J. Boot, R.J. Houben, G. Oosterhuis, A.P. Aulbers, "Droplet selection mechanism”, (2009), WO2009061195.
} 


\subsection{From electrostatic deflection towards alternatives}

Using continuous inkjet technology to create droplets, results in a continuous stream of droplets being formed. To enable selective droplet deposition in space and time on a substrate a droplet selection mechanism is required. Commercially available continuous inkjet systems use electrostatic droplet deflection mechanisms, in which the droplets are selectively charged (figure 3.1) and deflected by means of an electrostatic field. If a binary deflection system is used, a charged droplet is deflected towards a recycle gutter and non charged droplets are deposited on the substrate (illustrated in figure 3.2).

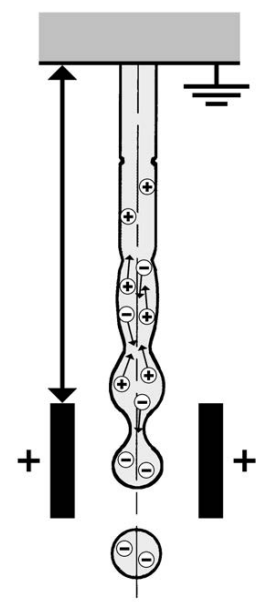

Figure 3.1: Continuous inkjet, droplet charging. An electrical field is applied between the charging electrodes ( + ) and the printhead (grounded). This results in the movement of charges in the liquid jet. Resulting in a negative charged fluid stream just before break-up of the droplets. When break-up occurs this prevents further charge movement towards the droplets resulting in a charged droplet. By selectively applying this electrical field each droplet can be charged selectively.

The charging principle of the droplets requires the used printing ink to be conductive. A minimum of $200 \mu \mathrm{S} / \mathrm{cm}$ conductivity is needed for binary deflection, although higher conductivity levels are preferred up to $2 \mathrm{mS} / \mathrm{cm}$ for multiple deflection systems [50]. Commonly used continuous inkjet systems use low viscous fast drying inks containing high weight percentages (wt\%) of solvents $(80-90 \mathrm{wt} \%)$. The presence of these solvents allows these inks to be made conductive by adding small amounts of salts (up to $5 \mathrm{wt} \%$ ). The ability to use higher viscous materials opens 


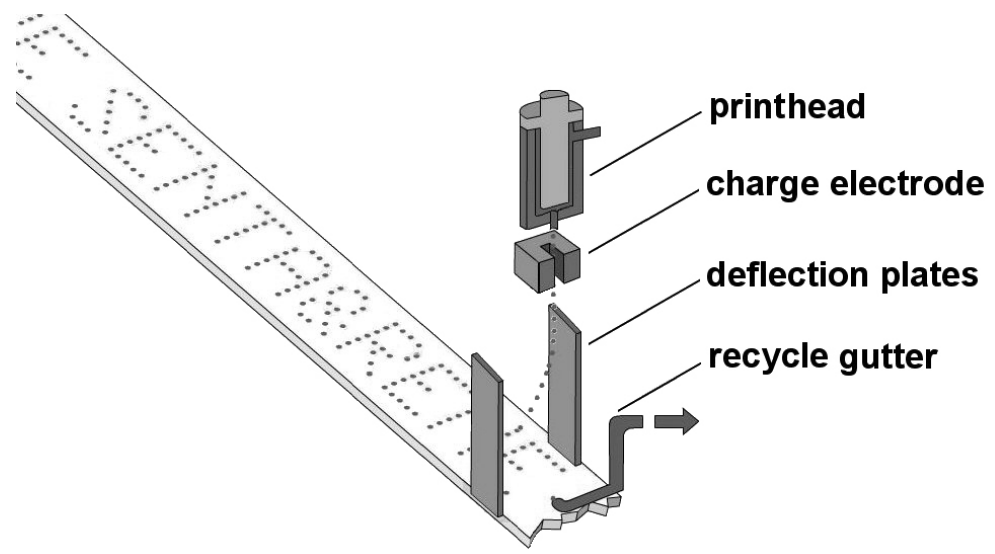

Figure 3.2: Continuous inkjet, deflection. The selectively charged droplets are propelled through a second electrical field, perpendicular to the jet. Charged droplets are influenced by the field resulting in a deviation from their initial path. This deviation can be used to select unwanted droplets for recycling, or direct droplets towards a specific position on the substrate dependent on their charge level.

opportunities to use solvent free inks. Making these inks suitable for electrostatic deflection becomes less straight forward. Due to the absence of solvent the required amount of salt to achieve the same level of conductivity increases significantly. In EP1869133 [23] a method is described how the conductivity can be raised by adding small amounts of water in combination with small amounts of salt. The addition of water causes a small viscosity decrease which results in a higher conductivity, but the main increase in conductivity is related to the high dielectric constant of the added water. This allows the ions of the added salt to move more freely through the liquid, raising the conductivity. In table 3.1 two formulations are described, the first using water as conductivity enhancer $\left(\varepsilon_{\text {rwater }}=80\right)$. In the second formulation the water has been replaced with isopropyl alcohol (IPA) to maintain the same viscosity, however, IPA has a lower dielectric constant $\left(\varepsilon_{r I P A}=18\right)$ showing the decreasing effect on the conductivity. Unfortunately this still requires the material to be adjusted, with possible unwanted side effects on the ink performance. If for instance the droplet generator is used for generating 3D bone implants one can imagine that adding lithium salt to an implant results in an undesired situation (lithium is used as an antidepressant in medicine). Although EP1869133 lowers the required amounts of salt significantly, the development of a selection mechanism that requires no material adaptation is wanted for these applications. 
Table 3.1: The effect of water vs isopropyl alcohol on the conductivity.

\begin{tabular}{|l|l|r|r|}
\hline \multicolumn{2}{|l|}{} & Formulation 1 & Formulation 2 \\
\hline Material name: & Abbreviation: & $\mathrm{wt} \%$ & $\mathrm{wt} \%$ \\
\hline $\begin{array}{l}\text { Triethylene glycol } \\
\text { dimethacrylate }\end{array}$ & TEGDMA & $2.8 \mathrm{wt} \%$ & $2.8 \mathrm{wt} \%$ \\
\hline $\begin{array}{l}\text { Bisphenol A glyc- } \\
\text { erolaat dimethacry- } \\
\text { laat }\end{array}$ & BisGMA & $42.6 \mathrm{wt} \%$ & $42.6 \mathrm{wt} \%$ \\
\hline $\begin{array}{l}\text { 2-Hydroxyethyl } \\
\text { methacrylate }\end{array}$ & HEMA & $42.6 \mathrm{wt} \%$ & $42.6 \mathrm{wt} \%$ \\
\hline Water & $\mathrm{H}_{2} \mathrm{O}$ & $7.5 \mathrm{wt} \%$ & $0 \mathrm{wt} \%$ \\
\hline Isopropyl alcohol & $\mathrm{IPA}^{2}$ & $0 \mathrm{wt} \%$ & $7.5 \mathrm{wt} \%$ \\
\hline Lithium triflate & $\mathrm{LiCF}_{3} \mathrm{SO}_{3}$ & $4.4 \mathrm{wt} \%$ & $4.4 \mathrm{wt} \%$ \\
\hline Irgacure 891 & Irgacure 891 & $1.0 \mathrm{wt} \%$ & $1.0 \mathrm{wt} \%$ \\
\hline Viscosity [Pa·s] & $104 \cdot 10^{-3}$ & $132 \cdot 10^{-3}$ \\
\hline \multicolumn{2}{|l|}{ Conductivity $[\mu \mathrm{S} / \mathrm{cm}]$} & 270 & 46 \\
\hline
\end{tabular}

\subsubsection{Selection of alternative droplet selection methods}

If no material adjustment is allowed an alternative deflection mechanism has to be developed. The requirements for the deflection system can be summarised as:

$\diamond$ Single droplet selection.

$\diamond$ Functional up to $20 \mathrm{kHz}$ droplet generation rates.

$\diamond$ Operational with non conductive material.

$\diamond$ No material adaptation.

$\diamond$ Minimum of $2^{\circ}$ deviation for selection.

$\diamond$ Economically feasible.

$\diamond$ Preferably no contamination of recycled ink.

Single droplet selection implies that the selection mechanism needs to be able to alter the flight path of each individual droplet. Electrostatic deflection uses the ability to selectively charge each droplet, thus altering specific characteristics of the individual droplets (electric charge). If no use can be made of specific properties of the material, one has to resort to different methods to influence the droplets path. Next a selection of the most promising deflection methods is given:

Using air to push the droplet

1. Micro air-jet deflection using a switchable micro air-jet: an air-jet is aimed at 
the initial droplet trajectory. By selectively switching the air-jet on and off, droplets can be deflected.

2. Micro jet deflection using a movable micro air-jet: instead of switching the airflow, a continuous air-jet is used. The air-jet is selectively moved into the droplets path, deflecting the passing droplet.

3. Micro jet deflection using the "Coanda-effect" (using the effect that a fluid or gas flow prefers to follow the curvature of a nearby convex surface rather than passing in its initial straight path): similar to the previous method, only instead of moving the origin of the air-jet, the air-jet is deflected towards the droplet trajectory by slightly touching the jet with an object.

Using another liquid to push the droplet

4. Ballistic deflection: using a different jetting device to "shoot" droplets out of their path with different droplets.

Using another rigid material to "push" the droplet

5. Mechanical droplet selection: using a fast moving member selectively touching the passing droplets resulting in the droplets being captured by the moving member.

Using the droplet itself

6. Laser ablation: by aiming a pulsed laser on the droplet, part of a droplet evaporates, delivering a shock-wave propelling the droplet out of its initial path.

From a research point of view, all options are interesting. However, an economically feasible solution needs to be found, eliminating the usage of relatively expensive laser solutions (option 6). It can be doubted whether an air-jet can be switched on and off with the required frequencies (option 1). And also mechanically moving a selecting member (option 5), an air-jet (option 2) or even a small air-deflector (option 3 ) at these frequencies is not straight forward. Although ballistic deflection (option 4) might lead to contamination of the recycled ink, it seems a solution in which the selection frequency is not limiting. Therefore, ballistic deflections will be investigated in the following paragraph. Using a complex second inkjet system to enable droplet deflection for the continuous inkjet seems to be able to deliver the required result. However, operating two printheads, aligning their trajectories and timing their droplets collision can be done in the lab, industrialization will be difficult. Alternatives involve mechanical movement. The ability to mechanically move an object at 
high frequencies is a balance between available power and weight. The lower the weight the easier it will become to achieve the required frequencies. So it is chosen to investigate the movable air-jet as well.

\subsection{Ballistic deflection, droplet selection by collision}

In patent literature selection mechanisms based on colliding droplets can be found. In US 4341310 such a selection mechanism is described where two continuous inkjet systems are used; one using a polar fluid enabling electrostatic deflection, the other a non polar fluid for which a different selection mechanism is required. By synchronizing both droplet generators, it becomes possible to shoot non-polar droplets out of their initial path using the other selective droplet generator (figure 3.3). The non-effected droplets of the non-polar fluid continue undisturbed (vertical moving droplets), the collided droplets proceed under a deviated angle, the continuously generated polar droplets not used for collision are aimed to pass the polar droplets and continue horizontally.

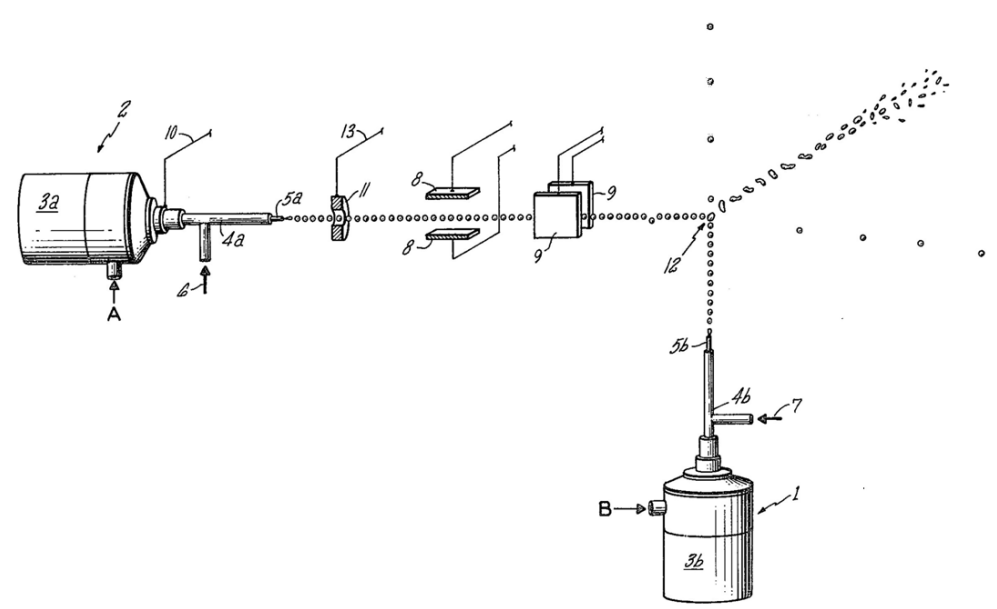

Figure 3.3: Ballistically controlled non-polar droplet dispensing method and apparatus [51]. A printhead (3b) jets a continuous stream of droplets out of a nozzle (5b). Another printhead ( $3 a$ ) is equipped with a deflection system consisting a charge electrode (11) and deflection plates 8 and 9 allowing the droplets to be directed past the droplet stream of printhead $3 \mathrm{~b}$ or to collide with the droplet stream at position 12 causing droplets to be diverted from their initial path. A further in-depth description of all noted numbers can be found in the patent application. 
When using two relatively identical systems one can envision that the collision will have sufficient effect to result in a substantial deflection. In WO 2009061201 [24] a system is described utilizing the high viscosity droplet generator as developed in the previous chapter but using a drop-on-demand system for droplet selection (figure 3.4).

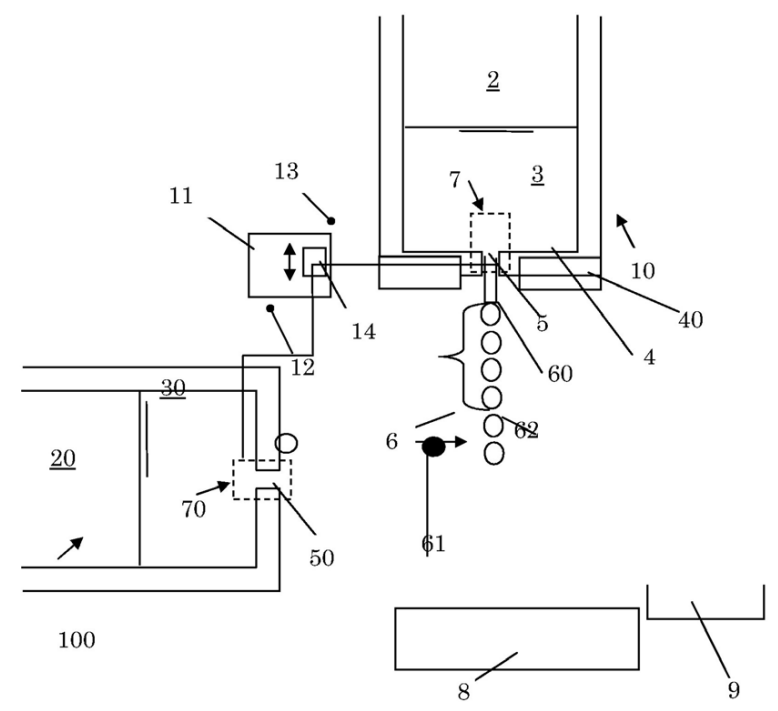

Figure 3.4: Droplet selection mechanism [24]. A printhead (2) ejects a continuous stream of droplets out of a nozzle (5). Another printhead (20) is arranged to selectively generate droplets out of nozzle (50) and is aimed at the droplets stream emerging from printhead 2. An electronic timing device (11) is used to trigger printhead 20 to collide with a predetermined droplet from printhead 2 creating a droplet selection mechanism. Collided droplets are collected in gutter 9 while non collided droplets reach substrate 8 . A further in-depth description of all noted numbers can be found in the patent application.

In this case the difference between both colliding droplets is much larger. Not only are the drop-on-demand droplets smaller than the droplets from the continuous inkjet, but their speed is also significantly lower $\left(55 \cdot 10^{-6} \mathrm{~m}\right.$ diameter with a velocity of 1.5 $\mathrm{m} / \mathrm{s}$ for the drop-on-demand printhead compared to $110 \cdot 10^{-6} \mathrm{~m}$ diameter with 10 $\mathrm{m} / \mathrm{s}$ for the continuous inkjet). The question arises whether the required deflection angle can still be achieved.

Another issue which can occur when using ballistic deflection can be seen in figure 3.3 where the collided droplets are combined, mixing both fluid flows, contaminating the return flow. However from literature [52] several different collision 
regimes can be distinguished (figure 3.5), revealing collision regimes without mixing of the droplets.

Coalescence: Collision of two droplets where at the moment both droplets touch each other, the droplets merge into one single droplet (figure 3.5a).

Reflective separation: Collision of two droplets where both droplets coalescence only temporary. The liquid will spread radially, forming a torus-like droplet [53]. The resulting pressure difference of the inner and outer region of the resulting droplet pushes the liquid away from the centre, resulting in a stretching column which might eventually break-up into two or multiple droplets (figure $3.5 b)$.

Stretching separation: Collision of two droplets where at the moment of impact only a small part of the droplets is in direct contact. Due to surface tension these regions try to hold the droplets together, however the main part of the droplet tries to follow its initial trajectory. If the droplets differ in size, the smallest droplet will have a higher internal pressure, resulting in flow towards the larger droplet. The droplets will separate again when the stretching energy is larger than the surface energy. Satellite droplets might be generated during this separation phase (figure $3.5 \mathrm{c}$ ).

Bouncing: If two droplets approach each other a layer of the surrounding medium gets trapped in between. When the droplets move closer the pressure will rise in this layer. However if the relative velocity of both droplets isn't large enough to overcome this pressure build-up, bouncing will occur and the droplets will not mix (figure 3.5d).

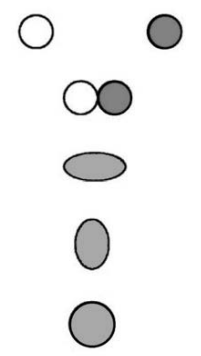

(a) Coalescence

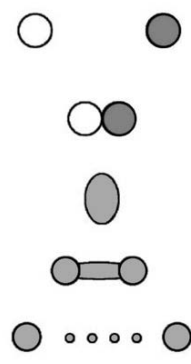

(b) Reflective separation

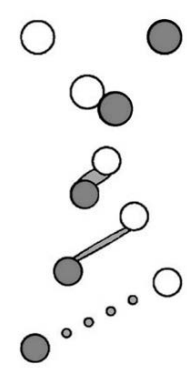

(c) Stretching separation

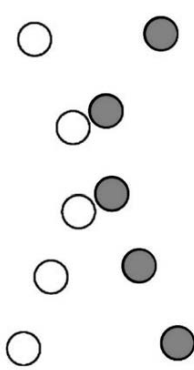

(d) Bouncing

Figure 3.5: Outcome regimes of droplet collisions [52]. 
To achieve insight in which regime can be expected many authors use a combination of the Weber number $(W e)$ and the impact parameter $(I)$.

$$
\begin{gathered}
W e=\frac{\rho \cdot d_{s} \cdot V_{r}^{2}}{\sigma} \\
I=\frac{2 \cdot \delta}{d_{l}+d_{s}}
\end{gathered}
$$

Here $\rho$ represents the droplets density, $d_{s}$ the small droplets initial diameter, $d_{l}$ the large droplets initial diameter, $V_{r}$ the relative droplets velocity, $\sigma$ the surface tension and $\delta$ the dimensional impact parameter.

The dimensional impact parameter is defined as the distance from the centre of one droplet to the relative velocity vector placed on the centre of the other droplet as illustrated in figure 3.6.

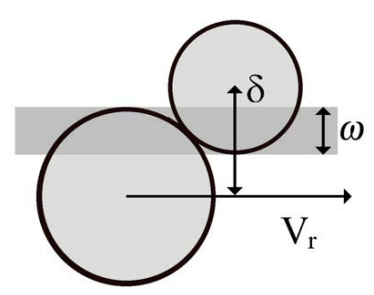

Figure 3.6: Definition of dimensional impact parameter $\delta$ and the width of the overlapping interaction region $\omega$.

Most papers describe the collision of equal sized droplets, with both droplets consisting of the same material. But in this specific case the colliding droplets consist of two different materials and both droplets are different in size. Estrade et al. [54] is an exception; the droplets still consist of the same material, the colliding droplets are of different sizes. An overview of the phase diagram for different impact regimes (figure 3.7) is given with a diameter ratio between both droplets of 0.5. The represented curves are derived heuristically, curve A predicts the bouncing effect [54], B the reflective separation effect at lower impact parameters [53] and C predicts the separation at high impact parameters $[53,55]$. Equations for these curves can be found in appendix $C$. 


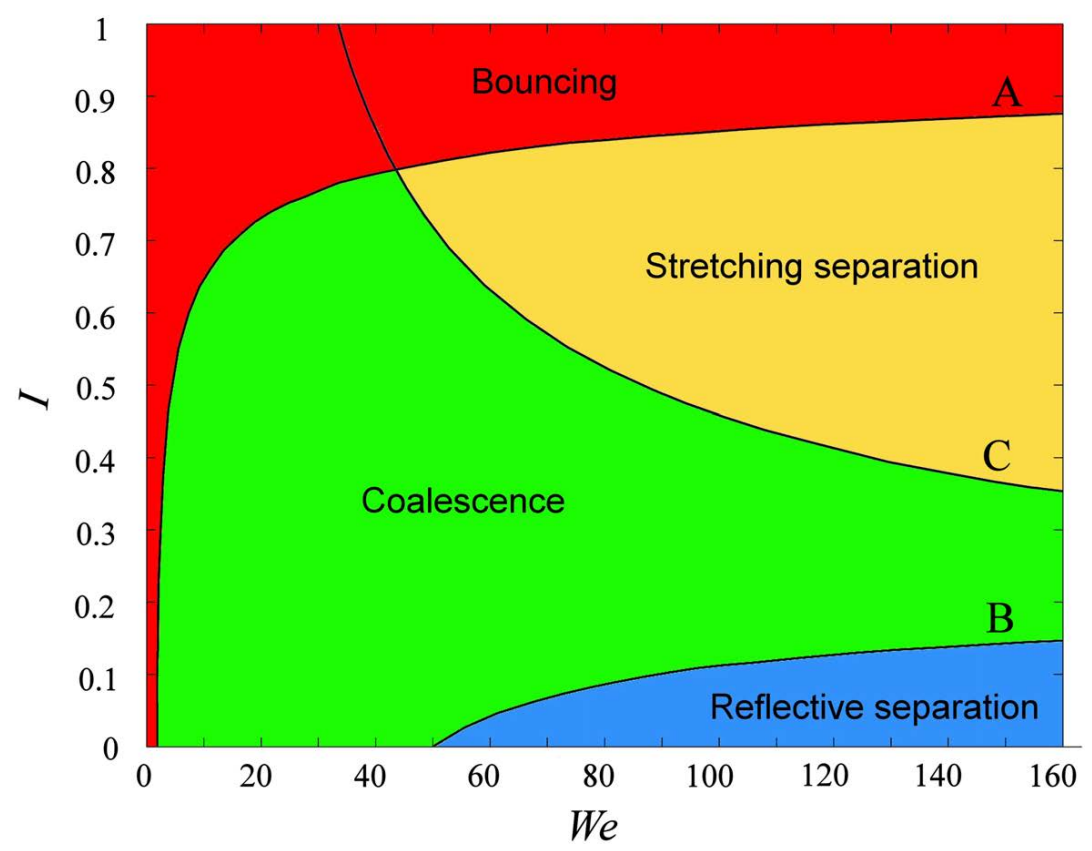

Figure 3.7: Regions of different collision outcomes based on experimental results at ambient pressure in air: bouncing (red), coalescence (green), stretching separation (yellow), reflective separation (blue) [54].

These prediction curves are based on droplets of equal material. If two different materials are used, the We number is not well defined with formula 3.1 since it is not clear which surface tension and density to use. Chen [56] investigated diesel-ethanol droplets collisions of equal size and defined the We number based on an average density $\rho$, where the average droplet density is derived from linear momentum conservation and defined as:

$$
\rho=\frac{2 \cdot \rho_{D} \cdot \rho_{E}}{\rho_{D}+\rho_{E}}
$$

where $\rho_{D}$ represents the density of diesel and $\rho_{E}$ the density of ethanol. For the surface tension the highest of both is selected $\left(\sigma_{D}=28.3 \cdot 10^{-3} \mathrm{~N} / \mathrm{m}\right.$, compared to $\sigma_{E}=22.1 \cdot 10^{-3} \mathrm{~N} / \mathrm{m}$ ). Chen argues that this does not always represent the correct physics underlying the collisions behaviour. The strength of the liquid bridge formed between both droplets after collision determines whether both droplets merge (coalescence) or detach again (separation). It is noticed that the material with the lowest 
surface tension spreads rapidly over the surface of the colliding droplet so one would expect the lower surface tension to be dominant. However, depending on the type of collision this liquid bridge behaves differently. It is discussed that especially at low We numbers the choice of relating the We number to the highest surface tension is not correct for predicting the boundary between reflective separation and coalescence. The liquid bridge is not uniform; one side of the bridge containing mostly diesel, the other side containing mostly ethanol. This results in a more fragile bridge, showing reflex separation at lower We numbers than predicted.

This in contrary to the effect noticed at the boundary between stretching separation and coalescence, appearing at higher impact parameters, where the formed bridge is significantly thicker due to the spreading of the low surface tension fluid. The larger thickness of the liquid bridge is more dominant to the strength of the bond between both droplets than the decreased surface tension, resulting in a larger regime of coalescence at the same $W e$ number. If the lowest surface tension would be chosen to determine the We number, only the reflex separation regime at low We numbers would be predicted more accurately, while the different results at higher We numbers would still remain. Therefore it is chosen to use We numbers equal to Estrade et al. and Chen to allow comparison of the results. The We number is defined as equation 3.1 based on the average droplet density as represented in equation 3.3 using the highest surface tension.

With droplet collisions in the bouncing (red in figure 3.7) and stretching separation (yellow in figure 3.7) regime, both fluid streams appear to remain separate. For these impact regimes the return flow does not seem to be contaminated and therefore preferred. However, Chen argues that even in the stretching separation regime the low surface tension fluid rapidly spreads towards the other droplet. Therefore, depending on the fluids used, even in the stretching separation regime one of both fluid flows becomes contaminated. If the surface tension of the drop-on-demand droplet can be chosen higher than the surface tension of the high viscosity droplets, the recycle of the continuous inkjet remains uncontaminated. If the deflection angles in the bouncing and stretching separation regimes appear to be too small and coalescence of droplets is necessary to achieve selection, the usage of immiscible fluids might still be an alternative enabling easy recycling of the return flow.

With respect to the bouncing regime, the effect relies on a small portion of the surrounding medium being captured between both droplets. As long as the relative velocity of both droplets doesn't overcome the raising pressure build-up bouncing takes place [57]. The occurrence therefore is dependent on the surrounding medium and the pressure thereof and it can be expected that increasing the surrounding pressure might broaden the range of bouncing collision outcomes. However, since increasing 
the ambient pressure in a printing application is not straight forward this possibility is not considered in more detail.

In the following paragraph an experimental setup is described to investigate whether droplet deflection using colliding droplets is possible and whether bouncing and stretching separation regimes can be obtained and sufficient deflection can be achieved.

\subsubsection{Experimental setup}

In figure 3.8 a picture of the experimental setup is shown. A continuous inkjet printhead is combined with a single nozzle DOD printhead. In this specific case a MicroFab MJ-AT DOD generator is used with a $50 \mu \mathrm{m}$ nozzle. The device is electronically controlled using MircoFab JetDrive III drive electronics. The CT-PT-SJ from MicroFab is used as a backpressure regulator. The continuous printhead uses a $50 \mu \mathrm{m}$ nozzle and the material feed as described in chapter 2 . The piezo element from the continuous printhead is driven by a TI signal generator using a PI505 piezo amplifier. To enable droplet selection the sync output from the signal generator is led into a pulse divider, to select one out of 64 droplets. The divider triggers a TI pulse generator, allowing a variable pulse delay towards the JetDrive III, enabling exact collision timing. Another TI pulse generator is used to trigger the cameras, an and-port is used to synchronize the illumination, again allowing a variable pulse delay, so that different moments of the collision can be visualized. A schematic representation of the connected electronics can be found in figure 3.9.

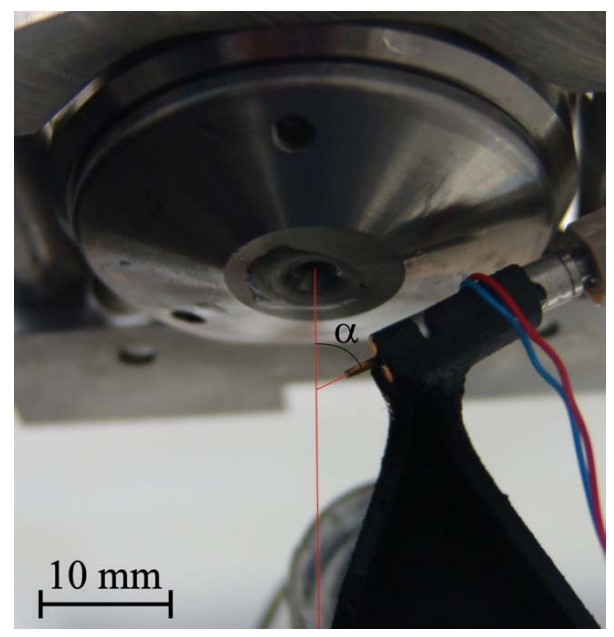

Figure 3.8: Experimental setup for droplet collision; the drop-on-demand printhead is placed under an angle $\alpha$ of $60^{\circ}$ with respect to the continuous jet. 


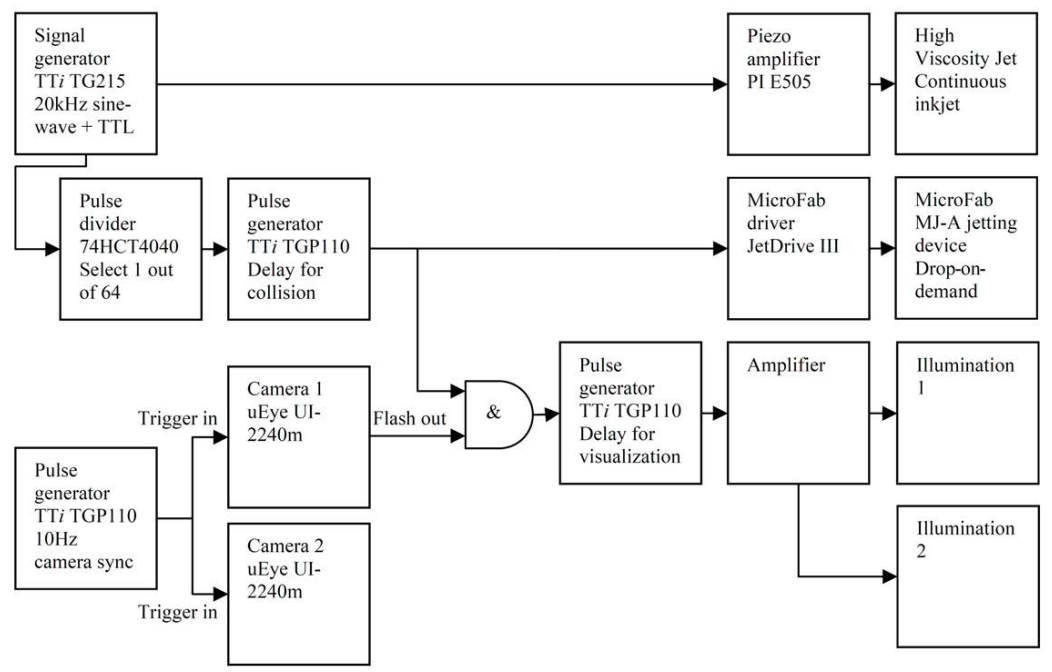

Figure 3.9: Schematic representation of the connected electronics.

\subsubsection{Experimental results}

During the experiments the high viscosity jet processed tri-ethylene glycol and was set to generate droplets of $110 \mu \mathrm{m}$ in diameter with a speed of $8.8 \mathrm{~m} / \mathrm{s}$ [58]. For the drop-on-demand printhead a material with a higher surface tension was chosen; demineralised water. This printhead was set to generate droplets with a diameter of $55 \mu \mathrm{m}$ and a speed of $1.5 \mathrm{~m} / \mathrm{s}$. To calculate the impact parameter two moments in time before the impact were selected. At each moment four pictures were taken. On the basis of these four images the mean droplets positions and dimensions were calculated. The difference in these mean positions was used to interpolate the expected droplets path to calculate the impact parameter. The droplet sizes were very stable during experiments, the continuous inkjet however showed a jitter up to $15 \mu \mathrm{m}$ resulting in an impact parameter accuracy of \pm 0.18 . In figure 3.10 two outcome regimes of the collisions are illustrated. The images show droplets at four moments in time, based on photographs of the droplets. In green the continuous and DOD droplet at $\mathrm{t}=0 \mu \mathrm{s}$, followed by blue at $\mathrm{t}=190 \mu \mathrm{s}$, red at $\mathrm{t}=510 \mu \mathrm{s}$ and black at $\mathrm{t}=690 \mu \mathrm{s}$. The left image shows the coalescence impact regime with an impact parameter of 0.14 where both droplets merge into one. In the right image bouncing occurs. The water droplet collides with the tri-ethylene glycol droplet with an impact parameter of 0.93 and bounces of without any fluid mixing. 


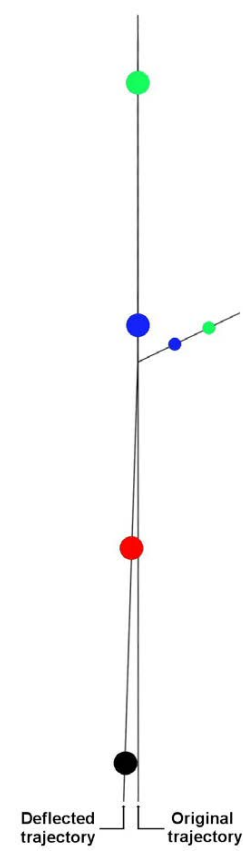

(a) Coalescence, $\quad I=$ 0.14 . The water droplet coalescences with a triethylene glycol droplet. The droplets continue their path jointly.

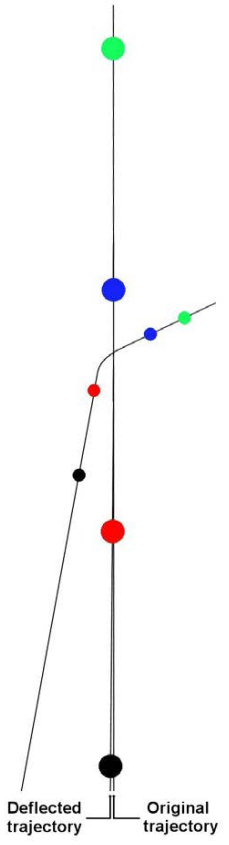

(b) Bouncing, $I=0.93$.

The water droplet bounces of the triethylene glycol droplet.

Figure 3.10: Illustration of coalescence and bouncing based on experimental data at $\mathrm{t}=0 \mu \mathrm{s}, \mathrm{t}=190 \mu \mathrm{s}, \mathrm{t}=510 \mu \mathrm{s}$ and $\mathrm{t}=690 \mu \mathrm{s}$.

For the experimental series fixed We numbers were chosen and the impact parameter was varied by changing the moment the drop-on-demand printhead was fired. Table 3.2 gives information about the fluid properties from the fluids used while table 3.3 gives an overview of initial droplet conditions and the resulting $W e$ numbers calculated using equation 3.1. The resulting collision outcomes are displayed in figure 3.11 together with the boundary lines defined by Estrade et al. The collisions resulting in a deflection angle larger than $2^{\circ}$ are marked yellow. 
Table 3.2: Fluid parameters.

\begin{tabular}{|l|c|c|}
\hline & Tri-ethylene glycol & Demi-water \\
\hline Chemical description & $\mathrm{C}_{6} \mathrm{H}_{12} \mathrm{O}_{4}$ & $\mathrm{H}_{2} \mathrm{O}$ \\
\hline Viscosity $[\mathrm{Pa} \cdot \mathrm{s}]$ & $33.8 \cdot 10^{-3}$ & $1 \cdot 10^{-3}$ \\
\hline Surface tension $[\mathrm{N} / \mathrm{m}]$ & $42.5 \cdot 10^{-3}$ & $72.8 \cdot 10^{-3}$ \\
\hline Density $\left[\mathrm{kg} / \mathrm{m}^{3}\right]$ & $1.124 \cdot 10^{3}$ & $0.998 \cdot 10^{3}$ \\
\hline
\end{tabular}

Table 3.3: Parameters of the initial droplets during the experiments.

\begin{tabular}{|c|c|c|c|c|c|c|}
\hline & \multicolumn{2}{|c|}{ Series 1} & \multicolumn{2}{|c|}{ Series 2} & \multicolumn{2}{|c|}{ Series 3} \\
\hline & CIJ & DOD & CIJ & DOD & CIJ & DOD \\
\hline Velocity [m/s] & 8.4 & 1.5 & 8.6 & 1.3 & 11.0 & 1.6 \\
\hline Droplet diameter [m] & $\begin{array}{l}110 \\
10^{-6}\end{array}$ & $\begin{array}{l}55 \\
10^{-6} \\
\end{array}$ & $\begin{array}{l}110 \\
10^{-6}\end{array}$ & $\begin{array}{l}55 \\
10^{-6}\end{array}$ & $\begin{array}{l}110 \\
10^{-6}\end{array}$ & $\begin{array}{l}55 \\
10^{-6} \\
\end{array}$ \\
\hline $\begin{array}{l}\text { Inter-droplet-distance } \\
\text { [m] }\end{array}$ & $\begin{array}{l}275 \\
10^{-6}\end{array}$ & & $\begin{array}{l}330 \\
10^{-6}\end{array}$ & & $\begin{array}{l}440 \\
10^{-6}\end{array}$ & \\
\hline Distance DOD-CIJ [m] & \multicolumn{2}{|c|}{$3 \cdot 10^{-3}$} & \multicolumn{2}{|c|}{$3 \cdot 10^{-3}$} & \multicolumn{2}{|c|}{$4 \cdot 10^{-3}$} \\
\hline We number & \multicolumn{2}{|c|}{48} & \multicolumn{2}{|c|}{51} & \multicolumn{2}{|c|}{85} \\
\hline
\end{tabular}

\subsubsection{Discussion}

Two aspects will be discussed; first the fit of the collision outcomes to the boundary curves from Estrade et al. with respect to Chen. Second the resulting deflection angles and the applicability of ballistic droplets selection.

From the experiments, it can be observed that, at high We numbers coalescence is preferred over stretching separation compared to the prediction lines of Estrade. This can be explained by the relatively large ligament which is formed due to the easy spreading of the low surface tension material compared to the surface of the other droplet, analogue to the results of Chen. At low We numbers, stretching separation is more preferred than coalescence. This difference compared to the results of Chen might be explained by the larger difference in surface tension between both colliding droplets (Chen: $\sigma_{h} / \sigma_{l}=1.28$ compared to the current experiments: $\sigma_{h} / \sigma_{l}=1.71$ ). At decreasing $W e$ numbers the formed ligaments are smaller, and the surface tension effects probably become dominant over the ligament size increase. A difference between the prediction curve and the experiments for the bouncing curve can be expected since the bouncing regime is not only dependent on $W e$ and $I$, but also for a large fraction on the vapour phase between both colliding droplets.

For droplet selection a deflection angle of $2^{\circ}$ was required, preferably in the bounc- 


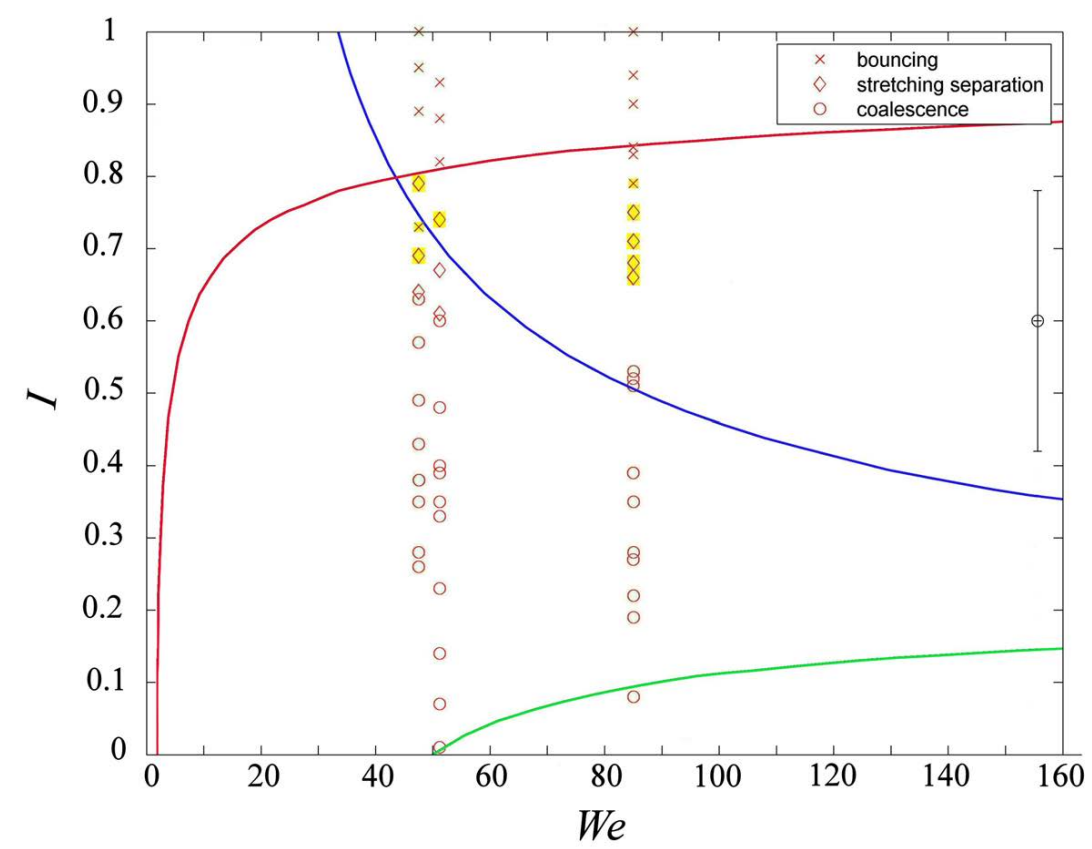

Figure 3.11: Experimental collision outcomes combined with the boundary curves from Estrade et al. [54] (marked yellow deflection angle $>2^{\circ}$ ).

ing regime where no material contamination would occur. From figure 3.11 it can be seen that only few experiments show large enough deflection angles, most in the stretching separation regime, resulting in contamination of the drop-on-demand material. Two bouncing outcomes showed a deflection angle large enough; one at $W e=48$ with $I=73$ and another one at $W e=85$ with $I=71$, however in both cases bouncing was very unstable, switching between bouncing and stretching separation, due to fluctuations in the jet speed of the printhead during these specific experiments, the impact parameter varied between both regimes.

It can be stated that deflection of droplets using ballistic deflection is achievable in the stretching separation regime, most stable at higher We numbers. However, the collisions barely reach the $2^{\circ}$ minimum deflection angles. To increase the deflection angle one could investigate collisions with higher We numbers using larger drop-ondemand droplets. Due to the complexity of the system and narrow operating window, the need for an alternative deflection mechanism remains. Therefore deflection using an air-jet will be investigated in the next paragraph. 


\subsection{Droplet selection by air-jet}

Blowing against a stream of droplets will also alter the droplet's path. Creating a droplet selection mechanism using an airflow seems straight forward. But creating a single droplet selection mechanism becomes challenging. It requires the airflow to be very small, since only one single droplet should be affected. Also the pulsation time must be very short whilst still achieving enough deflective energy; the airflow may only interact with one passing droplet. In WO 2009061195 [25] several methods for such a single droplet selection are described. Continuous starting and stopping of the airflow might be obtained at $20 \mathrm{kHz}$ repetition frequency, although it becomes problematic to start and stop the flow instantaneously on-demand. Therefore it is chosen to use a continuous airflow which is selectively placed in the path of the droplet stream (figure 3.12).

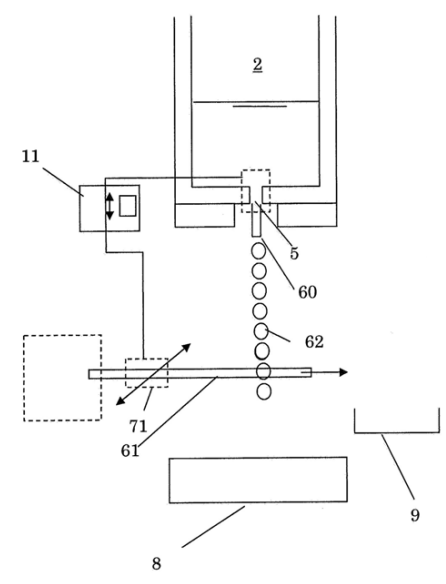

(a) A printhead (2) ejects a continuous stream of droplets from nozzle 5 . A timing circuit (11) triggers a mechanism (71) directing a continuous jet towards the created droplets from printhead 2. The collision with the jet causes the droplet to diverge from its initial path and is collected in gutter 9 . Non collided droplets reach substrate 8 undisturbed.
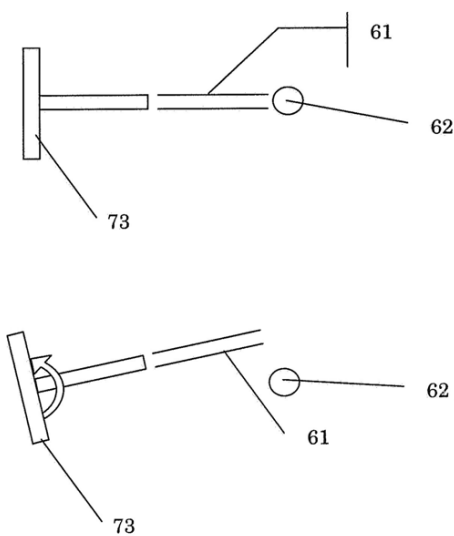

(b) An embodiment where the jet (61) is aimed at droplet stream 62. To allow rapid switching of the jet the origin of the jet (73) is rotated in the image right-below for the jet 61 to clear the droplet stream 62. The jet is not switched of, only rotated away; a small rotation angle already clears the path of the droplets.

Figure 3.12: Droplet selection mechanism [25]. 
To generate a working selection mechanism based on an air-jet, two elements need to be investigated. First, the air-jet itself; can an air-jet be created that only influences one single droplet at the time, and still result in a droplet deflection angle of $2^{\circ}$ ? Secondly, the air-jet movement mechanism; can a mechanism be created that can move the air-jet fast enough so that only one single droplet is deflected?

These questions are connected. From the point of view of transferring energy towards the droplet, a large air-jet is preferred. The larger the jet, the longer the passing droplet is in contact with the airflow, resulting in a larger deflection angle. However, if the air-jet is larger, there is less time to move the air-jet between the passing droplets. So from dynamics point of view a very small air-jet is preferred. To achieve insight in the sizes and timescales, the selection process of a single droplet will be studied in more detail. In figure 3.13 a schematic representation of different phases in the selection sequence is shown.

The time available for the movement $t_{m}$, is dependent on the distance $l_{d}$ between both droplets, the speed of the droplets $v_{d}$ and the diameter of the air-jet $d_{j}$ and can also be expressed based on the droplet generation frequency $f$ and droplet diameter $d_{d}$ according to:

$$
t_{m}=\frac{\left(l_{d}-d_{j}\right)}{v_{d}}=\frac{\left(\frac{v_{d}}{f}-d_{d}-d_{j}\right)}{v_{d}}
$$

The aim of the expression is to achieve insight in the dependency of the variables. They still remain approximations, due to the cylindrical shape of the air-jet and the spherical shape of the droplet (the time available is slightly longer).

Substituting the printhead settings as used in the previous paragraph in equation 3.4 ( $110 \mu \mathrm{m}$ droplets generated at $20 \mathrm{kHz}$ with a velocity of $10 \mathrm{~m} / \mathrm{s}$ ), the theoretical allowable air-jet dimensions in this specific example can be derived, ranging from $0 \mu \mathrm{m}$ to $390 \mu \mathrm{m}$ with an allowable movement time between $39 \mu \mathrm{s}$ and $0 \mu \mathrm{s}$ respectively.

To create as much time as possible for the movement of the air-jet, experiments have been performed to investigate the deflection angle of small air-jets. First stationary air-jets will be investigated with its effect on passing droplets. Thereafter the air-jets movement mechanism will be described in more detail. 


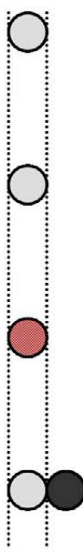

$t_{1}$
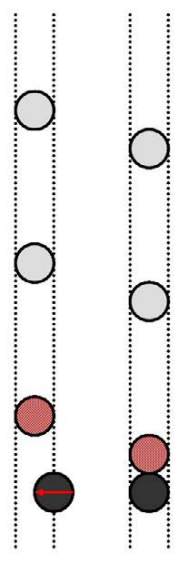

$\mathrm{t}_{2}$

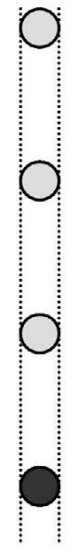

$\mathrm{t}_{5}$
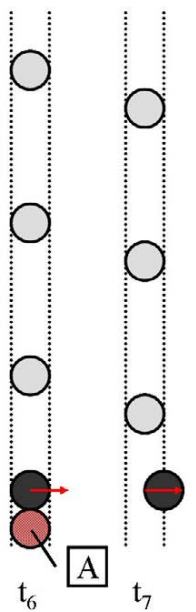

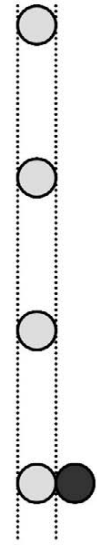

$\mathrm{t}_{9}$

Figure 3.13: A schematic representation of different phases in the selection sequence. The droplet stream passes from top to bottom (the droplets are displayed in light gray), from left to right several moments in time are represented. In this specific representation the red droplet is going to be deflected. At $t_{1}$ the air-jet (dark circle) is aimed to pass the droplet trajectory, just missing the passing droplet. At $t_{2}$ the previous droplet has passed and the movement of the air-jet can start. At $t_{4}$ the airjet needs to be at the desired position, contacting the droplet until $t_{6}$. The droplet marked $A$, has been affected by the air-jet and has moved. Thereafter the air-jet needs to clear the path of the following droplet, the sideways movement starts and needs to be finished at $t_{8}$ so that the next droplet can pass undisturbed at $t_{9}$.

\subsubsection{Performance of small stationary air-jets}

In the previous section an estimation was made about the allowable dimensions of the air-jet. When the size of the air-jet is fixed, two variables can still be selected that influence the deflection angle of the droplets; the material ejected from the jet, and the velocity at which this is ejected. Both are of influence to the force $F$ exerted on the droplets according to equation 3.5 [59].

$$
F=1 / 2 \cdot \rho \cdot v^{2} \cdot A \cdot C_{w}
$$

where $\rho$ represents the density of the surrounding medium, $v$ the speed of the air-jet, $A$ the projected surface of the droplet perpendicular to the flow direction and $C_{w}$ the drag coefficient. 
The material ejected: The usage of plain air would be the most convenient. In that case no extra gas supply would be required for the deflection system, only a small air pump. The specific density of air is relatively high compared to other gasses making it from an efficiency point of view a logical choice. Alternatively carbon dioxide could be used which has even a higher specific density $\left(1.97 \mathrm{~kg} / \mathrm{m}^{3}\right.$ compared to 1.29 $\mathrm{kg} / \mathrm{m}^{3}$ for air) but due to practicality plain air is preferred.

The velocity of the air: From equation 3.5 one would prefer an airspeed as high as possible. However one has to consider that it is necessary that the jet remains selective, meaning it should only effect one single droplet. High airspeeds might lead to turbulent behaviour reducing selectivity. Figure 3.14 shows two flow regimes made visible by ejecting smoke through the needle. During the first regime the diameter of the airflow remains virtually identical to the inside diameter of the supplying needle. In contrary to the second regime, where the flow turbulently mixes with the surrounding air, broadening the flow profile rapidly. Depending on the supplied pressure the length of the first regime can be varied; the higher the airspeed, the shorter the resulting first regime. It must be stated that only the movement of the supplied air is visualised, the effect of the air-jet on the surrounding air is not visible using this method. Therefore more elaborate visualisation methods are required such as schlieren and shadowgraph techniques [60].

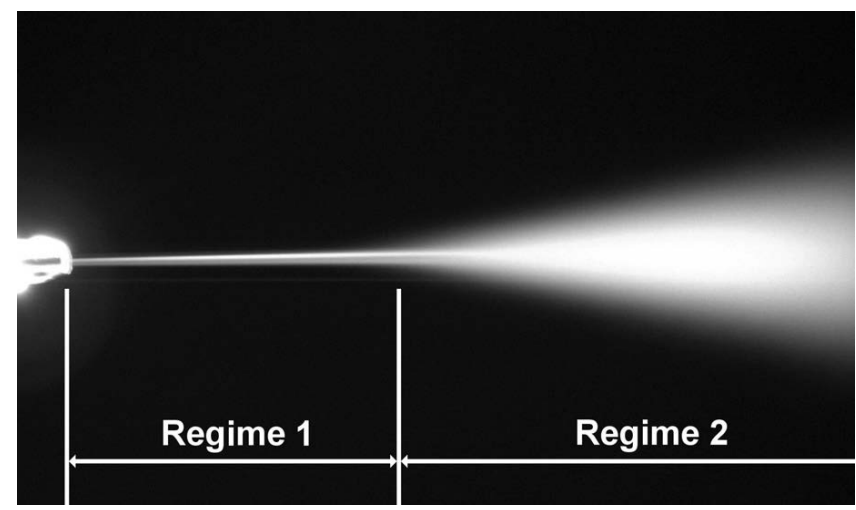

Figure 3.14: Smoke pressurized out of a 30 gauge needle (supply pressure 840 mbar).

Another effect which occurs when subjecting droplets to increasing airspeeds is the disintegration of the droplet. Several publications describe the deformations occurring, the regimes are described with increasing $W e$ numbers based on experimental results [61-66]. Where the We number is defined based on the density and speed 
of the gas, and the diameter and the surface tension of the droplet. The following regimes, illustrated in figure 3.15 , occur:

1 Vibrational

2 Bag breakup

3 Multimode breakup

4 Sheet thinning

5 Catastrophic breakup or shattering

$$
\begin{aligned}
0<W e< & \sim 11 \\
\sim 11<W e< & \sim 35 \\
\sim 35<W e< & \sim 80 \\
\sim 80<W e< & \sim 350 \\
W e> & \sim 350
\end{aligned}
$$

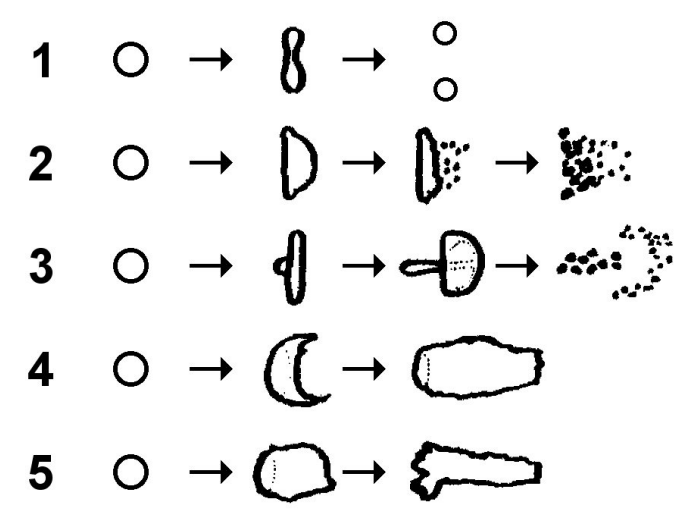

Figure 3.15: Droplet deformation and breakup occurring with increased We numbers [62].

It is stated that viscosity effects are not significant if the $O h$ number is less than 0.1 $[63,67]$.

$$
O h=\frac{\mu_{d}}{\sqrt{\left(\rho_{d} \cdot d_{d} \cdot \sigma\right)}}
$$

Here $\mu_{d}$ represents the fluid viscosity, $\rho_{d}$ the fluid density, $d_{d}$ the initial droplet diameter and $\sigma$ the surface tension.

For a water droplet of $110 \mu \mathrm{m}$ the viscosity effects can be neglected $(O h=0.0124)$. For a tri-ethylene glycol droplet with the same dimensions $O h=0.466$ and viscosity will play a roll in the resulting effects. Although it can be expected that the higher viscosity has a dampening effect on the deformations of the droplet, possibly delaying the destructive deformations [68]. To achieve clean deflection the vibrational regime 
is preferred since this is the only regime where the droplets remain intact, although disintegration of the droplet might be allowed if the resulting droplet spray can be cleanly recollected for recycling.

Experiments have been performed to investigate the achievable deflection angle and resulting droplet deformations using different airspeeds. Two commercial available needles sizes were used; the smallest available sizes have an internal dimension of $100 \mu \mathrm{m}$ (32 gauge) and $150 \mu \mathrm{m}$ (30 gauge). Figures 3.16 and 3.17 show the deflection angle dependent on supplied air pressure and the distance between the centre of the droplet trajectory and the centre of the air-jet. Four air pressures are displayed in each figure, the highest air pressure causes break-up of the droplets (bag break-up) but the generated satellite droplets are stable and the occurring situation can be used for droplets recycling. The second highest pressure is the boundary of vibrational regime, still maintaining individual droplets. The deflection angles achievable are significant and raise beyond the required $2^{\circ}$. When (stable) satellite formation is allowed deflection angles of up to $16^{\circ}$ are possible using a 30 gauge needle (figure 3.18). It can even be expected that satellite formation will be postponed up to higher jet velocities and consequently higher deflection angles, if higher viscous droplets are used.

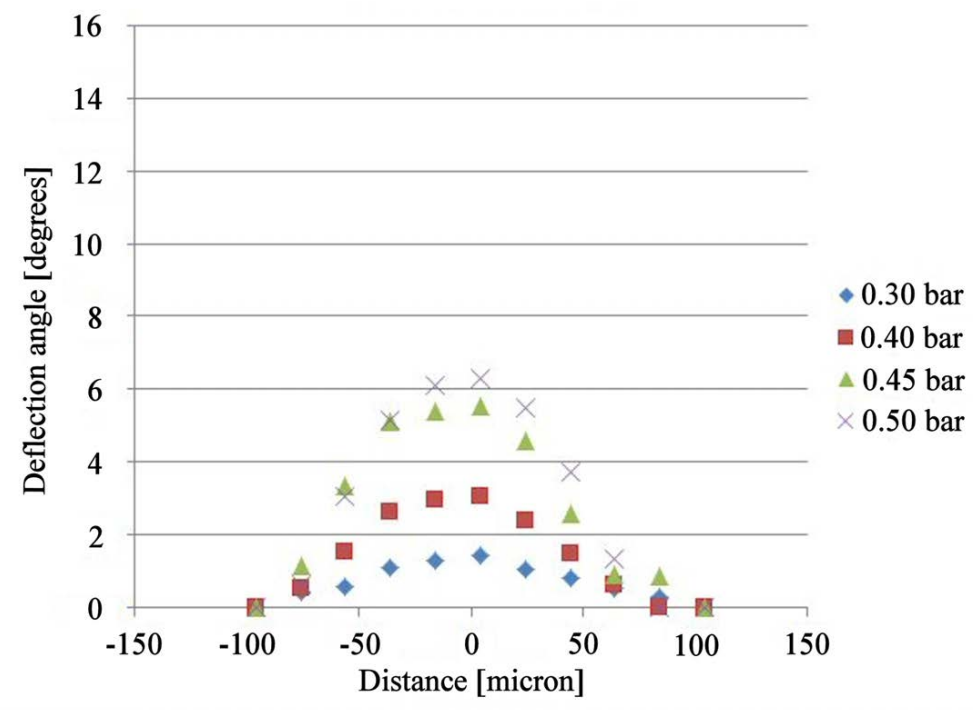

Figure 3.16: Deflection angles of 32 gauge needle deflecting $110 \mu \mathrm{m}$ IPA droplets travelling with $10 \mathrm{~m} / \mathrm{s}$ with different supply pressures. 


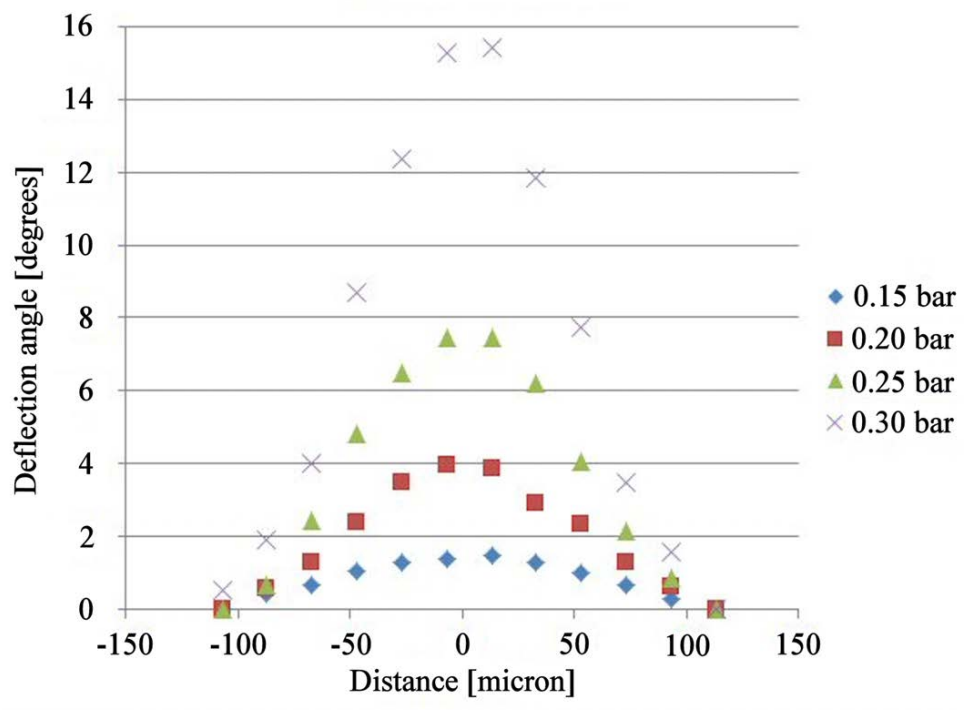

Figure 3.17: Deflection angles of 30 gauge needle deflecting $110 \mu \mathrm{m}$ IPA droplets travelling with $10 \mathrm{~m} / \mathrm{s}$ with different supply pressures.

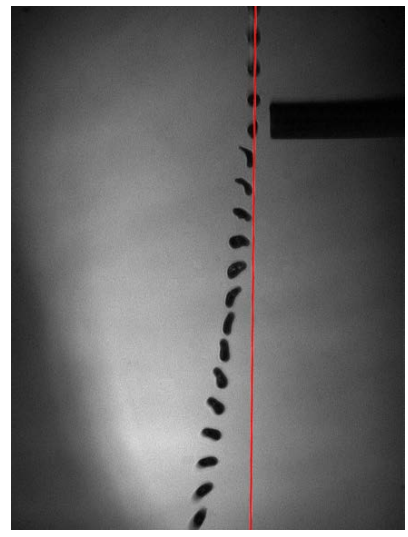

(a) 0.25 bar, vibrational regime, $7^{\circ}$ deflection.

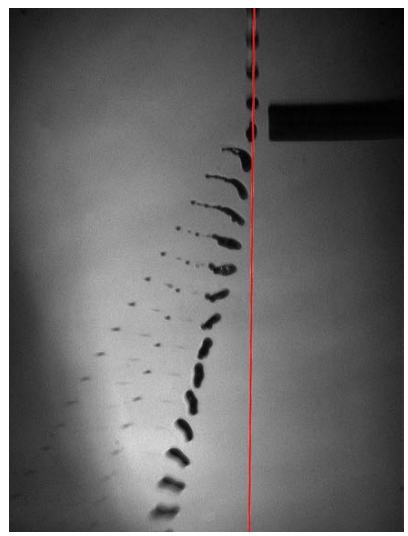

(b) 0.30 bar, bag break-up, $16^{\circ}$ deflection.

Figure 3.18: Droplet deflection using a 30 gauge nozzle, the red line represents the undisturbed trajectory. 


\subsubsection{Moving the air-jet}

Depending on the nozzle size used, $29 \mu \mathrm{s}$ (100 $\mu \mathrm{m}$ air-jet) or $24 \mu \mathrm{s}(150 \mu \mathrm{m}$ air-jet $)$ is available for the movement of the air-jet into the path of the droplet. The time available for deflection is $21 \mu \mathrm{s}$ (100 $\mu \mathrm{m}$ air-jet) and $26 \mu \mathrm{s}(150 \mu \mathrm{m}$ air-jet). If this would be a repetitive action one would design the system to be tuned to this specific repetitive frequency. However, the selection of the droplets is dependent on the printed image, resulting in the requirement of a movement which can be performed on-demand, independent on whether the previous droplet was deflected, or not. Figure 3.19 shows a piezo air nozzle actuator which has been realized with a (measured) eigen frequency of $16.7 \mathrm{kHz}$. No commercial available piezo amplifiers were found that were able to supply the required currents at the required operation frequencies. Therefore a binary piezo amplifier was developed capable of delivering the required currents. In theory, a step response should be achievable of $1 / 3$ of the lowest mechanical oscillation frequency by using binary input shaping [69], resulting in a minimum movement period of $20 \mu \mathrm{s}(60 \mu \mathrm{s} / 3)$.

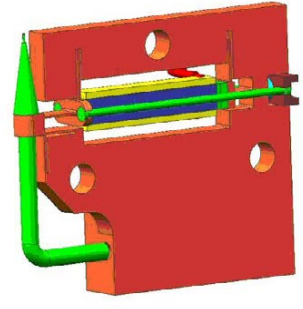

(a) Schematic representation of piezo actuated air nozzle.

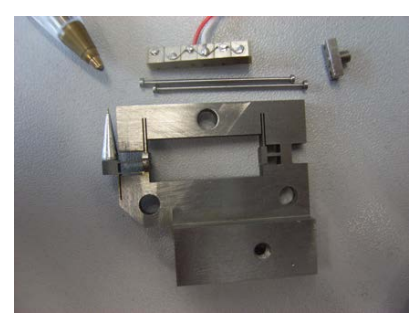

(b) Realized finished part.

Figure 3.19: Air nozzle actuation mechanism.

Input shaping is a feed forward control technique for reducing undesired oscillations in actuated mechanisms. The method works by means of creating a command signal that cancels its own vibration. The resulting vibration caused by the first part of the actuation signal is cancelled by the vibration caused by the second part of the actuation. If the impulses are chosen correctly, the system will respond without undesired vibrations. To validate whether the required response is achievable in practice, a test setup has been build to enable accurate measurements of the movement of the air-jet (figure 3.20). A laser twin sensor from LMI Technologies type LTS15/1 (high speed laser triangulation device) is used to measure the movement of the air nozzle. This specific sensor has a bandwidth of $200 \mathrm{kHz}$, with a measuring resolution down to $2.5 \cdot 10^{-8} \mathrm{~m}$. 


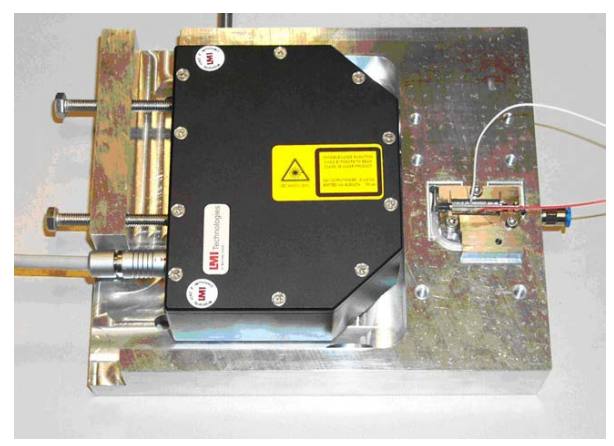

Figure 3.20: Piezo air-jet mounted in measuring frame together with the laser twin sensor.

Experimentally it was possible to shape the input waveform to enable a set up time of $20 \mu \mathrm{s}$ and maintain the position for $30 \mu \mathrm{s}$ before returning to the origin in the next $20 \mu \mathrm{s}$. In figure 3.21 the drive signal is shown (top graph) where clearly the input shaping is visible. The bottom graph represents the readout of the sensor showing the systems response. The achievable deflection is $70 \mu \mathrm{m}(700 \mathrm{mV} / 10 \mathrm{mV} / \mu \mathrm{m})$ at the measurement point, resulting in a maximum deflection of $85 \mu \mathrm{m}$ at the nozzle tip. The achievable $20 \mu$ s setup time would even allow a larger air-jet of $190 \mu \mathrm{m}$ to be used, however a $150 \mu \mathrm{m}$ is less critical and therefore preferred.

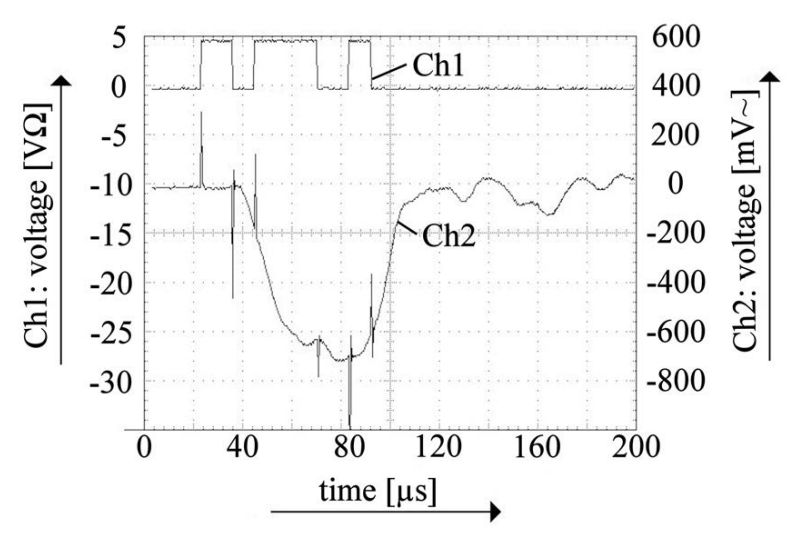

Figure 3.21: Input signal and resulting sensor readout. 


\subsubsection{Combination of the air-jet deflection mechanism with HVJ}

The air-jet actuation mechanism and air-jet are combined with the printhead to test the interaction of both systems. Figure 3.22a shows the deflection mechanism mounted beneath the printhead; it can be positioned by means of micro positioning stages to allow exact alignment with the droplet path. On the right the recycle gutter is shown, also movable to allow precise tuning to the droplets path. The figure shows a cooling duct being mounted underneath the actuator mechanism. This was necessary; otherwise the actuator would overheat within 10 seconds. The used electronics and signal processing is similar to the system described in figure 3.9, instead of timing the fire pulse of the MicroFab printhead the signal is used to trigger an arbitrary waveform generator supplying the signal as represented in figure 3.21 to drive the piezo element of the air-jet deflection mechanism.

Figure 3.22 gives a representation of successful droplet selection. The left figure shows the undisturbed droplets passing the recycle gutter, the right figure shows all but one droplet deflected into the recycle gutter. The droplets were generated using a $50 \mu \mathrm{m}$ nozzle operating at $20 \mathrm{kHz}$ generating $147 \mu \mathrm{m}$ droplets.

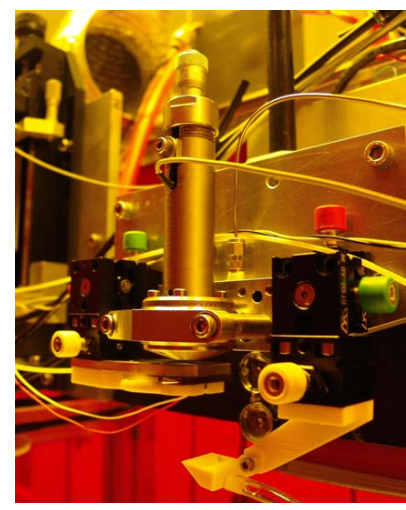

(a) Experimental setup to test air-jet droplet deflection.

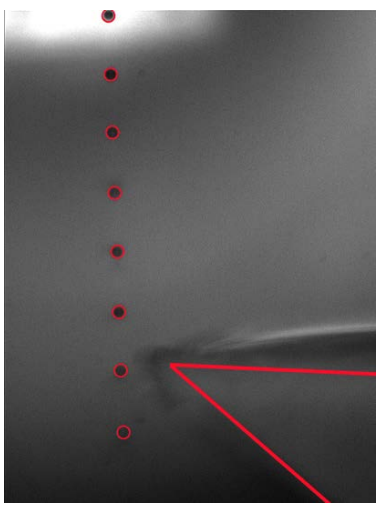

(b) Undeflected droplets and the recycle gutter.

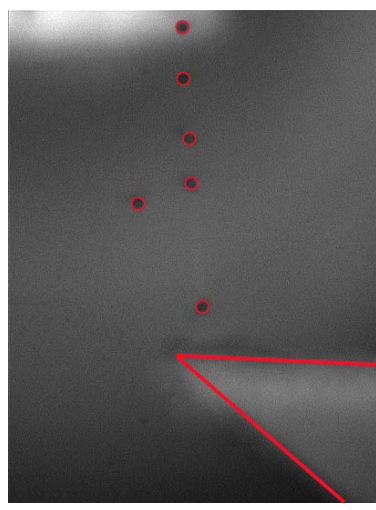

(c) All but one droplet being deflected into the gutter.

Figure 3.22: The air-jet deflection system and the deflection of $147 \mu \mathrm{m}$ droplets generated using a $50 \mu \mathrm{m}$ nozzle operating at $20 \mathrm{kHz}$. 


\subsubsection{Discussion}

An air-jet based droplet selection mechanism has been developed. The air-jets in the same dimensions as the generated droplets have sufficient influence on the droplets path to achieve the minimum $2^{\circ}$ deflection angle. The air-jet movement mechanism is capable of moving fast enough to enable on-demand single droplet selection. Input shaping and usage of a high current digital amplifier are necessary to achieve the required setup time. Combination of the air-jet actuator with the printhead results in a robust selection mechanism. The high deflection angles achievable, using the airjet deflection, gives room for decreasing the distance between nozzle and substrate, increasing overall system accuracy.

\subsection{Concluding remarks}

Comparing the air-jet selection principle to the ballistic droplet deflection; the air-jet droplet deflection system results in a more user friendly and stable situation. Using two printheads requires two droplets to collide at exactly the right position, while the air-jet only needs to contact the passing droplet, the only timing necessary is to prevent other droplets to be contacted as well. Also maintaining a second drop-ondemand printhead is relatively difficult, compared to the ease of creating a small air jet.

Still if possible electrostatic deflection is preferred due to its lack of moving parts and ability to function up to extremely high frequencies. If however due to limited conductivity electrostatic deflection is impossible, air-jet deflection creates a possibility to selectively deposit non conductive materials with droplet generation frequencies up to $20 \mathrm{kHz}$. 


\section{4 \\ Other applications of high viscosity jetting; spray drying \& droplet encapsulation $^{1}$}

The printhead, initially designed for the creation of $3 D$ products, can be used in other application areas as well. Generation of monodisperse droplets using the technology described in this thesis proves to be interesting for improving the spray drying process needed for the manufacturing of powders. The high viscosity materials which can be processed together with the control over the generated droplets (size, speed and direction) offers opportunities with respect to an increase in energy efficiency as

${ }^{1}$ The patents on which this chapter is based are filed as:

$\diamond$ A.T. Poortinga, R.J. Houben, "Method and apparatus for spray drying and powder produced using said method", (2008) WO2008069639. In cooperation with Royal FrieslandCampina.

$\diamond$ R.J. Houben, A. Rijfers, L.A.M. Brouwers, J. Eversdijk, K.J.C. van Bommel, "Multi component particle generation system", (2009), WO2009014432.

$\diamond$ F. de Vreede, A.P. Aulbers, R.J. Houben, "Pressure independent droplet generation", (2009), WO2009151332.

$\diamond$ R.J. Houben, A. Rijfers, L.A.M. Brouwers, "Multi component particle generating system", (2010), WO2010005302.

$\diamond$ R.J. Houben, A. Rijfers, L.A.M. Brouwers, "Multi component particle generating system", (2010), WO2010090518. 
well as more control over powder properties. Secondly, an encapsulation method is described where the droplets are jetted through a liquid screen creating encapsulates. Several examples are described to show the capabilities of the process.

\subsection{From droplet generation to monodisperse powders}

Several different powder production systems exist; most of them are based on the production of powder from a liquid, requiring an efficient drying mechanism. One commonly used powder production system is a so called spray dryer. A schematic representation is visualised in figure 4.1. An atomizer on top of the dryer generates a spray of small droplets. By maintaining an airflow past these droplets inside the drying chamber, the solvent evaporates from the droplets and powder particles are formed. The powder is subsequently removed from the airflow by means of a cyclone.

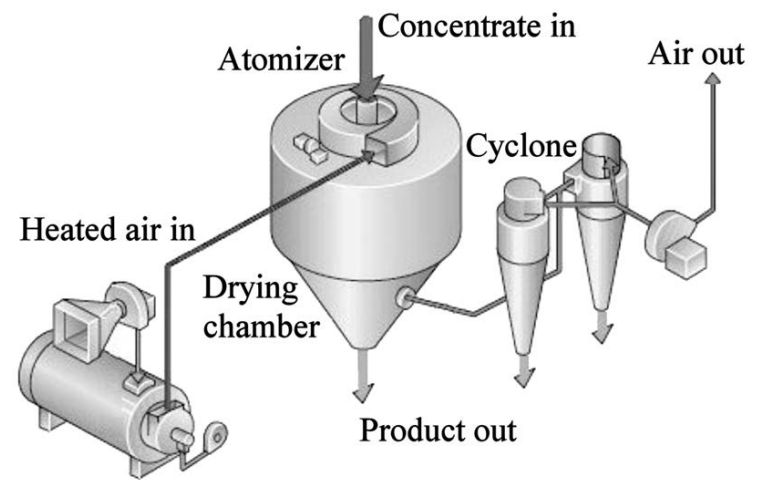

Figure 4.1: Schematic representation of a spray dryer.

The commonly used atomizers create a rather broad and uncontrolled size distribution of droplets. Large and small droplets are created simultaneously; the drying tower is designed to be able to dry the largest particle, resulting in overheating of the smaller particles. Very small droplets are formed as well, resulting in so called "fines", which are very difficult to separate from the airflow, and cause hazardous situations due to the increased risk of dust explosions. A more controlled way of creating the initial droplets is desirable. Therefore in WO2008069639: "Method and apparatus for spray drying and powder produced using said method" [26], the usage of high viscous inkjet technology for spray-drying is described. Figure 4.2 shows the schematic layout where the conventional atomizer is replaced by a printhead.

A typical industrial spray dryer has a drying capacity in the order of tons of solvent evaporation per hour. A single nozzle printhead has a material throughput of 


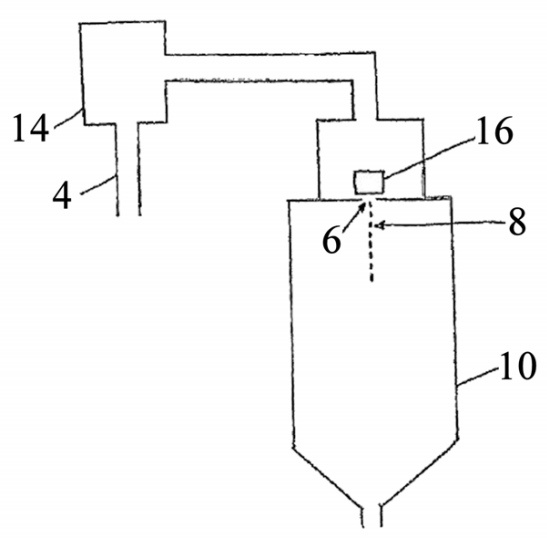

Figure 4.2: Method and apparatus for spray drying and powder produced using said method [26]. Cold air enters the system through tube 4, and is heated and preprocessed in section 14. The heated air is directed towards the spray tower (10) where a printhead (16) is located ejecting a stream of droplets (8) from nozzle(s) 6. The dried droplets leave the spray tower at the bottom of vessel 10 .

$0.15 \mathrm{~kg} / \mathrm{hour}$. Therefore scale-up is necessary to achieve a commercially applicable system. In collaboration with Royal FrieslandCampina the system is scaled-up towards a multi-nozzle system, capable of processing material feeds up to $100 \mathrm{~kg} / \mathrm{hour}$ to make the system applicable to pilot scale spray-drying facilities. The printhead design is based on the pressure independent setup as described in chapter 2.6.3. Figure 4.3 shows a schematic representation where next to the pressure independent design also the nozzle orientation is shown; where the nozzles are positioned under an angle with respect to the nozzle plate itself, resulting in a conical spray pattern. Figure 4.4 shows the multi nozzle printhead in action inside a drying tower, where also the conical spray pattern can be observed.

Several experiments were performed with the single nozzle [70] and multi nozzle printhead [71] at Royal FrieslandCampina to investigate the performance of the system. The results can be categorized by on one hand the effects with respect to the systems energy performance and on the other hand the effects of the process on the powder properties. 


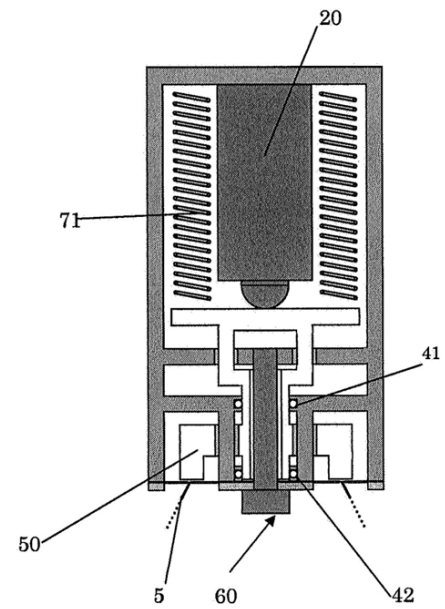

Figure 4.3: Schematic representation of the multi nozzle printhead [22] as described in chapter 2.6.3 using a pressure independent layout. The piezo element (20) is held under pretension by means of springs (71). The piezo presses against vibrating member 50 designed so the cross-sections of seals 41 and 42 result in a pressure neutral mounting of element 50. The assembly is mounted by a central mounting bolt (60) and the fluid is ejected out of the nozzle plate as jets (5). The jets leave the nozzle surface under an angle since the nozzle holes are laser-drilled especially under this angle to achieve a conical spray pattern. A further in-depth description of all noted numbers can be found in the patent application.

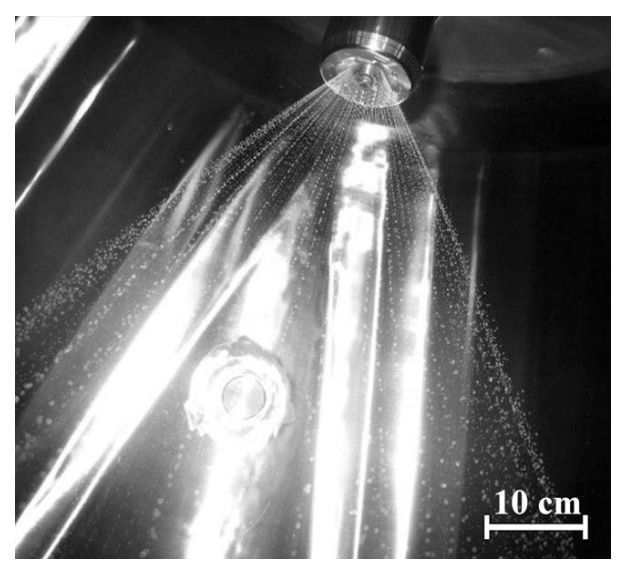

Figure 4.4: Multi nozzle printhead. 


\subsubsection{Energy efficiency}

High dry matter concentration: Conventional atomizers used in spray drying applications are, similar to conventional inkjet printers, limited in the viscosity they are able to process. Compared to an inkjet printhead an atomizer is capable of processing much higher viscosities; however it should be mentioned that the developed printhead is capable of processing even higher viscosities. Therefore the product can be fed towards the printhead with a higher dry matter concentration; consequently less water needs to be evaporated during the spray drying phase. For example for a creamer model formulation, the dry matter concentration can be increased from $62 \mathrm{wt} \%$ to 67 $\mathrm{wt} \%$. In another example, a high protein model formulation was used consisting of mainly whey, protein and lactose. Here an increase in solid content from $25 \mathrm{wt} \%$ to $35 \mathrm{wt} \%$ appeared to be possible. For product streams containing a large amount of water, the water still has to be removed from the feed before spraying. The increase in processable solid content seems redundant. But these feeds aren't directly fed to the spray dryers, pre-concentration systems are installed having a much higher energy efficiency, compared to the spray drying step itself. Being able to remove more water during pre-concentration results in a more energy efficient situation.

Monodispersity: Energy saving can also be achieved due to the fact that more monodisperse droplets are generated (figure 4.5). The drying step can then be optimized towards the specific droplet size. This is contrary to the current situation where the dryer is designed to be able to dry the largest droplets; wasting energy for most of the material.

Absence of fines: Although the absence of fines can not directly be seen as an energy reduction, it results in a more efficient usage of materials. With conventional systems, several percent of material is lost as fines and for the removal of these fines out of the airflow specific measures must be taken, such as the use of cyclones or complex filter systems. The absence of fines therefore results in a higher yield and less servicing of the filtration system.

\subsubsection{Powder properties}

Monodispersity: Monodispersity already has been proven to be an advantage with respect to energy efficiency. It can also be seen as an advantage for the controllability of the treatment of the powder particles. When the amount of drying energy is more in line with the particle size, there is less overheated material. Figure 4.5 shows the increase in monodispersity. The width of the particle size distribution was mea- 
sured by the span, defined as ([particle diameter at $90 \%$ cumulative size] - [particle diameter at $10 \%$ cumulative size]) / [particle diameter at $50 \%$ cumulative size]. A small span indicates a narrow size distribution. The relative span of the particle size distribution of 1-2 for a conventional powder can be reduced to $0.6-0.7$ for an inkjet produced powder.

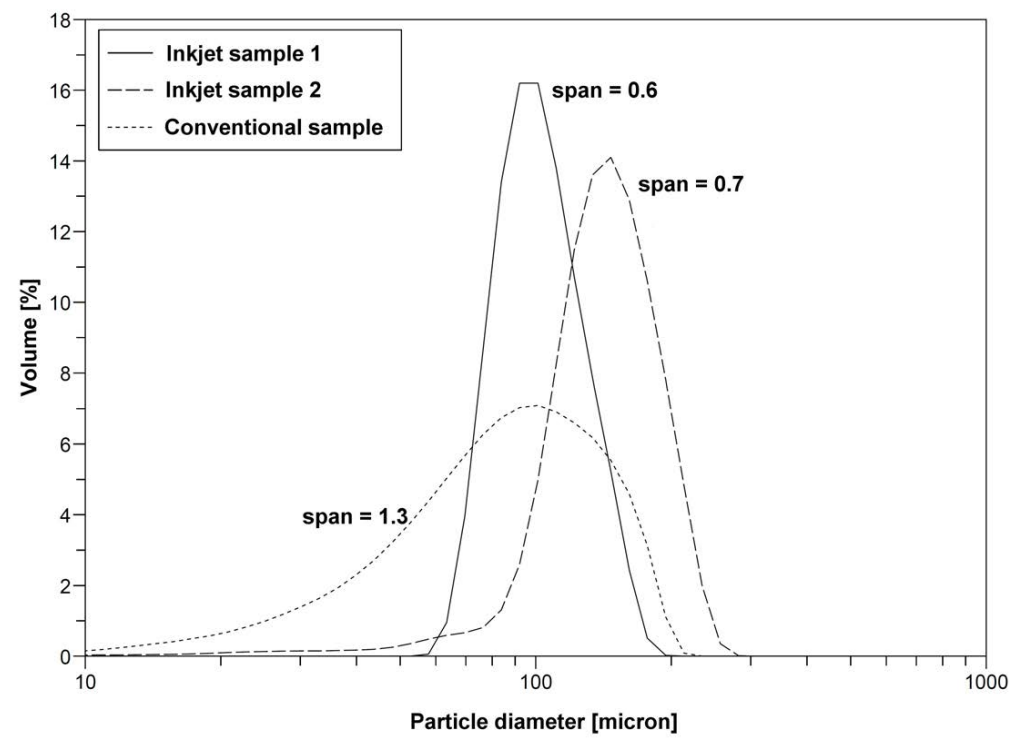

Figure 4.5: The reduced span of two different inkjet printed samples compared to the span of a conventional generated powder. Figure taken from [70].

Structure: When using conventional spray nozzles, very small particles are formed in a turbulent manner causing agglomeration during the drying stage, resulting in powder particles build up out of a several combined droplets, resulting in a porous powder structure. Using inkjet printing, the droplets are generated in a very controlled manner, avoiding droplet collisions, so the initial droplet becomes a single powder particle. The droplets generated by the inkjet contain no air-entrapments, resulting in solid particles. Also the generated powder consists of spherical particles (figure 4.6c and 4.6d), in contrary to the variety in shapes and morphologies that can be seen with conventional powders (figure 4.6a and 4.6b). This round particle shape results in an excellent flowability of the dried material. One has to keep in mind that although the absence of air entrapment is an advantage for the powder properties, it is much more difficult to dry a fully dense particle compared to a particle containing 
an air entrapment. Therefore longer drying times are required for massive droplets. This seems contra-dictionary to the claimed increase in energy efficiency, however, a longer drying time does not mean that more energy is used, it only takes more time to consume the energy delivered.

Density: The technology also creates more dense powders. For a model material consisting of maltodextrin a $50 \%$ density increase is measured, for a creamer based model material a density increase of $30 \%$ is realized.

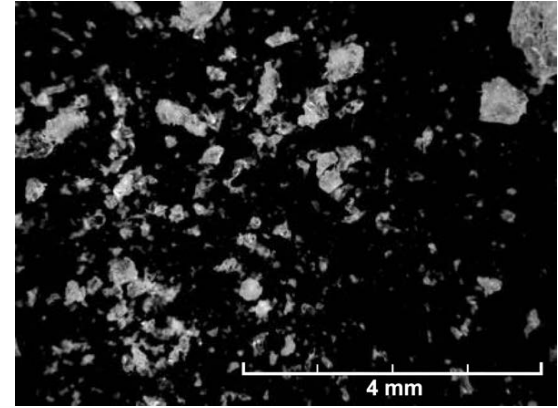

(a) Powder generated by means of a swirlflow nozzle

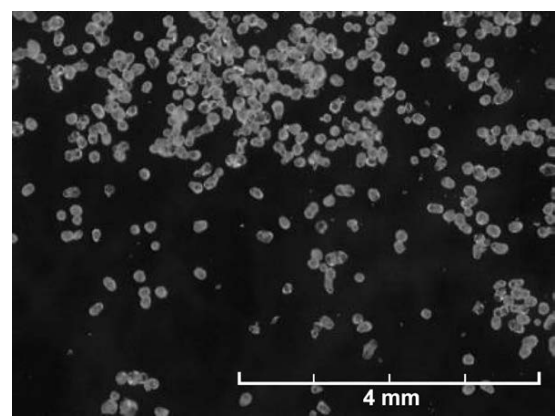

(c) Powder generated by means of the printing process

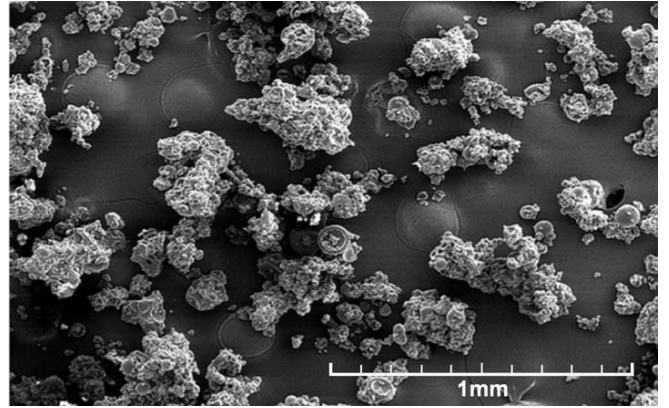

(b) Sem image of powder generated by means of a swirlflow nozzle

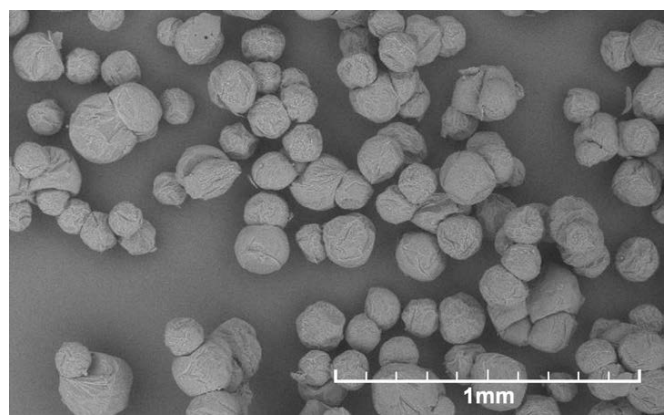

(d) Sem image of powder generated by means of the printing process

Figure 4.6: Differences between conventional powders and powders generated using inkjet printing

Mild processing: Processing sensitive emulsions through spray driers frequently proves to be impossible. The conventional high pressure or swirl nozzle cause the emulsion to be altered with respect to particle size. The printhead handles the mate- 
rials quite gently, resulting in the ability to process these sensitive emulsions while maintaining their desired emulsion size. Figure 4.7 shows a comparison of emulsion droplet sizes before and after inkjet and conventional processing.

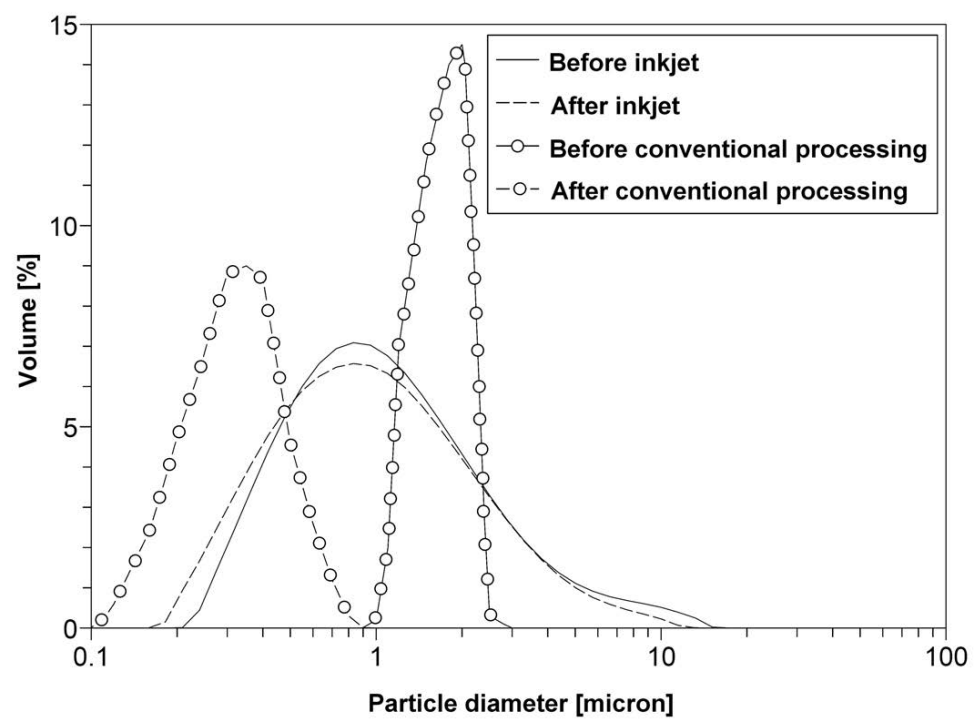

Figure 4.7: Emulsion droplet size distribution before and after processing [70].

\subsubsection{Discussion}

The results show the potential to use inkjet technology in spray drying applications. Two key issues remain:

Maintaining monodispersity: With the inkjet printing process it is possible to generate identical droplets, and it is desired to keep control over the droplets path as well, preventing droplet collision, resulting in monodisperse powders. In conventional spray drying a lot of small droplets are formed, and due to the random nature of the process a lot of collisions between droplets occur, leading eventually towards the mean particle size required (with a relatively large span around it). So conventional spray towers are not designed to maintain the initially generated droplets, droplet circulation of the particles is preferred to increase the contact between the air and the particle. When the collision between droplets needs to be prevented to maintain monodispersity a whole new view on tower design is required. 
Drying the generated droplets: The inkjet based process is a significantly different process compared to the conventional atomizers. The initial droplet formed should become the final powder particle, in contrary to the conventionally formed agglomerated particle consisting out of a large number of smaller initial droplets. Since it is more difficult to dry large droplets than small droplets, the usage of inkjet technology (although in principle it results in a much higher production efficiency) requires a taller drying tower to achieve dry powder.

To really exploit the benefits of inkjet produced powders the design of the spray drying tower needs to be reconsidered. Therefore the construction of a $15 \mathrm{~m}$ heigh drying tower has started within TNO for the build-up of a test facility specific for experiments using inkjet for the fabrication of powders (figure 4.8).

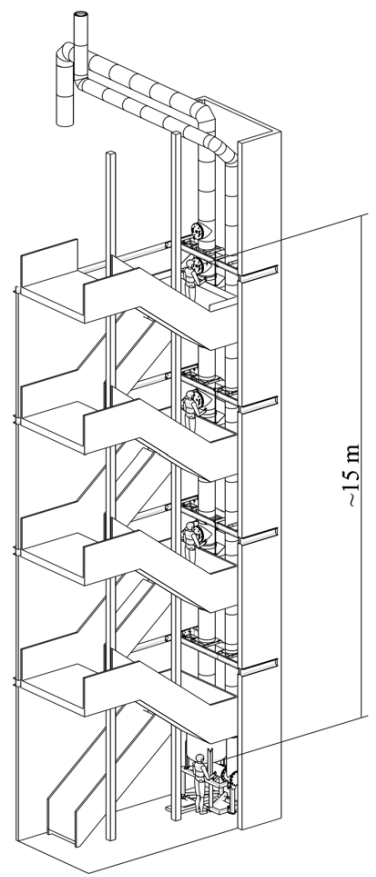

Figure 4.8: Spray tower under construction at TNO Eindhoven. 


\subsection{Inkjet as a means for the making of encapsulates}

In the previous paragraph inkjet has shown to be a promising technology for the production of powders. Its capability to constantly generate equal sized droplets is a unique feature for improved process control. Another feature not used for spray drying is the fact that not only the droplet size is known, but also the direction, timing, and velocity of the droplets. It can therefore also be used to develop a process for creating encapsulates. In WO2009014432 [27] a process is described using the high viscosity inkjet to produce encapsulates. The formed droplets are propelled through a liquid screen. During collision some of the screen material is transferred to the droplet. If the speed of the droplet is high enough, the droplet will break lose of the screen, exiting the screen on the opposite side as an encapsulate (figure 4.9).

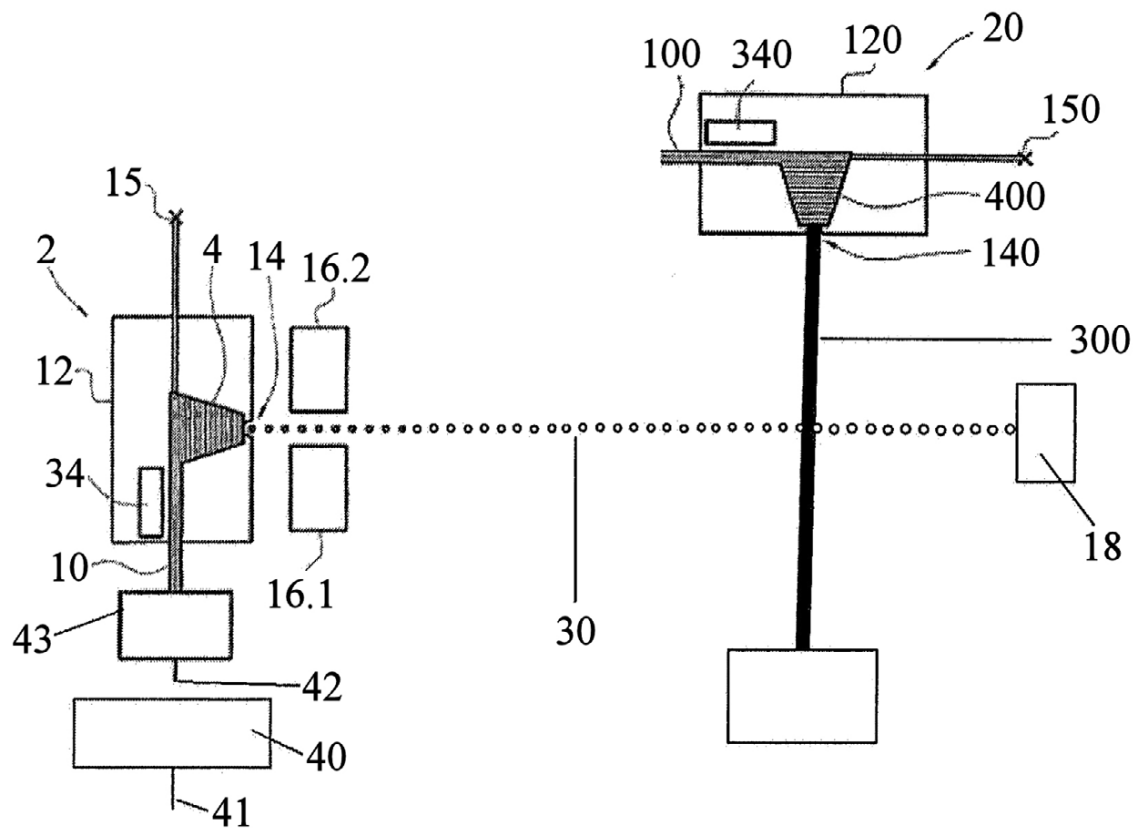

Figure 4.9: Multi component particle generating system [27]. A printhead (2) generates a continuous stream of droplets (30). Another ejection mechanism (20) generates a continuous fluid screen (300). Both are arranged in order for the droplets (30) to collide with the screen (300). The droplets break through the screen taking some of the screen material with them creating encapsulates. These encapsulates are collected in gutter 18. A further in-depth description of all noted numbers can be found in the patent application. 
Using this method it is possible to generate encapsulates, but one can also envision the two material streams to interact, for instance to cause demixing and form emulsions or cause precipitation, gelation or to start chemical reactions (figure 4.10). Due to the fact that both materials are brought into contact in discrete quantities during predefined timing intervals, the reactions proceed in a controlled manner. Even extreme exothermic reactions might be controlled due to the small reaction volumes, similar to micro-reactor technology.

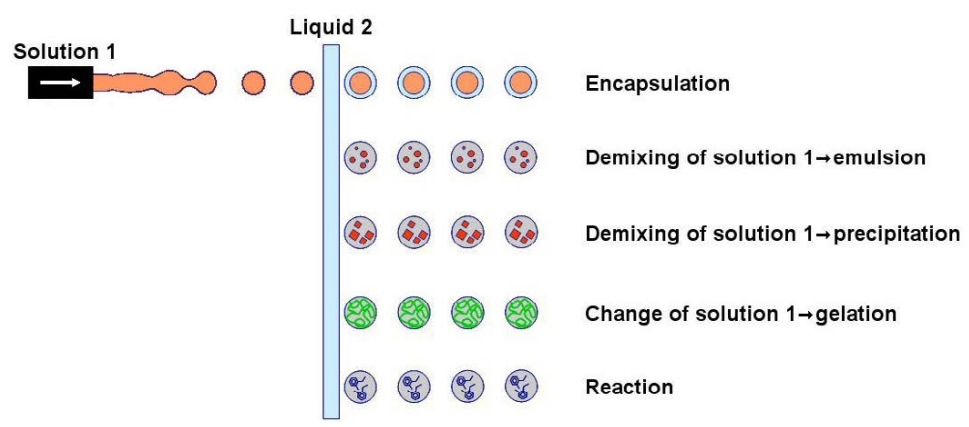

Figure 4.10: Principle of encapsulation and other effects which can occur. From demixing leading to emulsions or precipitation, towards gelation or even chemical reactions.

The processing technology has the following characteristics:

$\diamond$ Both material streams are separated, so the materials can be ejected under different processing conditions (e.g. temperature). In contrary to double nozzle encapsulators where both process streams are ejected though the same double nozzle at equal temperatures.

$\diamond$ The process can run continuously.

$\diamond$ It is a relatively mild process; sensitive emulsions are not affected.

$\diamond$ The monodisperse droplet size of the inkjet process results in monodisperse encapsulates with constant shell thicknesses, provided the liquid screen has a fixed thickness.

$\diamond$ Processing of the ejected droplet before and after impacting the screen is possible (e.g. drying, gas treatment).

$\diamond$ Viscous product streams can be used in both the inkjet and the screen.

$\diamond$ Using multiple liquid screens can enable multilayer shells.

In the following sub-paragraphs several encapsulates will be generated to investigate whether the features as described above really prove to be advantageous. Therefore an experimental setup has been designed. 


\subsubsection{Experimental setup}

An experimental setup is realized, build up out of modular aluminium profiles to achieve a system which can easily be adjusted (figure 4.11). The liquid screen is oriented vertically and can be oriented to maintain this specific position independent of screen settings. The printhead is mounted on a $x-y-z$ stage and is rotatable over two axes to allow exact setting of the impact point and angle. The impact can be visualised by two cameras using stroboscopic illumination to view both the impact as well as the exit point. To generate the liquid screen, a splash plate nozzle type is used where a jet impinges on the splash plate, resulting in a small but stable fluid screen, with a thickness which can be set to be in the same order as the droplet diameters used $[72,73]$.

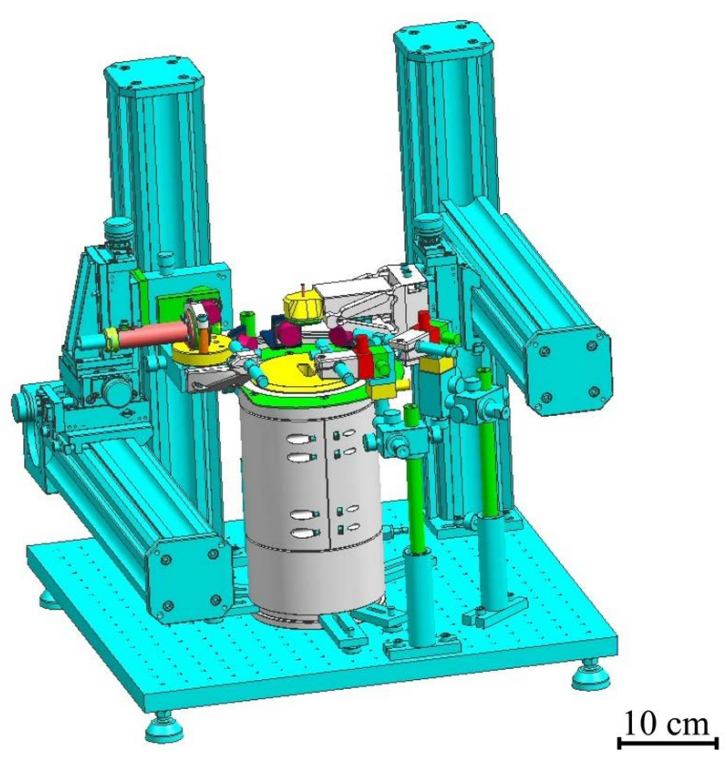

Figure 4.11: Representation of the experimental setup.

\subsubsection{Experimental results}

Three types of experiments are performed. Two related to encapsulation; first a hydrophobic shell material with an aqueous payload, and second, an oil based payload with an hydrophilic shell. A third experiment is preformed to achieve an in-flight reaction. 


\section{Experiment A: Encapsulation of aqueous solutions in a fat shell}

Hydrophobic shell materials such as fats or fatty acids are very interesting materials due to their water barrier properties. Several core-shell capsules are prepared by jetting droplets of different aqueous solutions through a liquid film of molten fat or fatty acid (figure 4.12).

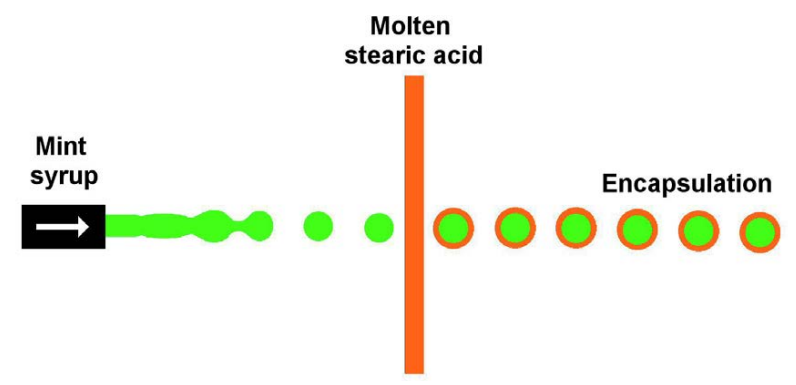

Figure 4.12: Schematic representation of experiment A.

Mint syrup is printed by means of the high viscosity printhead. The droplets are directed through a molten stearic acid screen. The stearic acid solidifies at room temperature to form the encapsulates. Figure 4.13 shows the core shell particles obtained. Depending on impact speed and screen temperature, either round particles with a diameter of $200 \mu \mathrm{m}$ are formed (low speed, high temperature) or extended particles can be created (high speed, low temperature).

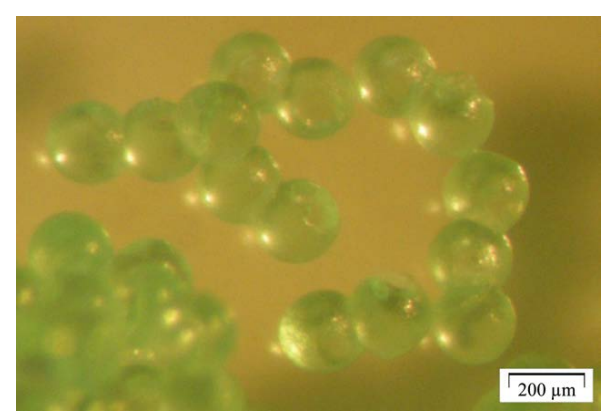

(a) Round particles are generated by jetting low speed particles through a high temperature screen.

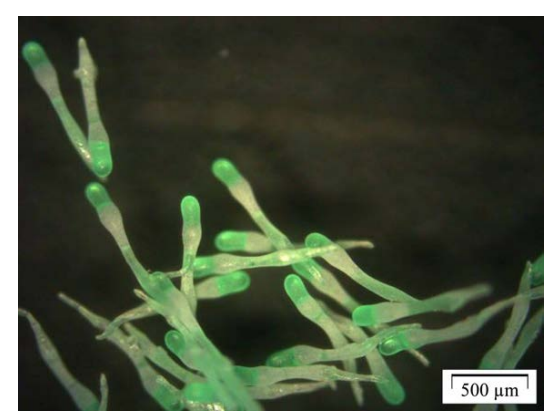

(b) Extended particles formed by using higher jet speeds through a screen held just above melting temperature.

Figure 4.13: Core shell particles of mint syrup in a stearic acid shell. 


\section{Experiment B: Encapsulation of oil}

Carrageenan $(3 \mathrm{wt} \%)$ is dissolved in demineralised water at $75^{\circ} \mathrm{C}$. A thin liquid film of this solution is produced, through which linseed oil droplets are jetted (figure 4.14). Immediately after encapsulation, the droplets are cooled, resulting in solidification of the carrageenan shell. Monodisperse microcapsules ( $280 \mu \mathrm{m}$ diameter) with a core of liquid oil and a soft polymer shell with a thickness of less than $10 \mu \mathrm{m}$ are obtained, corresponding to a core-to-shell volume ratio of around 85:15 (figure 4.15a). The thin polymer shell shows good mechanical stability when handling the microcapsules. The oil inside the capsule can be released by puncturing the shell with a pipette (figure 4.15b).

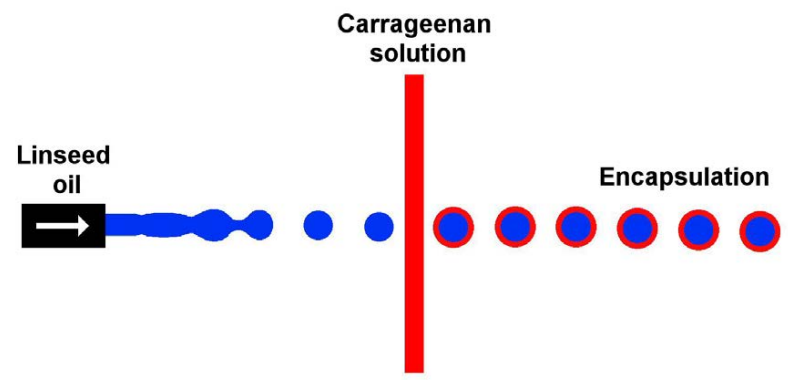

Figure 4.14: Schematic representation of experiment B.

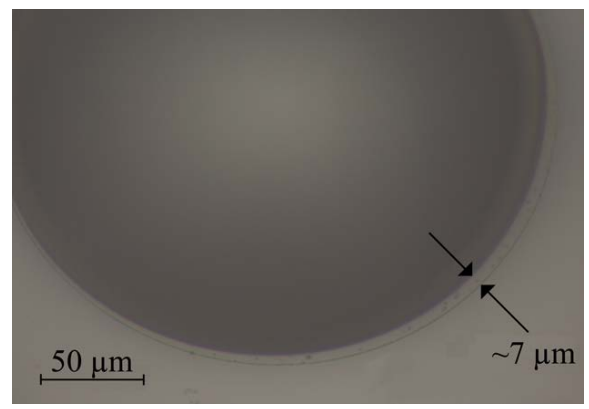

(a) $280 \mu \mathrm{m}$ diameter particle with a $7 \mu \mathrm{m}$ thick shell

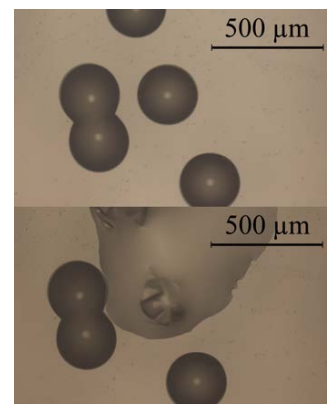

(b) Puncturing the shell with a pipette results in release of the core material.

Figure 4.15: Core shell particles of linseed oil in a carrageenan shell. 


\section{Experiment C: Monodisperse alginate microparticles}

Sodium alginate $(3 \mathrm{wt} \%)$ is dissolved in demineralised water. The resulting solution is printed through a liquid film consisting of an aqueous solution of $\mathrm{CaCl}_{2}$ (5 $\mathrm{wt} \%$ ), resulting in the formation of monodisperse alginate gel particles of $200 \mu \mathrm{m}$ diameter (figures 4.16 and 4.17). This methodology may also be used to encapsulate active ingredients by dispersing them in the printing liquid and collecting the gel particles. This way the ingredients are not surrounded by one single shell around the entire droplet like in experiment $\mathrm{A}$ and $\mathrm{B}$, but are fully embedded in the surrounding alginate gel.

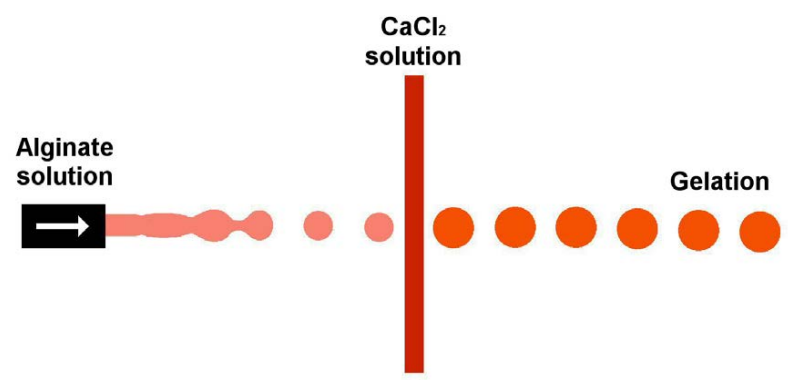

Figure 4.16: Schematic representation of experiment C.

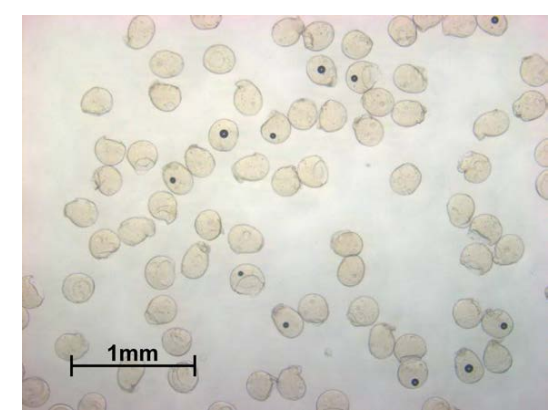

Figure 4.17: Generated alginate particles.

\subsubsection{Discussion}

Encapsulation of jetted droplets proved feasible. Both hydrophobic and hydrophilic materials can be encapsulated. Depending on screen and inkjet settings, different shell thicknesses can be obtained, but also the entire shape of the encapsulate can be 
controlled. At higher droplet speed it even becomes possible to add a specific amount of air in the encapsulate. The stroboscopic illumination is sufficient to visualise the impact of the droplet, the resulting sharp images prove stability of the process. Also in-flight reactions can be performed as shown with the third experiment. The described experiments show examples of possible situations; it is advised to study the effect of the different selectable parameters in more detail to fully exploit the concept.

\subsubsection{Alternative designs}

The encapsulation of very small $(\varnothing 50 \mu \mathrm{m})$ droplets becomes challenging. The smaller the droplets become the more problematic it becomes for the droplets to break through the liquid screen. One possible solution would be to increase the speed of the droplets. If the speed cannot be increased by a higher feed pressure of the inkjet another alternative is described in WO2009014432 [27]. A system is sketched where the droplets are aerodynamically accelerated to increase their speed (figure 4.18). The airflow is recycled to minimize the influence thereof on the liquid screen. If the aerodynamic design is chosen in such a way that the Stokes number $\left(S t=\frac{\rho_{p} D_{p} 2 U_{0}}{18 \mu_{f} L_{c}}\right)$ is low in the

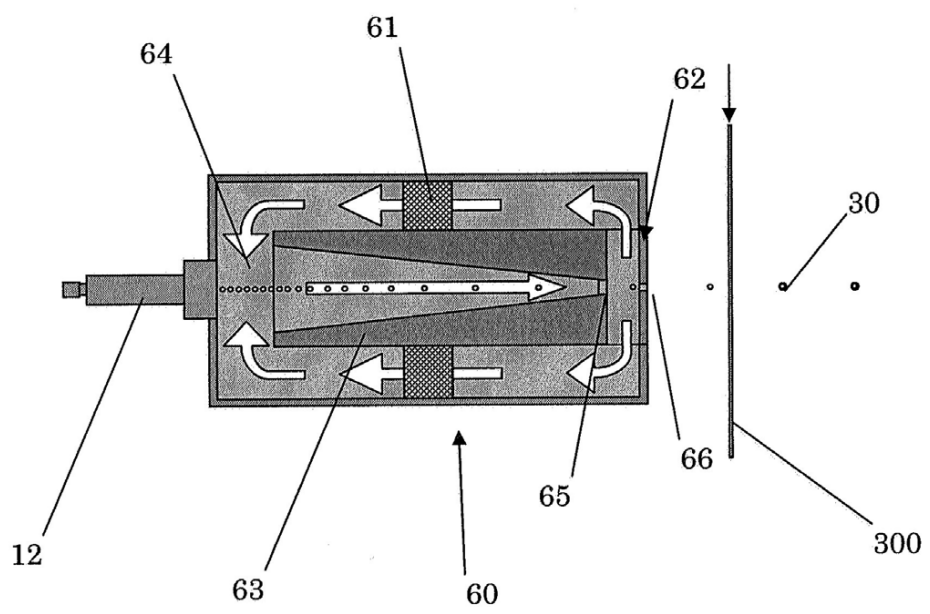

Figure 4.18: Multi component particle generating system, droplet acceleration [27]. A printhead (12) generates a stream of droplets. These droplets are accelerated through a converging passage (63), causing a high-speed airflow. The airflow is held in motion by fans (61). The high-speed droplets leave the accelerator through orifice 66 to collide with liquid screen 300 to form small encapsulates (30). A further in-depth description of all noted numbers can be found in the patent application. 
acceleration part, and high in the exit region, a focussing effect of the airflow can be designed equal to WO2009011583 [31] (described in more detail in the next chapter) to realize high speed droplets enabling puncture of the liquid screen. Here, $\rho_{p}$ represents the particle/droplet density, $D_{p}$ the particle/droplet diameter, $U_{0}$ the characteristic velocity, $\mu_{f}$ the fluid viscosity, in this case the viscosity of the air and $l_{c}$ the typical length scale.

Another solution is creating a thinner screen; unfortunately this is not straight forward. In WO2010090518 [28] a system is described using the ability to control the break-up process of a liquid screen (figure 4.19). As described in $[74,75]$ a perturbation in the screen will lead to thinner and thicker sections (figure 4.20), creating an extremely thin surface just before break-up. By synchronizing the printhead with the screen, the droplets can be set to collide with the thin section only, allowing small encapsulates to be generated.

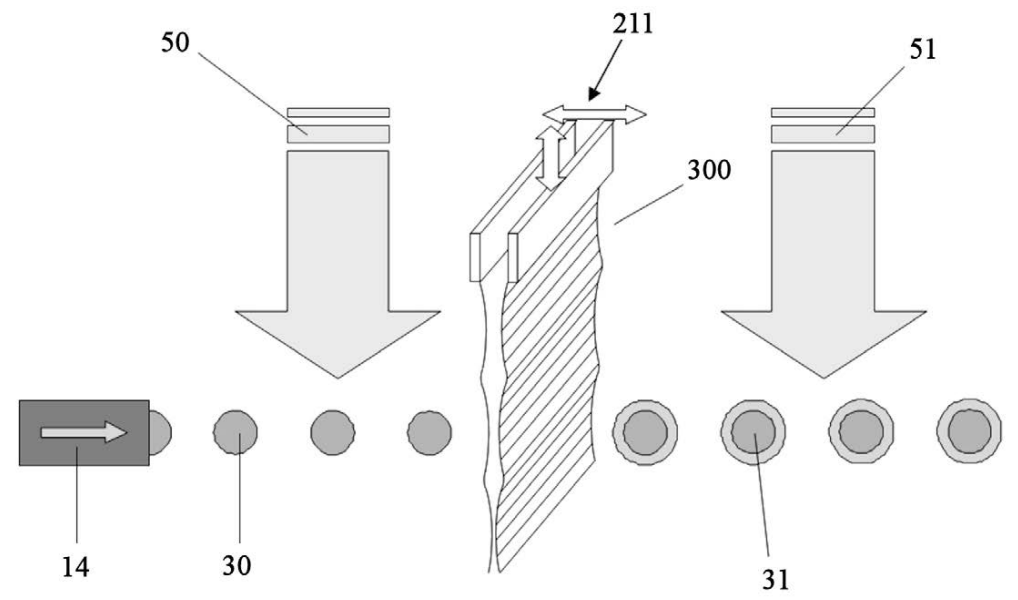

Figure 4.19: Multi component particle generating system, screen break-up mechanism [28]. A printhead (14) generates a stream of droplets (30). These droplets can be subjected to a drying or curing medium (50) before impacting on the liquid screen (300). After collision with the screen the droplets are coated, forming encapsulated droplets (31). These might be subjected to a drying or curing medium (51) to create the final particles. The screen is generated by a mechanism inducing a vibration (211) in the screen, causing the screen to break-up in a controlled manner, forming thicker and thinner sections. By exact timing of the printhead (14) and the modulation of the screen (211) the droplets can be set to collide with only the thin sections of the screen. 


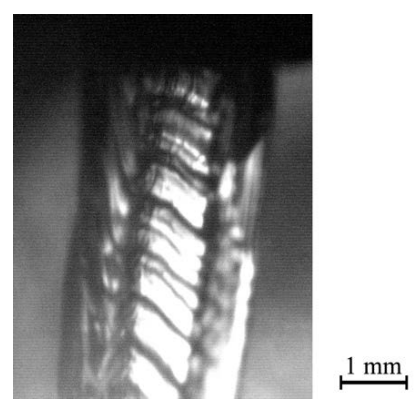

Figure 4.20: A stroboscopic image of liquid screen generated using a splash plate setup. The screen is agitated at a frequency of $20 \mathrm{kHz}$, showing controlled thickness variations in the middle of the screen. The thicker outer edges remain virtually unaffected.

Alternatively to printing the initial droplets one can also envision the demand for existing particles to be encapsulated. Various processes exist, however most result in a rather uncontrolled shell thickness. Equal to the screen print process in WO2010005302 [29] a process is sketched where particles are propelled through a liquid screen in a controlled manner, resulting in uniform encapsulates (figure 4.21).

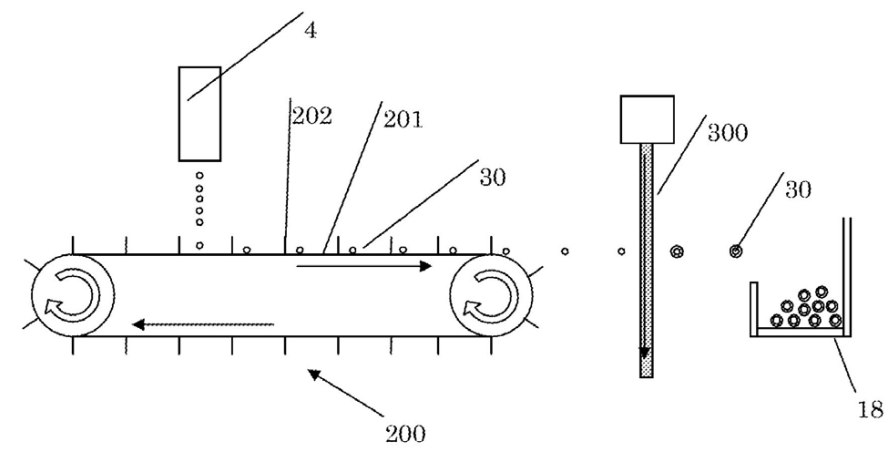

Figure 4.21: Multi component particle generating system, particle based [29]. Linear configuration: Powder particles are deposited on a conveyor belt (200) by dispenser 4. The particles (30) are subsequently thrown through liquid screen 300 creating encapsulates which are collected in gutter 18. A further in-depth description of all noted numbers can be found in the patent application. 
The patent also describes an embodiment how a scaled up process could be developed (figure 4.22). A circular liquid screen is generated, through which the particles are propelled. One can foresee the usage of the multi-nozzle printhead described in the previous paragraph in such a configuration, resulting in a system suitable for direct usage in conventional spray dryers.

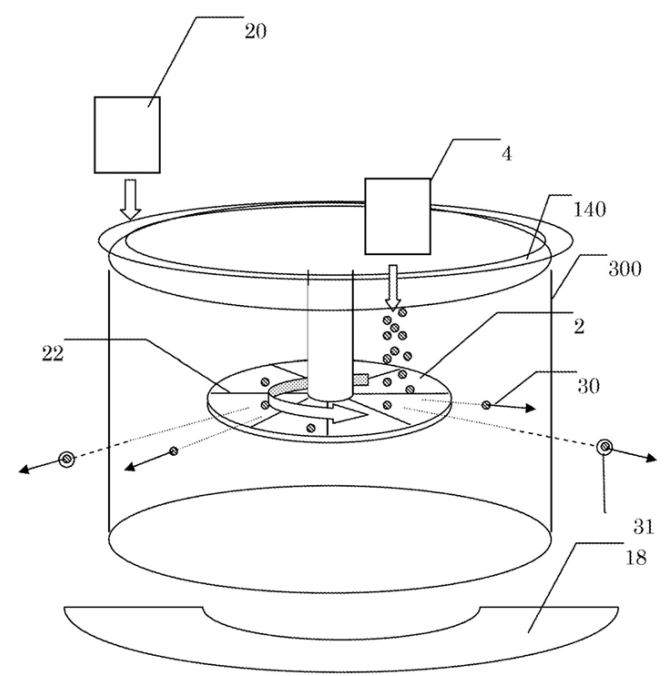

Figure 4.22: Multi component particle generating system, particle based [29]. Circular screen configuration: Particles (30) are deposited on a rotating disc (22) by dispenser 4. The disc propels the particles in a radial direction through a circular liquid screen (300) created by a liquid screen generator (20), causing encapsulates (31) to be created, which are collected in gutter 18. A further in-depth description of all noted numbers can be found in the patent application.

\subsection{Concluding remarks}

Altogether it can be concluded that the printhead initially developed for rapid manufacturing applications has a much broader range of application areas. In the field of powder processing the usage of the technology allows monodisperse powders to be created. The monodispese nature of the droplets results in increased powder properties, it also enables more exact dosing of the air needed for drying, resulting in an increase in energy efficiency. The printhead is able to process material feed containing higher concentrations of solids, resulting in an even higher overall system perfor- 
mance. The absence of fines results in a more economic process due to a reduction in the intensity of exhaust air filtration. The created powders reveal a different nature, compared to conventional agglomerated powders containing relatively large amounts of enclosed air. The resulting powders are spherical without air enclosures, resulting in high density powders. On the other hand, the process handles the materials with care, allowing processing of sensitive emulsions. The technology shows advantages in powder production; the control the printhead delivers goes further than only droplet size. The directionality and controlled speed of the droplets allows for the usage in an encapsulation process, propelling the generated droplets through a liquid screen. This new processing technology shows to be functional to create encapsulates both with a hydrophobic material contained in a hydrophilic shell, as well as the opposite, a hydrophilic payload inside a hydrophobic shell. Both process flows are generated separately, allowing for different initial parameters (temperature, speed), enabling creation of temperature sensitive payloads encapsulated in a for instance solidifying/heated wax. Also reactive systems can be combined due to the physical distance between the printhead and the liquid jet generator, in contrary to double nozzle encapsulators which frequently clog under these circumstances due to their integrated nature. The encapsulation process also shows opportunities for scale-up. The combination with a multi-nozzle printhead can be envisioned, allowing for direct usage in pilot scale spray dryers. Both applications promise great opportunities. The chapter described initial experiments to show the functionality of the principles. Both systems require further development and more extensive experiments to really prove their performance benefits. 


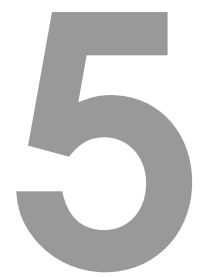

Metal printing ${ }^{1}$

In the previous chapters inkjet printing equipment has been described for the layer by layer manufacturing of fully functional end products. Processing of high quality materials was a minimum requirement for this purpose. Taking layer wise production to the next level would lead to the integration of electronic functionality and the deposition of conductive tracks in one single step. In a layer-wise production process there is no time for long curing or other multi-step metallization techniques. In this chapter two different approaches to metal deposition are discussed. The first, called pyrolitic metal printing, aims at printing highly conductive metal tracks in a one-step

\footnotetext{
${ }^{1}$ Published as:

$\diamond$ G. Oosterhuis, F. Feenstra, R. Houben, B. van der Vorst, L. Brouwers, F. Verhoeven, "Conductive metallic tracks by Pyrolytic printing, Innovative Production Machines and Systems", Fourth Innovative Production Machines and Systems Virtual Conference 7 - 10 (2008).

$\diamond$ F. Feenstra, R. Houben, L. Brouwers, P. Hofmann, J. Fischer-Buehner, U. Klotz, G. Wagner, P. Pierron, "Direct Metal Jetting", Innovative Production Machines and Systems, Fifth Innovative Production Machines and Systems Virtual Conference 1 - 4 (2009). In cooperation with Indutherm Erwaermungsanlagen $\mathrm{GmbH}$, Forschungsinstitut Edelmetalle \& Metallchemie, Reischauer $\mathrm{GmbH}$ and Ardeje SARL.
}

Based on:

$\diamond$ R.J. Houben, "Material jet system", (2007), WO 2007075084.

$\diamond$ R.J. Houben, G. Oosterhuis, "Method and apparatus for applying a material on a substrate", (2009), WO 2009011583. 
process. Pyrolytic printing starts with the generation of droplets from a solution of a precursor, guided by a nitrogen flow through a furnace evaporating the solvent, followed by pyrolysis of the precursor material, generating liquid metal particles. At the outlet of the furnace, aerodynamic focusing assures accurate deposition of the molten metal droplets, which yields $20 \mu \mathrm{m}$ wide conductive metal tracks directly onto the substrate. The technique provides a powerful alternative to existing printing techniques for metal track production. The second technique is developed to produce a rapid manufacturing system for highly detailed, custom-made metallic products by metal inkjet printing based on direct deposition of liquid metal. The technical feasibility and optimal process parameters of the individual processing steps of the metal printing technique are to be demonstrated and a prototype of the rapid manufacturing system, i.e. a molten droplet generation system, was built. The applicability of the developed manufacturing technique was assessed by specific demonstrators and product evaluation tests.

\subsection{Conductive metallic tracks by pyrolytic printing}

In the field of printed electronics depositing highly conductive tracks in a one-step process is considered as the ultimate goal, especially with minimal heat load onto the substrate. A process has been developed called pyrolytic metal printing, which has strong potential to achieve high conductivity with small track widths down to $20 \mu \mathrm{m}$. The method will be a powerful alternative to existing techniques to deposit conducting metal tracks.

\subsubsection{Overview of existing metal printing techniques}

There are many ways to produce conductive metal tracks of arbitrary geometry. Hence, new techniques should provide a distinct advantage with respect to the existing ones. Therefore, to facilitate comparison, a summary of the main existing metal printing techniques is given in Table 5.1. All direct deposition - or jetting techniques deposit the metal (or its precursor) directly at the desired location, thus affecting the surface at the location of the track printed. Deposition techniques like etching, screen printing and gravure affect the whole of the substrate body. This imposes boundary conditions on the whole of the surface, including the locations where no metal should be present. Such boundary condition may be: smooth geometry, chemical- and temperature resistance. Another distinction between direct deposition methods and other techniques is that in direct deposition, little material is wasted. In techniques that are based on material removal instead of deposition, material waste is 
inherent to the process. Jetting or direct-deposition has other application areas than techniques like etching or screen printing, and gravure. Therefore, the scope of the comparison is limited to direct deposition techniques. As can be seen in Table 5.1, each of the existing techniques implies the drawback of multiple process steps, material restrictions or thermal loads to the substrate. Spray pyrolysis eliminates such drawbacks but does not provide a means for accurate deposition. Pyrolytic metal printing combines the advantages of spray pyrolysis with a mono-disperse droplet generator (printhead) and aerodynamic focusing. This yields accurate and controlled deposition of the metal droplets. A method to realize such a printing process is described in more detail in the following subsections.

Table 5.1: Existing metal printing techniques.

\begin{tabular}{|l|l|}
\hline Technique & Description \\
\hline $\begin{array}{l}\text { Jetting silver nano- } \\
\text { particles suspension }\end{array}$ & $\begin{array}{l}\text { Jetting of silver nano-particles suspension, followed by } \\
\text { sintering [76]. }\end{array}$ \\
\hline $\begin{array}{l}\text { Spray deposition of } \\
\text { precursor solution or } \\
\text { nano-particle suspen- } \\
\text { sion }\end{array}$ & $\begin{array}{l}\text { Spray of } \mathrm{AgNO}_{3} \text {-solution, or silver nano-particle sus- } \\
\text { pension is deposited. Laser spot curing [77]. }\end{array}$ \\
\hline $\begin{array}{l}\text { Jetting a precursor fol- } \\
\text { lowed by metallization }\end{array}$ & $\begin{array}{l}\text { A precursor is jetted, metallization follows in the next } \\
\text { process step, by immersion in a solution that contains } \\
\text { metal ions, which causes a galvanic reaction [78]. }\end{array}$ \\
\hline $\begin{array}{l}\text { Direct jetting of molten } \\
\text { metal }\end{array}$ & $\begin{array}{l}\text { Inside the printhead, solid metal is melted and jetted } \\
\text { on the surface. Maximum operating temperature up to } \\
250^{\circ} \mathrm{C}, \text { only low melting point metals or alloys can be } \\
\text { used [79]. }\end{array}$ \\
\hline Spray pyrolysis & $\begin{array}{l}\text { A polydisperse spray of precursor solution is generated } \\
\text { and subsequently heated, causing solvent evaporation } \\
\text { and pyrolysis. Used to generate particles, no conductive } \\
\text { tracks. Not a jetting technique [80]. }\end{array}$ \\
\hline $\begin{array}{l}\text { Pyrolytic metal print- } \\
\text { ing (TNO) }\end{array}$ & $\begin{array}{l}\text { Monodisperse droplets of a precursor solution printed } \\
\text { by a printhead and subsequently heated, leading to } \\
\text { evaporation and pyrolysis. Molten metal droplets are } \\
\text { focused to form narrow tracks using aerodynamic fo- } \\
\text { cusing [81]. }\end{array}$ \\
\hline
\end{tabular}




\subsubsection{General process description}

The general process of pyrolytic metal printing can be described according to figure 5.1. As an example the pyrolisis of $\mathrm{AgNO}_{3}$ salt dissolved in water is described. $\mathrm{AgNO}_{3}$ acts as a precursor to create $\mathrm{Ag}$ tracks. Consecutively, the following process steps take place:

Droplet generation: The droplet is generated using a mono-disperse droplet generator. For the printing of 2D or 3D structures, a drop-on-demand printhead is required. For high-speed production of straight lines, continuous jetting may be used.

Droplet heat-up: Once the droplet is generated, the nitrogen flow carries it into the furnace where it is heated by infrared radiation. First, the absorbed heat causes a temperature increase.

Solvent evaporation: Once the droplet temperature approaches the boiling point of the solvent, the solvent will quickly evaporate, until only solid precursor particles remain.

Pyrolysis: Further heating will cause the precursor to melt $\left(212^{\circ} \mathrm{C}\right)$ after which it reaches the pyrolysis temperature of $\mathrm{AgNO}_{3}$ (above $440^{\circ} \mathrm{C}$ ). Then, the reaction $\mathrm{AgNO}_{3}(\mathrm{l}) \rightarrow \mathrm{Ag}(\mathrm{s})+\mathrm{NO}(\mathrm{g})+\mathrm{O}_{2}(\mathrm{~g})$ takes place, which yields solid silver.

Melting silver: Following the pyrolysis step, the solid silver particles are heated further, up to the melting point of silver $\left(962^{\circ} \mathrm{C}\right)$. Once molten, the droplet may be heated further until it leaves the furnace. In this phase, the heat input needs to be regulated in order to control the droplet temperature on impact, which highly influences the final track size and quality.

Focusing: Near the outlet of the furnace, the droplets may deviate from their initial course as a result of the preceding process steps. Therefore, aerodynamic focusing is required to (re-)direct the droplets in the center of the printed track. Focusing is achieved by accelerating the flow by means of a contraction at the end of the tube. During this acceleration of the gas flow, the droplet velocity also increases as a result of drag forces. The flow pattern is designed in such a way that there exists a transitional point from where the droplet motion is dominated by inertia instead of drag. The droplet trajectories exhibit a focal point, if the droplet velocity increases at least one order of magnitude in the converging part of the tube which has been confirmed by computational fluid dynamics (CFD) calculations. This way of focusing results in a deposition accuracy better than $5 \mu \mathrm{m}$. 


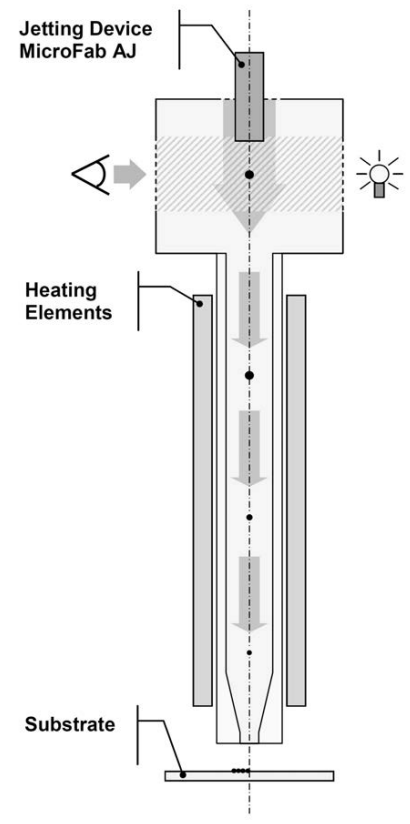

(a) Schematic system layout.

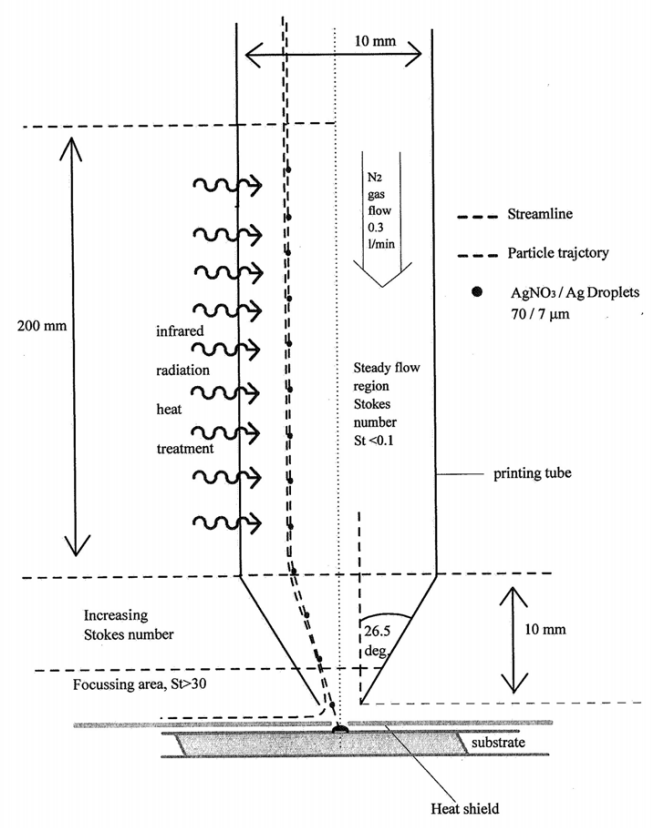

(b) Aerodynamic focussing in detail.

Figure 5.1: Schematic overview of the pyrolitic printing process [31].

\subsubsection{Experimental setup}

The printing setup comprises a drop-on-demand jetting device [79] that creates 30$100 \mu \mathrm{m}$ droplets of a $10-20 \% \mathrm{w} / \mathrm{w}$ (mass percentage) aqueous $\mathrm{AgNO}_{3}$ solution. The generated droplets pass through an inspection chamber followed by a ceramic tube furnace with a temperature up to $1500^{\circ} \mathrm{C}$. A nitrogen flow guides the droplets through the furnace. The substrate located several millimetres below the orifice is placed upon an $x-y$ stage to be able to generate $2 \mathrm{D}$ patterns.

\subsubsection{Experimental results}

Printing with this setup was successful up to the pyrolysis temperature of $\mathrm{AgNO}_{3}$. The dimensions and process conditions of the aerodynamic focusing of the test setup were optimized on the basis of CFD calculations. As a result, the aerodynamic focusing yielded a deposition accuracy of $5 \mu \mathrm{m}$. This is illustrated by a printed freestanding pillar of $20 \mu \mathrm{m}$ diameter and $2 \mathrm{~mm}$ height (figure 5.2a). The focusing results have been obtained at reduced furnace temperature. At this temperature, the salt 
was already molten but pyrolysis did not start yet. At higher furnace temperatures solid and molten silver droplets could be deposited in straight tracks that show good electrical conductivity (figure 5.2b) as well as high purity of the deposited silver (figure 5.3). The deposition of highly conductive silver tracks as well as the aerodynamic focusing were successful; however both aspects could not yet be achieved simultaneously. At temperatures necessary to achieve molten metal droplets, the focusing accuracy was reduced, resulting in a track width of approximately $300 \mu \mathrm{m}$, instead of the expected 20-50 $\mu \mathrm{m}$. Droplets break up into a poly-disperse spray with diameters ranging from $0.5-10 \mu \mathrm{m}$. As a result of both the smaller droplet size as well as the wider size distribution, the aerodynamic focusing accuracy decreases. Current efforts aim at understanding what phenomenon causes the break up of the droplets. Particularly challenging is the fact that the whole process takes place within a single-zone $1500^{\circ} \mathrm{C}$ furnace which excludes most of the existing inspection and measurement methods.

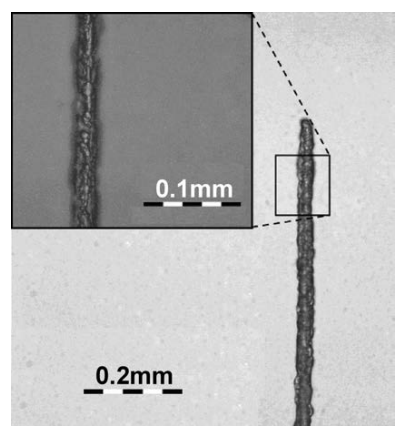

(a) Aerodynamic focusing; a free standing pillar could be printed of $2 \mathrm{~mm}$ high and 20 $\mu \mathrm{m}$ width.

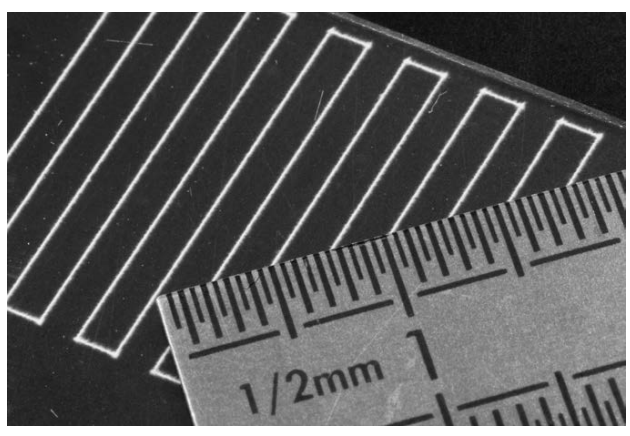

(b) Silver tracks with full metal-conductivity, printed on glass. The track width is $0.3 \pm 0.1 \mathrm{~mm}$.

Figure 5.2: Experimental printing results. 


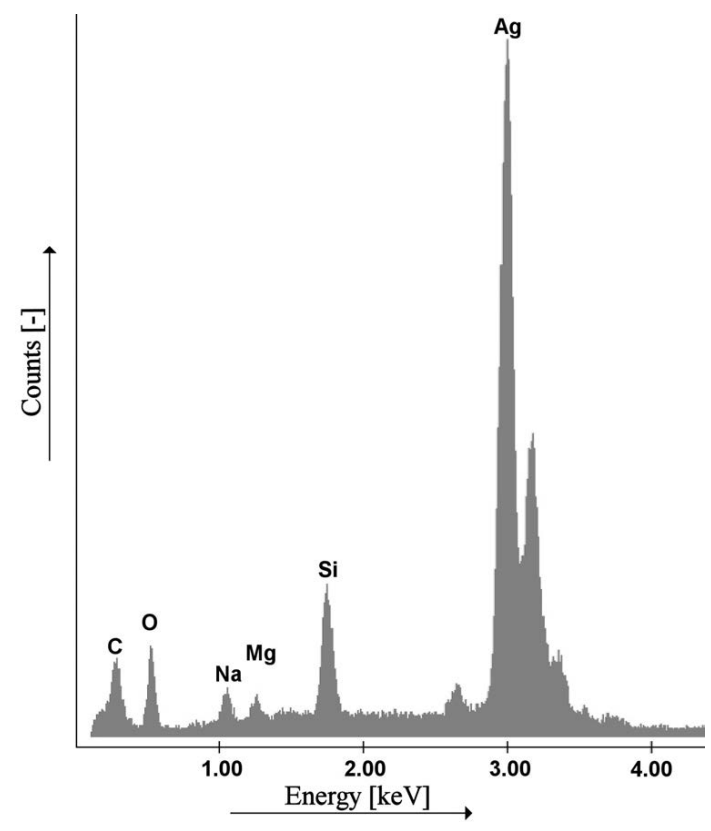

Figure 5.3: X-ray spectroscopy result of silver printed on glass. The deposited silver is very pure, the other peaks are attributed to the glass substrate.

\subsubsection{Concluding remarks}

Evaluation of several aspects reveals that pyrolytic metal printing provides a distinct advantage over existing techniques. Printing highly conductive metal tracks in a one-step process, with minimal heat load onto the substrate will become reality in the near future. The major challenge is to maintain the accurate focusing shown in figure 5.2a at elevated temperatures that yield molten silver droplets. Major future research topics will involve scaling up the process towards printing speeds required for industrial implementation.

\subsection{Direct Metal Jetting}

In the field of additive manufacturing the need is increasing for layer-wise production techniques able to produce (in acceptable time frames) parts of metal in sufficient quantities. In this field, four fundamentally different techniques already exploited in the market are potentially able to produce parts composed of either a sintered metal matrix or pure metal. These are three dimensional printing (3DP), selective 
laser sintering (SLS), electron beam melting (EBM) and laser engineered net shaping (LENS). All of them are powder technologies, where part of a bed of unbound powder is solidified by a gluing or laser process. These techniques are most suitable for robust 3D structures rather than fine structures such as decorations on coins or jewellery. In particular, the coarse size of the powder limits their accuracy.

In the field of injection moulding, powder technology is also used. With powder injection moulding (PIM) a process is developed where conventional injection moulding equipment can be used to create metal parts. An additional sintering phase afterwards is needed, similar to the post processing steps for the three dimensional printing process.

Direct metal jetting is a single-phase printing technology where individual droplets of molten metal are produced and deposited very precisely on predetermined positions (drop-on-demand). The development of the printhead as well as some preliminary results are described.

\subsubsection{Overview of metallic rapid manufacturing processes}

Several metallic rapid manufacturing processes exist [82]:

Three dimensional printing originates from the Massachusetts Institute of Technology and was developed in the mid-nineties of the last century. The process starts by depositing a layer of powder material at the top of a fabrication chamber. To accomplish this, a predetermined quantity of powder is first dispensed from a supply chamber by moving a piston upwards incrementally. The counter-rotating roller then distributes and compresses the powder at the top of the fabrication chamber. The multi-channel printhead subsequently deposits a liquid adhesive in a two dimensional pattern onto the layer of the powder which becomes bonded in the areas where the adhesive is deposited to form a layer of the object. This process is repeated until all the layers required to build the object are made. No support structures are needed since the powder deposited for earlier layers supports the new layer to be made. After completion the object is elevated and the powder surplus is brushed away. Three dimensional printing can be rather fast and allows multi-material output. A disadvantage of 3DP is that objects are porous, so surfaces must be post-treated to increase their durability (e.g. by infiltration with binder).

Selective laser sintering is similar to the three dimensional printing process, based on the principle of depositing layers of powder. However, the powder is not bonded by an adhesive, but by a laser beam that traces over the surface of 
the compacted powder to selectively melt and bond it to form a layer of the object. The fabrication chamber is maintained at a temperature just below the melting point of the powder, so that the heat induced by the laser only needs to elevate the temperature slightly to cause sintering of the powder. This process is repeated until all the layers required to build the object are made. No support structures are needed since the compacted powder deposited for earlier layers supports the new layer to be made. After completion the object is elevated and the powder surplus is brushed away. Available materials include not only plastic, but also metallic powders. Material properties can be quite close to those of bulk materials. Current developments are in the area of titanium and precious metals.

Electron beam melting is very similar to selective laser sintering. Instead of a laser source an electron beam gun is used for selectively heating the powder bed. The electron beam needs to be shielded because of radiation of gamma rays and the system must operate in a vacuum chamber. At the moment only steel and titanium alloys can be processed. Further, the process is limited to conductive metal powders. Fabricated objects are solid and need finishing.

Laser engineered net shaping is a technology in which an object is made from metal powder but different from selective laser sintering. A high power laser is used to melt metal powder supplied coaxially to the focus of the laser beam through a deposition head. The laser beam travels through the centre of the head and is focused to a small spot by a lens system. The $x-y$ table is moved in raster fashion to fabricate each layer of the object. The head is moved up vertically as each layer is completed. Metal powders are delivered and distributed around the circumference of the head either by gravity, or by using a pressurized carrier gas. An inert shroud gas is often used to shield the melt pool from atmospheric oxygen for better control of properties, and to promote layer by layer adhesion by providing better surface wetting. The technology is also known as laser cladding.

The direct metal printing process allows the use of materials such as stainless steel, copper, aluminium, etc. The material composition can be changed dynamically and continuously, leading to objects with properties which can not be made using conventional fabrication methods.

The metal printing technology promoted in this paragraph is not commercially available for rapid manufacturing purposes. This technique uses a precisely controlled stream of molten metal droplets. The metal is melted in a crucible which also 
contains a droplet generator and an orifice. The molten material runs into the orifice in the bottom of the crucible where it is fired by a piezo crystal. By doing so, a dropon-demand molten metal printer is realised. The substrate is moved by an x-y table which enables the printing of metal patterns. A schematic overview of the metalprint system is given in figure 5.4.

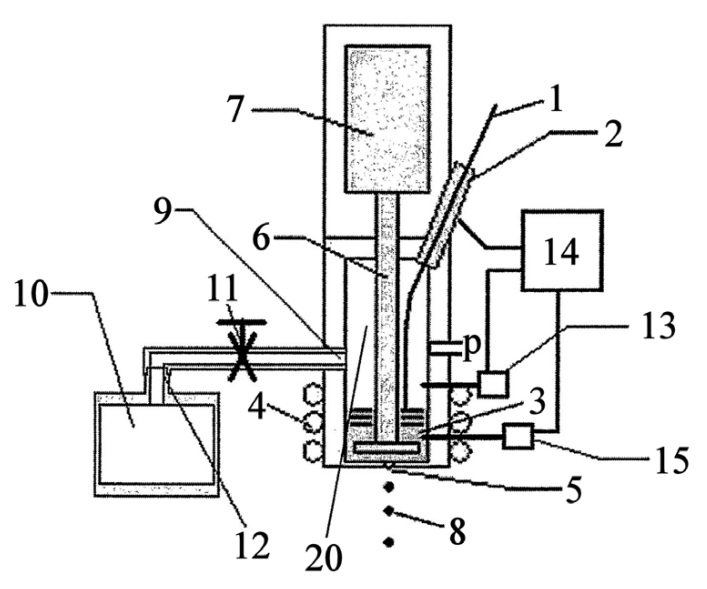

Figure 5.4: Schematic overview of the metalprint process [32]. A printhead (20) is filled with a small amount of metal (3) which is heated by means of heating elements (4). The metal is ejected through nozzle 5 to generate droplets (8) by means of a pressure pulse generated by piezo element 7 driving a focussing member (6) close to the nozzle. The system is equipped with a wire feed mechanism feeding metal wire (1) through a sealing mechanism (2). The system is regulated by a controller (14) measuring the liquid level in the printhead by means of sensors 13 and 15 to actively control the feeding of wire 1 . The system also comprises an overflow vessel (10) to periodically allow floating debris to be removed from the system through an overflow passage (9).

Advantages of the metal printing process are the potentially high material deposition rate $(0.6-2.5 \mathrm{~kg} / \mathrm{hr}$ dependent on the density of the material), the ability of multi-material use and the relatively low heat influx on the substrate material onto which the metal is printed. The technique essentially enables the production of full metallic products and alternatively, printing of metal on a non-conductive substrate to provide local conductivity while combining high accuracy with high material flexibility and throughput. Typical areas of application of full metallic products are jewellery (one-of-a-kind products) and automotive/aerospace (spare/overhauled) parts. Typical 
"conductive" applications are in electronics (MEMS, sensors, soldering, actuators, electronic hardware components, printed circuit boards of a cellular phone, custom made antennas). As compared to other manufacturing techniques for metal parts, 3D metal-printing is clearly the only one with the potential to evolve into a widely applicable and flexible manufacturing technique for many sectors in the coming years.

\subsubsection{Process description / experimental setup}

The general process of metal printing can be described according to figure 5.4. As an example the printing of Feingold is described. Consecutively, the following process steps take place:

Droplet heat-up: To melt and print the metal, a highly accurate, high temperature system is required up to a working temperature of $1140^{\circ} \mathrm{C}$. The induction principle is optimal for melting metals. The crucible is positioned in the core of the induction coil so that a steady temperature can directly be generated in the melting material. Because of the strong magnetic field the liquid metal will be mixed intensively. The so-called inductive bath movement makes the production of pre-alloys unnecessary.

Droplet generation: The droplet is generated using a piezo-driven ceramic agitation that causes a shock wave in the melt pool of gold or other metal. The piezo waveform is set in order to eject one single droplet from a $\emptyset 70 \mu \mathrm{m}$ orifice in a ceramic plate. For the printhead driving, a pulse generator and an amplifier are used to apply a voltage to the piezoelectric element. It allows the user to adjust the waveform that will be applied.

Droplet deposition: The printhead is fixed on a manual z-axis to adjust the distance between the substrate and the nozzle depending on substrate thickness (up to $200 \mathrm{~mm}$ ). Two cross-moving $\mathrm{x}$ and $\mathrm{y}$ stages are mounted underneath the printhead to allow printing of test products. Software was developed to control the motion of the respective axes and to synchronize the droplet ejection to the motion of the substrate. This combination allows the user to print a bitmap file.

Substrate: As a substrate, copper plates were used, which could be easily analysed by microscopy. 


\subsubsection{Experimental results}

With the experimental system several tests were performed. A picture of the experimental system in operation is given in figure 5.5. As a function of various parame-

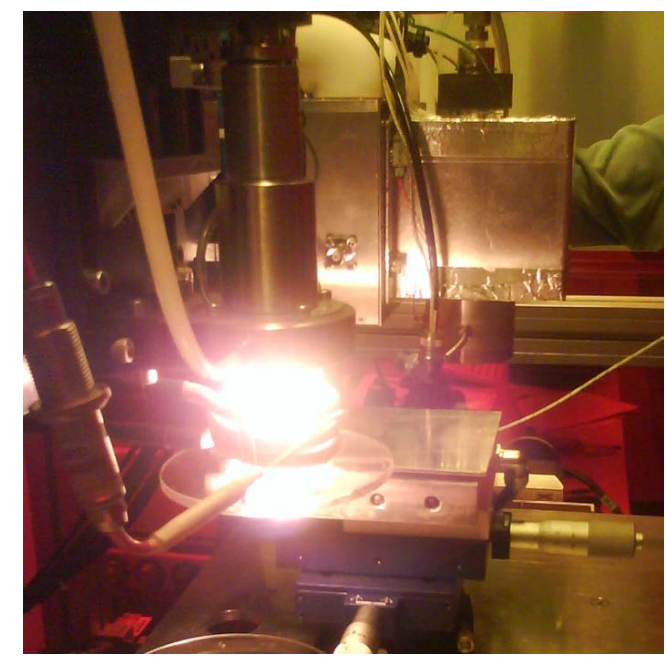

Figure 5.5: Metalprint experimental system in operation.

ters, the first test was to produce individual molten droplets of material to investigate the capabilities of the printhead itself. Due to the fact that a jetting principle was applied, drop-on-demand deposition of droplets was achieved, see figure 5.6a. Once the printhead was working properly, the software for $x-y$ movement of the platform was used to produce $2 \mathrm{D}$ printed bitmaps of Feingold, figure 5.6b. Also some trials

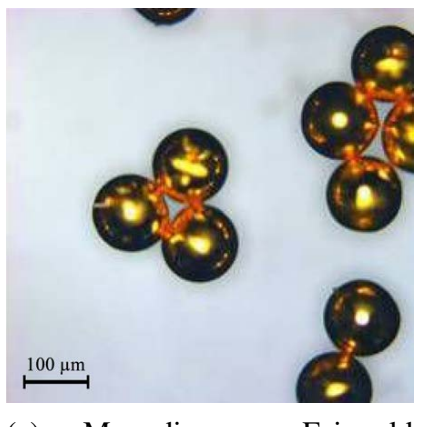

(a) Monodisperse Feingold droplets $\varnothing 120 \mu \mathrm{m}$.

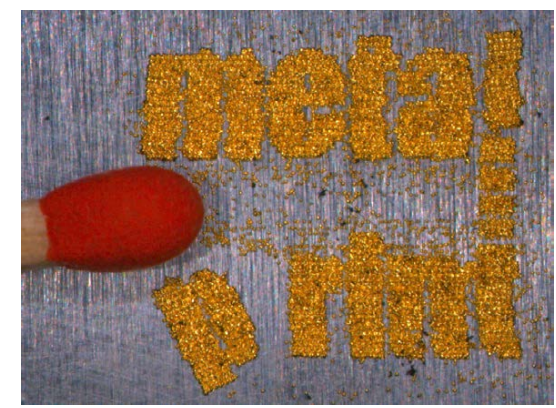

(b) Metalprinted bitmap of Feingold.

Figure 5.6: Experimental printing results. 
were made to produce 3D extruded piles of Feingold, figure 5.7a. Products were evaluated for part quality and micro-structure. For the application of a real functional end product realized by means of rapid manufacturing, it is necessary to have a good micro-structure, without any porosity and having small grains. The micro-structure of the printed 3D towers was assessed and results are given in figure $5.7 \mathrm{~b}$.

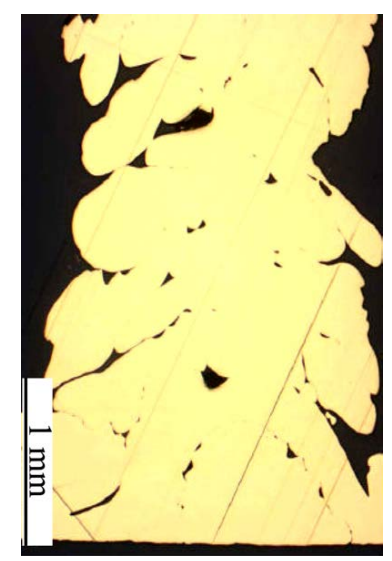

(a) Metallographic cross section.

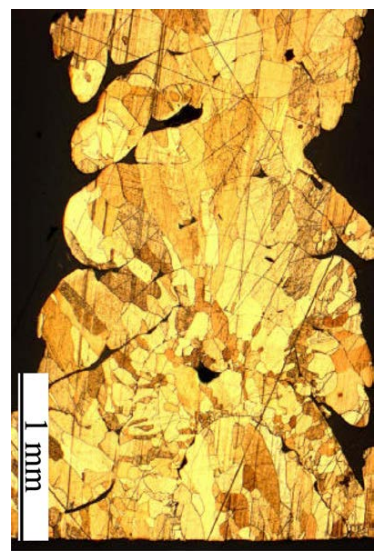

(b) Etched micro-structure.

Figure 5.7: Micro spectroscopy result of Feingold pillars.

The cross-sectional view of the vertical structure indicates that depending on the ambient temperature, some grain boundaries as well as welding lines can be discerned. The piles were created by subsequently adding droplets. The investigation focusses on the central part of the piles to determine whether the individual droplets remain or the grains span over the original droplet interfaces. A dense structure is found with nearly no porosity in the center. This indicates that the metal printing process indeed is capable to produce parts with a fine micro structure. Large 3D structures could not be made due to the limitation of the setup which had no thermal conditioning of the substrate. A limited droplet placement accuracy is observed due to the limited drop speed (estimated to be around $0.5 \mathrm{~m} / \mathrm{s}$ ). A higher droplet speed combined with a thermally well conditioned substrate would probably enable printing of larger dense structures, maintaining the fine micro-structure.

\subsubsection{Concluding remarks}

The metalprint drop-on-demand printer has demonstrated to be able to print bitmaps and pictures on substrates, as well as 3D columns of limited height. A basis for a 
successful metal printing technique has been provided. The system can work in a drop-on-demand mode, delivering one molten droplet of gold per impulse. Experiments with gold show a defect-free, recrystallised micro-structure, which is a prerequisite for making solid parts. Further experiments with a modified $x-y$ stage are necessary and possible to make additional $2 \mathrm{D}$ demonstrators and eye-catching products. If a substrate heater or oven is installed to the machine which keeps the sample at high temperature, it is possible to get good adhesion among layers. The frequency of jetting droplets is limited to 500 droplets per second. Currently, printing is limited to about $1 \mathrm{~cm}^{2}$ per 3 minutes fully covered surface, however this has not yet been optimised. This can all be attributed to the nature of stop-and-go printing rather than printing on the fly. Accuracy of deposition is about $0.1 \mathrm{~mm}$. This is lower than originally planned. Stacking of droplets to make 3D or vertically extruded columns was demonstrated to be working, however, was not yet intensively tested. Only stacked single droplets piles could be made. 2D bitmaps of pictures could be printed on flat substrates and indicate the ability to print any bitmap in a drop-on-demand mode. The setting of the resolution can be varied with the software. A point of concern is the detailing; due to the droplet size being in the area of $100 \mu \mathrm{m}$, very small details cannot be made. Experiments showed that faster printing enables complete towers with a fine micro-structure to be produced, so it is a matter of fine-tuning the droplet frequency and the temperature to improve the quality of the 3D-printed part. 


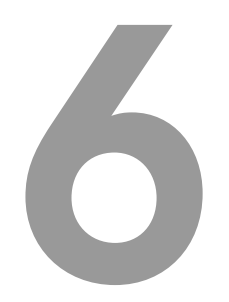

\section{D printing; from layer formation to three dimensional objects ${ }^{1}$}

Rapid prototyping has evolved to rapid manufacturing. A dot-matrix printer has evolved to full colour inkjet. In this chapter, the implementation of the inkjet technology developed in this theses, is described to form end-products. Combining multiple printheads in one single setup, to allow multi-material products to be created, requires real-time droplet control. New software is needed since current design tools do not allow multiple material properties to be defined, let alone the definition of graded structures. A demonstrator part is generated showing the capabilities of the system, and applications of the printing technology are described.

\footnotetext{
${ }^{1}$ Published as:

$\diamond$ R.J. Houben, L.A.M. Brouwers, A. Rijfers, M.A. Willemse , "Inkjet printing for Rapid Manufacturing: Printing towards the future", Innovative Production Machines and Systems, Fifth Innovative Production Machines and Systems Virtual Conference 19 - 22 (2009).
} 


\subsection{Printing functional material}

The current speed of development and the growing demand for customisation raises a demand for new manufacturing processes. Efficient usage of raw materials becomes more and more important. Inkjet technology if made applicable for manufacturing purposes could result in a major breakthrough, not only because of its ability to selectively deposit only the desired material; it even enables the manufacturing of multimaterial product. It opens a world to the generation of graded products. A world where products can be build-up of multiple materials which gradually change from one material to the other, thus enabling totally new applications and more efficient product design.

Current research activities focus on developing new processes and improving existing rapid manufacturing techniques. Several existing processes already use inkjet technology to manufacture layered products. Currently available printheads are able to process liquids with a maximum viscosity of $30 \mathrm{mPa} \cdot \mathrm{s}$ [12]. To generate functional end products this viscosity appears to be the limiting factor. A printhead has been developed (see chapter 2) that is able to process high-tech materials with a viscosity up to $500 \mathrm{mPa} \cdot \mathrm{s}$ at ambient temperature, overcoming this major drawback. In general, the advantage of using these high viscosity inks is that the material properties of the end product will be better, which enables the manufacturing of functional products.

\subsection{General process description}

The machine developed contains three printheads (see figure 6.1) that sequentially deposit various UV curable materials on a moving substrate. This occurs, depending in part on the viscosity of the liquid, at a frequency between 10 and $140 \mathrm{kHz}$, implying generation of 140000 droplets per second. The stage moves at a speed up to $6 \mathrm{~m} / \mathrm{s}$ and the flow rate of the printheads can be adjusted from 0.5 to $4.0 \mathrm{ml} / \mathrm{min}$. These speeds can be chosen according to the application. The standard printheads work at temperatures of $20^{\circ} \mathrm{C}$ to $80^{\circ} \mathrm{C}$. A modified printhead can even operate at temperatures of up to $350^{\circ} \mathrm{C}$. The higher the temperature, the lower the viscosity of the liquid, which can facilitate the printing process. The volume of a droplet can be selected, depending on the settings used, as low as 30 picolitres up to nanolitres. Figure 6.2 shows the printhead that is used to process the high-viscous material [17]. 


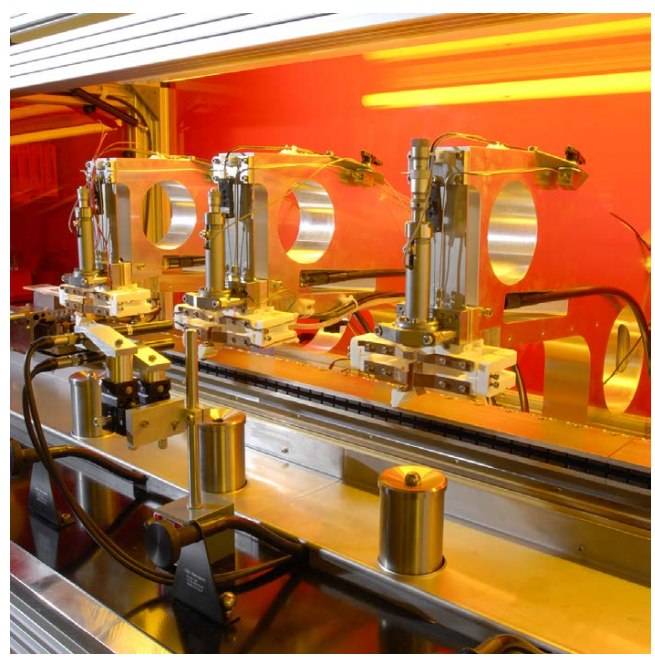

Figure 6.1: Three printheads combined in one test rig.

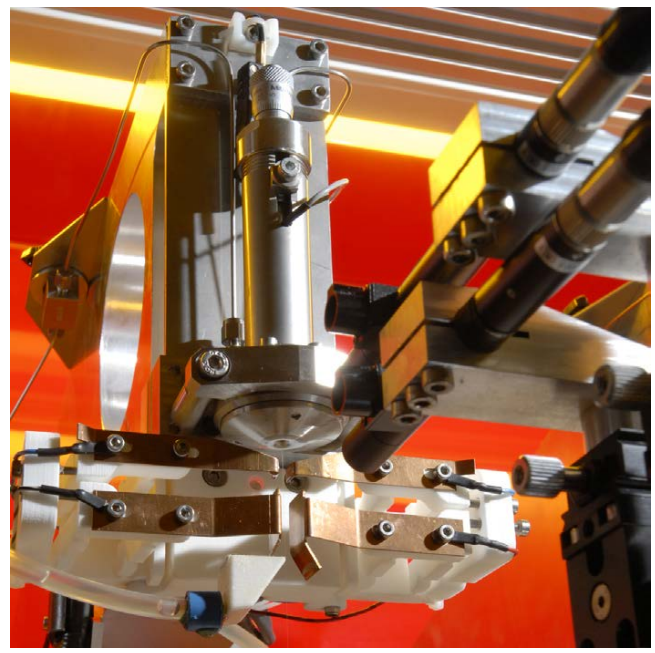

Figure 6.2: The high viscosity printhead with deflection unit. 


\subsubsection{Droplet generation}

The printhead is based on the principle of Rayleigh jet break-up [83]. A continuous jet is generated by forcing fluid through a nozzle. This jet is disturbed by means of piezo-vibration. By correct selection of frequency and intensity of the perturbation, the break-up of the fluid jet can be controlled, enabling control over drop formation. The printhead is designed to be serviceable and adjustable. The aim is to be able to be totally independent of the viscosity of the material to be processed in contrary to conventional inkjet printheads and to eliminate the need of material adaptation before printing a specific material.

\subsubsection{Droplet selection}

As mentioned above a continuous stream of droplets is formed. However, selective printing is required to be able to print a pattern. Thus a droplet selection system is necessary. An electrostatic deflection unit is applied. It consists of a charging electrode (see figure 6.3; top electrode pair) which can charge selected droplets and a second electrode pair over which a high voltage is set, which creates an electrostatic field (see figure 6.3; bottom electrode pair). The droplets are passed through this field; charged droplets are pulled out of their initial path and directed towards a gutter for recycling. Non-charged droplets follow their initial path and reach their desired position on the substrate or product [14]. For this system to be operational

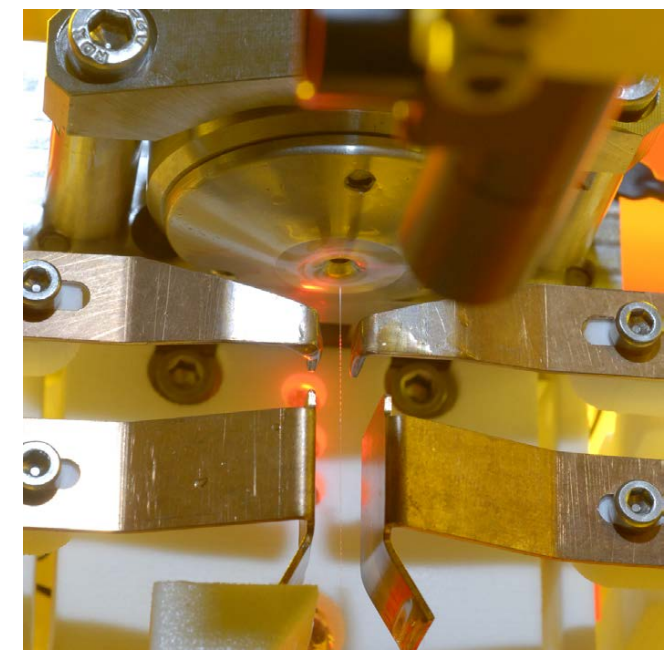

Figure 6.3: Close-up of the jet and deflection unit. 
the printed material has to have a certain conductivity to be chargeable. Conventional low viscosity continuous inkjet printers also use a slightly conductive ink; this can easily be achieved due to the solvents used. The ability to print high viscous materials makes the use of solvents unnecessary, enabling the use of solvent free materials. Here, however, an issue occurs to create a solvent free material which has sufficient electrical conductivity to make the charging of the droplets possible. Several solutions have been investigated resulting in a material combination delivering enough conductivity without the need for excessive material adjustment [23], described in more detail in chapter 3 of this thesis.

\subsubsection{Product build-up}

The test rig is developed to work with stationary printheads. The product is being passed underneath the printheads again and again during build-up. With each pass, a line is selectively printed. Real-time electronics control drop position. The product material can be cured each pass by UV-exposure with a UV-source mounted on one end of the stage. Supplying the system with 3D multi material files with gradients posed a problem since most 3D software only describes the outside surface of a part. Software has been developed to define the inside of a product. The developed software is also capable of applying gradients. Combined printing with all printheads results in a three dimensional product. A transparent base material is used and two spirals are added slowly fading away. Figure 6.4 shows the final product.

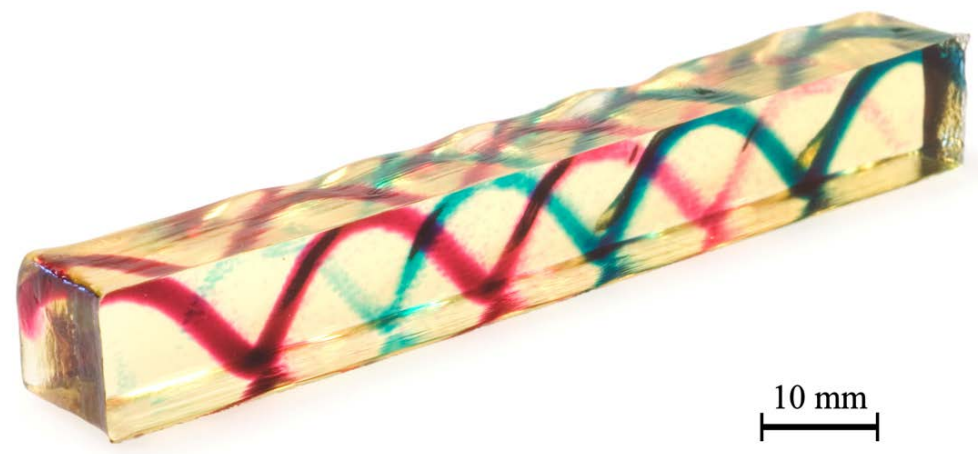

Figure 6.4: Sample product with multiple gradients. 


\subsection{Applications}

One application is the manufacture of custom-made implants of biocompatible and bio-degradable material. The ultimate aim was to make a jaw implant from a material in which the body's own bone can re-grow. The implanted material will then be broken down by the body. To stimulate cell growth in the implant, growth promotors can be added selectively. Depending on the required cell type and the extent of growth desired a measured quantity of growth stimulator should be added at each position in the implant.

Next to three-dimensional products the print technology can also be used to print two-dimensional patterns. Due to it's flexibility in material and high line speed, the printhead can be used in a high-speed production setup for deposition of specific materials in very small amounts.

Another application in a total different area makes use of another unique feature of the system. The printhead is able to process high viscous material into monodisperse droplets. Drying these droplets yield energy as well as product property benefits as described in more detail in chapter 4 [26].

\subsection{Concluding remarks}

A new printing process has been developed enabling direct production of end products. Combining multiple materials into one single product opens new opportunities. This process is used in one of the first systems to generate gradients in material properties within one product. This ability sets the need for a radical new way of product development and design. Until now only nature was able to generate these structures. Now production technology is starting to catch up. 


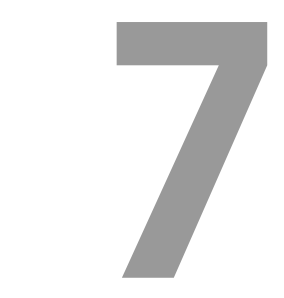

\section{Epilogue}

What can be expected in the future, what are the challenges? Which drivers will cause additive manufacturing to reach a breakthrough and start the third industrial revolution [84]? The technology is already finding its way into niche applications like personalized products such as hearing aids and dental applications. For the technology to broaden its application area the range of applicable materials needs to be extended and the manufacturing speed needs to increase. To achieve this, new production configurations are necessary taking the step from batch-to continuous production systems as well as new deposition systems as described in this thesis. But the technology should also be available for the designers who need to free their mind to allow them to design new generations of products utilizing the benefits of the technology, creating products which cannot be made using conventional production machines.

Inkjet technology has been promising for years in industrial applications. For the technology to fulfil the expectations, the development of the technology should not be led by the history of the companies involved, but by the application the technology is meant for. Introducing disposable nozzle arrays might lead to a different approach with respect to reliability. Further integration of microfluidic structures inside the deposition module can revolutionize the way functional printing is utilized nowadays. 


\subsection{Additive manufacturing}

Today the world of additive manufacturing behaves similar to the development of digital printing in the past. Will everybody have a 3D printer at home, or will there be centralized 3D manufacturing facilities? The answer is probably as dual as the world of inkjet. Presently, additive manufacturing is not mature yet. The technology available for home applications is of (too) low quality and too expensive, and the industrial processes are only applicable for niche applications. So how can we boost the development for the technology to flourish?

\subsubsection{Which market drivers exist?}

Some people are convinced that everybody will end up with a 3D printer at home. And this will probably be true depending on the definition of a 3D printer. A layerwise production machine that can create virtually everything is doubtful. But a system which can create specific products on-demand can become reality. One can wonder for what reason people would like to have a 3D printer? Which characteristic of the system is most favourable?

To personalize a product? Make a product to suit a specific person. E.g. personalized shoes, fitting perfectly or medical prostheses made specific to fit the person.

To customize a product? Create a product to a customer's specific liking. Where a laptop started out as a gray small computer, nowadays people buy them in all kind of colours.

To create a product on-demand? Instead of having a lot of products on stock, print the product you need at that specific moment.

To create a product which currently cannot be fabricated? Utilizing the advantages of additive manufacturing to the fullest creating a graded integrated multi material product. The technology is pushed forward through its ability to produce products which cannot be made using conventional production techniques.

To personalize a product? Looking at current applications of additive manufacturing, the first routes seems to be taken. In personalized markets (dental, hearing aids, etc.), where current production is still labour intensive, the ability of additive manufacturing to create personalized products becomes cost effective already. 
To customize a product? If one would look far forward in the future to the point where a $3 \mathrm{D}$ printer is available in every home, capable of printing virtually everything, this would have a disruptive effect on our current way of life and on the economic system worldwide. What would have value, if everything you would like to buy can be printed at home? What is the need for freight traffic if only raw materials need to be transported, in stead of end products? But probably even more challenging, how do people differentiate from each other if everybody can produce the product one currently would like to have (since his neighbour has one). Would this really lead to a more customized and personalized world or would men proof to be more of a herd animal and print out the same product since it is "cool"? This might be a great topic for a sociologist to pick up.

To create a product on-demand? Nowadays a lot of products are manufactured; the variety in products keeps growing. Since most of these products are manufactured in large quantities in low wage countries, large freights are transported around the world, to be stocked in warehouses until potentially sold to a customer. Unfortunately not all products are sold, and are either sold below value or even discarded. Closely linked to creating a product on-demand, is creating a product on-site, making large stocks obsolete and significantly reducing necessary freight transport. If material scarcity increases and overproduction becomes more costly, on-demand production becomes interesting. This might be accelerated thinking about the flexibility of on-demand production, the time in-between different product series, or models could be significantly decreased, leading to a shorter time to market. When personalized or customized products cannot be made at home, but should be ordered in central manufacturing facilities, on-site production becomes of interest due to the shorter/cheaper shipping cost which might save industrial activity in Europe and other high wage counties. On-demand production can be of interest already, thinking about spare parts. Nowadays a lot of spare parts from all created products need to be on stock, with increased renewal speed of current designs the spare part list increases. Instead of stockpiling these parts, on-demand fabrication thereof is already interesting in specific areas.

To create a product which currently cannot be fabricated? A real break-through for additive manufacturing could be made if the last approach could be taken, here unfortunately a challenge occurs. To develop and design such a breakthrough application of the technology, current designers should be fully familiar with the technology and its possibilities and opportunities. Therefore, instead of teaching mechanical engineers to design parts for manufacturability, one should try to broaden their spectrum. 
All parts made by men already teach the boundaries given by current production technology. The form freedom given by layer-wise production supplies a huge opportunity, allowing far more function integration than currently applied in products. It is not straight forward for developers to unlearn the design rules they have been using for years, and use the benefits of current layer-wise technology in their designs. Let alone making designs using the opportunities of future generations of additive manufacturing processes, where it becomes possible to design non assembled multi material structures, or the paradigm shift when using graded material properties inside products. Therefore it all starts with education to supply future engineers with the right mindset to allow them to clear their minds to come to these breakthrough designs.

All of the above looks very promising, but there is always one primary aspect of crucial importance for market attractiveness; the cost price of the finished product in combination with its value. In relationship to additive manufacturing, a second aspect should not be forgotten, delivery time.

\subsubsection{What should be developed to support these drivers?}

Software tools to design the products: Instead of waiting for breakthrough designs showing the need for layer-wise manufacturing to drive the technology forward, we can help designers by making their future toolbox as easy to use as possible, to express their ideas and create shapes without any limitations. Allowing the next generation of multi material graded products to be designed.

Processing tools to deposit the required materials: Of crucial importance is the ability to create the products required, having the functionality necessary to fulfil the job intended. And this should be competitive compared to commonly used production technologies in terms of price and function. The huge step necessary is developing the deposition systems capable of depositing the required materials.

Speed: One aspect most of the time directly linked to production cost is production speed. Looking at current additive manufacturing processes it can be noticed that they are all upgraded prototyping machines, designed to fabricate small batches of products. For layer-wise production to evolve to a real production technology these steps have to be made with respect to process layout. Optimization of the usage of the deposition systems is a route for achieving competitive production speed. Shifting from batch towards continuous production configurations gives opportunities for making the step from products a day to products a minute (figure 7.1). 


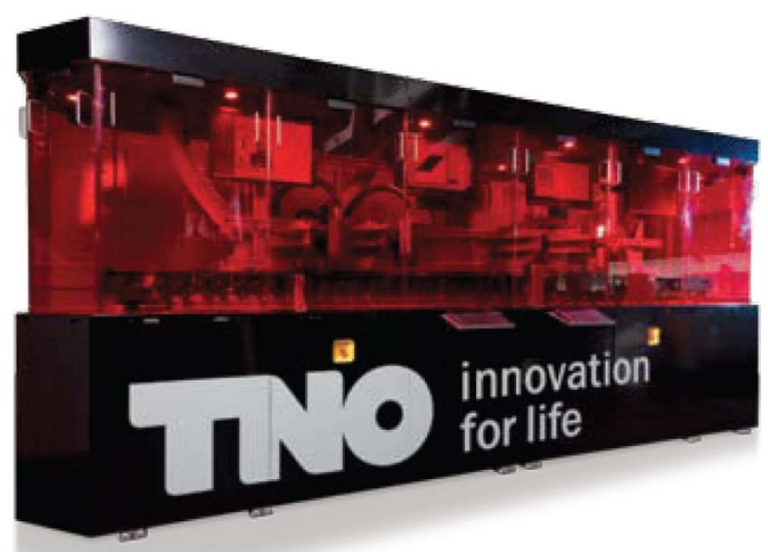

Figure 7.1: Additive manufacturing production machine. 100 carriages travel at high speed $(2 \mathrm{~m} / \mathrm{s})$ past stationary deposition units. Single pass deposition units supply the passing product with a full layer with each pass. The production carriages are connected with minimum space in between to reach optimal coverage of the deposition units. No time is lost by back and forth motions since all products follow a repeating circular path. Instead of building a 50x75x6 $\mathrm{mm}$ part in 1.5 hours this layout provides a platform to reach a finished part every 6 seconds (100 parts in 10 minutes) [85].

\subsection{Inkjet development}

For such a system to be used to its limits the inkjet technology described in this thesis should be further engineered towards a multi-nozzle print system capable of single pass deposition. This can be done conventionally by widening the system and adding a nozzle plate with multiple holes, analogue to the printhead described in chapter 4 . For accurate deposition using a continuous inkjet layout it is crucial for the nozzles to be identical (due to the relatively long throw distance, angle deviations are catastrophic). Instead of using laser drilled or spark eroded stainless steel nozzle plates, changing to semiconductor device fabrication technologies creating silicon nozzles can deliver whole new chances. Older generation device fabrication machines which cannot reach the resolution and accuracy needed for the latest generation microchips are more than accurate enough for the generation of nozzle arrays. 


\subsubsection{Disposable nozzle array as a route to industrial reliability}

Looking at implementation of inkjet technology in industrial applications, the technology has looked promising for years, however real breakthroughs have been missing so far. The described development route in this thesis might give possibilities. Most printheads used for industrial applications are derived from printheads designed for graphic applications. With these printheads the necessity exists to formulate the material in such a way that it can be processed through the printing system. Unfortunately, a lot of reformulating is necessary mostly not adding anything to the function the material needs to perform once deposited. It can be argued whether current dropon-demand systems commonly used in industrial graphics markets can be redesigned in such a way that they will be able to process a wider range of materials, or even be material independent. While this might be a necessity for the technology to flourish in industrial applications, and to become disruptive, as promised for years. Taking a totally different approach might be unavoidable.

A similar argumentation can be given for the route towards reliability. A natural development leads to a demand for more robust and reliable production systems when applied in industrial applications. If a relatively expensive multi-nozzle dropon-demand printhead is used in an industrial process one would like such a printhead operating as reliably as possible. When considering the trend towards single pass deposition, most of the time multiple printheads are used. So replacing a printhead array becomes a costly business. In most cases commercially available printheads are taken as a starting point, initially developed for a whole different application field. So also with respect to reliability one should make a deliberate choice which route to follow. It is probably one of the most far-reaching requirements one has to consider when developing a system utilizing inkjet technology, analogue to the current split-up in inkjet systems in home applications between disposable printheads (HP) and printheads designed to last the lifetime of the printer (Brother, Epson). Almost all high-performance printheads for industrial applications fall in the non-disposable category. The described route for generating printheads using microchip technology is currently adopted by multiple printhead manufactures, most of them looking for cheaper and better ways for the creation of their multi-nozzle drop-on-demand printheads. When developing a drop-on-demand system it is a logical choice to create a fully integrated device with a combination of fluidic channels and actuation mechanisms, leading to a relatively costly device to discard. If this technology would be used for continuous inkjet systems, the actuation mechanism can be built conventionally leaving only the nozzle array as a disposable part. Designing a sealed nozzle array with internal filtration might provide an alternative route to achieve a cost effective route to a disposable system for industrial applications (figure 7.2). 


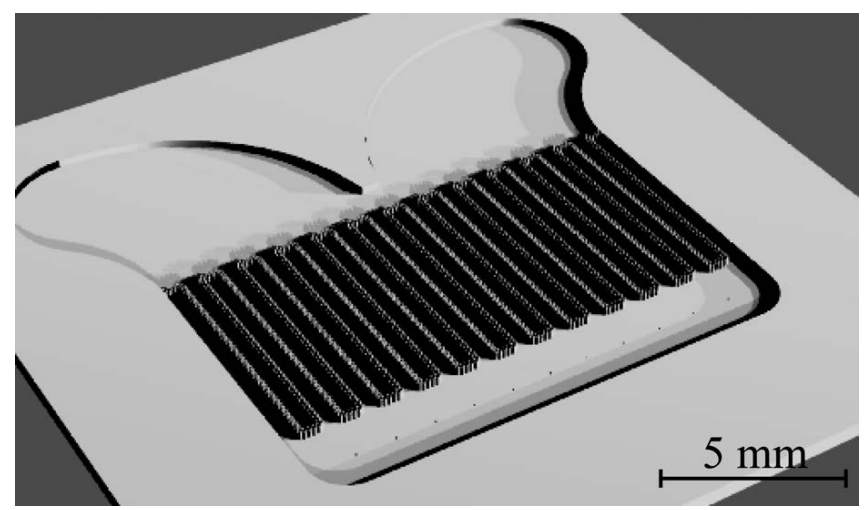

(a) Schematic representation of nozzle array.

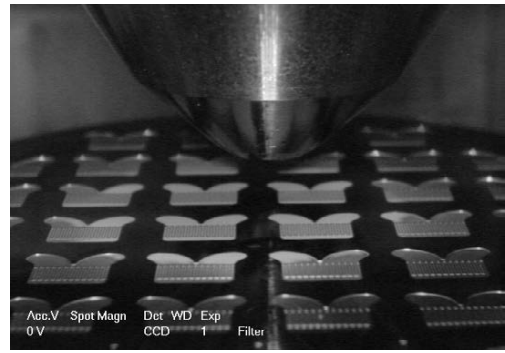

(b) Wafer before dicing.

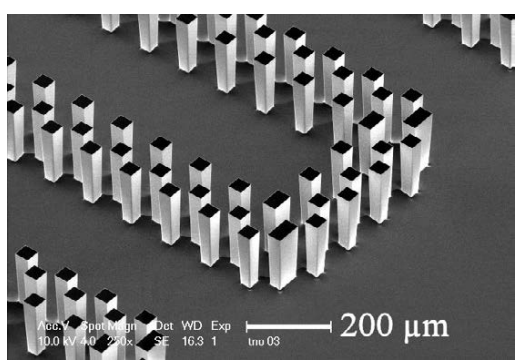

(c) Close-up of filtration structure.

Figure 7.2: Nozzle array created by microchip technology showing an integrated filtration structure.

\subsubsection{Integration of chemical processing within the printhead}

Nowadays it is common to make an ink, and feed this ink towards the deposition system used. Integration in the nozzle chip shown in figure 7.2 is a relatively simple material processing step, namely the filtration of the ink. Through the progress in micro-reactor technology, it has become possible to create much more complex fluidic structures on a chip. Taking a nozzle array as described as a starting point for integration with a micro-reactor-chip, offers whole new opportunities in our approach towards functional printing. Why spend millions on material development to improve the shelf life of your ink when it can be mixed inside the printhead from its base components when needed? 


\subsection{Concluding remarks}

Altogether for additive manufacturing to evolve towards a mature production technology, different machine layouts are required to bridge the gap from products a day towards products a minute or even seconds. The same can be said about inkjet technology. It has enormous possibilities, as long as the industry develops the technology needed to perform the specific task, instead of working on a technology route they have chosen in the past for whole different reasons. 


\section{Bibliography}

[1] J. E. Blanther, "Manufacture of contour relief maps", (1892), US 473901.

[2] C. Baese, "Photographic process for the reproduction of plastic objects", (1904), US 774549.

[3] C. W. Hull, "Apparatus for production of three-dimensional objects by stereolithography”, (1986), US 4575330.

[4] M. Feygin, "Apparatus and method for forming an integral object from laminations", (1988), US 4752352.

[5] C. R. Deckard, "Method and apparatus for producing parts by selective sintering”, (1989), US 4863538.

[6] L. E. Weiss, F. R. Prinz, and E. L. Gursoz, "Method and apparatus for fabrication of three-dimensional articles by thermal spray deposition", (1992), US 5126529 .

[7] W. E. Masters, "Computer automated manufacturing process and system", (1987), US 4665492.

[8] M. Yamane, T. Kawaguchi, S. Kagayama, S. Higashiyama, K. Suzuki, J. Sakai, M. Imaeda, and K. Inaishi, "Apparatus and method for forming threedimensional article", (1991), US 5059266.

[9] R. Helinski, "Method and means for constructing three-dimensional articles by particle deposition", (1992), US 5136515.

[10] T. A. Almquist and D. R. Smalley, "Thermal stereolighography”, (1992), US 5141680.

[11] E. M. Sachs, J. S. Haggerty, M. J. Cima, and P. A. Williams, "Threedimensional printing techniques", (1993), US 5204055. 
[12] H. P. Le, "Progress and trends in ink-jet printing technology", Journal of Imaging Science and Technology 42, 49-62 (1998).

[13] H. Wijshoff, "Structure- and fluid-dynamics in piezo inkjet printheads", Ph.D. thesis, University of Twente (2008).

[14] D. J. Hayes, M. E. Grove, and W. R. Cox, "Development and application by inkjet printing of advanced packaging materials", International symposium on adv packaging materials 88-93 (1999).

[15] S. F. Pond, Inkjet technology and product development strategies (Torrey Pines Research) (2000).

[16] E. R. Lee, Microdrop generation (CRC Press) (2003).

[17] R. J. Houben, "Apparatus and method for printing a fluid material by means of a continuous jet printing technique", (2004), WO 2004018212.

[18] R. J. Houben, "Constant flow high pressure printing system", (2008), WO 2008060149.

[19] R. J. Houben, L. A. M. Brouwers, and A. Rijfers, "Droplet break-up device", (2009), WO 2009028947.

[20] A. Rijfers, R. J. Houben, and L. A. M. Brouwers, "Droplet break-up device", (2009), WO 2009061202.

[21] G. P. H. Gubbels and R. J. Houben, "Droplet break-up device”, (2010), WO 2010068108.

[22] F. J. M. de Vreede, A. P. Aulbers, and R. J. Houben, "Pressure independent droplet generation", (2009), WO 2009151332.

[23] J. J. Michels and R. J. Houben, "Curable compositions for continuous inkjet printing and methods for using these compositions", (2007), EP 1869133.

[24] R. J. J. Boot, R. J. Houben, G. Oosterhuis, and A. P. Aulbers, "Droplet selection mechanism", (2009), WO 2009061201.

[25] R. J. J. Boot, R. J. Houben, G. Oosterhuis, and A. P. Aulbers, "Droplet selection mechanism", (2009), WO 2009061195.

[26] A. T. Poortinga and R. J. Houben, "Method and apparatus for spray drying and powder produced using said method", (2008), WO 2008069639. 
[27] R. J. Houben, A. Rijfers, L. A. M. Brouwers, J. Eversdijk, and K. J. C. van Bommel, "Multi component particle generation system", (2009), WO 2009014432.

[28] R. J. Houben, A. Rijfers, and L. A. M. Brouwers, "Multi component particle generating system", (2010), WO 2010090518.

[29] R. J. Houben, A. Rijfers, and L. A. M. Brouwers, "Multi component particle generating system", (2010), WO 2010005302.

[30] R. Cauchois, "Microstructuration of inkjet printed deposits: from silver nanoparticles coalescence to the fabrication of interconnections for electronic devices”, Ph.D. thesis, Ecole Nationale Supérieure des Mines (2012).

[31] R. J. Houben and G. Oosterhuis, "Method and apparatus for applying a material on a substrate", (2009), WO 2009011583.

[32] R. J. Houben, “Material jet system”, (2007), WO 2007075084.

[33] URL http://www.piezotechnologies.com/k12.htm.

[34] J. W. S. Rayleigh, "On the stability or instability of certain fluid motions", Rayleigh Scientific Papers 1 (1899).

[35] J. W. S. Rayleigh, "On the stability or instability of certain fluid motions 2", Rayleigh Scientific Papers 3 (1902).

[36] J. W. S. Rayleigh, "On the stability or instability of certain fluid motions 3", Rayleigh Scientific Papers 4 (1903).

[37] J. A. F. Plateau, "On the recent theories of the constitution of jets of liquid issuing from circular orifices”, Phil. Mag. 12, 286-297 (1856).

[38] C. Weber, "Zum Zerfall eines Flüssigkeitsstrahles", Z. für angew. Math. und mech. 11, 136-154 (1931).

[39] C. Clanet and J. C. Lasheras, "Transition from dripping to jetting", J. Fluid Mech. 383, 307-326 (1999).

[40] K. Matsushita, M. Sadakata, and T. Sakai, "Lower limit of vibration frequency, effect of viscosity and gravity on uniform droplet region by vibrating method", Proc. Int. Conf. Liquid Atomisation and Spray Systems 109-116 (1988). 
[41] R. Grant and S. Middleman, "Newtonian jet stability", A.I.Ch.E. Journal 12, 669-679 (1966).

[42] T. Sakai and N. Hoshino, "Production of uniform droplets by longitudinal vibration of audio frequency", Journal of Chemical Engineering of Japan 13, 263-268 (1980).

[43] P. Schummer and K. Tebel, "Production of monodispersed drops by forced longitudinal vibration of a liquid jet", Proc. Int. Conf. Liquid Atomisation and Spray Systems 47-54 (1982).

[44] T. Sakai, M. Sadakata, M. Saito, N. Hoshno, and S. Senuma, "Uniform size droplets by longitudinal vibration of newtonian and non-newtonian fluids", Proc. Int. Conf. Liquid Atomisation and Spray Systems 37-45 (1982).

[45] W. M. Beltman, "Viscothermal wave propagation including acousto-elastic interaction", Ph.D. thesis, University of Twente (1998).

[46] W. C. Young and R. G. Budynas, Roark's formulas for stress and strain, 7th edition (McGraw-Hill) (2002).

[47] URL http://www. imagexpert.com.

[48] URL http://www.hsps.com.

[49] J. A. van der Bos, "Air entrapment and drop formation in piezo inkjet printing", Ph.D. thesis, University of Twente (2011).

[50] S. Magdassi, The Chemistry of inkjet inks (World Scientific Publishing Co, Singapore) (2010).

[51] J. J. Sangiovanni and R. J. Michaud, "Ballistically controlled nonpolar droplet dispensing method and apparatus", (1982), US 4341310.

[52] G. H. Ko and H. S. Ryou, "Modelling of droplet collision-induced breakup process", International Journal of Multiphase Flow 31, 723-738 (2005).

[53] N. Ashgriz and J. Y. Poo, "Coalescence and separation in binary collisions of liquid drops", J. Fluid Mech. 221, 183-204 (1990).

[54] J. Estrade, H. Carentz, G. Lavergne, and Y. Biscos, "Experimental investigation of dynamic binary collision of ethanol droplets - a model for droplet coalescence and bouncing", International Journal of Heat and Fluid Flow 20, 486-491 (1999). 
[55] P. R. Brazier-Smith, S. Jennings, and J. Latham, "The interaction of falling water drops: coalescence", Proceedings of the Royal Society of London 326, 393-408 (1972).

[56] R. Chen, "Diesel-diesel and diesel-ethanol drop collisions", Applied Thermal Engineering 27, 604-610 (2007).

[57] J. H. Snoeijer, P. Brunet, and J. Eggers, "Maximum size of drops levitated by an air cushion", Physical review E 79, 036307 (2009).

[58] T. van Gils, "Ballistic droplet interception", Master's thesis, Technical university of Eindhoven (2008).

[59] R. B. Bird, W. E. Stewart, and E. N. Lightfoot, Transport Phenomena, 2nd edition (John Wiley \& Sons, Inc) (2002).

[60] G. Settles, Schlieren and shadowgraph techniques (Springer Verlag, Berlin) (2001).

[61] T. G. Theofanous, G. J. Li, and T. N. Dinh, "Aerobreakup in rarefied supersonic gas flows", Journal of Fluids Engineering 126, 516-527 (2004).

[62] B. E. Gelfand, "Droplet breakup phenomena in flows with velocity lag", Prog. Energy Combust. Sci. 22, 201265 (1996).

[63] K. Nomura, S. Koshizuka, Y. Oka, and H. Obata, "Numerical analysis of droplet breakup behaviour using particle method", Journal of Nuclear Science and Technology 38, 1057-1064 (2001).

[64] D. R. Guildenbecher, C. Lopez-Rivera, and P. R. Sojka, Handbook of Atomization and Sprays, chapter Droplet deformation and breakup, 145-156 (Springer) (2011).

[65] L. P. Hsiang and G. M. Faeth, "Near-limit drop deformation and secondary breakup", International Journal of Multiphase Flow 18, 635-652 (1992).

[66] E. Villermaux and B. Bossa, "Single-drop fragmentation determines size distribution of raindrops", Nature Physics 5, 697-702 (2009).

[67] S. A. Krzeczkowski, "Measurement of liquid droplet disintegration mechanisms", International Journal of Multiphase Flow 6, 227-239 (1980). 
[68] B. E. Gelfand, S. A. Gubin, S. M. Kogarko, and S. P. Komar, "Singularities of the breakup of viscous liquid droplets in shock waves", Journal of Engineering Physics and Thermophysics 25, 1140-1142 (1973).

[69] K. S. Rattan and V. Feliu, "Feedforward control of flexible manipulators", Int. Conf. on Robotics and Automation 788-793 (1992).

[70] P. F. J. Nouws, "Printing powders, inkjet-based droplet generation to improve powder properties and enable processing of highly viscous materials in spray dryers", Master's thesis, Stan Ackermans Institute, Eindhoven (2006).

[71] E. J. F. P. Ribeiro, "Printing powders scale-up", Master's thesis, Stan Ackermans Institute, Eindhoven (2007).

[72] A. Sarchami and N. Ashgriz, Handbook of Atomization and Sprays, chapter Splash Plate Atomizers, 709-726 (Springer) (2011).

[73] M. P. Fard, D. Levesque, S. Morrison, N. Ashgriz, and J. Mostaghimi, "Characterization of splash-plate atomizers using numerical simulations", Atomization and Sprays 17, 347-380 (2007).

[74] M. Nakayama and Y. Takahashi, "Experimental and theoretical investigation of longitudinal vibrations on liquid jets and sheets", Proc. Int. Conf. Liquid Atomisation and Spray Systems 1-8 (1982).

[75] N. Ashgriz and A. Sarchami, Handbook of Atomization and Sprays, chapter Instability of Liquid Sheets, 75-95 (Springer) (2011).

[76] L. T. Creagh and J. Caruso, "Industrial inkjet printing of single layer electronics", Speciality Graphic Imaging Association Membrane Switch Symposium (2007).

[77] URL http://www.optomec.com/.

[78] URL http://www. conductiveinkjet.com/.

[79] URL http://www.microfab.com/.

[80] K. C. Pingali, D. A. Rockstraw, and S. Deng, "Silver nanoparticles from ultrasonic spray pyrolysis of aqueous silver nitrate", Aerosol Science and Technology 39, 1010-1014 (2005).

[81] A. T. W. Kempen, "Method for applying material onto a substrate using a droplet printing technique", (2006), WO 2006004406. 
[82] T. Wohlers, Wohlers Report (Wohlers Associates) (2008).

[83] J. W. S. Rayleigh, "On the instability of jets", Proc. London Math. Soc. 10, 4-13 (1878).

[84] "Special report: Manufacturing and innovation: A third industrial revolution", The Economist (2012).

[85] URL http://www.tno.nl/rm.

[86] H. Koukka, "Rapid prototyping tree", Technical Report, Helsinki University of Technology (2004).

[87] E. V. Fudim, "Method and apparatus for production of three-dimensional objects by photosolidification", (1988), US 4752498.

[88] I. Pomerantz, J. Cohen-Sabban, A. Bieber, J. Kamir, M. Katz, and M. Nagler, “Three dimensional modelling apparatus", (1990), US 4961154.

[89] L. D. Mitcham and W. E. Nelson, "Stereolithographic apparatus and method of use", (1993), US 5247180.

[90] K. Silverbrook, "Method for creating a 3-d object", (2004), US 6997698.

[91] C. S. Taylor, W. B. Tiffany, P. Cherkas, J. J. Frantzen, H. J. Hampton, L. Nanis, and B. O. Shah, "Formation of three dimensional objects and assemblies", (1994), US 5348693.

[92] A. L. Cohen, "Method for electrochemical fabrication", (2000), US 6027630.

[93] W. Zhang, M. C. Leu, Z. Ji, and Y. Yan, "Method and apparatus for rapid freezing prototyping", (2001), US 6253116.

[94] S. L. Goodman and P. Campagnola, "Free-form fabrication using multi-photon excitation", (2001), US 6316153.

[95] R. Larsson, "Method and device for manufacturing three-dimensional bodies", (2003), US 6531086.

[96] G. K. Lewis, J. O. Milweski, D. A. Cremers, R. B. Nemec, and M. R. Barbe, "Laser production of articles from powders", (1998), US 5837960.

[97] F. P. Jeantette, D. M. Keicher, J. A. Romero, and L. P. Schanwald, "Method and system for producing complex-shape objects", (2000), US 6046426. 
[98] R. Larson, "Method and device for producing three-dimensional bodies", (1998), US 5786562.

[99] B. Khoshnevis, "Selective inhibition of bonding of power particles for layered fabrication of 3-d objects", (2003), US 6589471.

[100] T. Davidson, R. A. Phillips, A. T. Hernandes, D. B. Russell, K. Roche, W. H. Zengerle, A. Berlin, J. P. Kinsley, B. D. Sweet-Block, and D. Kisai, "Threedimensional printer", (2004), US 2004012112.

[101] R. Hoechsmann and I. Ederer, "Method for manufacturing of parts by a deposition technique", (2000), US 6147138.

[102] K. P. Hickerson, B. Bedal, and R. J. Diephuis, "Apparatus for three dimensional printing using image layers", (2005), US 7261542.

[103] S. S. Crump, "Apparatus and method for creating three-dimensional objects", (1992), US 5121329.

[104] B. Khoshevis, "Additive fabrication apparatus and method", (1996), US 5529471.

[105] D. P. Edmonds and M. D. McAninch, "Method and apparatus for building a workpiece by deposit welding", (1988), US 4775092.

[106] K. M. McHugh and J. F. Key, "Spray forming process for producing molds, dies and related tooling", (1998), US 5718863.

[107] W. N. Jenkins, “Spray deposition of metals”, (1995), US 5460851.

[108] D. W. Gore, "Method for producing a free-form solid-phase object from a material in the liquid phase", (1993), US 5257657.

[109] R. C. Sanders, J. L. Forsyth, and K. F. Philbrook, “3-d model maker”, (1996), US 5506607.

[110] N. F. Kinzie, "Method and apparatus for constructing a three-dimensional surface of predetermined shape and color", (1991), US 5015312.

[111] S. Morita and K. Sugiyama, "Sheet lamination modeling method and sheet lamination modeling apparatus", (2000), US 6056843.

[112] D. White, "Object consolidation employing friction joining", (2002), US 6457629. 
[113] G. V. Mathis, “Twin row drop generator", (1972), US 3701998.

[114] R. G. Sweet, "Fluid droplet recorder”, (1971), US 3596275.

[115] W. Ohnesorge, "Formation of drops by nozzles and the breakup of liquid jets", Journal of Applied Mathematics and Mechanics 16, 355-358 (1936).

[116] C. H. Hertz and S. Simonsson, "Ink jet recorder”, (1968), US 3416153.

[117] T. Yamada, "Ink-jet recording device with alternate small and large drops", (1978), US 4068241.

[118] C. R. Winston, "Method of and apparatus for transferring ink", (1962), US 3060429.

[119] E. Ascoli, "Verfahren und Vorrichtung zum Schreiben von Zeichen, Buchstaben od. dgl. mittels eines aus elektrisch geladenen Teilchen bestehenden Strahles", (1962), DE 1126422.

[120] URL http://www.genialab.com/TechJetCutter.php.

[121] R. T. Buck, F. L. Cloutier, R. E. Erni, R. N. Low, and F. D. Terry, "Disposable ink jet head", (1985), US 4500895.

[122] T. Hara, Y. Sato, Y. Takatori, and Y. Shirato, "Ink jet recording device using thermal propulsion and mechanical pressure changes", (1981), US 4296421.

[123] R. Brescia, M. Buat, G. Dagna, and A. Scardovi, "Selective ink-jet printing device", (1985), US 4502054.

[124] T. E. Johnson and K. W. Bower, "Review of the drop on-demand ink jet with primary emphasis on the gould jet concept", Journal of applied photographic engineering 5, 174-178 (1979).

[125] J. Heinzl, "Printing with ink droplets from a multi-nozzle device", Advances in non-impact printing technologies for computer and office applications, Joseph Gaynor Ed. 1191-1201 (1981).

[126] J. F. Dijksman, "Hydrodynamics of small tubular pumps", Journal of Fluid Mechanics 139, 173-191 (1984).

[127] W. S. Bartky, A. D. Paton, S. Temple, and A. J. Michaelis, "Droplet deposition apparatus", (1989), US 4879568. 
[128] A. J. Michaelis, A. D. Paton, S. Temple, and W. S. Bartky, "Droplet deposition apparatus", (1989), US 4887100.

[129] K. H. Fischbeck and A. T. Wright, "Shear mode transducer for drop-ondemand liquid ejector", (1986), US 4584590.

[130] B. T. Khuri-Yakub, S. A. Elrod, C. F. Quate, E. G. Rawson, and B. B. Hadimioglu, "Perforated membranes for liquid contronlin acoustic ink printing", (1991), US 5028937.

[131] C. F. Quate, E. G. Rawson, and B. B. Hadimioglu, "Multi-discrete-phase fresnel acoustic lenses and their application to acoustic ink printing", (1991), US 5041849 .

[132] N. Maehara, K. Hashido, and H. Hirata, "Piezoelectric oscillated nozzle", (1985), US 4533082.

[133] N. Maehara, "Ultrasonic liquid ejecting apparatus", (1986), US 4605167.

[134] H. Braun, "Synchronously stimulated continuous ink jet head", (2003), US 6505920.

[135] Y. Fujimura, K. Saito, E. Akutsu, N. Inoue, and K. Horie, "Thermal electrostatic ink-jet recording apparatus", (1988), US 4737803.

[136] K. Silverbrook, "Fax machine with concurrent drop selection and drop separation ink jet printing", (1998), US 5781202.

[137] S. Sohn, "Electrostatic hydrodynamic jet writing method using electrorheological fluid and apparatus thereof", (1996), US 5576747.

[138] P. Cielo and W. D. Westwood, "Printing by modulation of ink viscosity", (1979), US 4164745.

[139] R. Zengerle, N. Hey, H. Gruhler, M. Freygang, and M. Mueller, "Device and method for applying micro-droplets on a substrate", (2000), WO 0056443.

[140] H. Gruhler, N. Hey, H. Sandmaier, and R. Zengerle, "Method and device for applying a plurality of microdroplets onto a substrate", (2001), WO 0117669.

[141] R. Zengerle and B. de Heij, "Device and method for the non-contact application of micro-droplets on a substrate", (2004), EP 1405672. 
[142] G. Oosterhuis, F. Feenstra, R. Houben, B. van der Vorst, L. Brouwers, and F. Verhoeven, "Conductive metallic tracks by pyrolytic printing", Fourth Innovative Production Machines and Systems Virtual Conference 7-10 (2008).

[143] F. Feenstra, R. Houben, L. Brouwers, P. Hofmann, J. Fischer-Buehner, U. Klotz, G. Wagner, and P. Pierron, "Direct metal jetting", Fifth Innovative Production Machines and Systems Virtual Conference 1-4 (2009).

[144] R. J. Houben, L. A. M. Brouwers, A. Rijfers, and M. A. Willemse, "Inkjet printing for rapid manufacturing: Printing towards the future", Fifth Innovative Production Machines and Systems Virtual Conference 19-22 (2009). 


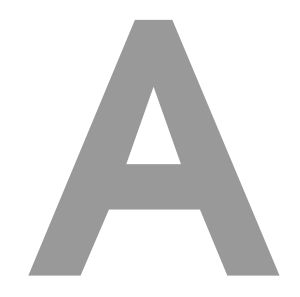

\section{Rapid manufacturing technology overview}

In this appendix an overview is given of layer-wise production techniques (based on an overview originally composed by [86]). Commercial available as well as processes only available in research environments are given. The list is non exhaustive, due to the speed of developments in the field. Its purpose is to show the broad spectrum of available techniques. The processes are divided in five categories; technologies based on liquids (table A.1), powders (table A.2), solid materials (table A.3), sheet materials (table A.4), and gasses (table A.5).

Table A.1: Rapid Manufacturing technology based on liquids.

\begin{tabular}{|c|c|c|}
\hline Selection one: & Selection two: & Technology: \\
\hline \multirow[t]{4}{*}{$\begin{array}{l}\text { Photocurable } \\
\text { liquids }\end{array}$} & \multirow[t]{4}{*}{$\begin{array}{l}\text { Curing by light } \\
\text { through masks }\end{array}$} & $\begin{array}{l}\text { Design Controlled Automated Fabrication } \\
\text { (DESCAF) by Light Sculpting Inc. [87] }\end{array}$ \\
\hline & & $\begin{array}{l}\text { Rapid Micro Product } \\
\text { (RMPD) by MicroTEC }\end{array}$ \\
\hline & & $\begin{array}{l}\text { Solid Ground Curing (SGC) by Cubital Inc. } \\
\text { [88] }\end{array}$ \\
\hline & & $\begin{array}{l}\text { Micro Stereolithography (MSLA) by Ecole } \\
\text { Polytechnique Federale de Lausanne } \\
\text { Continued on next page }\end{array}$ \\
\hline
\end{tabular}


Table A.1 - continued from previous page

\begin{tabular}{|c|c|c|}
\hline Selection one: & Selection two: & Technology: \\
\hline & \multirow[t]{2}{*}{$\begin{array}{ll}\text { Curing } & \text { with } \\
\text { a visible } & \text { light } \\
\text { laser } & \end{array}$} & $\begin{array}{l}\text { Computer Operated Laser Active Modeling } \\
\text { (COLAM) by Mitsui Ship Building Com- } \\
\text { pany }\end{array}$ \\
\hline & & Mark 1000 by 3D Systems \\
\hline & \multirow[t]{11}{*}{$\begin{array}{l}\text { Curing with a } \\
\text { uv-laser (single } \\
\text { beam) }\end{array}$} & $\begin{array}{l}\text { Laser Modell System (LMS) by Fockele \& } \\
\text { Schwarze }\end{array}$ \\
\hline & & Meiko by Meiko \\
\hline & & $\begin{array}{l}\text { Rapid Micro Product } \text { Development } \\
\text { (RMPD) by MicroTEC }\end{array}$ \\
\hline & & $\begin{array}{l}\text { Solid Creation System (SCS) by Sony or D- } \\
\text { MEC }\end{array}$ \\
\hline & & $\begin{array}{l}\text { Solid Laser Diode Plotter System (SLP) by } \\
\text { Denken }\end{array}$ \\
\hline & & $\begin{array}{l}\text { Stereolithography (SLA) by 3D Systems or } \\
\text { Aaroflex Inc. or Russian Academy of Sci- } \\
\text { ences [3] }\end{array}$ \\
\hline & & Soliform by Teijin-Seiki \\
\hline & & $\begin{array}{l}\text { Solid Object Ultra-Violet Laser Plotting } \\
\text { (SOUP) by Mitsubishi or CMET }\end{array}$ \\
\hline & & Stereos by 3D Systems \\
\hline & & UniRapid by Unirapid Inc. \\
\hline & & $\begin{array}{l}\text { Air Bubble Stereolithography (ABS) by } \\
\text { Osaka Sangyo University }\end{array}$ \\
\hline & \multirow[t]{3}{*}{$\begin{array}{l}\text { Curing with two } \\
\text { laser beams si- } \\
\text { multaneously }\end{array}$} & $\begin{array}{l}\text { Photochemical Machining (PCM) by } \\
\text { Formigraphics Inc. }\end{array}$ \\
\hline & & $\begin{array}{l}\text { Beam Interference Solidification (BIS) by } \\
\text { Batelle Development Corporation }\end{array}$ \\
\hline & & $\begin{array}{l}\text { Holographic Interference Solidification } \\
\text { (HIS) by Quadatec }\end{array}$ \\
\hline & $\begin{array}{l}\text { Curing by vis- } \\
\text { ible light with } \\
\text { a DMD (Digi- } \\
\text { tal Micromirror } \\
\text { Device) [89] }\end{array}$ & $\begin{array}{l}\text { Direct Photo Shaping (DPS) by SRI Inter- } \\
\text { national }\end{array}$ \\
\hline & & Continued on next page \\
\hline
\end{tabular}


Table A.1 - continued from previous page

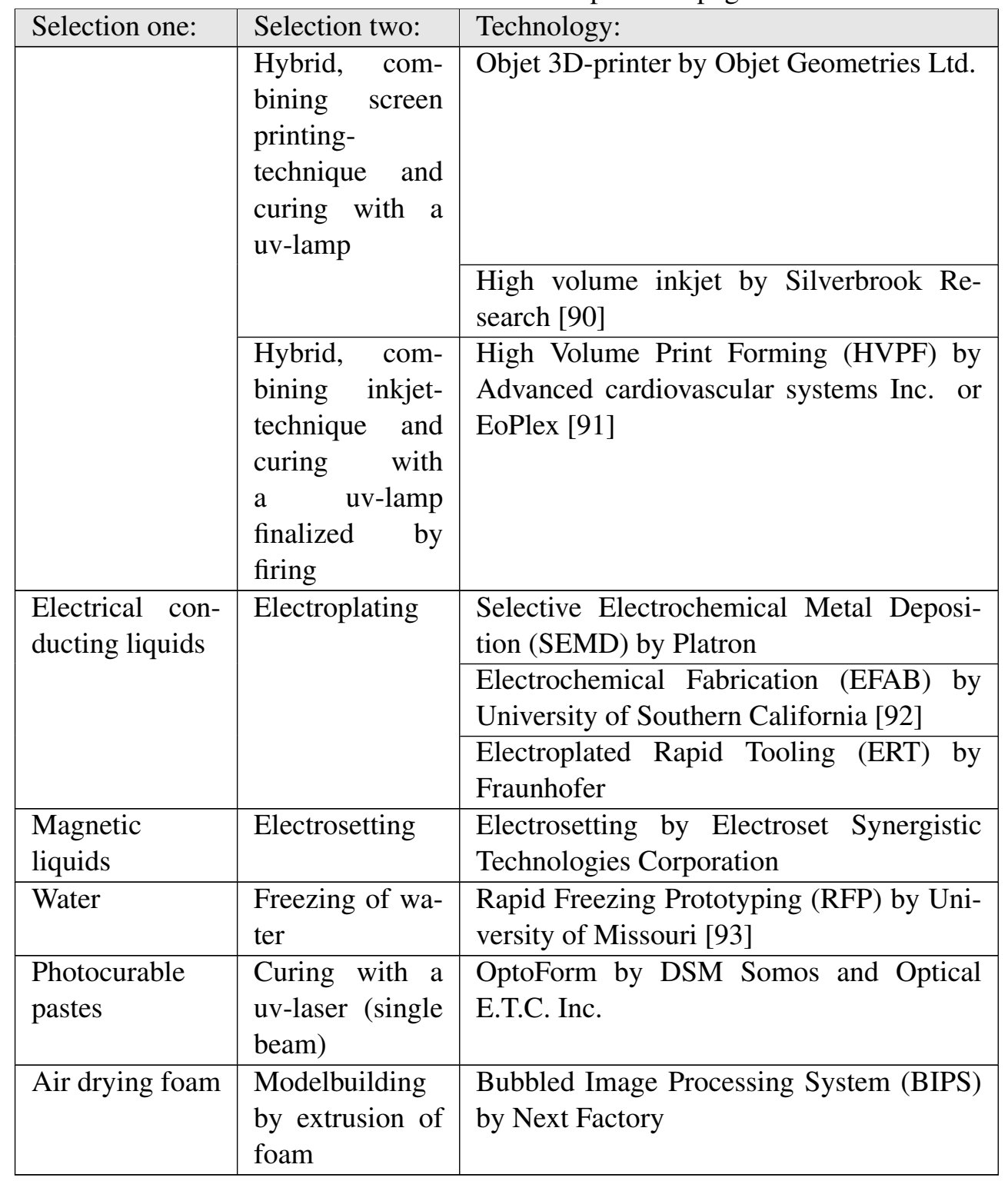


Table A.2: Rapid Manufacturing technology based on powders.

\begin{tabular}{|c|c|c|}
\hline Selection one: & Selection two: & Technology: \\
\hline \multirow[t]{16}{*}{$\begin{array}{l}\text { Melting of pow- } \\
\text { der }\end{array}$} & \multirow{6}{*}{$\begin{array}{l}\text { Sintering with } \\
\text { a heat transfer- } \\
\text { ring laser }\end{array}$} & $\begin{array}{l}\text { Selective Laser Sintering (SLS) by DTM } \\
\text { Corporation [5] }\end{array}$ \\
\hline & & $\begin{array}{l}\text { Compacted-powder SLS by University of } \\
\text { Manchester }\end{array}$ \\
\hline & & $\begin{array}{l}\text { Direct Metal Fabrication (DMF) by Rock- } \\
\text { well Scientific }\end{array}$ \\
\hline & & $\begin{array}{l}\text { Selective Laser Reaction Sintering (SLRS) } \\
\text { by University of Conneticut [94] }\end{array}$ \\
\hline & & $\begin{array}{l}\text { Selective Laser Sintering of ceramics (SLS- } \\
\text { ceramics) by Rheinisch-Westflische Tech- } \\
\text { nische Hochschule Aachen }\end{array}$ \\
\hline & & $\begin{array}{l}\text { Selective Laser Sintering variant by Speed } \\
\text { Part [95] }\end{array}$ \\
\hline & \multirow[t]{10}{*}{$\begin{array}{l}\text { Melting with a } \\
\text { heat transfer- } \\
\text { ring laser }\end{array}$} & $\begin{array}{l}\text { Direct Metal Laser Sintering (DMLS) by } \\
\text { EOS }\end{array}$ \\
\hline & & $\begin{array}{l}\text { Controlled Metal Buildup (CMB) by Fraun- } \\
\text { hofer }\end{array}$ \\
\hline & & Direct Laser Sintering (DLS) by Electrolux \\
\hline & & $\begin{array}{l}\text { Direct Metal Deposition (DMD) by Pre- } \\
\text { cision Optical Manufacturing/University of } \\
\text { Michigan }\end{array}$ \\
\hline & & $\begin{array}{l}\text { Laser Additive Manufacturing (LAM) by } \\
\text { AeroMet }\end{array}$ \\
\hline & & LaserCUSING by Hofmann \\
\hline & & Lasform by AeroMet \\
\hline & & $\begin{array}{l}\text { Laser Engineered Net Schaping (LENS) } \\
\text { (also known as: Direct Material Deposition } \\
\text { System (DMDS)) by Optomec/Sandia Na- } \\
\text { tional Laboratories }[96,97]\end{array}$ \\
\hline & & $\begin{array}{l}\text { Selective Laser Melting (SLM) by Fraun- } \\
\text { hofer }\end{array}$ \\
\hline & & Continued on next page \\
\hline
\end{tabular}


Table A. 2 - continued from previous page

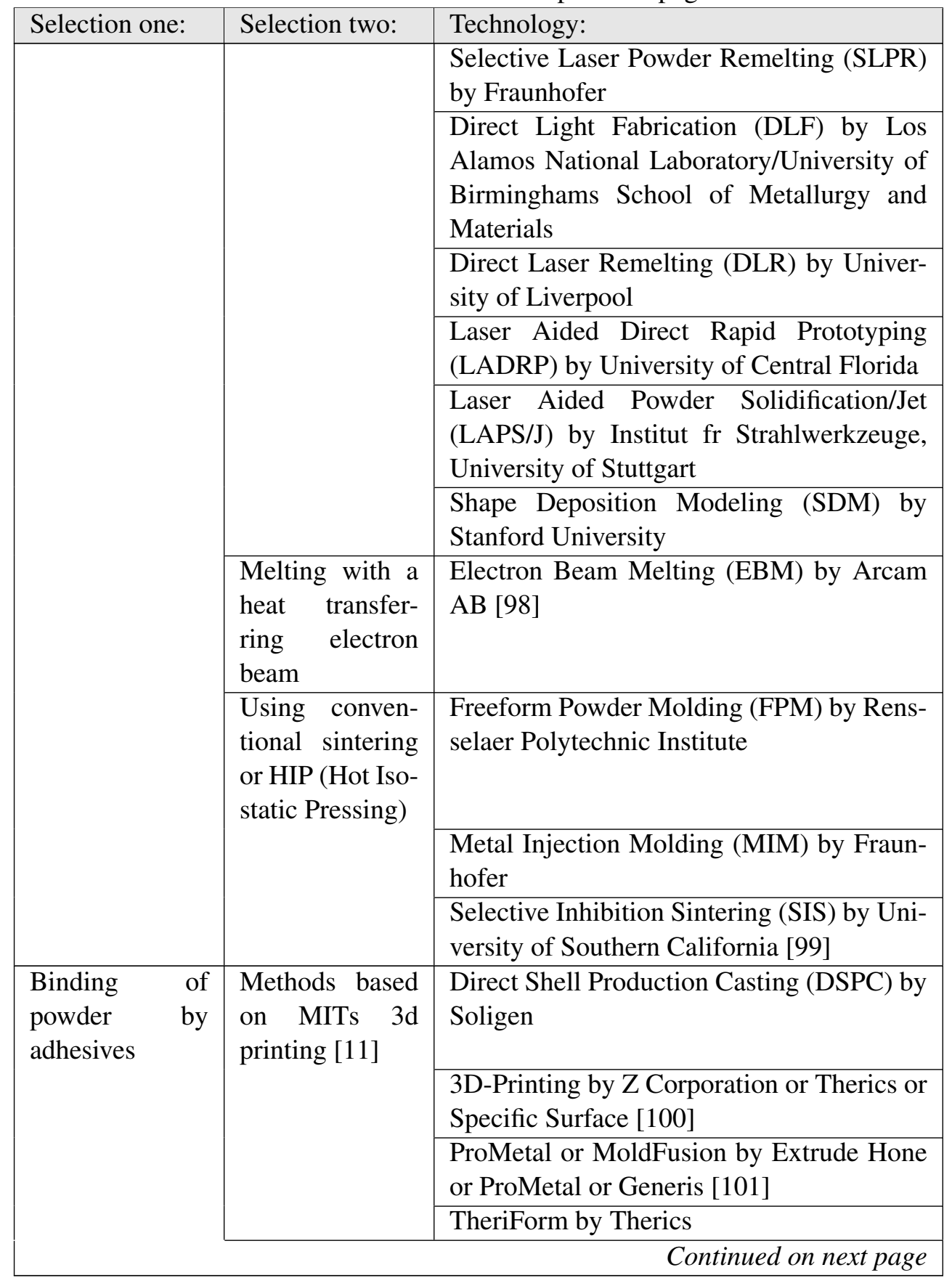


Table A. 2 - continued from previous page

\begin{tabular}{|l|l|l|}
\hline Selection one: & Selection two: & Technology: \\
\hline & $\begin{array}{l}\text { Extrusion of } \\
\text { ceramics with } \\
\text { melted binder }\end{array}$ & $\begin{array}{l}\text { Fused Deposition of Ceramics (FDC) by } \\
\text { Rutgers University }\end{array}$ \\
\hline Other methods & $\begin{array}{l}\text { Multiphase Jet Solidification (MJS) by } \\
\text { Fraunhofer IFAM and Fraunhofer IPA }\end{array}$ \\
& $\begin{array}{l}\text { Topographic Shell Fabrication (TSF) by } \\
\text { Formus }\end{array}$ \\
& $\begin{array}{l}\text { Powder Transfer by Desktop Factory Inc. } \\
{[102]}\end{array}$ \\
\hline
\end{tabular}

Table A.3: Rapid Manufacturing technology based on solid materials.

\begin{tabular}{|c|c|c|}
\hline Selection one: & Selection two: & Technology: \\
\hline \multirow[t]{13}{*}{$\begin{array}{l}\text { Extrusion of } \\
\text { melted material }\end{array}$} & \multirow[t]{3}{*}{$\begin{array}{l}\text { Extrusion of } \\
\text { plastics }\end{array}$} & $\begin{array}{l}\text { Fused Deposition Modelling (FDM) by } \\
\text { Stratasys [103] }\end{array}$ \\
\hline & & $\begin{array}{l}\text { Melted Extrusion Manufacturing (MEM) } \\
\text { by CLRF, Tsinghua University }\end{array}$ \\
\hline & & $\begin{array}{l}\text { Contour Crafting (CC) by University of } \\
\text { Southern California [104] }\end{array}$ \\
\hline & \multirow[t]{6}{*}{$\begin{array}{l}\text { Methods based } \\
\text { on welding }\end{array}$} & $\begin{array}{l}\text { Droplet Welding (DROW) by Aerochem } \\
\text { Research Laboratory, Division of Titan } \\
\text { Corporation }\end{array}$ \\
\hline & & 3D-Welding by Cranfield University \\
\hline & & $\begin{array}{l}\text { Extrusion and Deposition of Semi Solid } \\
\text { Metals (EDSSM) by TNO }\end{array}$ \\
\hline & & $\begin{array}{l}\text { Robot Fusion Welding (RFW) by Universi- } \\
\text { dade de Minho in Guimares, Portugal }\end{array}$ \\
\hline & & $\begin{array}{l}\text { Shape Melting (SM) by Babcock \& Wilcox } \\
\text { [105] }\end{array}$ \\
\hline & & Shape Welding (SW) by Thyssen Ag. \\
\hline & \multirow[t]{4}{*}{$\begin{array}{l}\text { Spraying of } \\
\text { metal }\end{array}$} & $\begin{array}{l}\text { Recursive Mask and Deposit (MD) by } \\
\text { Carnegie Mellon University [6] }\end{array}$ \\
\hline & & $\begin{array}{l}\text { Incremental Fabrication Technology (IFT) } \\
\text { by Incre LLC }\end{array}$ \\
\hline & & $\begin{array}{l}\text { Rapid Solidification Process (RSP) by RSP } \\
\text { Tooling LLC }[106,107]\end{array}$ \\
\hline & & Continued on next page \\
\hline
\end{tabular}


Table A. 3 - continued from previous page

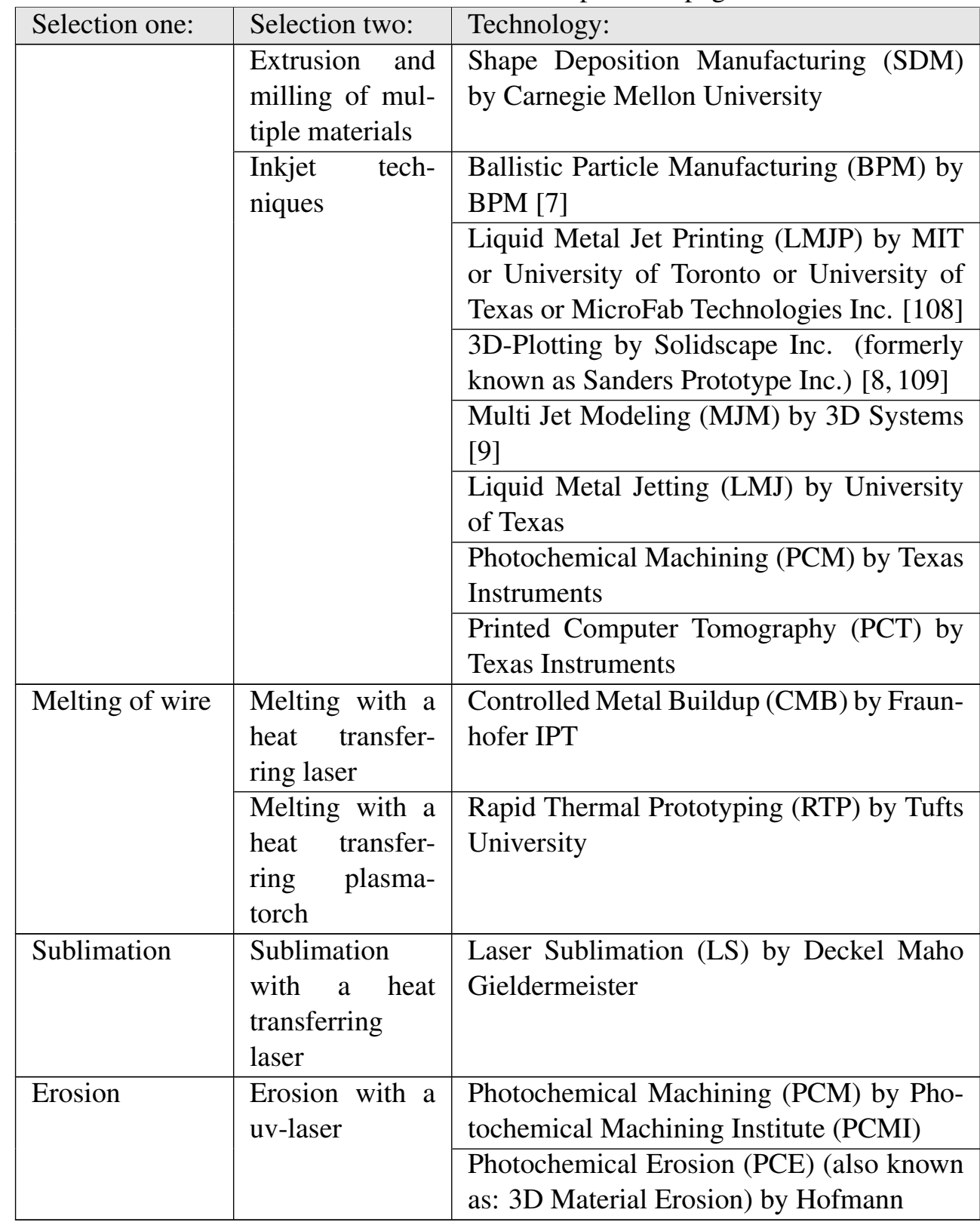


Table A.4: Rapid Manufacturing technology based on sheet materials.

\begin{tabular}{|c|c|c|}
\hline Selection one: & Selection two: & Technology: \\
\hline \multirow[t]{8}{*}{$\begin{array}{l}\text { Bond-first lami- } \\
\text { nation }\end{array}$} & \multirow[t]{5}{*}{$\begin{array}{l}\text { Cutting material } \\
\text { with a laser }\end{array}$} & $\begin{array}{l}\text { Computer-Aided Manufacturing of Lami- } \\
\text { nated Engineering Materials (CAM-LEM) } \\
\text { by CAM-LEM Inc. or Case Western Re- } \\
\text { serve University }\end{array}$ \\
\hline & & $\begin{array}{l}\text { Laminated Object Manufacturing (LOM) } \\
\text { by Helisys Inc. or Landfoam Topographics } \\
{[110]}\end{array}$ \\
\hline & & $\begin{array}{l}\text { Slicing Solid Manufacturing (SSM) by } \\
\text { CLRF, Tsinghua University }\end{array}$ \\
\hline & & $\begin{array}{l}\text { Curved-layer LOM by University of Day- } \\
\text { ton/Helisys Inc. }\end{array}$ \\
\hline & & LOM-ceramic by Ceramic Composite Inc. \\
\hline & \multirow[t]{2}{*}{$\begin{array}{l}\text { Cutting material } \\
\text { with a knife }\end{array}$} & $\begin{array}{l}\text { Paper Lamination Technology (PLT) by } \\
\text { Kira Corporation }[110,111]\end{array}$ \\
\hline & & $\begin{array}{l}\text { Selective Adhesive Hot Print (SAHP) by } \\
\text { Kira Corporation }\end{array}$ \\
\hline & $\begin{array}{l}\text { Cutting material } \\
\text { with a milling } \\
\text { machine }\end{array}$ & $\begin{array}{l}\text { Layer Milling (LM) by Helsinky University } \\
\text { of Technology or Pro Tooling }\end{array}$ \\
\hline \multirow[t]{8}{*}{$\begin{array}{l}\text { Cut-first lami- } \\
\text { nation }\end{array}$} & $\begin{array}{l}\text { Cutting material } \\
\text { with a laser }\end{array}$ & $\begin{array}{l}\text { Laser Profiling Machine (LPM) by Liptool } \\
\text { Ltd. }\end{array}$ \\
\hline & \multirow[t]{3}{*}{$\begin{array}{l}\text { Cutting material } \\
\text { with a knife }\end{array}$} & $\begin{array}{l}\text { JP System } 5 \text { by Schroff Development Cor- } \\
\text { poration }\end{array}$ \\
\hline & & Offset Fabrication by Ennex Corporation \\
\hline & & $\begin{array}{l}\text { Plastic Lamination Machine (PLM) by So- } \\
\text { lidimension [110] }\end{array}$ \\
\hline & \multirow[t]{2}{*}{$\begin{array}{l}\text { Cutting material } \\
\text { with a milling } \\
\text { machine }\end{array}$} & Solidica by Solidica Inc. [112] \\
\hline & & Strato Conception by Charlyrobot or Cirtes \\
\hline & $\begin{array}{l}\text { Cutting material } \\
\text { by water }\end{array}$ & TruSurf by Gilmore Engineers Pty Ltd. \\
\hline & & Continued on next page \\
\hline
\end{tabular}


Table A.4 - continued from previous page

\begin{tabular}{|l|l|l|}
\hline Selection one: & Selection two: & Technology: \\
\hline & $\begin{array}{l}\text { Cutting material } \\
\text { with a heated } \\
\text { electrode }\end{array}$ & HotPlot by Sparx AB \\
\cline { 3 - 3 } & & ShapeMaker II by University of Utah \\
\hline
\end{tabular}

Table A.5: Rapid Manufacturing technology based on gasses.

\begin{tabular}{|c|}
\hline \\
\hline Gas Phase Deposition (GPD) by University of Texas \\
\hline $\begin{array}{l}\text { Laser-Assisted Selective Area Metal Organic Chemical Vapor Deposition } \\
\text { (LASAMOCVD) }\end{array}$ \\
\hline Laser-Assisted Chemical Vapor Deposition (LCVD) by Georgia Institute \\
\hline Selective Area Laser Deposition (SALD) by University of Conneticut \\
\hline $\begin{array}{l}\text { Selective Area Laser Deposition Vapor Infiltration (SALDVI) by University of } \\
\text { Conneticut }\end{array}$ \\
\hline $\begin{array}{l}\text { Selective Laser Reaction Sintering (SLRS) by University of Conneticut or Univer } \\
\text { sity of Texas }\end{array}$ \\
\hline
\end{tabular}




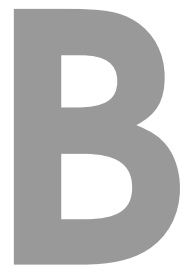

\section{Overview of available droplet formation methods}

In this appendix an overview is given of available droplet formation methods, subdivided in principles applicable for continuous inkjet or drop-on-demand systems. The images used are taken from patent literature of the technique described, and a detailed description of the reference numbers can be found in the cited patent.

\section{B.1 Continuous inkjet principles}

Several continuous inkjet principles can be distinguished. The following are described in more detail in this paragraph:

$\diamond$ Acoustically disrupted fluid jet

$\diamond$ Hertz mist

$\diamond$ MicroDot

$\diamond$ Electrohydrodynamic inkjet/ Electrostatic pull inkjet

$\diamond$ Jet cutter technology

\section{B.1.1 Acoustically disrupted fluid jet}

This is in fact the basic continuous inkjet principle, where the ink is pressurized out of an outflow opening, and so creating a jet. Depending on the type of disrupted fluid 
jet either the natural break-up effect is used or the break-up is actively controlled. Actively controlling the break-up can be achieved by introducing the correct perturbation in the fluid jet resulting in stable drop break-up. Piezoelectric elements are commonly used to introduce this vibration into the fluid, although thermal actuation is also used. Depending on the type of droplet selection and whether or not controlled drop formation is used, the drop selection can be subdivided into:

Binary deflection systems Drop selection is realized by selectively charging the droplets and jetting them through an electrical field. Charged droplets are pulled out of the main stream and deflected towards a recycling gutter; uncharged droplets are directed towards the substrate. Or the other way around: uncharged droplets recycled; charged droplets towards substrate (figure B.1).

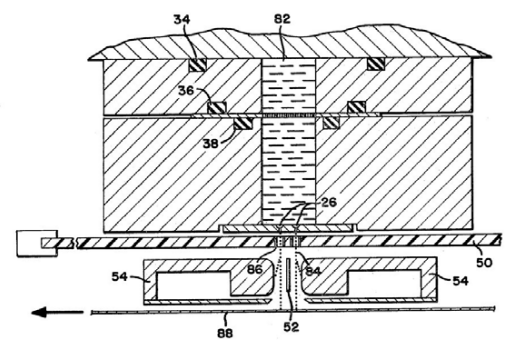

Figure B.1: Binary deflection system; The Mead Corporation, 1972 [113].

Multiple deflection systems Drop selection is realized by selectively charging the droplets, however, each droplet can be charged differently. In this case, the charged droplets are directed towards the substrate and uncharged droplets are jetted into the recycling gutter. Dependent on the charge level, a specific deflection is realized. This allows printing over a certain height on the substrate using a single nozzle (figure B.2).

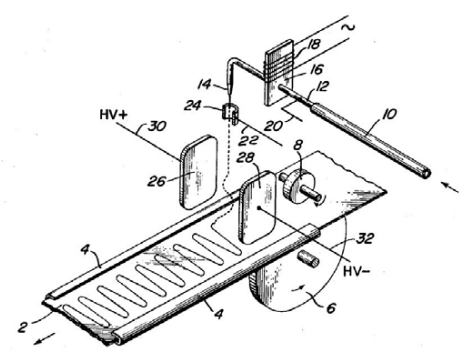

Figure B.2: Multi deflection system; Honeywell Regulator Co, 1971 [114]. 


\section{B.1.2 Hertz mist}

A very small nozzle is used with a relative high speed jet. In contrary to the above mentioned methods, natural break up of the jet is used [115]. Due to the small nozzle size and high ejection speed the resulting droplet generation frequency is higher, generating a stream of very small droplets. With the Hertz principle the droplets are not charged individually. If no charge is applied at all, the droplets will fly straight to the substrate resulting in a dense, sharp dot/line. If the droplets are slightly charged they will repel each other resulting in a vaguer image. However due to the small size of the droplets, this is imaged as a grayscale line. By varying the charge the focus of the deposited image can be adjusted enabling grayscales to be printed (figure B.3).

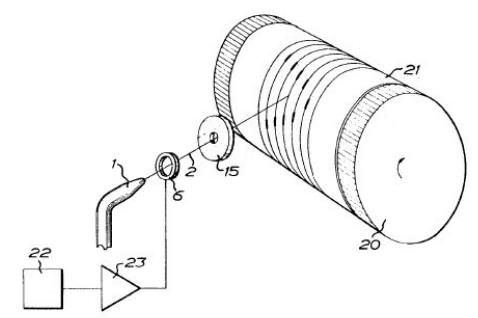

Figure B.3: Hertz technology; Hertz, 1968 [116].

\section{B.1.3 MicroDot}

MircoDot systems use controlled droplet break-up. Normally satellite droplets are undesirable with continuous inkjet systems, but with MicroDot, these satellites are actively used. The large drops are collected in the return gutter and the satellite droplets are selectively deposited on the substrate, enabling higher resolutions (figure B.4).

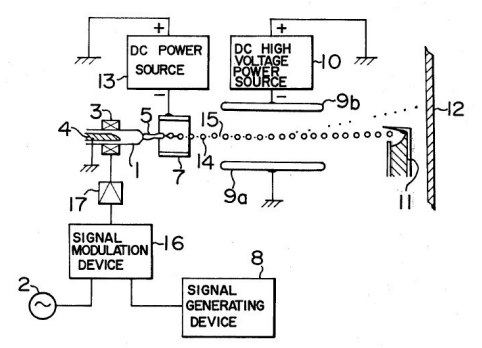

Figure B.4: MicroDot system; Hitachi LTD, 1978 [117]. 


\section{B.1.4 Electrohydrodynamic inkjet/ Electrostatic pull inkjet}

This principle uses conductive ink which is held in a nozzle under negative pressure. A droplet is ejected by applying a high voltage pulse to an electrode located outside the nozzle. This charged droplet can subsequently be directed to a specific location on the substrate by means of a deflection field. This principle was actually the first commercial success based on a drop-on-demand principle. The Casio Computer Company manufactured approximately 8000 units in 1970s, years before HP released it renowned thermal based Thinkjet ${ }^{t m}$. Casio licensed the process from Teletype (figure B.5) and Paillard company (figure B.6), which made their first products in the 1960s.

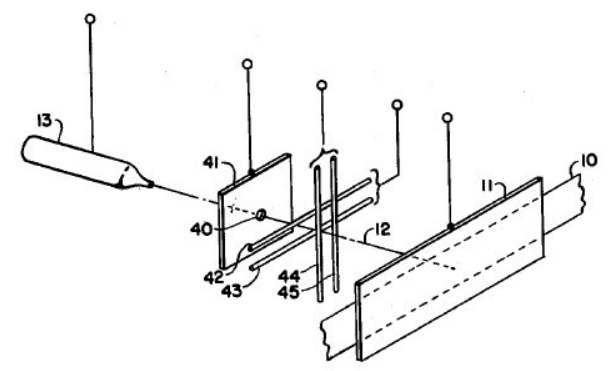

Figure B.5: Electrostatic pull inkjet; Teletype Corporation, 1962 [118].

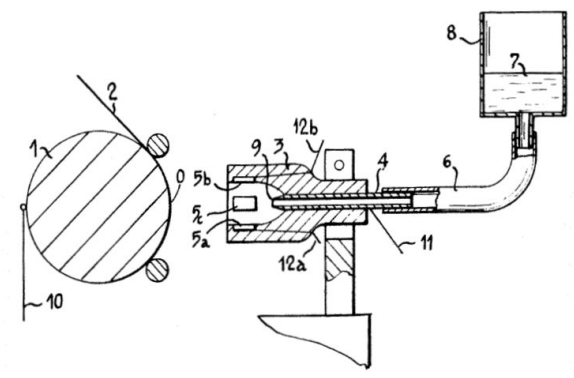

Figure B.6: Electrostatic pull inkjet; Paillard S.A., 1962 [119]. 


\section{B.1.5 Jet cutter technology}

Jet cutter technology doesn't rely on Rayleigh break-up for drop formation. With this method a liquid jet is ejected from a nozzle. Before the jet naturally breaks up into droplets the jet is mechanically cut into pieces, overruling natural break up (figure B.7). The process isn't commonly used for inkjet applications, rather for bead formation. In contrary to conventional inkjet principles, this process is capable of creating droplets with very high viscosity materials.

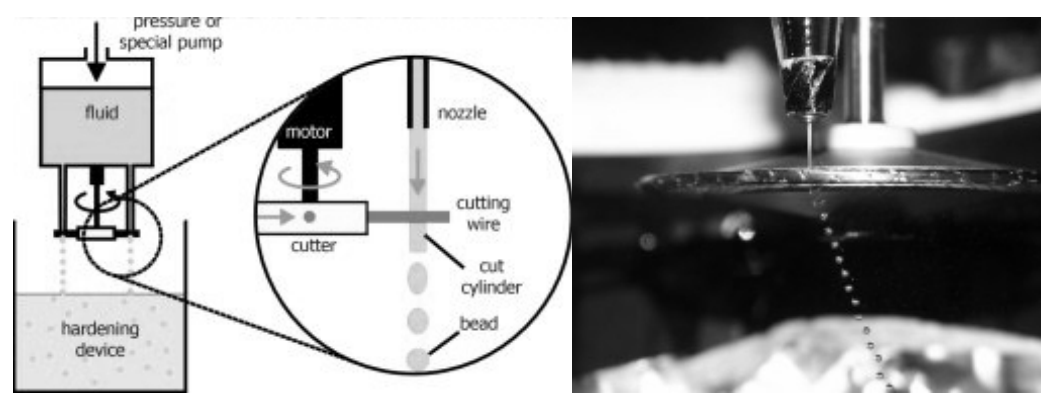

Figure B.7: Jet Cutter technology [120].

\section{B.2 Drop-on-demand principles}

Several drop-on-demand principles can be distinguished. The following are described in more detail in this paragraph:

$\diamond$ Thermal inkjet

$\diamond$ Thermal spark

$\diamond$ Piezoelectric direct pressure pulse

$\diamond$ Focused acoustic beam ejection

$\diamond$ Flex tensional aperture plate inkjet

$\diamond$ Thermal electrostatic inkjet

$\diamond$ Liquid fault tolerant process

$\diamond$ Electro-rheological fluid inkjet

$\diamond$ Thermal-rheological fluid inkjet

$\diamond$ Topspot microdrop ejector 


\section{B.2.1 Thermal inkjet}

Thermal inkjet uses a resistor which is pulsed to boil a small amount of ink at high temperatures (of over $300^{\circ} \mathrm{C}$ ). The fast expansion causes a shockwave in the channel causing a large pressure pulse, driving droplet ejection (figure B.8). The boiling action limits the number of usable inks, as well as the lifetime of such a device. Every ejection and boiling might lead to adhesion or debris formation in the printhead. The design however is suited for miniaturization allowing the use of MEMS technology. Cheap integration of the printhead chip with the ink reservoir enabled the creation of disposable printheads [121].

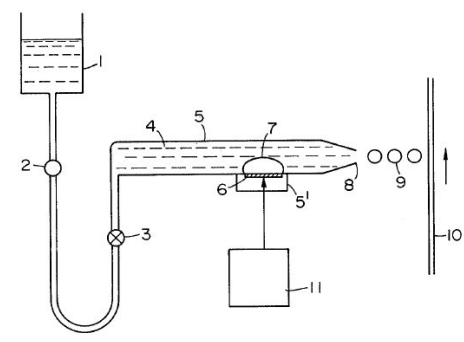

Figure B.8: Thermal inkjet; Canon, 1981 [122].

\section{B.2.2 Thermal spark}

Thermal spark inkjet is similar to thermal inkjet, however no resistor is used to evaporate the ink. An electrical discharge causes the conductive ink to vaporize. The expanding gas propels the ink towards the substrate. The initial pulse causes a fine spray of droplets to reach the substrate. Depending on the printhead design, a secondary droplet is propelled due to refilling of the nozzle (figure B.9).
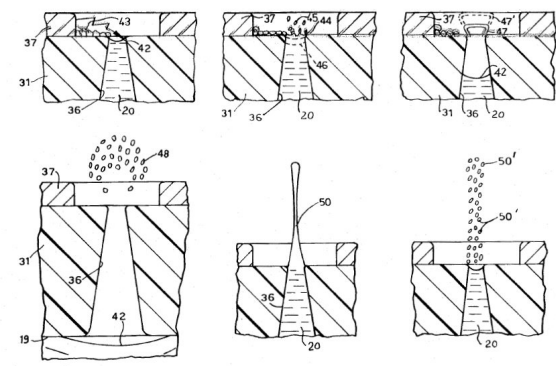

Figure B.9: Liquid thermal spark ejection; Olivetti, 1985 [123]. 


\section{B.2.3 Piezoelectric direct pressure pulse}

One of the most commonly used actuation mechanisms in industrial printing applications is piezo actuation. In these systems a piezo element is used to introduce a shockwave into the system. Depending on the layout of the printhead, several types can be distinguished.

Squeeze-mode: Goulds impulse inkjet, Siemens PT-80, MicroFab, MicroDrop [124126] (see figure B.10a).

Bend-mode: Tektronix Phaser, Epson Stylus (see figure B.10b).

Push-mode: Dataproducts, Trident (see figure B.10c).

Shear-mode: Xaar [127, 128], Dimatix Spectra [129] (see figure B.10d).

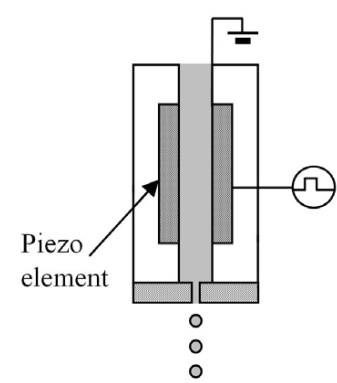

(a) Squeeze-mode

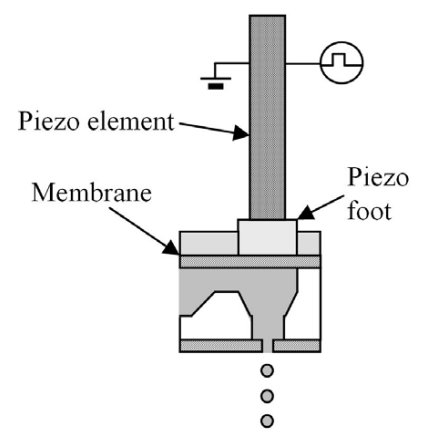

(c) Push-mode

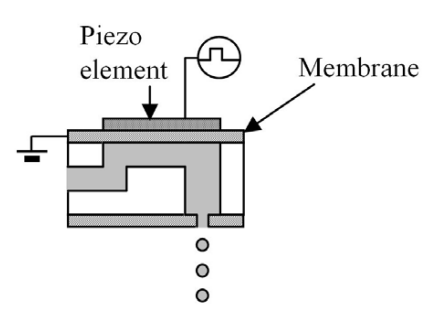

(b) Bend-mode

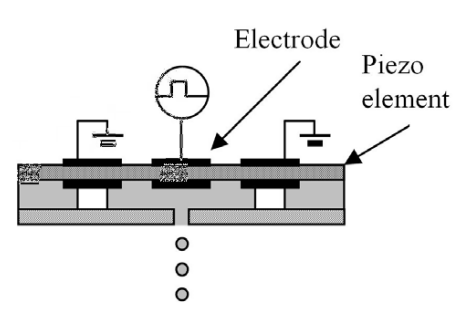

(d) Shear-mode

Figure B.10: Piezoelectric direct pressure pulse layouts. 


\section{B.2.4 Focused acoustic beam ejection}

Focused acoustic beam ejection emphases on the focusing of (piezo) energy to eject a droplet. The energy is focused on the surface by means of an acoustic lens, resulting in the ejection of a droplet from the surface if the energy intensity is high enough (figure B.11). To reduce the building height of such a structure and to simplify its manufacture, Fresnel lenses can be used as well (figure B.12).

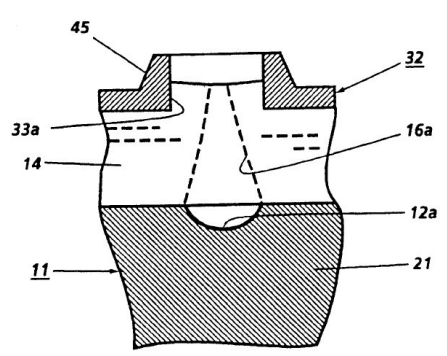

Figure B.11: Focused acoustic beam ejection; Xerox Corporation, 1991 [130].

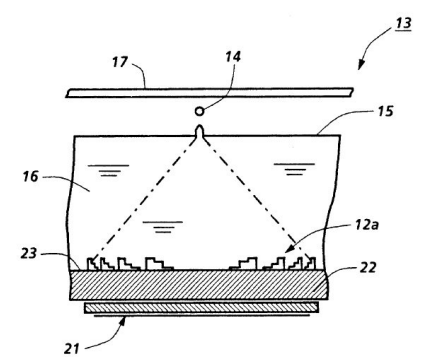

Figure B.12: Focused acoustic beam ejection with Fresnel lens; Xerox Corporation, 1991 [131].

\section{B.2.5 Flex tensional aperture plate inkjet}

Initially flex tensional aperture plate inkjet refers to designs that integrate the actuation mechanism in the nozzle plate, using the flexibility of the nozzle plate itself (figures B.13, B.14 and B.15). However the term is more commonly used for all principles using a vibrating nozzle design, which does not necessarily mean that the nozzle is actually flexible (figure B.16). 


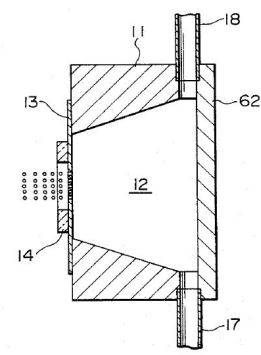

Figure B.13: Flex tensional aperture plate inkjet; Matsushita Electric Industrial Company, 1985 [132].

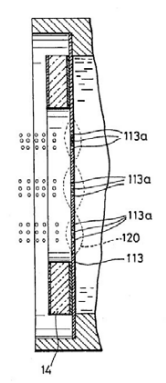

Figure B.14: Flex tensional aperture plate inkjet; Matsushita Electric Industrial Company, 1986 [133].

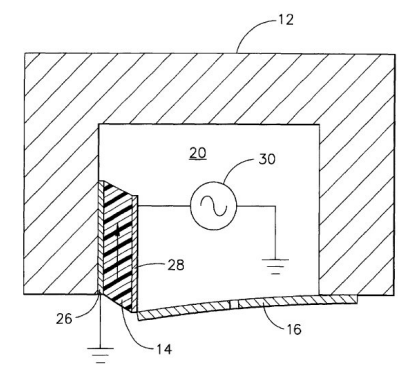

Figure B.15: Flex tensional aperture plate inkjet; Scitex Digital Printing Inc., 2003 [134]. 


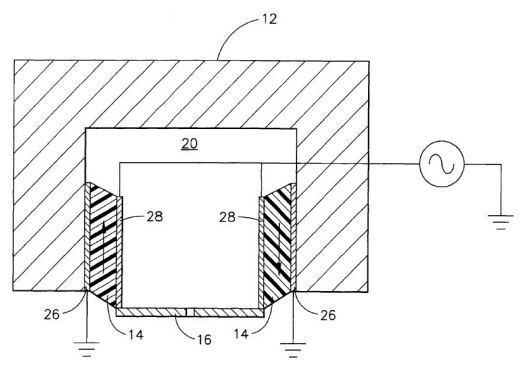

Figure B.16: Flex tensional aperture plate inkjet; Scitex Digital Printing Inc., 2003 [134].

\section{B.2.6 Thermal electrostatic inkjet}

Thermal electrostatic inkjet is similar to electrostatic pull inkjet, however thermal energy is used to selectively lower the viscosity of the ink inside the selected nozzle, enabling fluid ejection from this specific nozzle. This way the electrostatic pull principle was made applicable for drop-on-demand applications (figure B.17).
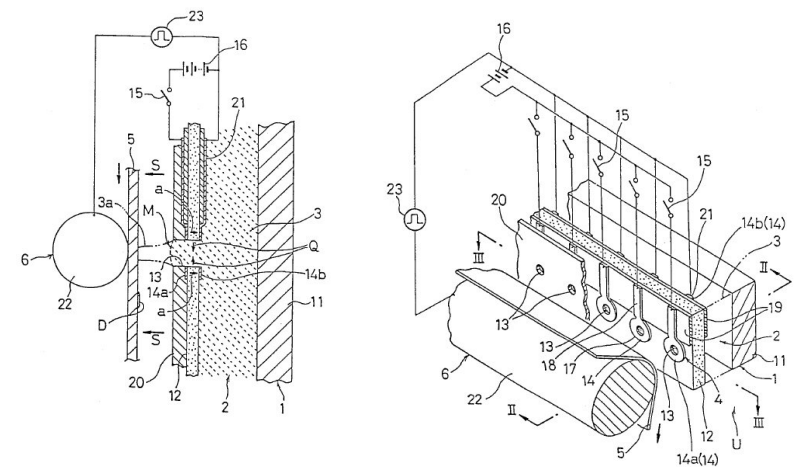

Figure B.17: Thermal electrostatic inkjet; Fuji Xerox, 1988 [135].

\section{B.2.7 Liquid fault tolerant process}

Liquid fault tolerant printing, also known as the LIFT process, is also a variation of electrostatic pull inkjet. With the LIFT process an electrostatic field is applied constantly, trying to pull the ink out of the nozzle. The surface tension prevents this. The ink is designed to lower its surface tension upon heating, enabling drop ejection 
if the ink is subjected to heat pulses (figure B.18). It can be debated whether there is a large difference between thermal electrostatic pull and the LIFT process, since both viscosity and surface tension are temperature dependent. The technology is aimed at generating a cost effective page-wide marking process.

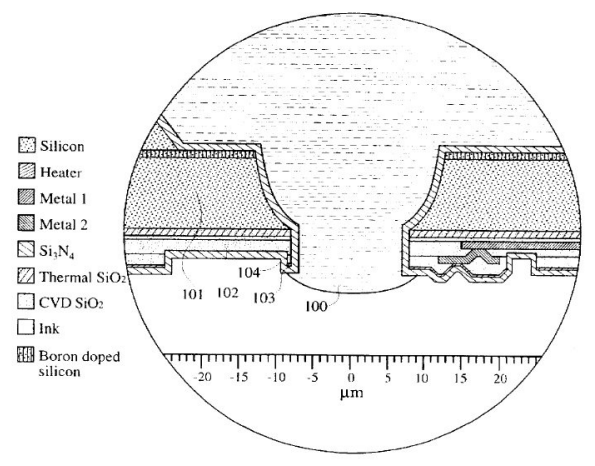

Figure B.18: LIFT; Eastman Kodak Company, 1998 [136].

\section{B.2.8 Electro-rheological fluid inkjet}

Similar to the LIFT and Thermal electrostatic inkjet, Electro-rheological fluid inkjet relies on changing fluid parameters to selectively eject material. With Electro-rheological fluid inkjet a specific designed ink is used which enters a plastic phase when an electric field is applied. Fluid flow is only enabled when the electric field is switched off, resulting in drop ejection (figure B.19).

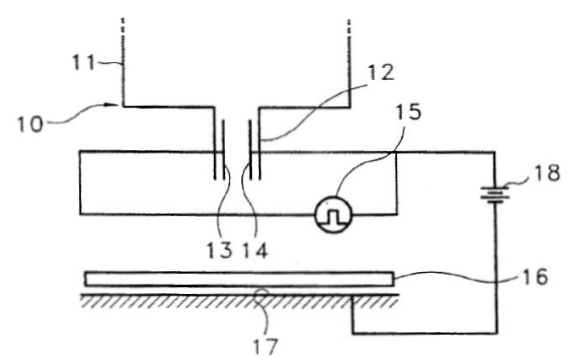

Figure B.19: Electro-rheological fluid inkjet; Samsung Electronics Co, 1996 [137]. 


\section{B.2.9 Thermal-rheological fluid inkjet}

In contrary to the above mentioned material dependent ejection mechanisms, thermalrheological fluid inkjet uses a constant supply pressure in contrary to an electric field to draw the droplets out of the nozzle. The supplied pressure is under normal temperature conditions not high enough to eject a droplet. If a nozzle is selectively heated, the viscosity of the ink will lower, enabling droplet ejection (figure B.20).

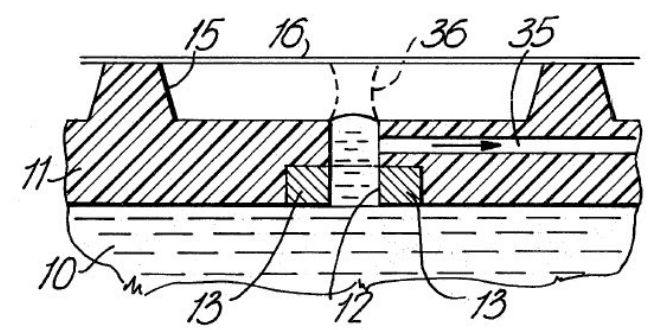

Figure B.20: Thermal-rheological fluid inkjet; Northern Telecom Limited, 1979 [138].

\section{B.2.10 Topspot microdrop ejector}

The topspot microdrop ejector has been designed for the generation of biochip microarrays. In this case it's not important that every nozzle can be controlled separately. The main priority is creating a system capable of ejecting material without having to alter its chemical composition. Three types of topspot systems can be described.

Inertial droplet release: Hereby the ejection of the droplet is caused by sudden stop of the nozzle holder. The momentum of the ink in the nozzle causes the fluid to be ejected (figure B.21).

Pneumatic droplet release: The content of the nozzle is ejected due to pressurization of the space above, resulting in drop ejection (figure B.22).

Direct liquid replacement: Similar to Pneumatic droplet release, however hereby an elastic monomer is used instead of gas pressure, resulting in better control (figure B.23). 


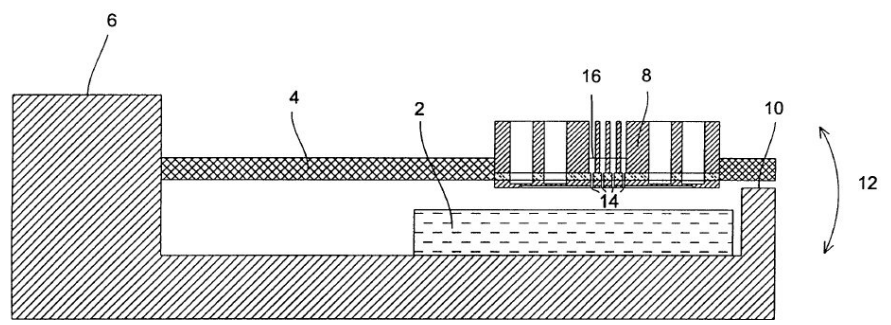

Figure B.21: Topspot microdrop ejector; Hahn-Schickard-gesellschaft für angewandte forschung, 2000 [139].

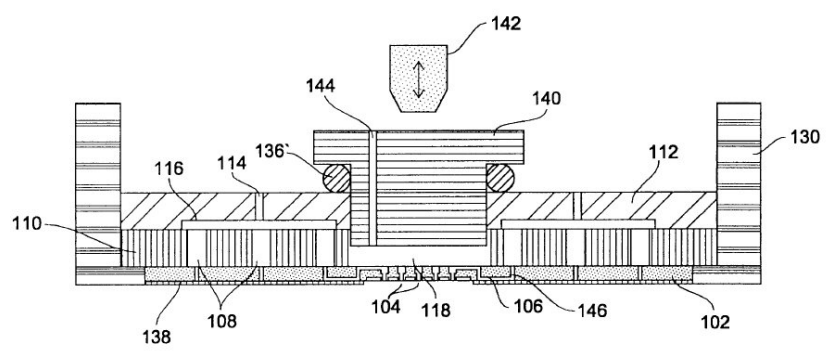

Figure B.22: Topspot microdrop ejector; Hahn-Schickard-gesellschaft für angewandte forschung, 2001 [140].

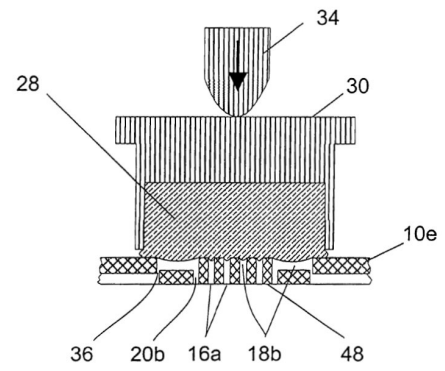

Figure B.23: Topspot microdrop ejector; Zengerle, 2004 [141]. 


\section{Boundary curves impact regimes}

In a phase diagram for different impact regimes several collision regimes can be distinguished (figure C.1):

$\diamond$ Coalescence (green)

$\diamond$ Reflective separation (blue)

$\diamond$ Stretching separation (yellow)

$\diamond$ Bouncing (red)

Several theoretical curves can be described between the different regimes. Equation C.1 describes curve A predicting the bouncing effect [54]. Equation C.8 describes curve B predicting the reflective separation effect at lower impact parameters [53] and equation C.12 or alternatively equation C.17 describes curve $\mathrm{C}$ predicting the separation at high impact parameters $[53,55]$.

$$
W e(I)=\frac{\Delta\left(1+\Delta^{2}\right) \cdot\left(4 \phi^{\prime}-12\right)}{\left(\chi_{l}\right) \cdot(\cos (\arcsin I))^{2}}
$$

with:

$$
\begin{gathered}
\phi^{\prime}=2\left(2 / \phi^{2}+1\right)^{-2 / 3}+\left(3 / \phi^{2}+1\right)^{1 / 3} \\
\phi=\frac{h}{r}
\end{gathered}
$$




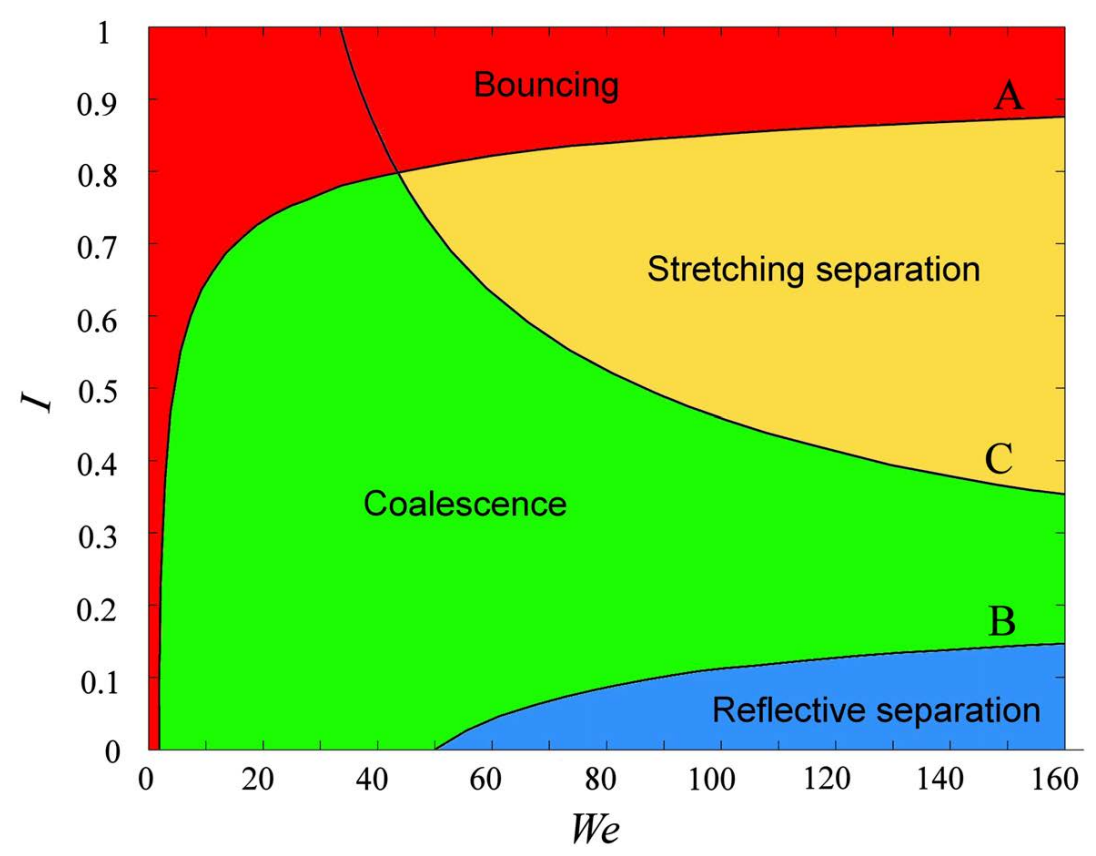

Figure C.1: Overview of impact regimes [54].

$$
\begin{array}{cc}
h=\frac{1}{2}\left(d_{l}+d_{s}\right)(1-I) & \\
\chi_{l}=\left(1-\frac{1}{4}(2-\tau)^{2}(1+\tau)\right) & \text { for } \omega>\frac{d_{l}}{2} \\
\chi_{l}=\frac{1}{4} \tau^{2}(3-\tau) & \text { for } \omega \leq \frac{d_{l}}{2}
\end{array}
$$

here $W e$ represents the Weber number, $I$ the non-dimensional impact parameter, $\Delta$ the diameter ratio, $\phi$ the shape parameter, $h$ the deformed droplet height, $r$ the deformed droplet radius, $\omega$ the width of the overlapping interaction region, $d_{l}$ and $s_{s}$ the diameter of the large respectively the small droplet

$$
W e(I)=-3\left[4\left(1+\Delta^{2}\right)-7\left(1+\Delta^{3}\right)^{\frac{2}{3}}\right] \cdot \frac{\Delta\left(1+\Delta^{3}\right)^{2}}{\left(\Delta^{6} \eta_{1}+\eta_{2}\right)}
$$


with:

$$
\begin{gathered}
\eta_{1}=2(1-\xi)^{2}\left(1-\xi^{2}\right)^{\frac{1}{2}}-1 \\
\eta_{2}=2(1-\xi)^{2}\left(\Delta^{2}-\xi^{2}\right)^{\frac{1}{2}}-\Delta^{3} \\
\xi=\frac{1}{2} I(1+\Delta) \\
W e(I)=\frac{4\left(1+\Delta^{3}\right)\left[3(1+\Delta)(1-I)\left(\Delta^{3} \phi_{s}+\phi_{l}\right)\right]^{\frac{1}{2}}}{\Delta^{2}\left[\left(1+\Delta^{3}\right)-\left(1-I^{2}\right)\left(\phi_{s}+\Delta^{3} \phi_{l}\right)\right]}
\end{gathered}
$$

with:

$$
\begin{array}{rlrl}
\phi_{s} & =1-\frac{1}{4 \Delta^{3}}(2 \Delta-\tau)^{2}(\Delta+\tau) & \text { for } h>\frac{1}{2} d_{s} \\
\phi_{s} & =\frac{\tau^{2}}{4 \Delta^{3}}(3 \Delta-\tau) & & \text { for } h<\frac{1}{2} d_{s} \\
\phi_{l} & =1-\frac{1}{4}(2-\tau)^{2}(1+\tau) & & \text { for } h>\frac{1}{2} d_{l} \\
\phi_{l} & =\frac{\tau^{2}}{4}(3-\tau) & \text { for } h<\frac{1}{2} d_{l}
\end{array}
$$

Or alternatively according to [55]

$$
I(W e)=\left(\frac{24}{5 W e}\right)^{\frac{1}{2}} \frac{\left(1+\Delta^{3}\right)^{\frac{11}{6}}}{(1+\Delta) \Delta^{\frac{5}{2}}}\left[1+\Delta^{2}-\left(1+\Delta^{3}\right)^{\frac{2}{3}}\right]^{\frac{1}{2}}
$$




\section{Summary}

The common denominator of this thesis is the quest for processing a wider range of materials using inkjet technology. One of the limiting factors in commercial available inkjet systems is the viscosity. None of these inkjet systems are able to process high viscosity fluids (above $100 \mathrm{mPa} \cdot \mathrm{s}$ ). To overcome this, a printhead has been developed, based on the first inkjet principle developed, namely continuous inkjet. Compared to a drop-on-demand system, where the actuation mechanism is responsible not only to form the droplets but also to force the material through the nozzle and accelerate the fluid, continuous technology splits the effort between the feed pressure and the actuation. The feed pressure presses the material through the nozzle and creates a flow of material (jet) while the actuation mechanism is required to start-up the breakup process to form the droplets. In this way a continuous stream of droplets is generated. In order to control the deposition of material in time and space droplet selection is needed. In this thesis the development phases of the complete print system are described, from the material feed mechanism towards various ways to select droplets.

\section{Droplet creation}

For the creation of droplets of a high viscous fluid it is not straightforward to induce a vibration intense enough to form droplets even when using a continuous inkjet principle. Several layouts can be found in literature, all limited however, to the jetting of low viscous fluids. The principle described in this thesis uses a vibrating element which is placed just above the nozzle to induce the vibration as close to the jet as possible to enforce droplet formation [17]. The effect of the increased viscosity on the operating window for stable droplet formation is assessed [39-44]. A model is developed to assess the performance of the printhead depending on chosen system parameters (dimensions, viscosity, density), showing the importance of the small gap between vibrating element and the nozzle.

The pressures necessary to process the ink with increased viscosity not only gives challenges for the droplet generation, it also requires the development of a different fluid supply. This should be capable of delivering the required flow rate at pressures 
of up to 200 bars. With conventional continuous inkjet systems a pressure controlled material supply is used. When using high viscous materials frequently a higher startup pressure is observed as compared to the system's working pressure. Therefore a continuous-flow ink supply is developed [18] to allow for easy start-up of the system. The usage of such a flow controlled supply results in a more material independent system layout; the generated droplet size can be calculated from the set material feed and actuating frequency, independent of fluid viscosity, operating pressure or nozzle size. This results in more stable operation, regardless of fluctuations in material properties. Experimentally the operating range of the printhead is obtained by observing the breakup process using a stroboscopic illumination system and a camera. The chosen design results in a system capable of jetting a wide variety of different materials within a broad processing window. Viscosities up to $500 \mathrm{mPa} \cdot \mathrm{s}$ can be used. For a 200 $\mathrm{mPa} \cdot \mathrm{s}$ test fluid the range of operation is described in detail. From the experiments it can be derived that compared to jet and droplet formation controlled by a vibrating nozzle, the displacement of the vibrating member of the present design has been reduced with a factor 80 , still achieving equal droplet breakup, giving an impression of the increased performance of the design. Depending on the specific application several alternative designs are proposed, from vibrating nozzle plates utilizing a focussing member close to the nozzle [20], a system replacing the piezo actuation with a rotating notched disc $[19,21]$, to a pressure independent actuator layout especially interesting when moving towards larger multi nozzle systems [22].

\section{Droplet selection}

Generating a continuous stream of droplets alone does not result in a functional printing system; for this purpose a selection mechanism is required. The use of conventional electrostatic deflection systems seems obvious, but often material modifications are needed. Although a method is proposed to increase the conductivity of the material with minor modifications [23], alternatives are described without the need of any material adaptation, from droplet collision [24], to air-jets deflecting undesired droplets [25].

Ballistic deflection: For the investigation of the collision (ballistic) deflection system, an experimental setup has been built where a drop-on-demand printhead ejects droplets which are timed such that they collide with the continuously formed droplet stream. Several collision regimes are observed depending on the chosen impact number; bouncing, coalescence, stretching separation and reflective separation. The bouncing regime is preferred since no material is exchanged between both droplets during impact, maintaining two separate fluid streams. The experiments are performed using two different colliding droplets, with not only different materials, but 
also different sizes. A tri-ethylene glycol droplet with a diameter of $110 \mu \mathrm{m}$ is generated by the continuous inkjet, and a water droplet with a diameter of $55 \mu \mathrm{m}$ is generated by the drop-on-demand printhead. The experimental results are compared with literature describing either equal sized droplets with different material types [56] or equal material type droplets with different sized droplets [54]. The differences observed are described. Only few collision outcomes were sufficient to achieve sufficient deflection to create a functional system (set at $2^{\circ}$ jet deviation). Almost all of these results were found in the stretching separation regime, resulting in undesired fluid transfer between both droplets. The system is functional on lab scale, but its complexity and the material transfer between both fluid streams limits its industrial applicability.

Air-jet deflection: Therefore an alternative deflection mechanism is designed using an air-jet to deviate the droplets from their initial path. The main challenge with this specific layout is to create a system capable of selecting only one single droplet at the time. In this case the continuous air jet has to be moved in the droplets path in the time gap in-between two passing droplets. Experiments are performed to asses the deflection angle that can be obtained using airflows from small needles. Using needles with an inside diameter of $100 \mu \mathrm{m}$ and $150 \mu \mathrm{m}$ it is possible to achieve deflection angles of up to $7.5^{\circ}$ (up to almost $16^{\circ}$ if the droplets are allowed to disintegrate). These needle sizes allow $29 \mu \mathrm{s}$ (100 $\mu \mathrm{m}$ air-jet) or $24 \mu \mathrm{s}(150 \mu \mathrm{m}$ air-jet $)$ setup time for the needle movement mechanism when deflecting 110 micron droplets generated at $20 \mathrm{kHz}$ passing with a velocity of $10 \mathrm{~m} / \mathrm{s}$. A piezo-actuated movement mechanism is described obtaining even $20 \mu$ s setup time by using input shaping of the control signal. The systems performance is experimentally validated and showed stable performance; special attention, however, is needed for the cooling of the deflection system to prevent overheating. The high achievable deflection angles give opportunities to decrease the distance between nozzle and substrate, giving room for increasing the systems overall accuracy. If electrostatic deflection can be used this remains preferable due to the lack of moving parts, but the air-jet deflection offers a good alternative for non-conducting liquids.

\section{Alternative applications}

The initial reason for the development of a printing system capable of processing higher viscosities came from the desire to deposit functional materials to create a 3D manufacturing process, allowing layer-wise fabrication of end products. The system developed appears to have a much broader range of application areas.

Powder production: For example in the field of powder processing the technology allows monodisperse powders to be created [26]. The relative span of the particle 
size distribution from 1-2 for a powder generated by a conventional process can be reduced to $0.6-0.7$ for a powder generated using inkjet technology. The monodispersity of the droplets does not only result in increased powder properties, it also enables more exact dosing of drying air resulting in an increase in energy efficiency. The printhead is even able to process a material feed containing high concentrations of solids resulting in an even higher overall system performance. The absence of fines leads to a more economic process due to a reduction of exhaust air filtration. The created powders are different in nature, compared to conventional agglomerated powders containing relatively large amounts of enclosed air. The resulting powders are spherical without air enclosures resulting in high density powders. For a model material consisting of maltodextrin a 50\% density increase is measured, for a creamer based model material a density increase of $30 \%$ is realized compared to powders created using a swirl flow nozzle. The process handles the materials with care, allowing processing of sensitive emulsions.

The process, however, differs in a primary aspect from conventional spray drying. Under normal circumstances the droplets generated from the spray nozzle are orders smaller than the final product; due to air flow in the spray tower agglomeration of the droplets in flight is promoted, resulting in the final powder size. When using inkjet technology, monodisperse droplets are generated right away. This means that the air flow in the spray tower must be designed such that agglomeration is prevented. A second challenge is directly linked. To end up with a comparable dried particle size the initial generated droplets are larger, requiring a longer drying time, frequently not achievable in a conventional setup. It can, however, be argued whether a similar particle size is required. Since the generated powder has a different morphology, the required particle size to achieve the required behavior (e.g. solubility) probably differs as well. Therefore more elaborate investigation of generated powders and occurring drying effects is necessary. To that end a dedicated test facility is fabricated to continue research on this topic.

Encapsulation: A second application makes use of the directionality and controlled speed of the droplets enabling a novel encapsulation process. By propelling the generated droplets through a liquid screen, encapsulates can be made [27]. With this new processing technology it is possible to create encapsulates with a hydrophobic material contained in a hydrophilic shell, as well as the opposite, a hydrophilic payload inside a hydrophobic shell. Both process flows are generated separately, allowing for different initial parameters (temperature, speed), enabling creation of temperature sensitive payloads encapsulated in for instance solidifying/heated wax. Also reactive systems can be combined due to the physical distance between the printhead and the liquid jet generator. The encapsulation process shows opportunities for scale-up al- 
lowing direct usage in pilot scale spray dryers, by combining the technology with a multi nozzle printhead.

The smaller the encapsulates need to be, the smaller the required initial droplets, making it more difficult for the droplet to break through the screen. Increasing the initial velocity might provide opportunities [27] or methods to reduce the screen's thickness [28]. The usage of a liquid screen to create encapsulates is not limited to inkjet generated droplets. Alternatives are described propelling existing particles through such a screen [29].

\section{Metal printing}

Layer-wise fabrication of products allows for the creation of multi-material or even graded structures. Integration of electronics within a product would lead to new opportunities. To make this a reality it is necessary to deposit electric connections. Therefore two direct metal deposition principles are described; pyrolitic metal printing [142] and direct metal printing [143].

Pyrolitic metal printing: The first process creates droplets of metal salt solutions which are pyrolised in flight towards the substrate. Since quite a long distance is required to evaporate and pyrolise the droplets, means are necessary to maintain positioning accuracy on the substrate. Therefore the droplets are focussed aerodynamically [31] achieving a deposition accuracy of 5 micron. Unfortunately, the focussing effect at this stage only functions at reduced temperatures. When the temperature is increased and pyrolises takes place the aerodynamic focussing accuracy decreases, probably by fragmentation of the generated droplets during the pyrolises process. Further research is required to tune the pyrolises phase to maintain monodisperse droplets allowing the focussing step to work optimally. Creation of conductive tracks using the technology proved functional albeit at reduced accuracies, resulting in track widths of approximately $300 \mu \mathrm{m}$.

Direct metal printing: The second process, direct metal printing, deposits metal droplets directly from the melt. Therefore a printhead is developed capable of operating at $1140^{\circ} \mathrm{C}$ allowing deposition of Feingold. Due to the expensive nature of the material used a drop-on-demand principle is applied enabling deposition of $120 \mu \mathrm{m}$ droplets using a $70 \mu \mathrm{m}$ nozzle. A combination with a software controlled XY-stage allows images to be printed. To reduce the volume which should be held at these extreme temperatures a material feeding mechanism is described allowing the amount of molten metal to be reduced to a minimum [32].

\section{$\mathbf{3}^{\text {rd }}$ industrial revolution?}

Finally an overview is given [144] of the challenges occurring when implementing 
the developed inkjet technology and creating a 3D layer-wise production system. Combining multiple materials into one single product opens new opportunities. The described process is used in one of the first systems capable of generating gradients in material properties within one product. This ability sets the need for a radically new way of product development and design. One no longer has to think in terms of limitations imposed by current production systems and materials, but can think unrestricted about what products we want, depending on the demands and needs we have. One can only get an impression of possibilities this might lead to. Until now only nature was able to generate these structures. Now production technology is starting to catch up. 


\section{Samenvatting}

Dit proefschrift beschrijft de zoektocht naar het verwerken van een breder scala aan materialen met behulp van inktstraaltechnologie. De beperkende factor van commercieel verkrijgbare inktstraalsystemen is de maximaal verwerkbare viscositeit. Om de huidige grenzen ten aanzien van viscositeit te verleggen, is een printkop ontwikkeld, gebaseerd op één van de oudste inktstraal principes, namelijk gebaseerd op een continue inktstraal. Vergeleken met een één-druppel proces, waar het activatie mechanisme verantwoordelijk is voor het vormen van de gevraagde druppel, het persen van de vloeistof door de spuitmond en het versnellen van de druppel, worden deze functies gesplitst bij een continu inktstraal systeem. Daarbij wordt door een constante toevoerdruk het materiaal door een spuitmond geperst waardoor een snelle vloeistofstraal ontstaat, terwijl het activatie mechanisme enkel zorgt voor het opbreken van de straal. In dit proefschrift worden de ontwikkelingsfasen van het complete printsysteem beschreven, van het materiaal toevoermechanisme tot en met verschillende druppelselectie mechanismen.

\section{Druppelvorming}

Voor het maken van druppels van een hoog viskeuze vloeistof met behulp van continue inktstraaltechnologie is het nodig om een trilling te genereren die intens genoeg is om de viskeuze vloeistofstraal op te breken. Er zijn weliswaar verschillende uitvoeringsvormen van opbreekmechanismen in de literatuur te vinden, echter allemaal beperkt toepasbaar als het gaat om het verwerken van viskeuzere vloeistoffen. Het in dit proefschrift beschreven principe maakt gebruik van een trillend element, zo dicht mogelijk boven de spuitmond gepositioneerd, zodat in de vloeistofstraal een beweging op gang komt om de opbreking op te wekken [17]. Op basis van gegevens uit de literatuur wordt onderzocht of een stabiel opbreekgedrag bij hoog viskeuze materialen mogelijk is [39-44]. Vervolgens wordt een model beschreven dat de verwachte druk en debietfluctuatie in de spuitmond beschrijft om zo een indruk te krijgen van de prestaties van het systeem. Hieruit blijkt nogmaals het belang van de kleine afstand tussen het trillend element en de spuitmond.

De hoge druk die nodig is om de vloeistof met hoge viscositeit te kunnen ver- 
werken vraagt om de ontwikkeling van een aangepast vloeistoftoevoersysteem. Bij conventionele continue inktstraalsystemen wordt een drukgestuurd systeem gebruikt om een straal met constante snelheid te realiseren. Bij het gebruik van hoog viskeuze materialen is vaak een hogere opstart druk nodig vergeleken met de werkdruk onder bedrijfsomstandigheden. Daarom is een toevoersysteem ontwikkeld dat gebaseerd is op het genereren van een constante volumestroom [18] waardoor een eenvoudige opstart van het systeem mogelijk wordt. Het gebruik van debietsturing resulteert in een materiaal onafhankelijk systeem; de druppelgrootte kan worden berekend aan de hand van de ingestelde volumestroom gecombineerd met de gekozen activatie frequentie, onafhankelijk van viscositeit, werkdruk en grootte van de spuitmond.

Het werkgebied van de printkop is experimenteel bepaald door het opbreekproces met behulp van stroboscopische belichting in beeld te brengen.

Het uiteindelijke ontwerp (een trillend element dicht bij de spuitmond, aangestuurd door een piëzo element) resulteert in een systeem dat het verwerken van een groot aantal verschillende materialen over een breed werkgebied mogelijk maakt. Het verwerken van materialen met viscositeiten tot $500 \mathrm{mPa} \cdot \mathrm{s}$ bij verwerkingstemperatuur is mogelijk. Als voorbeeld wordt het werkgebied van een testvloeistof met een viscositeit van $200 \mathrm{mPa} \cdot \mathrm{s}$ uitgebreid beschreven. Uit experimenten kan worden afgeleid dat, in vergelijking met een longitudinaal bewegende inktstraal pomp, de noodzakelijke verplaatsing van het trillend element kan worden gereduceerd met een factor 80 bij gelijke opbreking van de druppels, wat een indruk geeft van de verbeterde prestaties van het ontwerp.

Afhankelijk van de specifieke toepassing kan het wenselijk zijn om een alternatieve uitvoeringsvorm te kiezen. Hiertoe zijn drie alternatieve ontwerpen beschreven. Het eerste alternatief bevat een trillende spuitmond waarbij de actuatie wordt versterkt door het plaatsen van een stilstaand element vlak voor de spuitmond [20]. Het tweede alternatief is een systeem waarbij het piëzo element wordt vervangen door een roterende reliëf schijf, die net als de piëzo in staat is vlak voor de spuitmond een verstoring te veroorzaken $[19,21]$. Het laatste beschreven alternatief bevat een drukonafhankelijke actuator. Dit systeem is interessant bij de opschaling naar grotere systemen met meerdere spuitmonden [22].

\section{Druppelselectie (middels afbuiging)}

Het genereren van een continue stroom van druppels leidt nog niet tot een functioneel printsysteem; daar is een selectiemechanisme voor nodig. Bij bestaande continue inktstraalsystemen wordt hiervoor gebruik gemaakt van elektrostatische afbuiging, waarbij de druppels selectief van een lading worden voorzien. Deze geladen druppels worden vervolgens door middel van een elektrostatisch veld afgebogen in 
de richting van een specifieke plek op het substraat. Indien er geen afbeelding wordt geprint, worden de druppels in een afvoergoot gericht voor recirculatie. Het gebruik van een dergelijk afbuigsysteem ligt voor de hand, maar is helaas vaak niet mogelijk zonder wijzigingen in de materiaalformulering aan te brengen. Hoewel een werkwijze wordt voorgesteld om de geleidbaarheid van het materiaal te vergroten met kleine aanpassingen [23], worden alternatieven beschreven voor het selecteren van druppels, waarbij geen aanpassing van het materiaal noodzakelijk is, zoals het gebruik van druppelbotsing [24], en luchtstralen om ongewenste druppels af te buigen [25].

Afbuiging door middel van botsing: Voor het onderzoek naar het (ballistische) botsing afbuigsysteem, waarbij druppels uit een continu inktstraal systeem worden weggeschoten door druppels uit een één-druppel systeem, is een experimentele opstelling gebouwd. De één-druppel printkop genereert druppels op een zodanig tijdstip dat deze botsen met specifiek geselecteerde druppels uit de continue stroom druppels. Afhankelijk van gekozen parameters, zijn verschillende soorten botsingen onderzocht; "botsen", "stabiel samensmelten, "instabiel samensmelten gevolgd door opbreken" en "instabiel samensmelten gevolgd door terugkaatsen". Hiervan heeft "botsen" de voorkeur omdat er dan geen materiaal wordt uitgewisseld tussen beide druppels, waardoor de vloeistofstromen gescheiden blijven. Botsexperimenten zijn uitgevoerd met twee druppels van verschillende materialen en van verschillende afmetingen. Tri-ethyleen glycol druppels met een diameter van $110 \mu \mathrm{m}$ worden door de continue inktstraal printkop gegenereerd en één druppel water met een diameter van $55 \mu \mathrm{m}$ wordt door de één-druppel printkop afgevuurd. De experimentele resultaten zijn vergeleken met de literatuur, waarin zowel botsingen tussen druppels van gelijke afmetingen en verschillende materialen beschreven worden [56], als van verschillende afmetingen en gelijke materialen [54]. Uit de botsexperimenten resulteerden slechts enkele bruikbare resultaten (waarbij een minimale afbuiging van $2^{\circ}$ als bruikbaar is gedefinieerd). Deze vonden bijna allemaal plaats in het gebied van "instabiel samensmelten gevolgd door opbreken", resulterend in een ongewenste overdracht van vloeistof tussen beide druppels. Het systeem blijkt te werken in het laboratorium, maar de complexiteit en de overdracht van materiaal tussen beide vloeistofstromen maken de industriële toepasbaarheid twijfelachtig.

Afbuiging door middel van luchtstralen: Daarom is een alternatief afbuigingsmechanisme ontworpen, dat gebruik maakt van een luchtstraal om de druppels weg te blazen uit hun oorspronkelijke pad. De belangrijkste uitdaging in deze specifieke uitvoering is het creëren van een systeem dat in staat is om slechts een enkele druppel per keer te selecteren. De beschikbare tijd voor de verplaatsing van de continue luchtstraal in het pad van de druppels is slechts beperkt tot het tijdsinterval tussen twee passerende druppels, aangezien anders de overige druppels worden beïnvloed. De 
hoge frequenties waarmee druppels worden gegenereerd, resulteert in de noodzaak van een extreem snelle beweging. De beschikbare tijd is tevens afhankelijk van de afmeting van de naald (waar de lucht doorheen wordt geblazen); hoe kleiner de gekozen naald, des te meer tijd er beschikbaar is voor de beweging, echter des te minder het afbuigende effect van de luchtstraal. Er zijn experimenten uitgevoerd om de afbuighoek te beoordelen die kan worden verkregen met behulp van luchtstromen uit kleine naalden. Met behulp van naalden met een binnendiameter van $100 \mu \mathrm{m}$ en $150 \mu \mathrm{m}$ is het mogelijk om afbuighoeken te behalen van tot wel $7,5^{\circ}$ (tot bijna $16^{\circ}$ als de druppels mogen desintegreren). Deze naaldafmetingen staan een insteltijd voor het naald bewegingsmechanisme toe van $29 \mu \mathrm{s}$ (100 $\mu$ s luchtstraal) respectievelijk $24 \mu$ s (150 $\mu$ s luchtstraal) bij het afbuigen van $110 \mu$ m druppels, welke zijn gegenereerd met een frequentie van $20 \mathrm{kHz}$ en passeren met een snelheid van $10 \mathrm{~m} / \mathrm{s}$. Een piëzo aangedreven bewegingsmechanisme is beschreven dat zelfs een insteltijd van $20 \mu$ s mogelijk maakt door het ingangssignaal van te voren af te stemmen op de te verwachten beweging van de naald.

De werking van het systeem is experimenteel bevestigd en functioneert stabiel, maar er is speciale aandacht nodig voor het koelen van het afbuigsysteem, om oververhitting te voorkomen. De grote afbuighoeken die te behalen zijn geven mogelijkheden om de afstand tussen spuitmond en substraat te verkleinen, wat ruimte biedt voor het vergroten van de complete nauwkeurigheid van het systeem. Toch heeft het gebruik van elektrostatische afbuiging, vanwege het ontbreken van bewegende onderdelen, de voorkeur indien toepasbaar, maar de luchtstraal afbuiging biedt een goed alternatief voor niet-geleidende vloeistoffen.

\section{Alternatieve toepassingen}

De voornaamste reden voor de ontwikkeling van een printsysteem dat hoog viskeuze vloeistoffen kan verwerken, is de wens om functionele materialen te kunnen verwerken en hierdoor een laagsgewijs productieproces te realiseren dat directe fabricage van eindproducten mogelijk maakt. Het ontwikkelde systeem lijkt een veel breder scala aan toepassingsgebieden te hebben. Enkele voorbeelden zullen nader worden belicht.

Poederproductie: Bijvoorbeeld op het gebied van poederproductie kan het gebruik van de in dit proefschrift beschreven techniek ervoor zorgen dat monodisperse poeders kunnen worden gemaakt [26]. Bij een conventioneel sproeidroog proces wordt het te drogen materiaal door een spuitmond in de droogkamer verneveld, waarna de gegenereerde druppels door droge lucht worden omgevormd tot poeders. De gebruikte spuitmond produceert een turbulent sproeibeeld. De brede intiële spreiding en agglomeratie van de gegenereerde deeltjes resulteert in een poeder met 
een grote spreiding aan deeltjesgroottes. Door gebruik te maken van inktstraaltechnologie kan de druppelgrootte exact worden ingesteld waardoor de spreiding bijna kan worden gehalveerd. De monodispersiteit van de druppels leidt niet alleen tot verbeterde poedereigenschappen, maar maakt tevens een nauwkeurige dosering van drooglucht mogelijk hetgeen resulteert in een betere energie efficiëntie. De printkop is zelfs in staat om een materiaal te verwerken met hoge concentraties vaste stof waardoor minder oplosmiddel in de droogtoren hoeft worden te verdampt, resulterend in een nog hogere energie efficiëntie van het totale droogsysteem. Daarnaast leidt de afwezigheid van extreem kleine deeltjes tot een economisch voordeliger proces aangezien de systemen voor filtratie van de afgevoerde lucht kunnen worden beperkt. De poeders zijn anders van aard, in vergelijking met conventionele geagglomereerde poeders die relatief veel ingesloten lucht bevatten. De met inktstraaltechnologie gemaakte poederdeeltjes zijn bolvormig zonder ingesloten lucht en hebben een hoge dichtheid. In vergelijking met conventionele poeders is, bij een testmateriaal bestaande uit maltodextrine, een dichtheidstoename van 50\% waargenomen, en bij een creamer $30 \%$. Het inktstraal proces veroorzaakt weinig mechanische en thermische belasting, waardoor verwerking van gevoelige emulsies mogelijk wordt.

Het proces verschilt in nog een belangrijk opzicht van conventioneel sproeidrogen. Onder normale omstandigheden zijn de druppels gegenereerd uit de spuitmond enkele ordes kleiner dan het eindproduct. Als gevolg van de luchtstroom in de sproeitoren wordt agglomeratie van de druppels tijdens hun vlucht bevorderd, wat leidt tot de uiteindelijke grootte van de poederdeeltjes. Bij het gebruik van inktstraaltechnologie worden direct monodisperse druppels geproduceerd. Dit betekent dat de luchtstroom in de sproeitoren zodanig moet worden ontworpen dat agglomeratie juist wordt voorkomen. De druppels die door de inktstraal gemaakt worden, moeten dan wel groter zijn, om op een gelijke grootte van de poederdeeltjes uit te komen dan druppels die bij het conventionele proces door een sproeispuitmond worden geproduceerd voor agglomeratie. Dit vereist wel een langere droogtijd, wat vaak niet haalbaar is met een conventionele drooginstallatie. Men kan zich echter afvragen of de gewenste deeltjesgrootte bij het inktstraal proces wel gelijk moet zijn aan die van het conventionele proces. De gewenste deeltjesgrootte is het gevolg van gewenste producteigenschappen van het poeder (zoals oplosbaarheid). Het gegenereerde poeder heeft een andere morfologie, en gedraagt zich bij gelijke afmeting anders, wat leidt tot een andere deeltjesgrootte om dezelfde producteigenschappen te realiseren. Om dit exact te bepalen is meer onderzoek nodig aan de poeders die met een inktstraal geproduceerd worden en de daarvoor optimale droogeffecten. Een speciale proefopstelling wordt gemaakt om het onderzoek voort te zetten.

Encapsulatie: Een tweede voorbeeld maakt gebruik van de richting en de snelheid 
van de druppels hetgeen een nieuw encapsulatie proces mogelijk maakt. Door de gegenereerde druppels door een vloeistofscherm heen te schieten, kunnen capsules worden gemaakt [27]. Het is mogelijk om capsules te maken van een waterafstotend materiaal in een hydrofiel omhulsel, maar ook het tegenovergestelde, namelijk een hydrofiel materiaal in een waterafstotend omhulsel. Beide processtromen worden afzonderlijk gegenereerd waardoor het mogelijk is om verschillende procesparameters te gebruiken (temperatuur, snelheid). Zo is het maken van capsules van temperatuurgevoelig materiaal in bijvoorbeeld verwarmde/stollende was mogelijk. Ook kunnen, door de aanwezigheid van een zekere fysieke afstand tussen de printkop en de vloeistofscherm generator, reactieve systemen worden gecombineerd. Het encapsulatie proces leent zich voor opschaling; men kan zich een uitvoering voorstellen met veel spuitmonden, waardoor gebruik hiervan in bijvoorbeeld een sproeidroger mogelijk is. Hoe kleiner de capsules moeten zijn, des te kleiner de vereiste initiële druppels; het doorbreken van het scherm wordt voor een zeer kleine druppel problematisch. Het verhogen van de beginsnelheid [27] of methoden om de dikte van het scherm te reduceren [28] kunnen oplossingen bieden. Het gebruik van een vloeistofscherm om capsules te maken is niet beperkt tot druppels gegenereerd via een inktstraal proces. Alternatieven worden beschreven waarbij bestaande deeltjes op snelheid worden gebracht en door een dergelijk scherm worden geschoten [29].

\section{Metaalprinten}

Laagsgewijze fabricage van producten maakt realisatie mogelijk van producten die bestaan uit meerdere materialen, zelfs producten met een verloop van het ene materiaal naar het andere. De integratie van elektronica in een product leidt tot nieuwe kansen. Om dit te realiseren is het noodzakelijk elektrische aansluitingen te kunnen maken. Daartoe zijn twee metaal verwerkingsprincipes beschreven; pyrolitisch metaal printen [142] en direct metaal printen [143].

Pyrolitisch metaal printen: Het eerste proces creëert druppels van zoutoplossingen van metaal die worden gepyrolyseerd op weg naar het substraat. Aangezien een vrij grote afstand nodig is voor het verdampen en pyroliseren van de druppels, zijn middelen noodzakelijk om de nauwkeurigheid van de landingspositie van de druppel op het substraat te behouden. Daarom worden de druppels gericht door middel van een convergerende gasstroom [31] waardoor een plaatsingsnauwkeurigheid bereikt wordt van $5 \mu \mathrm{m}$. Helaas functioneert het focusserend effect slechts bij lagere temperaturen. Wanneer de temperatuur wordt verhoogd en pyrolise plaatsvindt, neemt de precisie van de aerodynamische focussering af, waarschijnlijk door fragmentering van de druppels tijdens het pyroliseproces. Verder onderzoek is nodig om de monodispersiteit van de druppels tijdens de pyrolisefase te behouden, waardoor de 
focusseringsstap optimaal kan gaan werken. Het is mogelijk om geleidende sporen te maken met deze techniek, zij het met verminderde nauwkeurigheid, resulterend in spoorbreedten van ongeveer $300 \mu \mathrm{m}$.

Direct metaal printen: Het tweede proces, direct metaal printen, verwerkt metaaldruppels rechtstreeks vanuit gesmolten toestand. Hiervoor is een printkop ontwikkeld die kan werken bij een temperatuur van $1140^{\circ} \mathrm{C}$ wat de verwerking van Feingold mogelijk maakt. Als gevolg van de hoge kostprijs van het gebruikte materiaal wordt een één-druppel principe toegepast in plaats van een continue straal systeem $(120 \mu \mathrm{m}$ druppels uit een $70 \mu \mathrm{m}$ spuitmond). In combinatie met een software gestuurde XYpositioneertafel wordt het mogelijk om afbeeldingen te printen. Een groot toevoerreservoir voor het materiaal is, in het kader van energie efficiëntie, bij deze extreme temperaturen ongewenst, daarom wordt een toevoermechanisme beschreven waarbij de hoeveelheid gesmolten metaal tot een minimum kan worden beperkt [32].

\section{$3^{e}$ industriële revolutie?}

Tot slot wordt een overzicht gegeven [144] van de uitdagingen die zich voordoen bij de implementatie van de ontwikkelde printtechnologie en het creëren van een 3Dlaagswijs productiesysteem. Het combineren van meerdere materialen in een enkel product levert nieuwe mogelijkheden. Het beschreven proces is één van de eerste systemen waarmee in elkaar overlopende materiaaleigenschappen gerealiseerd kunnen worden in één product, door sommigen ook wel de $3^{e}$ industriële revolutie genoemd [84]. Dat vraagt om een radicaal nieuwe manier van productontwikkeling en -ontwerp. Er moet niet langer gedacht worden in termen van beperkingen, opgelegd door de huidige productiesystemen en materialen, maar er kan zonder beperkingen gedacht worden over de producten die we willen, afhankelijk van de eisen en behoeften die we hebben. Tot nu toe was alleen de natuur in staat om dergelijke structuren te genereren. $\mathrm{Nu}$ is de productietechnologie begonnen aan een inhaalslag. 


\section{Acknowledgements}

The research, described in this thesis, is carried out at the Equipment for Additive Manufacturing Department of TNO in Eindhoven, in close cooperation with the Physics of Fluids research group of the faculty of Science and Technology of the University of Twente. I want to thank all the people who have contributed to this research. This thesis is the result of ten years of my work at TNO, in the field of printing research and development, added up this last year with scientific legitimacy. As a pleasant side-effect, TNO and the University of Twente are working closer together on this field, resulting in synergy, speeding up technology development.

I am very thankful to my promotors, Frits Dijksman and Detlef Lohse. Frits, for his guidance and encouragement, he was not only a mentor and sparring partner for me, but also an inspirator, especially on the field of modelling technique. I enjoyed the stimulating meetings we had, mostly in his living room in Weert. I am also very grateful to Detlef, for his support, input and for his accurate feedback. The exposed interest of both Frits and Detlef were of great support to me. I want to thank TNO management for providing the chance to carry out this research and to write this thesis, especially Ardi Dortmans and Egbert-Jan Sol, in cooperation with Machteld de Kroon and Erwin Meinders. Not to forget Pierre Senster who urged me to reconsider, but still providing the flexibility for me to start up the work for this thesis.

I would like to extend my admirations to those, whom I worked with the past ten years, especially Leon Brouwers and André Rijfers with whom I have numerous discussions and without them the work described could not have been realized. But also Frits Feenstra for his continuous backup and support in finding funding possibilities and giving the development freedom in his projects. Also Gerben Pieterse for his input and feedback on the modelling of the printhead, as well as Robin Koldeweij whose help with programming was very welcome, and of course Guus Biemans for his experimental work. I am also indebted to graduate students and students from the Stan Ackermans institute for their contribution to several parts of the research, Peter Nouws and Eduardo Ribeiro for their work in the field of spray drying and Thomas van Gils for his work with respect to droplet collisions. I would like to thank 
my colleagues at TNO and others (outside TNO) with whom I did research, and wrote patent applications and publications. Especially I would like to mention Anton Aulbers, Kjeld van Bommel, Ronald Boot, Henk van Deventer, Pepijn van den Eijnden, Jacco Eversdijk, Guido Gubbels, Maarten van Lent, Jasper Michels, Gerrit Oosterhuis, Jeroen Smeltink, Frits Verhoeven, Bart van de Vorst, Fred de Vrede and Michiel Willemse. But also Jörg Fischer-Buehner and Peter Hofmann from Indutherm Erwaermungsanlagen $\mathrm{GmbH}$, Ulrich Klotz from Forschungsinstitut Edelmetalle \& Metallchemie, Gerd Wagner from Reishauer GmbH, and Pascal Pierron from Ardeje. They made it possible to use the information from the patent applications and articles in this thesis. And of course Johannes van Melle for the numerous discussions during the process of filing the patent applications.

I am thankful to Albert Poortinga, Frank Jeurissen, Jasper Vollenbroek and Tom van Hengstum from Royal FrieslandCampina, for the inspiration during several meetings and for the fellowship that made it possible to translate technique into practice.

I also want to thank Tessa ten Cate and Eric Craenmehr for their extended work in the field of encapsulation, Johan Keern for his practical help whenever materials needed formulating. And of course everybody from the work and modelshop; without their work it would have been a desktop study.

The list of names is not exhaustive and I apologize to those whose names are unacknowledged.

Thanks to my parents and sister for their continuous trust. Finally I thank my wife and kids for their encouragement, understanding and support during many hours of their time I consumed, which I could not spend with them. I thank everybody for their best wishes. 


\section{About the author}

René Jos Houben was born on May 26th, 1978, in Geldrop, the Netherlands. He received his high school education (VWO) at Jeanne d'Arc College in Maastricht and graduated in 1996. In the same year he started studying Mechanical Engineering at the Eindhoven University of Technology. He conducted his practical assignment (development of a dimethyl ether pre-pump) and graduating assignment (direct ink-jet printing of ceramic filled polymer as rapid fabrication technique) both at TNO (resp. Delft and Eindhoven), and obtained his Msc. in December 2001. After his graduation he started working at TNO in Eindhoven in the field of rapid manufacturing, focussing on printhead development and deposition of non conventional materials. Applications ranged far beyond the scope of 3D manufacturing, from printing mechanisms for decorating glass building façades to spray drying of powders. In April 2011 he had (co-)written more than 25 patents in this field and started to report about his work of the past ten years in this thesis, next to his regular job at TNO. The thesis has been realized in cooperation with the Physics of Fluids Group at the University of Twente under guidance of prof. dr. ir. Frits Dijksman and prof. dr. rer. nat. Detlef Lohse. 

ONE OF THE LIMITING FACTORS IN COMMERCIAL AVAILABLE INKJET SYSTEMS IS THE MAXIMUM ALLOWABLE INK VISCOSITY. TO OVERCOME THIS, A PRINTHEAD HAS BEEN DEVELOPED, BASED ON CONTINUOUS INKJET TECHNOLOGY. IN THIS THESIS THE DESIGN PHASES OF THE COMPLETE PRINT SYSTEM ARE DESCRIBED. SEVERAL ASPECTS ARE DISCUSSED ALL THE WAY FROM THE MATERIAL SUPPLY MECHANISM, THE DROPLET GENERATION MECHANISM ITSELF, TOWARDS MEANS AND METHODS TO CONTROL THE DROPLET LANDING POSITION WITHOUT IHE NEED FOR MATERIAL ADAPTATION. THEREAFTER SEVERAL APPLICATION AREAS ARE DESCRIBED, FROM POWDER GENERATION TOWARDS NOVEL ENCAPSULATION METHODS. FOR THE REALIZATION OF FUNCTIONAL $3 D$ ELECTRONICS, USING ADDITIVE MANUFACTURING TECHNIQUES, IT IS REQUIRED TO CREATE CONDUCTIVE TRACKS WITHOUT ADDITIONAL HIGH TEMPERATURE PROCESSING STEPS. TO THAT END TWO METHODS ARE DESCRIBED IN THIS THESIS. FINALLY AN OVERVIEW IS GIVEN OF THE CHALLENGES OCCURRING WHEN IMPLEMENTING THE DEVELOPED INKJET TECHNOLOGY AND CREATING A 3D LAYER-WISE PRODUCTION SYSTEM, OPENING THE PATH TOWARDS A THIRD INDUSTRIAL REVOLUTION. COMBINING MULTIPLE MATERIALS INTO ONE SINGLE PRODUCT GIVES NEW OPPORTUNITIES. THE DESCRIBED PROCESS IS USED IN ONE OF THE FIRST SYSTEMS CAPABLE OF GENERATING GRADIENTS IN MATERIAL PROPERTIES WITHIN ONE PRODUCT. UNTIL NOW ONLY NATURE WAS ABLE TO GENERATE THESE STRUCTURES. NOW PRODUCTION TECHNOLOGY IS STARTING TO CATCH UP. 